\title{
Genetic studies towards elucidation of drought tolerance of potato
}

\author{
Biructawit Bekele Tessema
}




\section{Thesis committee}

\section{Promotor}

Prof. Dr Richard G.F. Visser

Professor of Plant Breeding

Wageningen University \& Research

\section{Co-promotor}

Dr C. Gerard van der Linden

Group leader Abiotic Stress Research, Plant Breeding

Wageningen University \& Research

\section{Other members}

Prof. Dr Paul C. Struik, Wageningen University \& Research

Prof. Dr Mark G.M. Aarts, Wageningen University \& Research

Prof. Dr Anton J. Haverkort, Ömer Halisdemir University, Turkey

Dr J. Wilco Ligterink, Wageningen University \& Research

This research was conducted under the auspices of the Graduate School of Experimental Plant Sciences. 


\title{
Genetic studies towards elucidation of drought tolerance of potato
}

\author{
Biructawit Bekele Tessema
}

Thesis

submitted in fulfillment of the requirements for the degree of doctor

at Wageningen University

by the authority of the Rector Magnificus,

Prof. Dr A.P.J. Mol,

in the presence of the

Thesis Committee appointed by the Academic Board

to be defended in public

on Tuesday 13 June 2017

at 1:30 p.m. in the Aula. 
Biructawit Bekele Tessema

Genetic studies towards elucidation of drought tolerance of potato, 196 pages.

PhD thesis, Wageningen University, Wageningen, the Netherlands (2017) With references, with summary in English

ISBN: 978-94-6343-195-8

DOI: http://dx.doi.org/10.18174/413763 
for HIM 



\section{Contents}

Chapter 1 General introduction

Chapter 2 Understanding the genetic basis of potato development using a multi-trait QTL analysis

Chapter 3 Multiple QTLs contribute to tolerance to drought in potato grown under field conditions

Chapter 4 Unravelling the genetic components of drought tolerance of Potato grown under moderate water limitation

Chapter 5 Relationship between soil ground cover and tuber yield production in potato under drought stress conditions

Chapter 6 General discussion 153-183 Summary 185-186 Acknowledgments 189-190

About the author 191

Education certificate 193

Colophon 



\section{Chapter 1}

General introduction 



\section{Potato: origin and importance}

Potato (Solanum tuberosum L.) is a staple food with great economic value that ranks as the fourth most important food crop in the world. Globally potato is cultivated on 19 million hectare, being $8^{\text {th }}$ in terms of area under cultivation and with an estimated 325 million tons of annual production (Food and Agricultural Organization of the United Nations, 2012). Potato production provides food, employment and income as a cash crop (Scott et al. 2000). Potatoes have a high productivity per unit area with relatively little water consumption and take a short production time, thus being a candidate crop for food security.

The cultivated potato $S$. tuberosum is autotetraploid $(2 n=4 x=48)$. The domestication of potato dates back 6000 years in the central Andes, which is present-day southern Peru and northern Bolivia, when the native people started to select wild potato species for human use (Spooner et al. 2005). The modern cultivated potato (Solanum tuberosum) was domesticated from wild potato species of the Solanum brevicaule complex (Spooner et al. 2005). The genus Solanum has over 220 wild tuber bearing potato species and seven cultivated potato species (Hawkes and Jackson 1992). The variation in ploidy level is one of the most important features in potato taxonomy. The chromosome numbers in the wild species vary from diploid $(2 n=2 x=24)$, triploid $(2 n=2 x=36)$, tetraploid $(2 n=4 x=48)$, pentaploid $(2 n=5 x=60)$, to hexaploid $(2 n=6 x=72)$, while in cultivated potatoes this ranges from diploid to pentaploid. The majority of the diploid species are self-incompatible while tetraploids are self-compatible allopolyploids with disomic inheritance (Hawkes 1990). Wild and cultivated potato genetic resources provide a variety of reproductive and genetic features associated with species differentiation and breeding applications.

Cultivated potatoes can be classified as landraces or improved varieties. Landraces are native varieties still grown in South America today while improved varieties are grown around the world. Landrace potato cultivars are native to two areas in South America: the upland Andes from eastern Venezuela to northern Argentina and the lowlands of southcentral Chile (Ames and Spooner 2008). It was in the year 1557 that potato was first introduced to Europe (Ríos et al. 2007). The origin of the "European" potato is disputed with two competing hypotheses, one suggesting its origin from the Andes while another one suggests it to originate from lowland Chile. For the last 60 years it was accepted that European potato could have an Andean origin but recent studies suggest the European 
potatoes most likely came from both Andean and Chilean landraces (Ríos et al. 2007). By the 1700 s, potato cultivation was widespread in Europe and its worldwide cultivation began soon after (Hawkes and Francisco-Ortega 1993). The Irish potato famine caused by potato late blight disease, Phytophtera infestans, caused widespread famine and migration in Europe beginning in 1845. Late blight remains one of the most serious potato diseases worldwide, yet the potato crop persisted as a staple food throughout Europe.

Although there is no clear recored when potatoes was introduced to Africa, the first introduction of potato to Ethiopia was in 1858 by a German immigrant, Wilhelm Schimper (Kidane-Mariam 1980). However, the adoption of potato crop by the Ethiopian farmers occurred very gradually for several decades and its wider adoption occured only at the end of 19th century (Gebremedhin et al. 2001). As a non-cereal crop, potato is regarded as a secondary crop despite its potential as a food security crop. However, efforts are being made by different sectors including governmental research centerts and non governmental organization to increase the production potato in the suitable highland ares of the country.

\section{Potato production}

Potatoes are grown in about 125 countries with annual productions approaching to 325 million tons (FAO, 2012). Potatoes are consumed by more than a billion people worldwide on a daily basis. For a long period of time potatoes held a particular importance in temperate climates but this has changed in the last 20 years when the world potato production has undergone major changes. In the last few years, there has been a dramatic increase in potato production in the developing nations mainly due to an increase in productivity and area harvested (FAO 2013). At present, developing nations account for more than half of the global potato area and production (Haverkort and Struik 2015). Currently, the major potato producing countries are China, India, The Russia Federation, Ukraine and USA (FAO 2013).

In Africa, Ethiopia ranks at the 11th place in potato production with an estimated annual production of 525000 tons as of 2007 (FAO 2008). Ethiopia has the potential to be the highest potato producing country in Africa with widely available highland areas that best suit potato production. Potato can potentially be grown in $70 \%$ of arable land estimated to be 10Mha (FAO 2008; Hirpa et al. 2010).However, the current potato production in Ethiopia 
occupies small (0.16Mha) part of the available arable land (Hirpa et al. 2010). In Ethiopia, there are four major potato production areas that include the central, eastern, northwestern and southern parts of the country (Hirpa et al. 2010) and Figure 1. Collectively these areas accounts for the country's $83 \%$ of potato farmers, where $40 \%$ are located in north western of the country (CSA 2008/2009).

\section{Environmental factors affecting potato growth}

The potato plant is adapted to tropical highland cool temperatures and shorter photoperiods. In essence, the growth and development of potato is governed by many factors including temperature and photoperiod. Moreover, environmental stresses are limiting factors in potato production and productivity. Among the many abiotic stresses, drought is by far the most devastating abiotic stress affecting potato production worldwide.

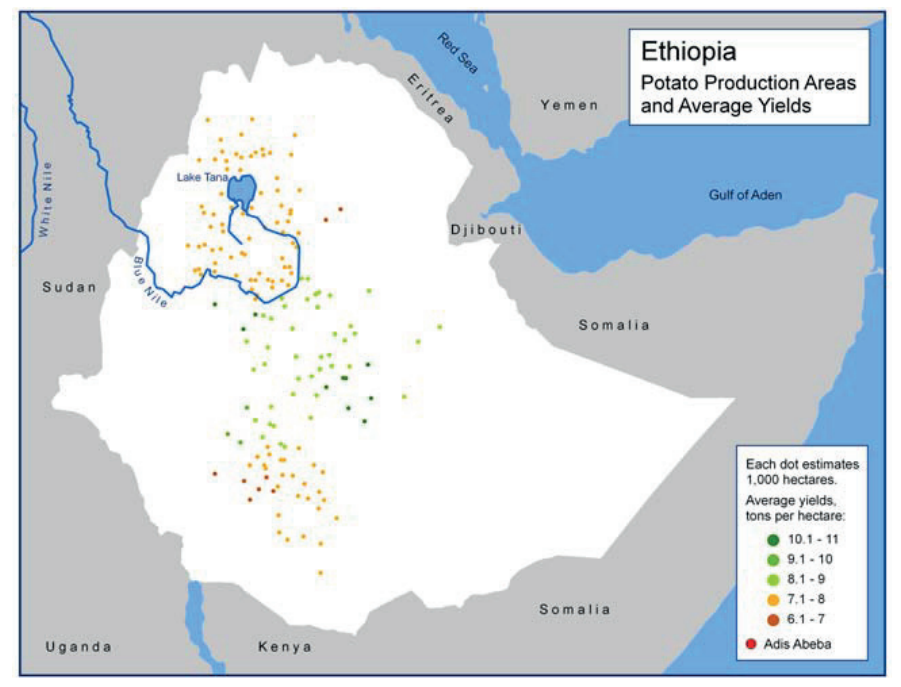

Figure 1. Potato production in Ethiopia along with the average yield in tons per hectare https://research.cip.cgiar.org/confluence/display/wpa/Ethiopia 


\section{Photoperiod and temperature}

The controlling effects of temperature and photoperiod on growth and tuberization of potato have been known and studied for many years (Ewing and Struik 1992; Levy and Veilleux 2007). Potatoes originate from cool tropical highlands with a daily temperature of $15-18^{\circ} \mathrm{C}$ and short photoperiods of $12 \mathrm{~h}$ (Ewing and Struik 1992) and most wild Solanum species are found in equatorial regions in South and Central America (Hijmans and Spooner 2001). Day lengths of 10 to $13 \mathrm{~h}$ are considered short days while long days have more than 14 hours of day light. Cultivated potatoes grown in temperate regions are believed to originate from Southern Chile and these produce tubers under long photoperiods (Ríos et al. 2007). The physiology of tuberization involves biochemical and molecular signals that link photoperiod perception in leaves to changes in cellular growth patterns in stolons (Sarkar 2010). The allelic variation that enables potato to tuberize under long day conditions has been elucidated (Kloosterman et al. 2013). Under short photoperiod, the potato plant tends to have less vegetative growth and to mature early (Van Dam et al. 1996). Time to tuber initiation is short under short days, which results in early maturation and senescence when coupled with higher temperature (Kooman et al. 1996). Under the long day and cool temperature of Northern Europe, the potato plant has the advantage of using 5-6 months of a growing season that allows longer period of photosynthesis, efficient translocation of assimilates to tubers and low transpiration rate to produce well, a situation that is beneficial for late maturing cultivars in particular.

The effect of temperature in potato is manifested through its effect on tuberization, where higher temperature delays tuber formation. Ideally potato is best suited to a cooler daily air temperature of 14 to $22^{\circ} \mathrm{C}$. The three developmental phases of potato: emergence to tuber initiation, tuber bulking, and maturation (senescence) are influenced by temperature and photoperiod (Kooman et al. 1996). Cooler temperatures (under $20^{\circ} \mathrm{C}$ ) along with short days promote tuber initiation and shorten the duration (Ewing and Struik 1992). For the second phase where dry matter is allocated to the tuber, the optimum temperature is between 14 and $22{ }^{\circ} \mathrm{C}$ (Ingram and McCloud 1984). At a temperatures above $23^{\circ} \mathrm{C}$ assimilates are allocated to the foliage at the cost of tuber growth (Haverkort and Harris 1987). Higher temperature (above $30^{\circ} \mathrm{C}$ ) under short photperiod induces crop senescence and promotes early maturity (Midmore 1984; Vander Zaag et al. 1990). 


\section{Drought}

As the change in environment pushes towards aridity, drought stress becomes one of the most recognized environmental constraints to date for plant survival and crop productivity (Dai 2011). The increasing aridity is a major factor threatening agriculture, as it is the major user of water resources in many regions of the world. The main reason for yield losses in global agriculture production is attributed to water shortage (Godfray et al. 2010). The impact of water scarcity in global agriculture production on food security is further enhanced by the growing number of people that needs to be fed. About $80 \%$ of cultivated land is based on rainfed agriculture and contributes to $60 \%$ of world food production (Rockström et al. 2003). As the resources such as water and land are further limited, food security in the twenty-first century will rely at least partly on development of improved cultivars with drought resistance and high yield stability (Pennisi 2008; Chapman et al. 2012). In order to achieve sound genetic improvement of crops for drought tolerance, a better understanding of the drought responses of plants is vital.

In Ethiopia major drought occurred following an El Nino resulting in decreased rainfall in the main rain season (June - September) but has increaseed rain in the small rainfall season (February - March) (Tsegay et al. 2001). In Ethiopia 85\% of the population is engaged in agriculture (CSA 2008/2009) and the dependency of most of the population on rain-fed agriculture makes food production highly vulnerable to the effects of the highly variable climate (Mersha and Boken 2005). The severity of drought stress varies in different parts of Ethiopia, where some part are highly affected by water shortage (Figure 2). Figure 2 shows deviation in soil moisture in the year 2015 from the average soil moisture of 1981 until 2014 for the main crop season (March to September). During the main cropping period soil moisture across Southern Afar, northern Somalia, eastern/central Oromia and eastern Amhara was the driest in at least 30 years. However, north east and southern part of the country shows normal or better soil moisture level. The changes in the soil moisture leve will have significant effect on crop yields andl indicate the importance of drought research that will help adapt crops to ever changing environmental conditions. 


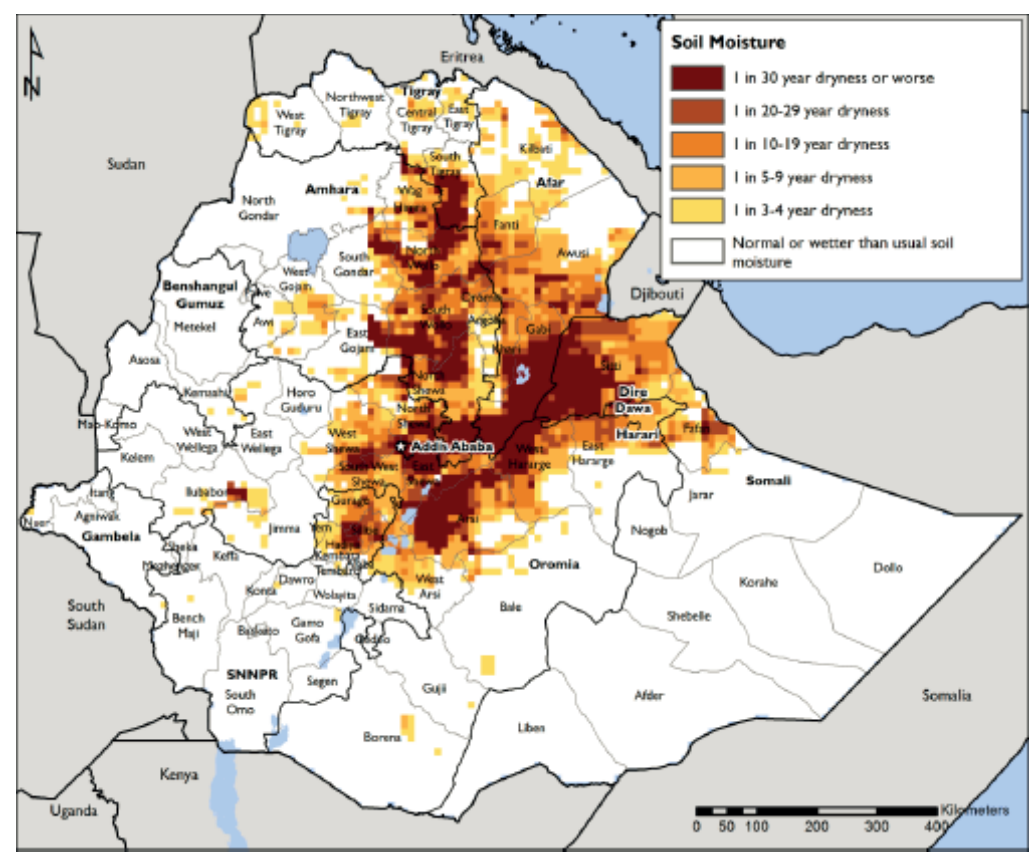

Figure 2 map showing deviation in soil moisture for the main cropping season of 2015 (March-September) versus the average soil moisture from 1981-2014in Ethiopia (source: FEWS NET)

\section{Drought response in plants}

Drought elicits complex responses in plants, initiating signal transduction pathway(s) that induce changes at the cellular, physiological, and morphological level Bray et al. (1993). Plant responses due to water limitation stress are classified as escape, avoidance, and tolerance. These three ways of responses are not mutually exclusive, as in practice we might observe combined responses.

\section{Escape}

Plants exhibit a high degree of developmental plasticity and are able to escape drought by completing their life cycle before physiological water deficit. Drought escape strategies rely on successful reproduction before the onset of severe stress and flowering time is an 
important trait Araus et al. (2002). A short life cycle is particularly advantageous in environments with terminal drought stress (Blum 1988; Araus et al. 2002). Breeding for short-duration varieties can help minimize yield loss due to drought stress that occurs at the latter developmental stages. However, yield is correlated with the length of crop duration and crops maturing early could result in reduction of the optimum yield Turner et al. (2001).

\section{Avoidance}

Dehydration avoidance in plants under drought stress conditions is achieved by keeping tissue water potential as high as possible through stomatal control of transpiration and by maintaining water uptake through an extensive root system (Turner et al. 2001). Dehydration avoidance mechanisms in plants are usually associated with adaptive morphophysiological traits (e.g., deep roots, early flowering, deposition of epicuticular waxes, osmotic adjustments, etc.). Water loss under stress conditions can be minimized by closing stomata or decreasing canopy leaf area through reduced growth and shedding of older leaves, while improvement in water uptake can be achieved through investing on root characteristics, such as increasing root depth and mass (Price et al. 2002). A deep and thick root system is helpful in extracting water from considerable depth.

Stomata closure and leaf growth inhibition are recognized as the earliest response for drought tolerance. This water saving strategy prevents cell dehydration and eventually cell death. However, drought-induced stomata closure reduces $\mathrm{CO} 2$ uptake by the leaves. The reduced inflow of $\mathrm{CO} 2$ into the leaves could spare more electrons for the formation of reactive oxygen species (Farooq et al. 2009). Reactive oxygen species (ROS) cause oxidative damage and impair the normal functions of cells (Foyer and Fletcher 2001). Moreover, the restriction of $\mathrm{CO} 2$ flow into the leaves results in a decline in photosynthesis (Chaves 1991). Stomata closure is mediated by chemical signals and the hormone Abscisic Acid (ABA), which was identified as one of the chemical signals involved in the regulation of stomatal functioning (Davies and Zhang 1991). ABA is synthesized in the shoot and root due to water limitation stress perceived by the plant. The accumulation of $A B A$ in response to drought stress may result from enhanced biosynthesis and/or a decrease in breakdown (Bray 1997)). It was further indicated that the accumulation of $A B A$ is correlated to the ability of roots to maintain growth under water stress conditions (Chaves et al. 2003). Drought stress signals 
mediated by ABA could results in the activation of drought responsive genes (Muijen et al. 2016).

\section{Tolerance}

Drought tolerance is defined as the relative capacity of a plant to maintain functional growth under low leaf water status (Chaves et al. 2003). Drought causes reduction in water potential of the cell, as a result of solute concentration gradients and osmosis, and leads to loss of cell turgor. Tolerance to low tissue water potential may involve osmotic adjustment, more rigid cell walls or smaller cells which will help in maintaining cell turgor (Obidiegwu et al. 2015). Osmotic balance is achieved through accumulation of compatible solutes or osmoprotectants called osmolytes and they can accumulate to high levels with out disrupting protein function (Bray 1997). Osmolytes synthesized in response to water stress may include amino acids (e.g. proline), sugar alcohols (e.g. pinitol), and quaternary ammonium compunds (e.g. glycine betaine) (Bray 1997). The enzyms involved in the synthesis of these compatible solutes allows an osmotic adjustment. Osmotic adjustment allows the cell to decrease osmotic potential and, as a consequence, increases the gradient for water influx and maintenance of turgor. The process of osmotic adjustment is crucial in plant adaptation to drought because it improves tissue water status which helps to maintain physiological activity during drought stress period and enables re-growth upon re-watering (Kramer and Boyer 1995). Other compounds that are induced during water stress include proteins such as dehydrins which belongs to late embryogenesis abundant (LEA) proteins group (Borovskii et al. 2002). Dehydrins may play an adaptive role in water related stresses. They have an important role in preserving the structural integrity of cells in vegetative plant tissues subjected to dehydration (Allagulova et al. 2003). Besides osmotic adjustment, reactive oxygen species (ROS) scavenging is reported to have an important role in protecting a plant from osmotic stress (Miller et al. 2010). ROS are toxic molecules that are capable of causing oxidative damage to protein, DNA, and lipids (Apel and Hirt 2004). During water stress there is higher accumulation of ROS and ROS scavenging enzymes such as superoxide dismutase, ascorbate peroxidase, catalase and peroxiredoxin act as ROS detoxifiers (Miller et al. 2010). 


\section{Drought response at the molecular level}

Drought response in plant is a complex process and better understanding of this complexity requires genomic tools such as expression analysis, metabolic profiling and proteomics. These analyses have been useful in understanding gene activation and regulation in response to drought stress. Stress related transcripts and proteins are categorized into two groups; functional and regulatory proteins Shinozaki and Yamaguchi-Shinozaki (1997). Functional proteins are involved in water stress response and cellular adaptation. Functional proteins include molecules such as chaperones, late embryogenesis abundant (LEA) proteins, osmotin, antifreeze proteins, mRNA-binding proteins, key enzymes for osmolyte biosynthesis (proline, betaine, sugars), water channel proteins, sugar and proline transporters, detoxification enzymes, and various proteases. Stress inducible genes encoding for such proteins have been used to improve stress tolerance in different transgenic crops. For instance, over-expressing barley group3 LEA gene HVA1 in rice and wheat was reported to improve osmotic stress tolerance and recovery after drought (Sivamani et al. 2000).

Regulatory proteins are involved in regulation of signal transduction and transcription in response to stress. These are transcription factors of multiple gene families such as dehydration-responsive element binding protein (DREB), ERF, Zinc finger, WRKY, MYB, MYC, HD-ZIP, bZIP, and NAC families. These transcriptional factors as well as components of signal transduction pathways coordinate expression of downstream regulons and have been used to engineer plants for stress tolerance. Genetically engineered crops with increased tolerance for stress using genes encoding the DREBs/CBFs transcription factors include tomato (Hsieh et al. 2002) and wheat (Pellegrineschi et al. 2004). An increase in drought tolerance by over-expressing the SNAC1 (Stress responsive NAC1) transcription factor in rice was reported (Hu et al. 2006).

\section{Drought effects on potato}

Potatoes are ideally suited for cooler growing conditions. Shortages of water from its optimum requirement can have significant effect on tuber yield production. The sensitivity of potatoes to water shortage is mainly due to its shallow and low density root system. The penetration of potato roots is only 0.5 to $1 \mathrm{~m}$ and about $85 \%$ of the roots are concentrated in 
the upper 0.3m of soil (Gregory and Simmonds 1992). These properties of potatoes make potato a poor conductor of water.

Several studies have shown the severe effects of drought stress on potato tuber yield (Deblonde and Ledent 2001; Anithakumari et al. 2012; Khan et al. 2015). The magnitude of drought effects on potato depends on the phenological timing, duration and severity of stress (Jefferies 1995). Water shortage during the early growth stages of potato affects final tuber yield and recovery is also difficult (Deblonde and Ledent 2001). The impact of water stress at the different growth stages of potato is illustrated in Figure 3.

The effects of water stress on morphological and physiological traits of potato have been studied by many researchers. Drought stress can decrease plant growth, leaf size, leaf number, shoot height and shortens growth cycle (Jefferies 1995; Deblonde and Ledent 2001). Drought stress also reduces ground coverage (Ojala et al. 1990). Water stress can have strong effects on physiological traits such as photosynthesis rate (Jefferies 1995). The effects of drought stress on morphological and physiological traits will result in limited tuber production (Anithakumari et al. 2012). This suggests that yield under water stress conditions is determined by the aggregated effects on morphological and physiological traits. The relative importance of each trait may depend on the severity of stress or plant growth stage. 

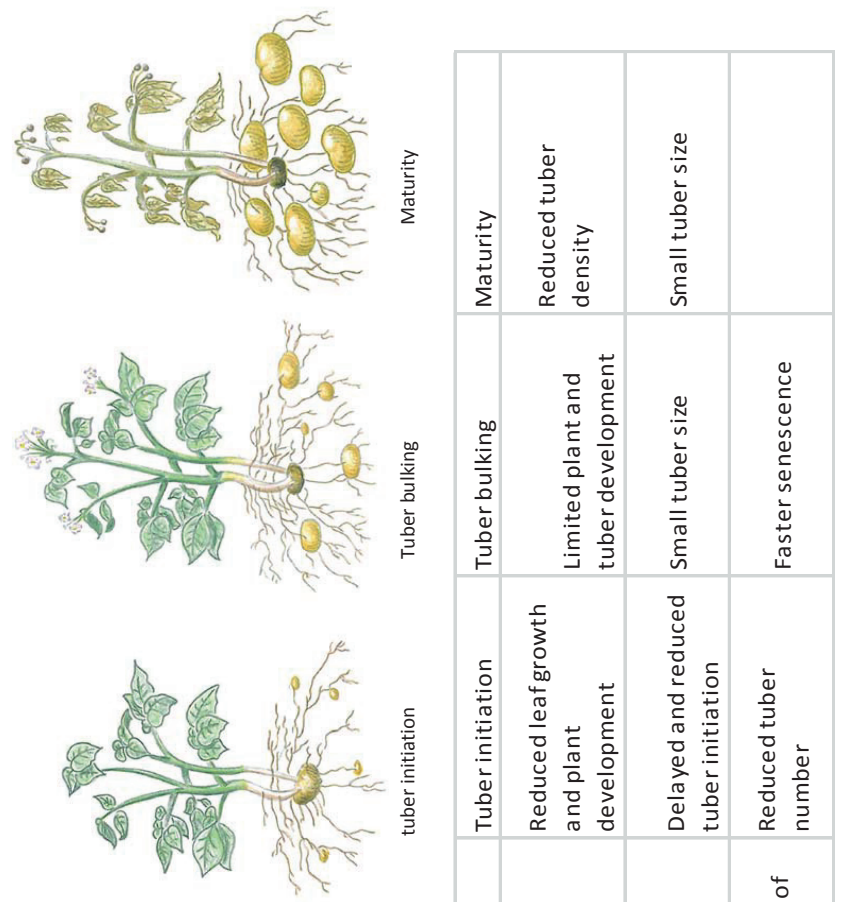

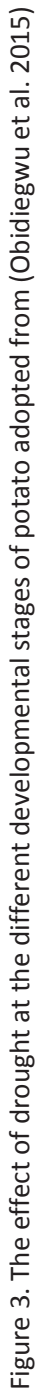




\section{Potato breeding for drought tolerance}

Drought is a major threat to agricultural production and drought tolerance is a prime target for molecular approaches to crop improvement. Drought is a complex polygenic trait and poses a challenge for drought tolerance breeding. Improving potato for drought tolerance at least requires the knowledge of physiological mechanisms and genetic control of the contributing traits at different plant developmental stages. Therefore identification of genetic variation for drought tolerance is the first step towards drought tolerance breeding. Compared to drought tolerance breeding for cereals, breeding for tolerance to drought in potato is in its early stages. Recently, studies in identification and understanding of the genetic basis of drought tolerance were done in diploid mapping populations (Anithakumari et al. 2011; Anithakumari et al. 2012; Khan et al. 2015). These studies have shown the presence of genetic variation for drought tolerance in potato and have outline the need for understanding agronomical, physiological, and morphological traits involved in drought responses and their interactions.

Wild potato species and adapted germplasm can serve as a great source of genetic variation for drought tolerance. Wild species of potatoes growing in its center of origin in SouthAmerica have adapted to harsh environments at high altitudes more than 3,000 meters above sea level and are regularly exposed to water-scarce conditions (Schafleitner et al. 2007). This genetic variation can further be exploited for the improvement of potato for drought tolerance. However, breeding for drought tolerance can be complicated by simultaneous occurrence of other abiotic (high temperature, salinity) and biotic stresses (diseases). Thus the success of breeding for increased drought tolerance depends on the integrated use of genomic approaches and precise phenotyping.

\section{Dissecting complex traits}

Most of the traits of interest in plant breeding such as yield or drought resistance are quantitative or complex traits. A quantitative trait does not only depend on the cumulative action of many genes but is also affected by the environment in which plants are growing and their interactions resulting in a continuous variation of phenotypes. The genetic variation of a quantitative trait is controlled by the collective effects of many genes called quantitative trait loci (QTL). A single phenotypic trait can be influenced by more than one 
QTL. Recent advances in genome mapping and genomics technologies have provided tools for molecular dissection of drought tolerance (Worch et al. 2011).

\section{QTL mapping}

The process of QTL mapping has been summarized in (Mir et al. 2012). The process involves the development of mapping populations segregating for stress tolerance-related traits, identification of polymorphic markers, genotyping of the mapping population with polymorphic markers, construction of genetic maps, phenotyping of traits, and QTL analysis using both genotypic and phenotypic data. QTL analysis have been useful in identification of the genetic basis of drought tolerance (Fleury et al. 2010). Several studies have used QTL mapping to genetically dissect drought tolerance in potato (Anithakumari et al. 2011; Anithakumari et al. 2012; Khan et al. 2015), wheat and barley (Fleury et al. 2010). These studies have been conducted under different environmental conditions including in vitro, greenhouse and field. Several QTLs were identified that controlled drought tolerance traits, including morphological, physiological and agronomical traits. These results suggest that tolerance in potato is determined by the combined effects of morphological and physiological traits. The results from these studies add to the fact that drought tolerance is a complex trait.

\section{Multi-trait QTL mapping}

Many studies have been done using QTL analysis to dissect the genetic basis of developmental traits in potato; However, the power of detecting QTLs linked to growth and developmental traits is higher when employing multi-trait QTL analysis compared to analyzing traits separately. The power of multi-trait QTL analysis lies in its ability to detect closely linked chromosomal regions affecting several traits simultaneously (Jiang and Zeng 1995). The first QTL meta-analysis in potato was done by projecting individual QTLS discovered for late blight and maturity from several studies on to a consensus map where it was possible to have consensus QTLs for the aforementioned traits simultaneously (Danan et al. 2011). This approach has allowed the improvement of defining the genomic regions controlling the traits. However, there are no reports made so far on the use of multi-trait analysis to understand the genetics that controls growth and developmental traits in potato. 


\section{Association mapping}

Association mapping is powerful approach for dissecting and understanding the genetic architecture of complex traits in crop species (Rafalski 2010). The principle of genome wide association mapping is to associate phenotypic variation with genetic markers in populations of unrelated genotypes by exploiting linkage disequilibrium (LD) between markers and QTLs (Malosetti et al. 2007; Ersoz et al. 2007). The advantages of association mapping over the linkage based QTL mapping is that it offers the possibility of exploiting all the recombination events that took place during the evolutionary history of a crop species resulting onto higher mapping resolution (Maccaferri et al. 2010). Successful application of association mapping for dissecting drought tolerance have been reported in barley (Varshney et al. 2012), maize (Xue et al. 2013) and wheat (Maccaferri et al. 2010). The feasibility of association mapping in tetraploid potato was represented in studies of (Simko 2004) and (Gebhardt et al. 2004). The usefulness of association mapping in potato was also shown by detecting marker- trait associations for quality traits in potato (D'hoop et al. 2008; D'Hoop et al. 2014). Recently, marker trait associations for physiological and agronomical traits in potato grown under high and low nitrogen inputs was reported (Ospina 2016). However, there are no reports in the use of association mapping to dissect drought tolerance in potato.

\section{Phenotyping}

The development of genomic approaches was very fast compared to the development of phenotypic technology in the past few decades. Molecular breeding is a general term used to describe modern breeding strategies where genotypic markers are used as a substitute for phenotypic selection (Ribaut et al. 2010). The development and use of molecular markers has accelerated breeding programs to produce improved cultivars through marker assisted breeding. However the importance of phenotyping in the genomics assisted breeding program was recently emphasized (Tuberosa 2012). Breeding experiments usually use large populations with many plants to be examined either in controlled (greenhouse) or open field environments, which makes phenotyping tedious and difficult. Recently, the development of high throughput phenotyping technology has made possible recording morphological and physiological traits. High throughput phenotyping platforms offers the possibility of detailed morphological and physiological measurements of plant characteristics that are non- 
destructive and invasive (Prasanna et al. 2013). Measuring traits such as canopy development, leave tissue water content, and photosynthetic status in plants has been possible though remote sensing phenotyping tool, image processing or infrared radiations.

Phenotyping can be even more challenging under drought stress conditions. The traits to be considered as potential selection targets for improving yield under water-limited conditions must be genetically correlated with yield and should have a greater heritability than yield itself (Blum 2011). Moreover, sufficient genetic variability of traits and lack of yield penalties under favourable conditions are also considered as desirable features (Tuberosa 2012). In measuring target trait under drought stress condition; non-destructive, rapid, accurate, and inexpensive measurements are recommended.

\section{Objectives and scope of this thesis}

In this thesis, we have performed drought stress trials to identify the genetic basis for drought tolerance in potato. We have conducted moderate drought stress experiments using a collection of potato cultivars under greenhouse conditions and severe drought stress experiments under field conditions in Ethiopia using the CXE diploid potato mapping population. We aimed to identify drought tolerance traits under moderate and severe drought stress conditions and elucidate the genetic basis controlling those traits.

In Chapter 2, the aim was to identify the genetic basis of plant developmental processes in potato by means of a multi-trait QTL analysis. For this analysis we have combined several traits describing plant development and agronomic characteristics measured under short day length of Ethiopia. The developmental traits (Plant height, flowering and senescence) were measured for several time points and were used for a curve fit. Parameters derived from fitted curves for flowering, senescence and plant height were simultaneously analysed with agronomic traits in a multi-trait QTL analysis to investigate the presence of pleiotropic genetic regions controlling those traits. We have identified pleiotropic QTLs influencing growth and agronomical traits and the relevance of multi-trait QTL analysis is also discussed.

In Chapter 3, the objective was to identify the genetic basis of morphological and physiological drought tolerance traits of potato grown under field conditions of Ethiopia. The 
CxE diploid potato mapping population was exposed to severe water stress and during the stress period data for several traits were collected. We performed QTL analysis on the collected trait data to find the genetic regions contributing to drought tolerance. We have identified 60 QTLs under well-watered and drought stress conditions. The implications of this result in breeding potato for improved drought tolerance are discussed.

In Chapter 4, with the aim to evaluate genetic diversity of moderate drought tolerance and identify genomic loci contributing to this drought tolerance in potato, we have evaluated a large set of potato cultivars for drought tolerance in the greenhouse. Several traits were collected and association mapping was performed to find significant marker trait associations both under well-watered and water -limited conditions. We were able to capture significant marker-trait associations under both treatment conditions. The implications of the marker-trait associations found under water limiting are discussed. The results of the genetic analyses under severe (chapter 3) and mild drought stress conditions are compared and discussed.

In Chapter 5, a subset of the CXE potato population was used to examine the effect of drought stress on the canopy development and its relation with tuber yield production. Time series data of canopy along with agronomic data were collected. Parameters extracted from the canopy curve were used to explain the ther relationship between canopy development and tuber yield under drought stress conditions. The relationship between these parameters and tuber yield production under water-limitined conditions is discussed.

In chapter 6, the results from drought stress experiments as well as the output from multitrait QTL analysis are further discussed. I also discuss the genetic basis of drought tolerance under mild and severe drought stress in more detail, as well as the implications for breeding potato for enhanced drought tolerance. I emphasize the importance of integrating different genomic approaches for a comprehensive understanding of the genetic basis of drought tolerance. 


\section{References}

Allagulova CR, Gimalov F, Shakirova F, Vakhitov V (2003) The plant dehydrins: structure and putative functions. Biochemistry (Moscow) 68 (9):945-951

Ames M, Spooner DM (2008) DNA from herbarium specimens settles a controversy about origins of the European potato. American Journal of Botany 95 (2):252-257

Anithakumari A, Dolstra O, Vosman B, Visser RG, van der Linden CG (2011) In vitro screening and QTL analysis for drought tolerance in diploid potato. Euphytica 181 (3):357-369

Anithakumari AM, Nataraja KN, Visser RG, van der Linden CG (2012) Genetic dissection of drought tolerance and recovery potential by quantitative trait locus mapping of a diploid potato population. Molecular breeding : new strategies in plant improvement 30 (3):1413-1429. doi:10.1007/s11032-012-9728-5

Apel K, Hirt H (2004) Reactive oxygen species: metabolism, oxidative stress, and signal transduction. Annu Rev Plant Biol 55:373-399

Araus J, Slafer G, Reynolds M, Royo C (2002) Plant breeding and drought in C3 cereals: what should we breed for? Annals of Botany 89 (7):925-940

Blum A (1988) Plant breeding for stress environments. CRC Press, Inc.,

Blum A (2011) Drought resistance-is it really a complex trait? Functional Plant Biology 38 (10):753-757

Borovskii GB, Stupnikova IV, Antipina AI, Vladimirova SV, Voinikov VK (2002) Accumulation of dehydrin-like proteins in the mitochondria of cereals in response to cold, freezing, drought and ABA treatment. Bmc Plant Biol 2 (1):1

Bray E, Moses M, Imai R, Cohen A, Plant A (1993) Regulation of gene expression by endogenous abscisic acid during drought stress. Current topics in plant physiology (USA)

Bray EA (1997) Plant responses to water deficit. Trends in plant science 2 (2):48-54

Chapman SC, Chakraborty S, Dreccer MF, Howden SM (2012) Plant adaptation to climate change-opportunities and priorities in breeding. Crop Pasture Sci 63 (3):251-268. doi:10.1071/cp11303

Chaves M (1991) Effects of water deficits on carbon assimilation. Journal of experimental botany $42(1): 1-16$

Chaves MM, Maroco JP, Pereira JS (2003) Understanding plant responses to drought-from genes to the whole plant. Functional plant biology 30 (3):239-264

CSA (2008/2009) Agricultural sample survey: Report on area and production of crops. Central Statistical Agency of Ethiopia (CSA)., Addis Ababa, Ethiopia

D'Hoop BB, Keizer PLC, Paulo MJ, Visser RGF, van Eeuwijk FA, van Eck HJ (2014) Identification of agronomically important QTL in tetraploid potato cultivars using a marker-trait association analysis. Theoretical and Applied Genetics 127 (3):731-748. doi:10.1007/s00122-013-2254-y

D'hoop BB, Paulo MJ, Mank RA, van Eck HJ, van Eeuwijk FA (2008) Association mapping of quality traits in potato (Solanum tuberosum L.). Euphytica 161 (1-2):47-60. doi:DOI 10.1007/s10681-007-9565-5

Dai A (2011) Drought under global warming: a review. Wiley Interdisciplinary Reviews: Climate Change 2 (1):45-65

Danan S, Veyrieras J-B, Lefebvre V (2011) Construction of a potato consensus map and QTL meta-analysis offer new insights into the genetic architecture of late blight resistance and plant maturity traits. Bmc Plant Biol 11 (1):1

Davies WJ, Zhang J (1991) Root signals and the regulation of growth and development of plants in drying soil. Annual review of plant biology 42 (1):55-76 
Deblonde P, Ledent J-F (2001) Effects of moderate drought conditions on green leaf number, stem height, leaf length and tuber yield of potato cultivars. European Journal of Agronomy 14 (1):31-41

Ersoz ES, Yu J, Buckler ES (2007) Applications of linkage disequilibrium and association mapping in crop plants. In: Genomics-assisted crop improvement. Springer, pp 97-119

Ewing E, Struik $P$ (1992) Tuber formation in potato: induction, initiation, and growth. Horticultural Reviews 14 (89):197

FAO (2008) Potato World: Africa-International Year of the Potato 2008. http://www.fao.org/potato-2008/en/world/africa.html. Accessed 07/12/ 2016

FAO (2013) FAOSTAT Database on Agriculture. FAO Food and Agriculture Organization of the United Nations. http://faostat.fao.org/. Accessed 20/09/ 2016

Farooq M, Wahid A, Kobayashi N, Fujita D, Basra S (2009) Plant drought stress: effects, mechanisms and management. In: Sustainable agriculture. Springer, pp 153-188

Fleury D, Jefferies S, Kuchel H, Langridge $P(2010)$ Genetic and genomic tools to improve drought tolerance in wheat. Journal of experimental botany 61 (12):3211-3222

Foyer C, Fletcher J (2001) Plant antioxidants: colour me healthy. Biologist (London, England) $48(3): 115$

Gebhardt C, Ballvora A, Walkemeier B, Oberhagemann P, Schuler K (2004) Assessing genetic potential in germplasm collections of crop plants by marker-trait association: a case study for potatoes with quantitative variation of resistance to late blight and maturity type. Mol Breeding 13 (1):93-102. doi:Doi 10.1023/B:Molb.0000012878.89855.Df

Gebremedhin G, Endale G, Kiflu B, Bekele K (2001) Country profile on potato production and utilization: Ethiopia. Ethiopian Agricultural Research Organization, Holetta Agricultural Research Center, National Potato Research Program.,

Godfray HCJ, Beddington JR, Crute IR, Haddad L, Lawrence D, Muir JF, Pretty J, Robinson S, Thomas SM, Toulmin C (2010) Food Security: The Challenge of Feeding 9 Billion People. Science 327 (5967):812-818. doi:10.1126/science.1185383

Gregory P, Simmonds L (1992) Water relations and growth of potatoes. In: The potato crop. Springer, pp 214-246

Haverkort A, Harris P (1987) A model for potato growth and yield under tropical highland conditions. Agricultural and forest meteorology 39 (4):271-282

Haverkort A, Struik P (2015) Yield levels of potato crops: Recent achievements and future prospects. Field Crops Research 182:76-85

Hawkes J, Jackson M (1992) Taxonomic and evolutionary implications of the Endosperm Balance Number hypothesis in potatoes. Theoretical and Applied Genetics 84 (1-2):180185

Hawkes JG (1990) The potato: evolution, biodiversity and genetic resources. Belhaven Press,

Hawkes JG, Francisco-Ortega J (1993) The early history of the potato in Europe. Euphytica 70 (1-2):1-7

Hijmans RJ, Spooner DM (2001) Geographic distribution of wild potato species. American Journal of Botany 88 (11):2101-2112

Hirpa A, Meuwissen MP, Tesfaye A, Lommen WJ, Lansink AO, Tsegaye A, Struik PC (2010) Analysis of seed potato systems in Ethiopia. American Journal of Potato Research 87 (6):537-552 
Hsieh T-H, Lee J-t, Charng Y-y, Chan M-T (2002) Tomato plants ectopically expressing Arabidopsis CBF1 show enhanced resistance to water deficit stress. Plant physiology 130 (2):618-626

Hu H, Dai M, Yao J, Xiao B, Li X, Zhang Q, Xiong L (2006) Overexpressing a NAM, ATAF, and CUC (NAC) transcription factor enhances drought resistance and salt tolerance in rice. Proceedings of the National Academy of Sciences 103 (35):12987-12992

Ingram K, McCloud D (1984) Simulation of potato crop growth and development. Crop Science 24 (1):21-27

Jefferies R (1995) Physiology of crop response to drought. In: Potato ecology and modelling of crops under conditions limiting growth. Springer, pp 61-74

Jiang C, Zeng Z-B (1995) Multiple trait analysis of genetic mapping for quantitative trait loci. Genetics 140 (3):1111-1127

Khan MA, Saravia D, Munive S, Lozano F, Farfan E, Eyzaguirre R, Bonierbale M (2015) Multiple QTLs linked to agro-morphological and physiological traits related to drought tolerance in potato. Plant Molecular Biology Reporter 33 (5):1286-1298

Kidane-Mariam HM (1980) Project proposal for the development of an Ethiopian potato program. Manuscript. Addis Ababa

Kloosterman B, Abelenda JA, Gomez MdMC, Oortwijn M, de Boer JM, Kowitwanich K, Horvath BM, van Eck HJ, Smaczniak C, Prat S, Visser RGF, Bachem CWB (2013) Naturally occurring allele diversity allows potato cultivation in northern latitudes. Nature 495 (7440):246-250

Kooman P, Fahem M, Tegera P, Haverkort A (1996) Effects of climate on different potato genotypes 2. Dry matter allocation and duration of the growth cycle. European Journal of Agronomy 5 (3):207-217

Kramer PJ, Boyer JS (1995) Water relations of plants and soils. Academic press,

Levy D, Veilleux RE (2007) Adaptation of potato to high temperatures and salinity-a review. American Journal of Potato Research 84 (6):487-506

Maccaferri M, Sanguineti MC, Demontis A, El-Ahmed A, del Moral LG, Maalouf F, Nachit M, Nserallah N, Ouabbou H, Rhouma S (2010) Association mapping in durum wheat grown across a broad range of water regimes. Journal of experimental botany:erq287

Malosetti M, van der Linden CG, Vosman B, van Eeuwijk FA (2007) A mixed-model approach to association mapping using pedigree information with an illustration of resistance to Phytophthora infestans in potato. Genetics 175 (2):879-889. doi:DOI 10.1534/genetics.105.054932

Mersha E, Boken VK (2005) Agricultural drought in Ethiopia. Oxford University Press,

Midmore D (1984) Potato (Solanum spp.) in the hot tropics I. Soil temperature effects on emergence, plant development and yield. Field Crops Research 8:255-271

Miller G, Suzuki N, CIFTCI-YILMAZ S, Mittler R (2010) Reactive oxygen species homeostasis and signalling during drought and salinity stresses. Plant, cell \& environment 33 (4):453467

Mir RR, Zaman-Allah M, Sreenivasulu N, Trethowan R, Varshney RK (2012) Integrated genomics, physiology and breeding approaches for improving drought tolerance in crops. Theoretical and Applied Genetics 125 (4):625-645. doi:DOI 10.1007/s00122-0121904-9

Muijen D, Anithakumari A, Maliepaard C, Visser RG, Linden CG (2016) Systems genetics reveals key genetic elements of drought induced gene regulation in diploid potato. Plant, Cell \& Environment 
Obidiegwu JE, Bryan GJ, Jones HG, Prashar A (2015) Coping with drought: stress and adaptive responses in potato and perspectives for improvement. Front Plant Sci 6 (542). doi:10.3389/fpls.2015.0052

Ojala J, Stark J, Kleinkopf G (1990) Influence of irrigation and nitrogen management on potato yield and quality. American Potato Journal 67 (1):29-43

Ospina CA (2016) Nitrogen use efficiency in potato: an integrated agronomic, physiological and genetic approach. PhD Thesis, Wageningen University, Wageningen,

Pellegrineschi A, Reynolds M, Pacheco M, Brito RM, Almeraya R, Yamaguchi-Shinozaki K, Hoisington D (2004) Stress-induced expression in wheat of the Arabidopsis thaliana DREB1A gene delays water stress symptoms under greenhouse conditions. Genome / National Research Council Canada $=$ Genome $/$ Conseil national de recherches Canada $47(3): 493-500$

Pennisi E (2008) Plant genetics: The blue revolution, drop by drop, gene by gene. Science 320 (5873):171-173. doi:10.1126/science.320.5873.171

Prasanna BM, Araus JL, Crossa J, Cairns JE, Palacios N, Das B, Magorokosho C (2013) HighThroughput and Precision Phenotyping for Cereal Breeding Programs. In: Gupta KP, Varshney KR (eds) Cereal Genomics II. Springer Netherlands, Dordrecht, pp 341-374. doi:10.1007/978-94-007-6401-9_13

Price AH, Cairns JE, Horton P, Jones HG, Griffiths H (2002) Linking drought-resistance mechanisms to drought avoidance in upland rice using a QTL approach: progress and new opportunities to integrate stomatal and mesophyll responses. Journal of experimental botany 53 (371):989-1004

Rafalski JA (2010) Association genetics in crop improvement. Current opinion in plant biology 13 (2):174-180. doi:DOI 10.1016/j.pbi.2009.12.004

Ribaut J, De Vicente M, Delannay X (2010) Molecular breeding in developing countries: challenges and perspectives. Current opinion in plant biology 13 (2):213-218

Ríos D, Ghislain M, Rodríguez F, Spooner DM (2007) What is the origin of the European potato? Evidence from Canary Island landraces. Crop Science 47 (3):1271-1280

Rockström J, Barron J, Fox P (2003) Water productivity in rain-fed agriculture: challenges and opportunities for smallholder farmers in drought-prone tropical agroecosystems. Water productivity in agriculture: Limits and opportunities for improvement 85199 (669):8

Sarkar D (2010) Photoperiodic inhibition of potato tuberization: an update. Plant growth regulation 62 (2):117-125

Schafleitner R, Gutierrez Rosales RO, Gaudin A, Alvarado Aliaga CA, Martinez GN, Tincopa Marca LR, Bolivar LA, Delgado FM, Simon R, Bonierbale M (2007) Capturing candidate drought tolerance traits in two native Andean potato clones by transcription profiling of field grown plants under water stress. Plant physiology and biochemistry : PPB / Societe francaise de physiologie vegetale 45 (9):673-690. doi:10.1016/j.plaphy.2007.06.003

Scott GJ, Rosegrant MW, Ringler C (2000) Global projections for root and tuber crops to the year 2020. Food policy 25 (5):561-597

Shinozaki K, Yamaguchi-Shinozaki K (1997) Gene expression and signal transduction in waterstress response. Plant physiology 115 (2):327

Simko I (2004) One potato, two potato: haplotype association mapping in autotetraploids. Trends in plant science 9 (9):441-448. doi:DOI 10.1016/j.tplants.2004.07.003

Sivamani E, Bahieldin A, Wraith JM, Al-Niemi T, Dyer WE, Ho T-HD, Qu R (2000) Improved biomass productivity and water use efficiency under water deficit conditions in 
transgenic wheat constitutively expressing the barley HVA1 gene. Plant Science 155 (1):1-9

Spooner D, Nunez J, Rodriguez F, Naik P, Ghislain M (2005) Nuclear and chloroplast DNA reassessment of the origin of Indian potato varieties and its implications for the origin of the early European potato. Theoretical and applied genetics 110 (6):1020-1026

Tsegay W, Demlew A, Yibrah H (2001) Ethiopia country case study: Impacts and response to the 1997-98 El Nino. In: Once Burned Twice Shy? Lessons learned from the 1997-98. El Nino

United Nations University Press, Tokyo

Tuberosa R (2012) Phenotyping for drought tolerance of crops in the genomics era. Frontiers in physiology 3:347. doi:10.3389/fphys.2012.00347

Turner NC, Wright GC, Siddique K (2001) Adaptation of grain legumes (pulses) to waterlimited environments. Advances in Agronomy 71:193-231

Van Dam J, Kooman P, Struik P (1996) Effects of temperature and photoperiod on early growth and final number of tubers in potato (Solanum tuberosum L.). Potato Research 39 (1):51-62

Vander Zaag P, Demagante A, Ewing E (1990) Influence of plant spacing on potato (Solanum tuberosum L.) morphology, growth and yield under two contrasting environments. Potato research 33 (3):313-323

Varshney R, Paulo M, Grando S, Van Eeuwijk F, Keizer L, Guo P, Ceccarelli S, Kilian A, Baum M, Graner A (2012) Genome wide association analyses for drought tolerance related traits in barley (Hordeum vulgare L.). Field Crops Research 126:171-180

Worch S, Rajesh K, Harshavardhan VT, Pietsch C, Korzun V, Kuntze L, Börner A, Wobus U, Röder MS, Sreenivasulu N (2011) Haplotyping, linkage mapping and expression analysis of barley genes regulated by terminal drought stress influencing seed quality. Bmc Plant Biol $11(1): 1$

Xue Y, Warburton ML, Sawkins M, Zhang X, Setter T, Xu Y, Grudloyma P, Gethi J, Ribaut J-M, Li W (2013) Genome-wide association analysis for nine agronomic traits in maize under well-watered and water-stressed conditions. Theoretical and applied genetics 126 (10):2587-2596 



\section{Chapter 2 \\ Understanding the genetic basis of potato development using a multi-trait QTL analysis}

P. Hurtado López ${ }^{1,2,5}$, Biructawit B.Tessema ${ }^{1,6}$, S.K. Schnabel ${ }^{2,3}$, C. Maliepaard ${ }^{1}$, C. Gerard Van der Linden ${ }^{1}$, P.H.C. Eilers ${ }^{2,4}$, J. Jansen ${ }^{2,3}$, F. van Eeuwijk ${ }^{2,3}$ \& Richard G.F. Visser ${ }^{1,3}$

${ }^{1}$ Plant Breeding, Wageningen University\& Research, Wageningen, the Netherlands

${ }^{2}$ Biometris, Wageningen University \&Research, Wageningen, the Netherlands

${ }^{3}$ Centre for BioSystems Genomics, Wageningen, the Netherlands

${ }^{4}$ Erasmus University Medical Center. Rotterdam, the Netherlands

${ }^{5}$ C.T. de Wit Graduate School for Production Ecology and Resource Conservation (PE\&RC).

Wageningen University, Wageningen, the Netherlands

${ }^{6}$ The graduate school of Experimental Plant Sciences (EPS), Wageningen University,

Wageningen, the Netherlands

Published in EUphytica (2015) 204 (1):229-241

Doi10.1007/s10681-015-1431-2 


\begin{abstract}
Understanding the genetic basis of plant development in potato requires a proper characterization of plant morphology over time. Parameters related to different aging stages can be used to describe the developmental processes. It is attractive to map these traits simultaneously in a QTL analysis; because the power to detect a QTL will often be improved and it will be easier to identify pleiotropic QTLs. We included complex, agronomic traits together with plant development parameters in a multi-trait QTL analysis. First, the results of our analysis led to coherent insight into the genetic architecture of complex traits in potato. Secondly, QTL for parameters related to plant development were identified. Thirdly, pleiotropic regions for various types of traits were identified. Emergence, number of main stems, number of tubers and yield were explained by 9, 5, 4 and 6 QTL, respectively. These traits were measured once during the growing season. The genetic control of flowering, senescence and plant height, which were measured at regular time intervals, was explained by 9,10 and 12 QTL, respectively. Genetic relationships between aboveground and belowground traits in potato were observed in 14 pleiotropic QTL. Some of our results suggest the presence of QTL-by-Environment interactions. Therefore, additional studies comparing development under different photoperiods are required to investigate the plasticity of the crop.
\end{abstract}

Keywords: Development, Multi-trait analysis, Plant development, Pleiotropy, Potato, Senescence 


\section{Introduction}

The development of plants is a complex, dynamic process controlled by networks of genes as well as environmental factors. As a consequence, QTL analysis of traits related to plant development requires the use of advanced statistical-genetic models and methods (Atchley 1984; Wolf et al. 2001). Conventional QTL mapping strategies neglect the fact that traits related to plant development are changing in time. For example, in potato plant height and tuber size change in time, and their development is influenced by changing environmental factors during the growth season. Therefore, such traits should be represented by functions of time and/or variables describing the major changes in environmental factors over time. This requires an approach that is able to detect genetic effects related to plant development.

In Arabidopsis, molecular markers have been associated with phenotypes observed at different development stages and the differences between these stages have been compared (Mauricio 2005). In the same model plant, simulated time series data have been used to infer growth curves in order to study the quantitative nature of plant development (Mündermann et al. 2005). A more general strategy to study the genetic architecture of complex, dynamic traits, so- called functional mapping, has been proposed to integrate the development of traits in time into QTL mapping (Lin and Wu 2006; Wu and Lin 2006; Wu et al. 2003). Dissecting the genetic basis of plant development requires an accurate description of developmental morphology. Such descriptions are often lacking and conclusions are drawn based on observations of fully grown plants (Kellogg 2004). This means that comparisons between developmental phases are often superficial. Therefore, a proper characterization of development over time is needed to describe each part of the process.

In potato, previous studies have incorporated well characterised time series data into growth models and QTL analysis. This approach allowed a genetic description of senescence in terms of parameters related to different aging stages (Hurtado et al. 2012; Malosetti et al. 2006). To our knowledge, studies embedding plant development in potato into a simultaneous QTL analysis with complex, agronomic traits have not been reported. Therefore, the genetic control of plant development is still poorly understood. 
Although many QTL studies considered multiple traits, usually those traits were analysed separately. An integrated analysis combining traits related to developmental processes simultaneously is required to get a better understanding of the genetic and environmental forces driving plant development. QTL analysis combining data from multiple traits related to plant development will not only increase the power of QTL detection, it will also improve the understanding of the genetic control of developmental processes. As a consequence, a multi-trait QTL analysis of a single population allows the detection of closely linked chromosomal regions affecting several traits simultaneously (Jiang and Zeng 1995). Although different methodologies have been proposed not only to map multiple trait simultaneously (Jiang and Zeng 1995; Knott and Haley 2000; Malosetti et al. 2008) but also to differentiate between close linkage and pleiotropy of coincident QTL (Jiang and Zeng 1995; Knott and Haley 2000; Lebreton et al. 1998; Liu et al. 2007), the identification of pleiotropic genes requires additional genomic information such as high density linkage maps and genome sequence information.

A first attempt to estimate the optimal set of consensus QTL for several traits simultaneously in potato was done through a QTL meta-analysis (Danan et al. 2011). It permitted the colocalization of late blight resistance and plant maturity traits by projecting individual QTL onto a consensus map. However, there are no reports of such integrative analysis for developmental traits in potato. So far, data on traits related to plant development in potato have not been integrated in a single study in order to get insight into the genetic architecture of crop development and the presence of putative pleiotropic QTL related to plant development.

The aim of this study was to identify the genetic basis of plant developmental processes in potato by means of a multi-trait QTL analysis combining several traits describing plant development in time. A total of 23 traits related to plant development and agronomic value were incorporated in the multi-trait QTL analysis. For this purpose, a diploid potato mapping population was evaluated under field conditions. Plant height, flowering and senescence were assessed on a weekly basis. The agronomic traits yield, number of main stems and number of tubers were measured at harvest. We were interested in the presence and genetic positions of putative pleiotropic regions associated with plant development and 
traits of agronomic value. Fourteen pleiotropic QTL were detected in our study, providing insights into the genetic architecture of developmental processes and the genetic relationship between above and below ground traits in potato. The anchoring of putative pleiotropic QTL to the annotated potato genome sequence (Consortium 2011) will provide target genes for marker assisted breeding and candidate gene approaches.

\section{Materials and methods}

\section{Plant materials}

Potato development was assessed in the diploid backcross population, hereafter referred to as CXE. It was obtained from a cross between clone C (US- W5337.3 (Hanneman and Peloquin 1967); a hybrid between Solanum phureja (PI225696) and a dihaploid S. tuberosum (US-W42)) and clone E (a hybrid between VH34211 (a S. vernei-S. tuberosum back- cross) and clone C). CXE was developed for research purposes (Jacobs et al. 1995) based on the genetic background of the parents. It is known for its segregation of agronomic and quality traits (Celis-Gamboa 2002; Kloosterman et al. 2010) S.tuberosum and S. phureja have different day length requirements for tuberization making CxE suitable for the study of developmental processes influenced by photoperiod and other environmental conditions. In total, 190 genotypes were used in the experiment: parents $C$ and $E, 169$ genotypes of $C x E$, a selected group of nine European cultivars ('Astarte', 'Bintje', 'Gloria', 'Granola', 'Karnico', 'Mondial', 'Premie 're', 'Saturna' and 'Desiree') and 10 Ethiopian cultivars ('Awash', 'Belete', 'Bulle', 'Gera', 'Gorebella', 'Guassa', ‘Gu- dene', 'Jalene', 'Shenkolla' and 'Zengena').

\section{Experimental setup}

The CxE population was planted in a light clay soil under rain fed conditions on July16 2010 at Holetta Agricultural Research Center, Ethiopia (9.070N, 38.030E in West Ethiopia at an altitude of $2400 \mathrm{~m}$ ). Planting was done by hand, with a spacing of $75 \mathrm{~cm}$ between rows and $30 \mathrm{~cm}$ within rows. Fertilizer (165 kg UREA and $196 \mathrm{~kg}$ diammonium phosphate per hectare) was applied during planting and a fungicide (RidomilGold) was sprayed against late blight. Ridging was carried out three times throughout the experiment and weeding was done by hand whenever necessary. The experiment was laid out in a randomized complete block design with three blocks, laid against the slope of the field. In each block, the two parents, the CXE genotypes and the European and Ethiopian varieties were randomized over 190 
plots, with 4 plants per plot. The observation period of the developmental traits was 5 months (between July and December 2010) and meteorological data were obtained during this period from the meteorological service present at the research station. The air temperature was recorded daily, every $3 \mathrm{~h}$, day and night. Over the whole observation period, the temperature fluctuated between 4 and $23^{\circ} \mathrm{C}$ between 6 am and $6 \mathrm{pm}$ and during the night between 2 and $20^{\circ} \mathrm{C}$. During the experiment the day length was $12 \mathrm{~h}$.

\section{Agronomic traits}

During the growing period, for each plant the development was assessed by measuring aboveground and belowground traits. Aboveground, the date of emergence and the number of main stems were assessed once, while plant height, flowering and senescence were measured over time at regular intervals. Below- ground, number of tubers and total tuber weight were assessed after the final harvest.

The evaluation of flowering and senescence was done using a scale from 0 to 7 and 1 to 7 respectively, as described in (Celis-Gamboa et al. 2003). Flowering was recorded 17 times with intervals of $2-6$ days at $38,40,42,45,47,49,52,54,56,59,61,63,66,68,70,74,80$, $83,87,89$ and 95 days after planting (DAP). Senescence was assessed 16 times with intervals of $3-7$ days at $(80,83,87,91,95,99,103,107,111,115,119,123,129$ and 136 DAP.

Plant height was measured using the longest stem of each plant as the distance from ground level to main apex. The assessment was done at nine occasions with intervals of 6 days (26, $32,38,44,50,56,62,68$ and 74 DAP). All plots were harvested at 138 DAP and the tubers of each plant were counted and weighed.

\section{Conversion of days after planting into thermal days}

Crop development is mainly affected by temperature and can be modified by other factors such as photoperiod (Hodges 1990). Previous potato studies have shown that warm conditions lead to an acceleration of vegetative and reproductive development (flowers, berries) (Benoit et al. 1986; Haun 1975; Struik and Ewing 1995), whereas cooler conditions facilitate tuber growth (Marinus and Bodlaender 1975). The effect of temperature on crop development rate is often described by using a thermal-time concept. Thus, various nonlinear models have been developed to describe the temperature response of developmental processes in plants (Gao et al. 1992; Johnson and Thornley 1985; Yin et al. 1995). In our 
study, fluctuations in temperature under field conditions were accounted for by estimating the daily contribution of temperature to plant development. Calendar days after planting were transformed into thermal days after planting (TAP) using the non-linear temperature effect beta-function described by Yin et al. (1995). Day length was incorporated into this function as a constant (Masle et al. 1989). This was done to anticipate on a later comparison of the performance of the CXE population under different day length conditions. The nonlinear relationship between temperature, photoperiod and rate of growth is described by

$$
g\left(T_{i}\right)=\left[\left(\frac{T_{c}-T_{i}}{T_{c}-T_{o}}\right)\left(\frac{T_{i}-T_{b}}{T_{o}-T_{b}}\right)^{\frac{T_{o}-T_{b}}{T_{o}-T_{o}}}\right]^{c_{l}} l_{i}
$$

In which the three cardinal temperatures for phenological development of potato (base: Tb, optimal: To and ceiling: Tc) and the temperature response curvature coefficient, ct, have been assigned the values $\mathrm{Tb}=5.5{ }^{\circ} \mathrm{C}$, To $=23.4{ }^{\circ} \mathrm{C}, \mathrm{Tc}=34.6{ }^{\circ} \mathrm{C}$ and $\mathrm{ct}=1.7$, respectively (Khan 2012; Khan et al. 2013). Ti is the average daily air temperature and li is the light period as a proportion of a day on day i after planting. The new thermal unit is then the cumulative beta- thermal days after planting combining, temperature, time and photoperiod (photobeta thermal time, PBTT). This scale was used as the $x$-axis to analyse the time series data of plant height, flowering and senescence. PBTT will allow a better comparability of the traits across years and locations than normal time.

\section{Curve fitting and characterization of the curves}

Curve fitting of plant height, flowering and senescence was done using PBTT units on the $x$ axis. For modelling flowering and senescence we used a methodology previously described to fit senescence data in potato (Hurtado et al. 2012). A smooth generalized linear model was used to estimate smooth curves for the development of flowering and senescence over time. The estimation was done using the $R$ software environment (CoreTeam 2011). A different approach was used to model plant height. In contrast to flowering and senescence, plant height was measured as a continuous variable (in $\mathrm{cm}$ ). Up to twelve observations per 
genotype were available per time point. We pooled the 12 observations per genotype in each time point and fitted a curve to the relationship between plant height and time. A smooth expectile curve was well suited for this purpose and the expectiles were estimated using least asymmetrically weighted squares (Schnabel and Eilers 2009). They were combined with P-splines to provide a flexible functional form (Schnabel et al. 2012). This modeling procedure resulted in a smooth frontier curve to describe the development of plant height over time. For the calculations we used the package "expectreg" in R (Sobotka et al. 2012).

Parameters describing the development process were estimated by fitting the development curves to data. These parameters facilitated the study of development as continuous processes in time by breaking down the complex traits into components related to the different developmental stages. The first and second derivatives of the fitted curves have been used to characterise senescence processes under long day length conditions (Hurtado et al. 2012). The parameters used to characterise senescence were also used in our study to describe plant height, flowering and senescence under short photoperiod (Figure 1). These parameters are onset of development, mean and maximum progression rates (average and maximum speed of the development process), inflection point or the turning point at which the process enters into the final phase, and end of development. We also considered additional traits describing growth and development, such as maximum and mean plant height, duration of flowering and maximum progression rate for onset of plant height (maximum speed of the process between emergence and the first observation of plant height). Note that the parameters have different units and their interpretation is different. For instance, small values of progression rate indicate slow flowering, senescence or plant height processes, mainly associated to late genotypes; while small values of inflection point, onset or end are related to early genotypes. 


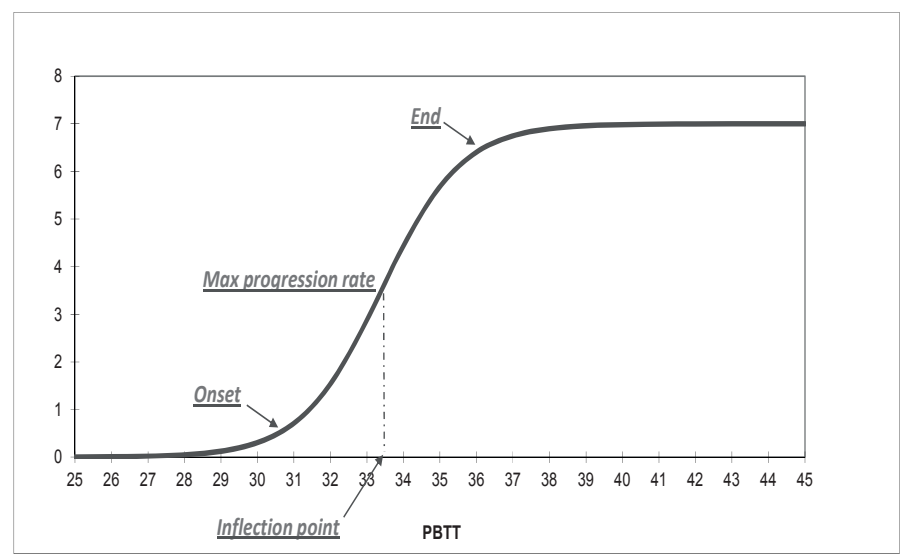

Figure 1. Fitted curve for flowering development of a random genotype of the CXE population. It is used as example to show the parameters describing flowering, senescence and plant height. On the $x$-axis: photo-beta thermal time (PBTT), on the $y$-axis: flowering on a scale from 0 to 7.

\section{Genetic maps and molecular data}

Single nucleotide polymorphism (SNP) markers scored in a core set of CxE (Anithakumari et al. 2010) were added to the maps of parents $C$ and $E$ as described in Hurtado et al. (2012). Together with the SNP markers, AFLP, SSR and CAPS with expected segregation ratios 1:1 and 1:1:1:1, respectively, were used to construct more saturated maps of parent $\mathrm{C}$ and $\mathrm{E}$ (Figure S1). JoinMap 4 (Van Ooijen 2009) was used to map 521 and 560 markers on the C and E maps, respectively, with 12 linkage groups (LG) for each parent as reported previously (Celis-Gamboa 2002).

Considering the differences in the recombination frequencies between the two parents (due to the fact that they originated from two different Solanum species), the $\mathrm{C}$ and $\mathrm{E}$ maps were not integrated. Markers segregating 1:1 and 1:1:1:1, were used in the QTL analysis; the latter ones were converted into two 1:1 types by separating the parental meioses in accordance with a pseudo-testcross analysis (Grattapaglia and Sederoff 1994). 
Table 1. Phenotypic traits included in the multi-trait QTL analysis, trait units and described developmental processes

\begin{tabular}{|c|c|c|c|}
\hline Trait type & Traits & Units & $\begin{array}{l}\text { Developmental } \\
\text { processes }\end{array}$ \\
\hline \multirow{6}{*}{$\begin{array}{l}\text { Parameters } \\
\text { derived from fitted } \\
\text { curves }\end{array}$} & Onset & Thermal days & $\begin{array}{l}\text { Flowering, senescence, } \\
\text { plant height }\end{array}$ \\
\hline & Maximum progression rate & & $\begin{array}{l}\text { Flowering, senescence, } \\
\text { plant height }\end{array}$ \\
\hline & Inflection point & Thermal days & $\begin{array}{l}\text { Flowering, senescence, plant } \\
\text { height }\end{array}$ \\
\hline & End & Thermal days & $\begin{array}{l}\text { Flowering, senescence, plant } \\
\text { height }\end{array}$ \\
\hline & Mean progression rate & & $\begin{array}{l}\text { Flowering, senescence, plant } \\
\text { height }\end{array}$ \\
\hline & $\begin{array}{l}\text { Maximum progression rate } \\
\text { in onset }\end{array}$ & & Plant height \\
\hline \multirow{7}{*}{$\begin{array}{l}\text { Characteristics } \\
\text { measured once } \\
\text { during the growing } \\
\text { season }\end{array}$} & Duration of flowering & Days after planting & Flowering \\
\hline & Maximum height & $\mathrm{cm}$ & Plant height \\
\hline & Mean height & $\mathrm{cm}$ & Plant height \\
\hline & Emergence & Days after planting & \\
\hline & Number of main stems & Number & \\
\hline & Total number of tubers & Number & \\
\hline & Yield & $\mathrm{Kg}$ & \\
\hline
\end{tabular}

\section{Multi-trait QTL analysis}

Two types of phenotypic traits were considered in our study (Table 1): growth and senescence curve parameters and agronomic plant characters measured on a single occasion during the growing season. For the agronomic traits, genotypic means were obtained from a linear model with blocks (three levels) and genotypes (169 levels). The curve parameters and the genotypic means for the agronomic traits were analysed together in a multi-trait QTL analysis (Alimi et al. 2013; Jiang and Zeng 1995; Stephens 2013), including 23 traits: five common traits for the three developmental processes (onset, maximum progression rate, inflection point, end and mean progression rate), one additional trait describing flowering (duration of flowering), three additional traits related to plant height (maximum progression of onset, maximum and mean height) and four agronomic traits (emergence, number of main stems, total number of tubers and yield). All the traits were standardized (subtracting the average and dividing by the standard deviation) to make traits with different scales and units comparable for the multi-trait analysis. 
For the multi-trait QTL analysis, the $\mathrm{C}$ and $\mathrm{E}$ maps were combined in a single map with linkage groups $\mathrm{C} 1, \ldots, \mathrm{C} 12$ and E1,..., E12. This allowed the use of markers of one parent as cofactors while searching for QTL in the other parent, thereby increasing the power to detect QTL. The QTL library of Genstat 15 (VSNi 2012) was used for the multi-trait QTL analysis by fitting the models as described by van Eeuwijk et al. (2010) and Alimi et al. (2013). The analysis started by fitting QTL models using simple interval mapping, SIM (Lander and Botstein 1989). The model that was fitted in SMI was; trait = trait intercept + trait specific QTL + residual genotypic effect + error. The residual genetic effects followed a multivariate normal distribution with an unstructured variance-covariance matrix.

The significance of trait-specific QTL was tested by a Wald test (Molenberghs and Verbeke 2000). A multiple testing correction was based on a Bonferroni procedure where effective number of tests is estimated from the genotype by marker score matrix as described in Li and Ji (2005), with a genome-wide test level of 0.05. A trait-specific confidence interval for QTL location was calculated according to Darvasi and Soller (1995). We adapted this procedure to the multi- trait context by choosing the shortest confidence interval among the individual traits following the original prescription to define the interval for all traits simultaneously (Alimi et al. 2013). We followed the strategy described by Boer et al. (2007) and Malosetti et al. (2014) to arrive at a final multi-QTL model; first a SIM scan was performed to identify a set of candidate QTL. The candidate QTL from the SIM scan was used as co-factors in a composite interval scan. After the composite interval scan, a backward elimination round was used to remove possibly redundant QTL. The percentage variance explained by a QTL was calculated as the square of the allelic substitution effect divided by the phenotypic variance based on trial means, multiplied by 100 (to obtain a percentage); this implicitly assumes a 1:1 segregation of the alleles at the QTL.

\section{Results}

\section{Curve fitting and characteristics of the curves}

Curves describing development over time were fitted to the data of the individuals of CXE, parents $\mathrm{C}$ and $\mathrm{E}$, and the control varieties. Differences in curve trajec- tories were observed between early and late genotypes for flowering, senescence and plant height (Figure 2). The maturity type of CxE was previously assessed under field conditions (Celis-Gamboa 2002) 
and it was used as reference in the present study. Early genotypes completed their life cycle faster and a complete S-shaped curve could be observed. Late genotypes showed slow progression of the developmental traits and some of them did not even complete the flowering and aging processes during the observation period. In that case, only the first part of the $\mathrm{S}$ shape could be observed.

In CXE a direct relationship was found between growth and maturity. Most of the late genotypes were tall and the early genotypes were short. However, the relationship between plant height and maturity did not hold for the Dutch cultivars (data not shown). For instance, Dutch varieties, irrespective of their matu- rity type, showed fast progression of senescence and all of them were shorter than the Ethiopian cultivars. This indicates that in these varieties maturation was accelerated whereas growth was restricted under short day conditions. In addition, flowering curves could not be fitted for the Dutch varieties due to the absence of flowering or flower abortion. Thus, the reduction in photoperiod affected the Dutch varieties dramatically; they are adapted to long day lengths. Suppressed flower development was also observed in previous potato studies in growth chambers where the irradiance was reduced (Clarke and Lombard 1939; Turner and Ewing 1988). In all CxE genotypes flowering and senescence curves presented parallel trajectories and they overlapped in early genotypes at the final stage of both processes. Examples are given in Figure 2.
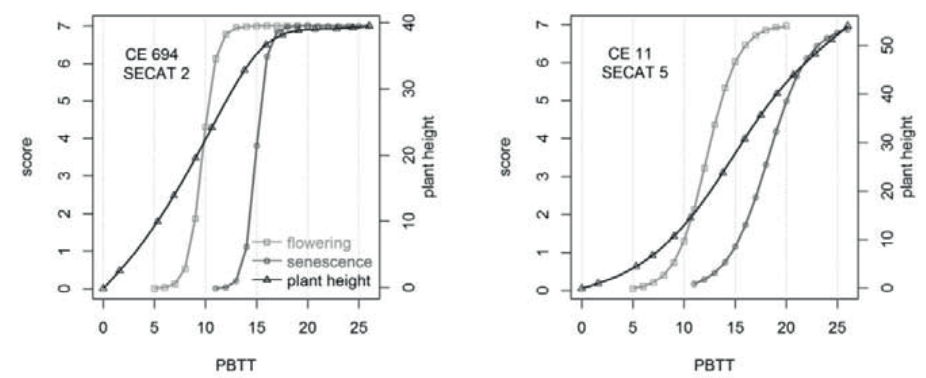

Figure 2. Fitted curves for plant height, flowering and senescence of two genotypes representing early and late maturing groups. On the x-axis: PBTT (Photo-beta thermal time) units combining average daily air temperature and photoperiod. On the $y$-axis: flowering and senescence scales from 0 to 7 (left) and plant height in $\mathrm{cm}$ on a continuous scale (right) 


\section{Genetics of complex traits}

The genetic architecture of complex developmental traits in potato was studied using the parameters derived from the fitted curves for flowering, senescence and plant height. Together with the agronomic traits they were included in a multi-trait QTL analysis and the QTL detected with the maternal and paternal maps could be observed in Figure 2. Although our study mainly focused on the presence and positions of QTL (upper plot of Figure 3) rather than on the allelic effects (lower plot), the QTL effects (positive: red; negative: blue) related to different values of the phenotypic traits, are also reported for the 23 traits on each QTL position. The size of QTL effects, indicated by the intensity of the colour (the darker the larger the effect), is also shown in Figure 2 and the explained variance for each trait is provided in Table 2. Opposite effects within pleiotropic regions are expected for a QTL related to negatively correlated traits. For instance, progression of flowering is negatively correlated to end of flowering (Additional file 2) and QTL effect on C5 and E5 were observed for both traits. Plants with fast flowering development (high values for progression rate) are expected to have an early end of the flowering process (small values for end of flowering).

\section{Complex traits}

For each complex, agronomic trait multiple QTL were identified (Figure 3). We checked the position of the QTL on the parental maps and the QTL detected on a particular linkage group were different from the QTL detected on the homologous linkage group in the other parent. Only one QTL was detected on C5 and E5 in the same genetic region. This was a major QTL associated with all developmental and agronomic traits (except emergence). In the E parent this QTL has a huge effect with values $-\log 10(p)$ going up to 50; for most traits, the explained variances for this QTL are very high going up to $60 \%$ for onset of senescence (Table 2). This finding is in agreement with previous reports indicating a major effect of a QTL in the same chromosomal region associated with plant maturity with pleiotropic effects on many developmental traits (Celis-Gamboa 2002; Hurtado et al. 2012; Kloosterman et al. 2013; Malosetti et al. 2006). According to our results there is no major contribution of this QTL to the agronomic traits as indicated by the low explained variances. Since our study focuses on new QTL (i.e. not the QTL on C5/E5 related to plant maturity) contributing to the understanding of the genetic architecture of complex traits, we have limited our discussion and main conclusions to those QTL. 
Flowering

In our study the genetic control of flowering was driven by 9 QTL. The QTL on C2, E1, E3 and E8 were associated with onset of flowering and other parameters of the flowering process (inflection point, maximum speed). The QTL on C10 and the first QTL on C5 with the total length of the flowering period and the end of flowering.

Senescence

In our study, ten QTL were found to be controlling the aging process. QTL on E1, E8 and E12 were related to onset of senescence and QTL on C3, C4 and E6 were associated with the end of senescence.

Plant height

We found 12 QTL related to plant height. QTL permanently expressed during the growing process were identified on C2, first half of C5, E5 and E12. QTL on C1, C3 and C4 were expressed between onset and half the growth process and they were also associated with the average and maximum plant height. The presence of common QTL for those traits could also be explained by the high phenotypic correlations between them (Additional file 2).

Agronomic traits

Emergence, number of main stems, total number of tubers and yield were explained by 9,5 , 4 and 6 QTL, respectively. These traits were measured once at the end of the growing season; therefore QTL related to the development of these traits could not be detected. Some QTL have been reported for yield on Chromosomes 1 and 6 in a tetraploid potato fullsib family (Bradshaw et al. 2008). In our study, QTL on C1 and E1 explained $11 \%$ of the phenotypic variance for yield suggesting the presence of a common genomic region on chromosome 1 in both parents for yield in potato. 

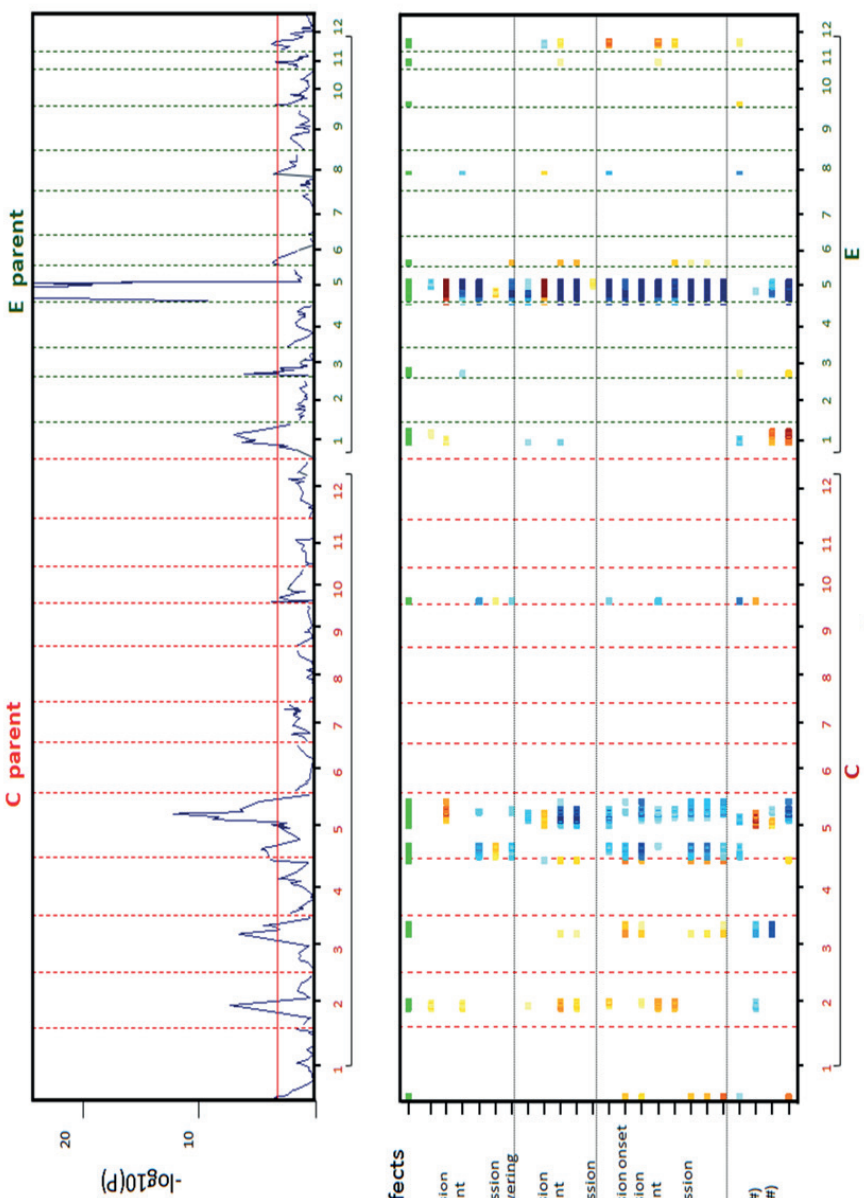

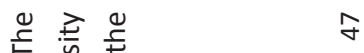

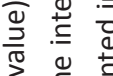

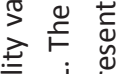

毒

을 는

ర 는 뉴

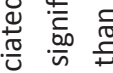

บू

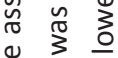

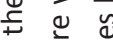

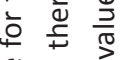

峁 迹

온 3 .

은 은 눈

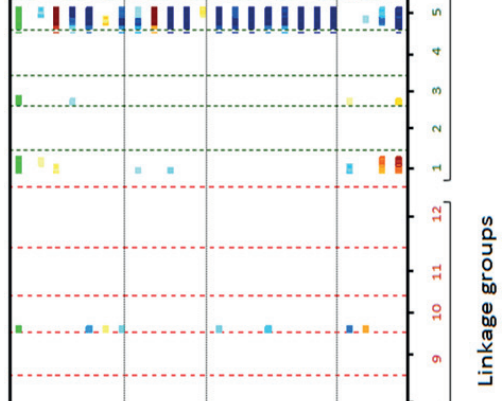

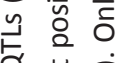

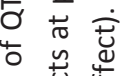

ญ 屯ै

苟

는 든 힘

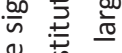

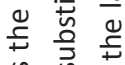

恣高㐫

능 잉

흐를 일

을 $\frac{N}{2}$

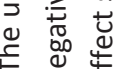

ᄃ $\stackrel{0}{\subseteq}$

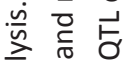

ते 항

$\triangle=$

范

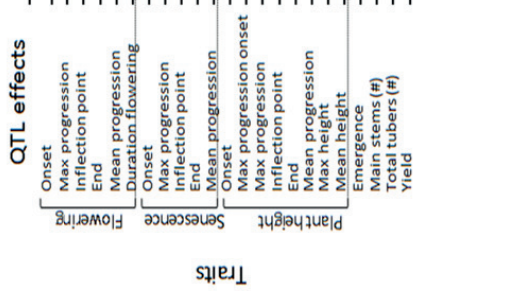

트응 응

응 은

뉸

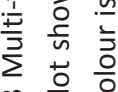

$m$ 응

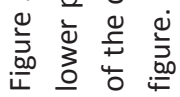


Although there was an effect of chromosome 5 on the agronomic traits, it was smaller compared with the effect on developmental traits, except for yield (Table 2). These results suggest that plant maturity does not play a central role in the agronomic traits considered in our study.

\section{Pleiotropic regions}

The multi-trait QTL analysis combining developmental and agronomic traits not only increased the power of QTL detection, compared with single trait linkage analysis (Table S2), but it also helped us to detect pleiotropic regions controlling aboveground and belowground traits in potato.

Fourteen pleiotropic QTL associated with developmental and agronomic traits could be identified in our study. In parent C, seven pleiotropic QTL were identified. For instance, the QTL on C2 was related with onset of plant height, flowering and senescence, progression of the three traits and number of main stems. The QTL on C3 was related to plant height, growth and number of tubers and number of main stems. In fact, previous studies have shown that tuber formation is reduced when the development of the haulm is accelerated (Maris 1964). A positive correlation between number of main stems and number of tubers has also been reported (Lemaga and Caesar 1990) but the genetic control of these traits is not yet clear. Here, we are able to report for both traits a QTL on C3 explaining 6 and $10 \%$ of the phenotypic variance for number of main stems and total number of tubers, respectively. The QTL on C10 was associated with emergence, onset of growth, duration of flowering and number of main stems per plant. This QTL could facilitate the selection of high yielding varieties with fast growth and a short flowering period. In the E parent, we detected one QTL on E10 associated with late emergence, seven pleiotropic QTL on E1, E3, E5, E6, E8, E11 and E12. For example, the QTL on E1 was associated with emergence, onset of senescence, number of tubers and yield, showing the highest explained variance for yield and number of tubers ( 8.1 and 6.9 \%, respectively). The QTL on E8 was associated emergence, onset of growth and senescence. The QTL on E12 is affecting the same traits. The QTL on E6 and E11 affected senescence and plant height, but had no effect on the agronomic traits. 
Further research will help to confirm the stability across environments of the pleiotropic regions associated with developmental traits found in our study and to investigate the presence of one or more genes in those regions. 


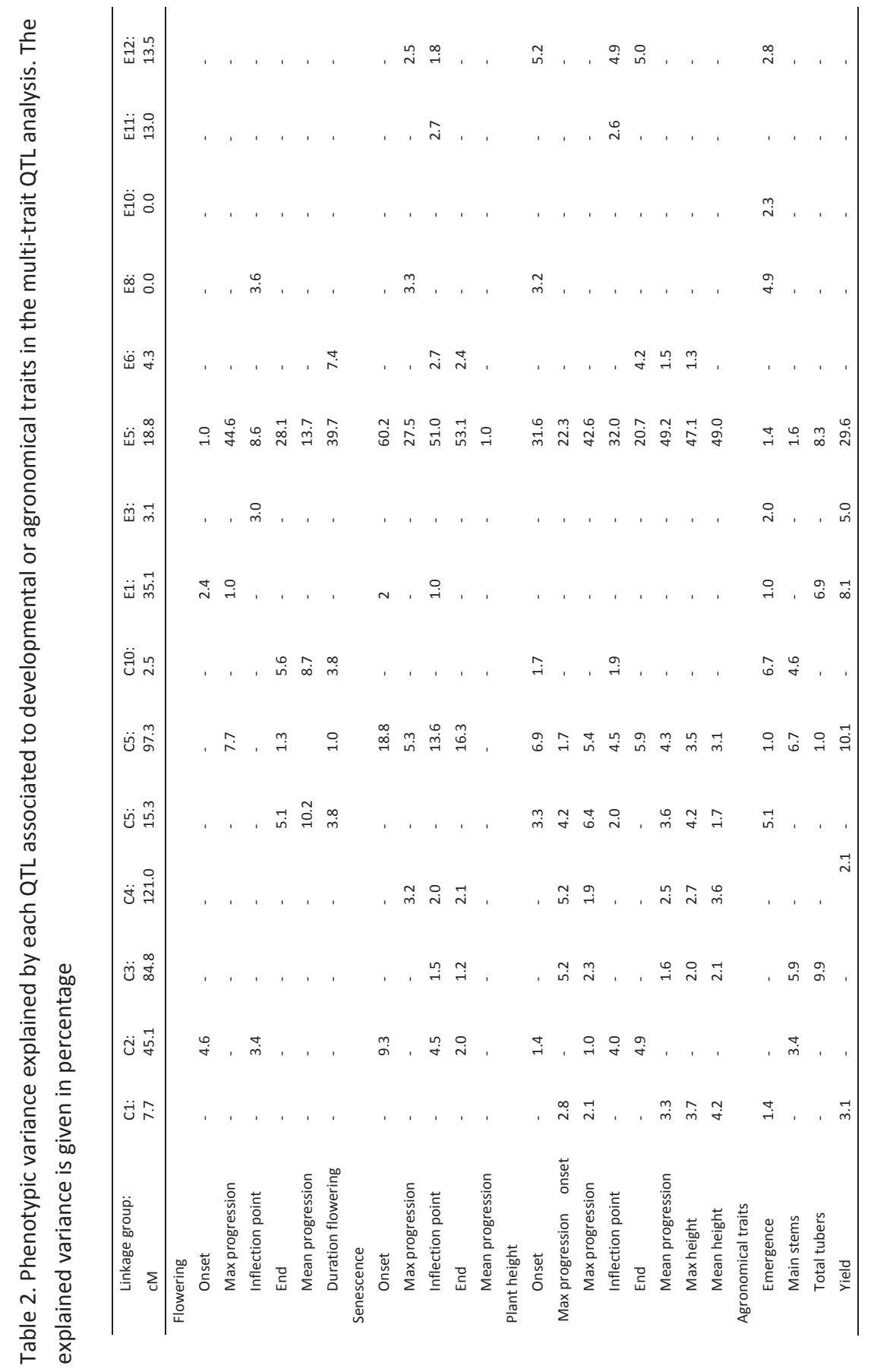




\section{Discussion}

The curve fitting approaches followed in our study provided an effective characterization of the developmental processes that occur during the potato life cycle under short day length conditions. The parameters derived from the curves characterise different stages of the development of the above ground parts of the plant. Plant height, flowering and senescence are described by five parameters: onset, end, progression rate (average and maximum speed of the process) and inflection point (time point when half of the developmental process has been reached) These parameters can also be used to characterise other processes in which growth curves are fitted using discrete or continuous data collected as a time series. For some traits additional characteristics were taken into account, such as duration of flowering or maximum plant height and they were directly calculated from the data. We also considered an additional trait for plant height (progression rate between emergence and the first observation of plant height) that was estimated from the fitted curves. It shows that the methodology we used for curve fitting permits not only the characterization of the processes with the conventional parameters, but also the estimation of new characteristics according to the needs of the study.

Differences in trajectories were observed when comparing the fitted developmental curves according to earliness. In the case of flowering and senescence, early genotypes showed a complete S-shaped curve whereas late genotypes show slow progression and only the first part of the S-shape was observed in most of the genotypes. As already known, the genomic region on chromosome 5 controlling maturity has a pleiotropic effect on developmental traits (Celis-Gamboa 2002; Malosetti et al. 2006; Hurtado et al. 2012) and it can explain the curve's trajectories defined according to earliness. On the other hand, there was no clear relation between plant height and maturity as was also observed in a previous study (Maris 1964). Photoperiod played a role in both development and agronomic performance of the plants. This was specially observed in the Dutch varieties used as controls in the experiment. They were shorter compared with their height in the Netherlands and all of them showed fast senescence development indicating that under short day length, growth was restricted and maturation was accelerated. Another indication of the photoperiod effect on development was the flower abortion of the Dutch varieties. It is known that reduction in day length can suppress flower development (Turner and Ewing 1988). 
To understand the genetic basis of the complex traits included in our study, developmental traits were treated as continuous and dynamic processes instead of looking at particular single moments of the life cycle. During the curve fitting all the time points were analysed together, a proper characterisation of different developmental stages was done and then the genetic factors underlying the processes were identified. A more efficient QTL analysis was performed using the estimated developmental parameters instead of searching for QTL per single time point. In addition, the number of QTL analyses was reduced. For instance, flowering was assessed in the field 17 times and we analysed only 6 parameters describing this trait. In the multi-trait QTL analysis presented here, all the parameters were analysed simultaneously and the presence of pleiotropic QTL was also investigated.

On the other hand, the combined use of parameters related to plant development and agronomic traits in a multi-trait QTL analysis provided coherent insight into (1) the genetic architecture of plant development and complex, agronomic traits in potato, (2) the presence of QTL for parameters related to plant development and (3) the genetic link between aboveground and belowground traits as discussed below.

For complex, agronomic traits, multiple QTL were identified explaining the genetic basis of these traits. Time-dependent QTL were detected for flowering, senescence and plant height. They showed a very low explained variance compared with the QTL expressed during the whole process (e.g. QTL related to mean progression rate). It has been reported that some QTL are expressed at early developmental stages and they are switched off after a particular age (Wu and Lin 2006). Time-dependent QTL have been observed in potato, controlling for instance onset and progression rate of senescence under long day length conditions (Hurtado et al. 2012).

We adapted the procedure of Darvasi and Soller (1995) to the multi-trait context by choosing the shortest confidence interval among the individual traits following the original prescription to define the interval for all traits simultaneously (Alimi et al. 2013). As one of the reviewers rightly mentioned, this may not be correct. Here we use it as a first approximation. We expect that our method is close to the true solution if for the trait concerned both the multi-trait analysis and the single-trait analysis put the QTL on the same 
position on the linkage map. Furthermore, our approximation will even be closer to the true solution if the sizes of the QTL effects in the single-trait analyses and the multi-trait analysis are approximately identical. For a general solution, how- ever, which should also involve the covariance structure of the traits, more research is needed.

Further research will help (1) to confirm the stability of the pleiotropic regions found in our study across environments, (2) to check the consistency of the allele effects, which can vary according to the environmental setup where they are expressed (Clark 2000) and (3) to investigate the presence of genes in regions where evidence of QTL exists. Some of our results suggest the presence of QTL 9 Environment interactions; additional studies comparing development under different photoperiods are required to take full advantage of the plasticity of the crop. Multi-environment experiments will allow us to better quantify the effect of the different photoperiod on traits, such as the ones presented in this study. The paper provides a detailed description of powerful, statistical-genetic methods that may also be useful to other crop species. It provides results on potato genetics that will further enhance potato breeding.

\section{Acknowledgements}

We would like to acknowledge Holetta agriculture research institute for experimental land provision and technical support. We thank Berend-Jan Domba and Melaku for their help in planting and phenotyping.

\section{References}

Alimi N, Bink M, Dieleman J, Magán J, Wubs A, Palloix A, Van Eeuwijk F (2013) Multi-trait and multi-environment QTL analyses of yield and a set of physiological traits in pepper. Theoretical and applied genetics 126 (10):2597-2625

Anithakumari A, Tang J, van Eck HJ, Visser RG, Leunissen JA, Vosman B, van der Linden CG (2010) A pipeline for high throughput detection and mapping of SNPs from EST databases. Mol Breeding 26 (1):65-75

Atchley WR (1984) Ontogeny, timing of development, and genetic variance-covariances structure. The American Naturalist 123 (4):519-540

Benoit G, Grant W, Devine O (1986) Potato top growth as influenced by day-night temperature differences. Agronomy Journal 78 (2):264-269 
Boer MP, Wright D, Feng L, Podlich DW, Luo L, Cooper M, van Eeuwijk FA (2007) A mixedmodel quantitative trait loci (QTL) analysis for multiple-environment trial data using environmental covariables for QTL-by-environment interactions, with an example in maize. Genetics 177 (3):1801-1813

Bradshaw JE, Hackett CA, Pande B, Waugh R, Bryan GJ (2008) QTL mapping of yield, agronomic and quality traits in tetraploid potato (Solanum tuberosum subsp. tuberosum). Theoretical and Applied Genetics 116 (2):193-211

Celis-Gamboa BC (2002) The life cycle of the potato (Solanum tuberosum L.): from crop physiology to genetics. PhD Thesis, Wageningen University, Wageningen. ,

Celis-Gamboa C, Struik P, Jacobsen E, Visser R (2003) Temporal dynamics of tuber formation and related processes in a crossing population of potato (Solanum tuberosum). Ann Appl Biol 143 (2):175-186

Clark M (2000) Comparative Genomics. 1st edn . Springer, Berlin

Clarke A, Lombard P (1939) Relation of length of day to flower and seed production in potato varieties. American Journal of Potato Research 16 (9):236-244

Consortium TPGS (2011) Genome sequence and analysis of the tuber crop potato. Nature 475:189-195

CoreTeam (2011) R: A language and environment for statistical computing. R Foundation for Statistical Computing, Vienna

Danan S, Veyrieras J-B, Lefebvre V (2011) Construction of a potato consensus map and QTL meta-analysis offer new insights into the genetic architecture of late blight resistance and plant maturity traits. Bmc Plant Biol 11 (1):1

Darvasi A, Soller M (1995) Advanced intercross lines, an experimental population for fine genetic mapping. Genetics 141 (3):1199-1207

Gao L, Jin Z, Huang Y, Zhang L (1992) Rice clock model-a computer model to simulate rice development. Agricultural and Forest Meteorology 60 (1-2):1-16

Grattapaglia D, Sederoff R (1994) Genetic linkage maps of Eucalyptus grandis and Eucalyptus urophylla using a pseudo-testcross: mapping strategy and RAPD markers. Genetics 137 (4):1121-1137

Hanneman R, Peloquin S (1967) Croosability of 24-chromosome potato hybrids with 48chromosome cultivars. European Potato Journal 10 (1):62-73

Haun J (1975) Potato growth-environment relationships. Agricultural Meteorology 15 (3):325-332

Hodges T (1990) Predicting crop phenology. Tyler \& Francis, Boca Ranton, FL, PP 7-13,

Hurtado PX, Schnabel SK, Zaban A, Veteläinen M, Virtanen E, Eilers PH, Van Eeuwijk FA, Visser RG, Maliepaard C (2012) Dynamics of senescence-related QTLs in potato. Euphytica 183 (3):289-302

Jacobs J, Van Eck H, Arens P, Verkerk-Bakker B, te Lintel Hekkert B, Bastiaanssen H, ElKharbotly A, Pereira A, Jacobsen E, Stiekema W (1995) A genetic map of potato (Solanum tuberosum) integrating molecular markers, including transposons, and classical markers. Theoretical and Applied Genetics 91 (2):289-300

Jiang C, Zeng Z-B (1995) Multiple trait analysis of genetic mapping for quantitative trait loci. Genetics 140 (3):1111-1127

Johnson I, Thornley J (1985) Temperature dependence of plant and crop process. Annals of Botany $55(1): 1-24$

Kellogg EA (2004) Evolution of developmental traits. Current opinion in plant biology 7 (1):92-98 
Khan MS (2012) Assessing genetic variation in growth and development of potato. PhD Thesis, Wageningen University, Wageningen

Khan MS, van Eck HJ, Struik PC (2013) Model-based evaluation of maturity type of potato using a diverse set of standard cultivars and a segregating diploid population. Potato Research 56 (2):127-146

Kloosterman B, Abelenda JA, Gomez MdMC, Oortwijn M, de Boer JM, Kowitwanich K, Horvath BM, van Eck HJ, Smaczniak C, Prat S, Visser RGF, Bachem CWB (2013) Naturally occurring allele diversity allows potato cultivation in northern latitudes. Nature 495 (7440):246-250

Kloosterman B, Oortwijn M, Uitdewilligen J, America T, de Vos R, Visser RGF, Bachem CWB (2010) From QTL to candidate gene: Genetical genomics of simple and complex traits in potato using a pooling strategy. Bmc Genomics 11. doi:Artn 158 Doi 10.1186/14712164-11-158

Knott SA, Haley CS (2000) Multitrait least squares for quantitative trait loci detection. Genetics 156 (2):899-911

Lander ES, Botstein D (1989) Mapping mendelian factors underlying quantitative traits using RFLP linkage maps. Genetics 121 (1):185-199

Lebreton CM, Visscher PM, Haley CS, Semikhodskii A, Quarrie SA (1998) A nonparametric bootstrap method for testing close linkage vs. pleiotropy of coincident quantitative trait loci. Genetics 150 (2):931-943

Lemaga B, Caesar K (1990) Relationships between numbers of main stems and yield components of potato (Solanum tuberosum L. cv. Erntestolz) as influenced by different daylengths. Potato Research 33 (2):257-267

Li J, Ji L (2005) Adjusting multiple testing in multilocus analyses using the eigenvalues of a correlation matrix. Heredity 95 (3):221-227

Lin M, Wu R (2006) A joint model for nonparametric functional mapping of longitudinal trajectory and time-to-event. Bmc Bioinformatics 7 (1):138

Liu J, Liu Y, Liu X, Deng H-W (2007) Bayesian mapping of quantitative trait loci for multiple complex traits with the use of variance components. The American Journal of Human Genetics 81 (2):304-320

Malosetti M, Ribaut J-M, van Eeuwijk FA (2014) The statistical analysis of multi-environment data: modeling genotype-by-environment interaction and its genetic basis. Drought phenotyping in crops: From theory to practice 4 (44):53

Malosetti M, Ribaut JM, Vargas M, Crossa J, Van Eeuwijk FA (2008) A multi-trait multienvironment QTL mixed model with an application to drought and nitrogen stress trials in maize (Zea mays L.). Euphytica 161 (1-2):241-257

Malosetti M, Visser R, Celis-Gamboa C, Van Eeuwijk F (2006) QTL methodology for response curves on the basis of non-linear mixed models, with an illustration to senescence in potato. Theoretical and applied genetics 113 (2):288-300

Marinus J, Bodlaender K (1975) Response of some potato varieties to temperature. Potato Research 18 (2):189-204

Maris B (1964) Studies concerning the relationship between plant height of potatoes in the seedling year and maturity in the clonal generations. Euphytica 13 (2):130-138

Masle J, Doussinault G, Farquhar G, Sun B (1989) Foliar stage in wheat correlates better to photothermal time than to thermal time. Plant, Cell \& Environment 12 (3):235-247

Mauricio R (2005) Ontogenesis of QTL: the genetic architecture of trichome density over time in Arabidopsis thaliana. Genetica 123:75-85 
Molenberghs G, Verbeke G (2000) Linear mixed models for longitudinal data. Springer,

Mündermann L, Erasmus Y, Lane B, Coen E, Prusinkiewicz P (2005) Quantitative modeling of Arabidopsis development. Plant physiology 139 (2):960-968

Schnabel S, Hurtado P, Eilers P, Maliepaard C, Van Eeuwijk F (2012) Statistical tools for preprocessing phenotypic data. In progress,

Schnabel SK, Eilers PH (2009) Optimal expectile smoothing. Computational Statistics \& Data Analysis 53 (12):4168-4177

Sobotka F, Schnabel SK, Schulze Waltrup L (2012) Expectreg: expectile and quantile regression $R$ Package, 0.35 edn edn.,

Stephens M (2013) A unified framework for association analysis with multiple related phenotypes. Plos One 8 (7):e65245

Struik P, Ewing E (1995) Crop physiology of potato (Solanum tuberosum): responses to photoperiod and temperature relevant to crop modelling. In: Potato ecology and modelling of crops under conditions limiting growth. Springer, pp 19-40

Turner A, Ewing E (1988) Effects of photoperiod, night temperature, and irradiance on flower production in the potato. Potato research 31 (2):257-268

van Eeuwijk FA, Bink MC, Chenu K, Chapman SC (2010) Detection and use of QTL for complex traits in multiple environments. Current opinion in plant biology 13 (2):193-205

Van Ooijen J (2009) MapQTL 6. Software for the mapping of quantitative trait loci in experimental populations of diploid species Kyazma BV: Wageningen, Netherlands

VSNi (2012) GenStat reference manual. VSN international, Hemel Hempstead 15th edn

Wolf JB, Frankino WA, Agrawal AF, Brodie III ED, Moore AJ (2001) Developmental interactions and the constituents of quantitative variation. Evolution 55 (2):232-245

Wu R, Lin M (2006) Functional mapping-how to map and study the genetic architecture of dynamic complex traits. Nat Rev Genet 7 (3):229-237

Wu R, Ma C-X, Zhao W, Casella G (2003) Functional mapping for quantitative trait loci governing growth rates: a parametric model. Physiological Genomics 14 (3):241-249

Yin X, Kropff MJ, McLaren G, Visperas RM (1995) A nonlinear model for crop development as a function of temperature. Agricultural and Forest Meteorology 77 (1):1-16 


\section{Supplementary files}

Figure S1. C and E linkage maps. The C map consists of 399 markers spanning 1403.3 cM with an average distance between adjacent markers of $3.5 \mathrm{cM}$. Two of the 12 LG (C10 and C12) were split in two sub-groups due to the large distance between adjacent markers (more than 30cM). The E map consists of 424 markers spanning $995.1 \mathrm{cM}$ with average distance between adjacent markers of $2.3 \mathrm{cM}$. Five of the $12 \mathrm{LG}(\mathrm{E} 1, \mathrm{E} 3, \mathrm{E} 6, \mathrm{E} 8, \mathrm{E} 11)$ were split in two subgroups due to the large distance between adjacent markers. The assignment of linkage groups was done according to (Celis-Gamboa 2002) and each LG is preceded by the letter C or $\mathrm{E}$ according to the parental map. 

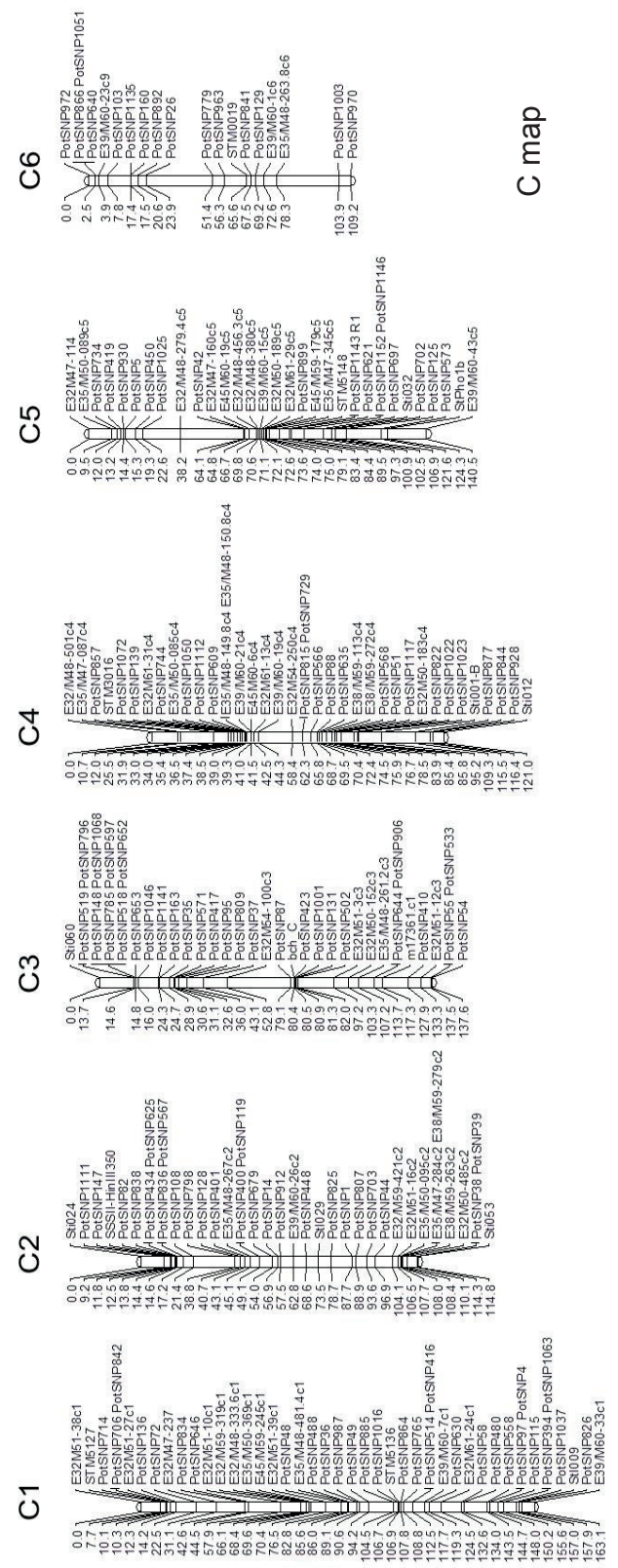
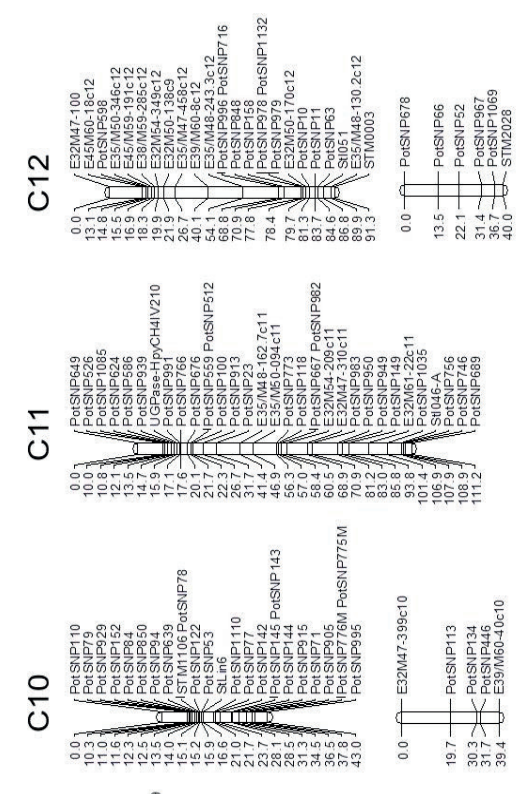

৩

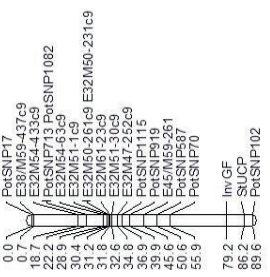

o
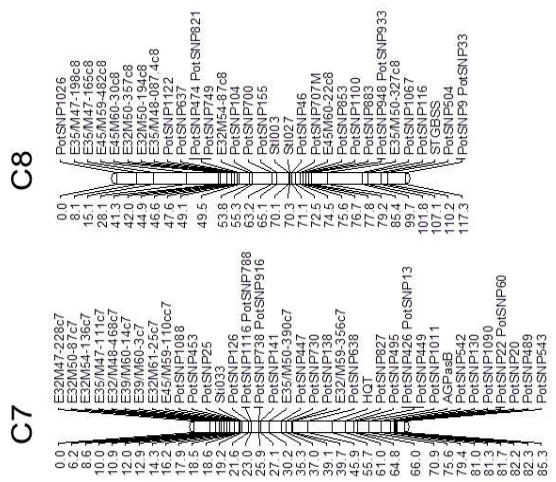
Ш

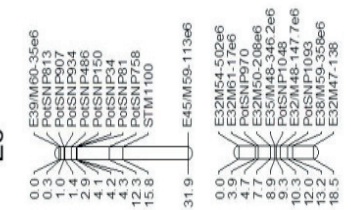

岃

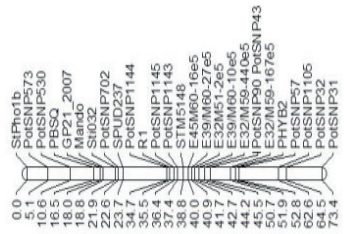

岌

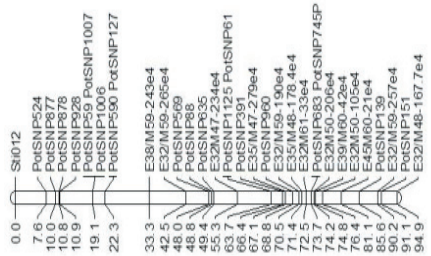

జึ
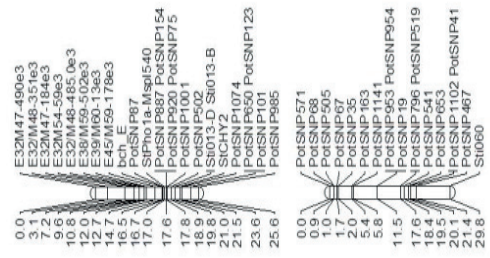

พี

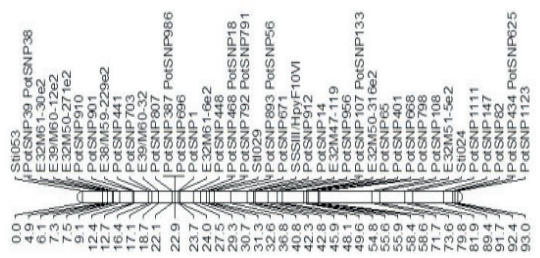

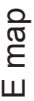

ㄴ
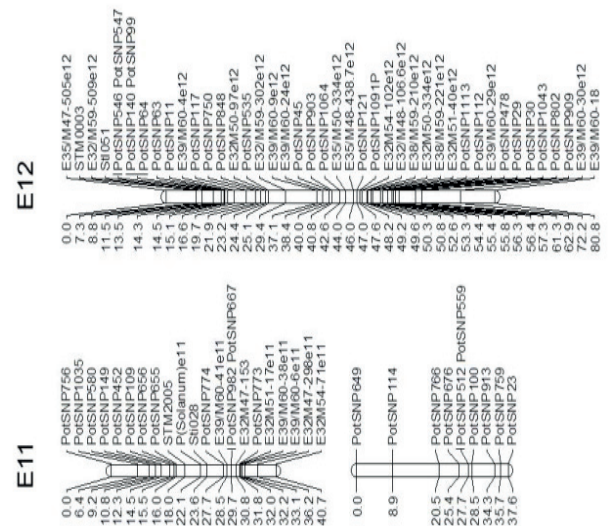

음

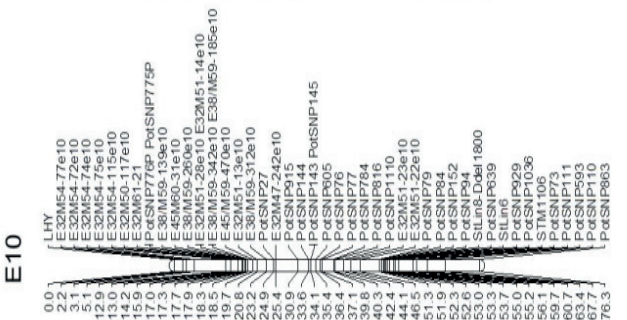

피

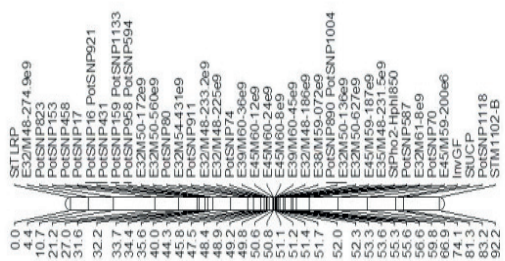

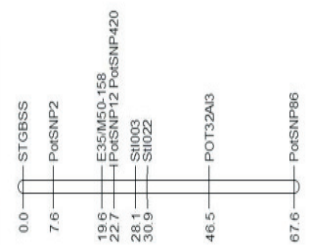

บ

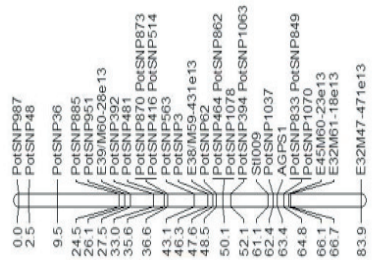

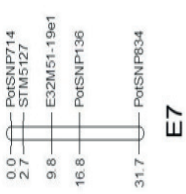
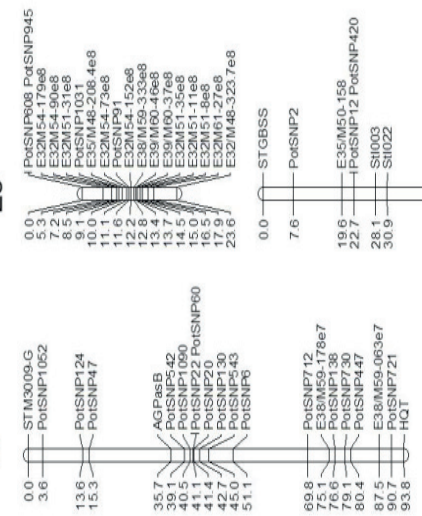


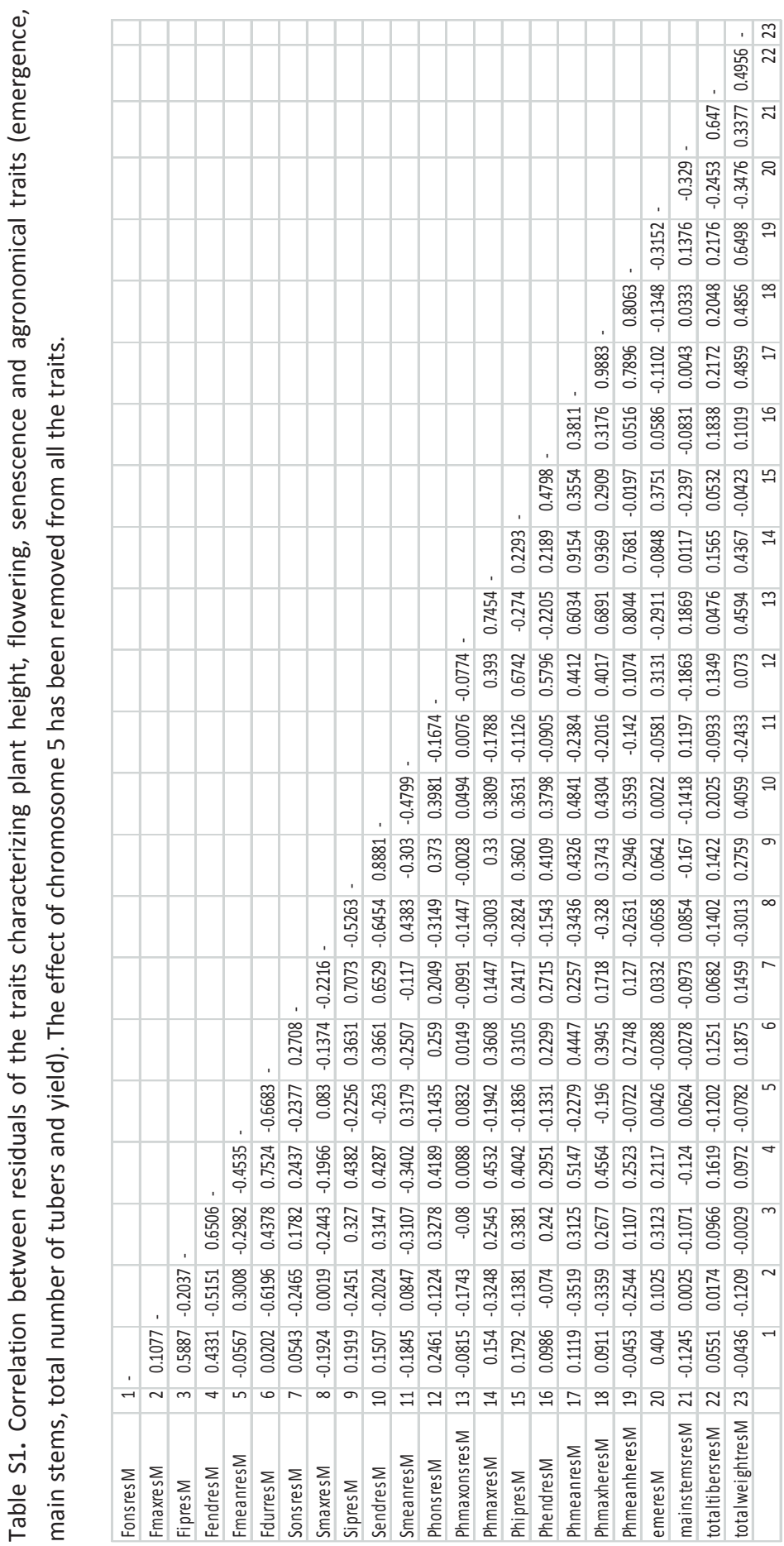


Table S2. QTL results of single trait linkage analysis using the characteristics describing Plant height (A), flowering (B) and senescence (C) and QTLs associated to the agronomical traits measured at harvest (D)

\section{A. Plant height}

\begin{tabular}{|l|l|l|r|r|r|r|r|}
\hline \multicolumn{1}{l|}{} & marker & LG & CM & (-Iog10P) & \%Expl & Add eff & s.e \\
\hline & E35/M47-345c5 & C5 & 75.03 & 3.071 & 4.535 & -0.332 & 0.098 \\
\hline & E39/M60-27e5 & E5 & 40.94 & 11.401 & 21.961 & -0.73 & 0.097 \\
\hline & E39/M60-4e12 & E12 & 16.57 & 4.731 & 7.691 & 0.432 & 0.098 \\
\hline maxons (slope) & Sti012m & C4 & 121.04 & 3.014 & 5.328 & 0.063 & 0.019 \\
\hline & PotSNP450 & C5 & 19.3 & 3.162 & 6.375 & -0.069 & 0.02 \\
\hline & Sti032f & E5 & 21.89 & 10.192 & 21.852 & -0.128 & 0.018 \\
\hline Max slope & PotSNP450 & C5 & 19.3 & 5.308 & 8.818 & -0.136 & 0.029 \\
\hline & Sti032m & C5 & 100.88 & 4.12 & 5.538 & -0.108 & 0.027 \\
\hline Ipoint & Sti032f & E5 & 21.89 & 20.686 & 38.935 & -0.286 & 0.026 \\
\hline & Sti032f & E5 & 21.89 & 10.845 & 22.445 & -0.632 & 0.087 \\
\hline End & E39/M60-4e12 & E12 & 16.57 & 3.591 & 6.067 & 0.329 & 0.088 \\
\hline Mean slope & GP21_2007 & E5 & 17.97 & 6.8 & 15.602 & -0.511 & 0.093 \\
\hline & PotSNP706 & C1 & 10.29 & 3.131 & 3.682 & 0.051 & 0.015 \\
\hline & PotSNP450 & C5 & 19.3 & 3.838 & 5.551 & -0.063 & 0.016 \\
\hline & Sti032m & C5 & 100.88 & 3.023 & 3.563 & -0.051 & 0.015 \\
\hline & Mando & E5 & 18.79 & 23.461 & 44.482 & -0.179 & 0.015 \\
\hline Max height & PotSNP706 & C1 & 10.29 & 2.729 & 4.117 & 2.416 & 0.764 \\
\hline & PotSNP450 & C5 & 19.3 & 2.84 & 4.888 & -2.632 & 0.812 \\
\hline & E39/M60-27e5 & E5 & 40.94 & 13.585 & 27.368 & -6.229 & 0.746 \\
\hline meanheight & STM5127m & C1 & 7.66 & 3.775 & 4.678 & 1.253 & 0.325 \\
\hline & StPho1bm & C5 & 124.25 & 4.009 & 4.976 & -1.292 & 0.324 \\
\hline & Mando & E5 & 18.79 & 21.439 & 40.675 & -3.695 & 0.328 \\
\hline
\end{tabular}

\section{B. Flowering}

\begin{tabular}{|l|l|l|l|r|r|r|r|}
\hline trait & marker & LG & CM & $(-\mid$ log10P $)$ & $\%$ Expl & Add eff & s.e \\
\hline onset & - & & & & & & \\
\hline max slope & Sti032m & C5 & 100.88 & 6.238 & 10.745 & 0.261 & 0.05 \\
\hline & SPUD237 & E5 & 23.67 & 17.576 & 41.061 & 0.51 & 0.051 \\
\hline Ipoint & PotSNP1145 & E5 & 36.43 & 6.396 & 16.113 & -0.421 & 0.079 \\
\hline End & PotSNP450 & C5 & 19.3 & 2.454 & 4.677 & -0.242 & 0.082 \\
\hline & Sti032m & C5 & 100.88 & 2.777 & 4.719 & -0.243 & 0.076 \\
\hline & SPUD237 & E5 & 23.67 & 14.62 & 35.705 & -0.67 & 0.076 \\
\hline mean slope & - & & & & & & \\
\hline Duration Flow & Sti032m & C5 & 100.88 & 3.815 & 6.896 & -0.339 & 0.087 \\
\hline & Mando & E5 & 18.79 & 13.435 & 33.378 & -0.746 & 0.089 \\
\hline
\end{tabular}




\section{Senescence}

\begin{tabular}{|l|l|l|r|r|r|r|l|}
\hline trait & marker & LG & CM & $(-\log 10 P)$ & \%Expl & Add eff & s.e \\
\hline onset & R1f & E5 & 35.47 & 4.987 & 11.823 & -0.389 & 0.085 \\
\hline max slope & E39/M60-27e5 & E5 & 40.94 & 9.712 & 22.992 & 0.388 & 0.057 \\
\hline ipoint & Sti032m & C5 & 100.88 & 8.005 & 10.711 & -0.397 & 0.066 \\
\hline & Sti032f & E5 & 21.89 & 24.714 & 46.025 & -0.823 & 0.065 \\
\hline & PotSNP81 & E6 & 4.27 & 3.654 & 4.474 & 0.257 & 0.068 \\
\hline & PotSNP91 & E8 & 11.57 & 2.8 & 4.114 & 0.246 & 0.077 \\
\hline end & PotSNP125 & C5 & 106.9 & 9.833 & 15.146 & -0.683 & 0.099 \\
\hline & Mando & E5 & 18.79 & 25.252 & 47.066 & -1.204 & 0.094 \\
\hline & PotSNP486 & E6 & 2.94 & 3.162 & 3.541 & 0.33 & 0.095 \\
\hline & & & & & & & \\
\hline
\end{tabular}

D. Traits measured at harvest

\begin{tabular}{|l|l|l|r|l|r|r|r|r|}
\hline trait & marker & h2 & LG & LG & cM & LOD & \%Expl. Var & Add effect \\
\hline e mergence & PotSNP142 & 0.8093 & 10 & C10 & 11.13 & 5.308 & 13.548 & 0.647 \\
\hline & Stl022f & & 26 & E8A & 30.87 & 3.944 & 9.404 & -0.539 \\
\hline total tubers & PotSNP95 & 0.8777 & 3 & C3 & 104.97 & 3.835 & 11.355 & -1.891 \\
\hline & Sti032f & & 21 & E5 & 21.89 & 4.703 & 9.761 & -1.753 \\
\hline total weight & Sti032m & 0.8324 & 5 & C5 & 100.88 & 5.248 & 8.43 & -26.857 \\
\hline & Stl009f & & 16 & E1A & 35.07 & 3.889 & 5.793 & 22.263 \\
\hline & Sti032f & & 21 & E5 & 21.89 & 13.98 & 27.294 & -48.324 \\
\hline \# main stem & PotSNP621 & 0.8015 & 5 & C5 & 84.41 & 3.742 & 8.427 & 0.379 \\
\hline
\end{tabular}




\section{Chapter 3 \\ Multiple QTLs contribute to tolerance to drought in potato grown under field conditions}

Biructawit B. Tessema ${ }^{1,2}$, Gebremedhin Gebregiorgis ${ }^{3}$, Richard GF Visser ${ }^{1}$, C. Gerard van der Linden $^{1}$

${ }^{1}$ Plant Breeding, Wageningen University \& Research, PO Box 386, 6700 AJ Wageningen, the Netherlands

${ }^{2}$ The graduate school of Experimental Plant Sciences (EPS), Wageningen University, Wageningen, the Netherlands

${ }^{3}$ Ethiopian Institute of Agricultural Research (EIAR), Holetta Agricultural Center, Ethiopia 


\begin{abstract}
Potato is highly valued as a food security crop but is sensitive to drought stress. A relatively small reduction of the optimum amount of water can already result in a significant reduction in tuber yield. Therefore unraveling the genetic basis of drought tolerance is important in order to enhance tolerance to drought in potato. However, evaluating the genetic basis of drought tolerance traits in potato is complex since expression of quantitative traits is not only controlled by genetic components, but also by the environment in which the plants are growing. We have evaluated a diploid (CXE) potato backcross mapping population for drought tolerance under field conditions. Water application was completely withheld at the stage of tuber initiation. At the end of the drought stress period, we collected agromorphological and physiological traits. The potato population showed significant reduction in several growth traits measured as well as in tuber yield production. In order to find the genomic regions determining or influencing these drought tolerance traits, we applied quantitative trait locus (QTL) analysis. We found a total of 60 QTL for several of the traits measured under well-watered and drought stress conditions. From the total number of QTLS identified, 21 were detected under water stress conditions while 39 QTLs were identified under well-watered conditions. Most of the QTLs detected co-localized on chromosome 5, in the same location of a known major QTL for earliness. QTLs discovered for drought tolerance traits in this study can aid in improving potato for drought tolerance.
\end{abstract}

Key words: severe drought stress, QTL, potato, field 


\section{Introduction}

Potato is one of the most important non-grain food commodities worldwide. Potato has a shallow root system in which about $85 \%$ of the total root length is concentrated in the upper $0.3 \mathrm{~m}$ of soil, making potato sensitive to water shortage (Gregory and Simmonds 1992; Iwama 2008). However under well-watered conditions, potato stands out as an efficient water user compared to wheat, maize and rice producing significantly higher kcal per m3 water (Renault and Wallender 2000). The yield losses for potato due to drought stress are expected to increase, since water scarcity is predicted to be severe in most agricultural production areas in the coming decades (Hijmans 2003). Thus, a better understanding of drought responses helps in improving potato for water stress.

Plant response to drought stress is complex and involves several morphological and physiological adaptations. The response of water stress effects on potato depends on the phenological timing, duration and severity of the stress (Jefferies 1995a). Water shortage during tuber initiation stage of potato has a drastic effect on tuber yield and makes recovery even more difficult (Deblonde and Ledent 2001). Many authors have studied drought responses in potato in order to understand the underlying mechanisms of drought stress tolerance (Monneveux et al. 2013). The effect of water scarcity in potato ranges from reduced photosynthetic rate to induced canopy senescence with negative consequences on tuber yield and quality (Dalla Costa et al. 1997; Munné-Bosch and Alegre 2004).

The availability of genetic variation in wild potato germplasm allows for drought stress tolerance improvements (Schafleitner et al. 2007; Anithakumari et al. 2012). Such landraces are better adapted to harsh environments including water scarce conditions (Schafleitner et al. 2007), and genes from this germplasm could help improving cultivated potatoes that are considered susceptible to drought stress (Monneveux et al. 2013). Drought tolerance is a quantitative trait controlled by a number of genes and/or gene families. The mechanisms underlying drought tolerance in cultivated potato can be studied using progenies derived from interspecific crosses between drought tolerant and drought sensitive potato genotypes. 
However, there are only few studies describing the dissection of the genetic basis of drought tolerance in potato (Anithakumari et al. 2011; Anithakumari et al. 2012; Khan et al. 2015). Drought tolerance studies under in-vitro and green house conditions have led to the discovery of QTL for drought tolerance traits in diploid mapping populations, and some of the QTL were drought specific (Anithakumari et al. 2011; Anithakumari et al. 2012). In the study of Khan et al. (2015) QTL were detected for drought tolerance traits both under green house and field conditions with a potential to contribute to potato improvement.

In the present study, a diploid (CXE) potato backcross mapping population was used to dissect drought tolerance under field conditions. In a semi-arid environment, this potato population was exposed to drought stress starting from tuber initiation and drought tolerance traits were collected during the water stress period. QTL analysis was then applied to find the genomic regions controlling these drought tolerance traits.

\section{Materials and Methods}

In two consecutive years (2010: Experiment 1 and 2011: Experiment 2), a field experiment was carried out in a semi-arid environment at Melkassa, Ethiopia. The area is characterized by an average day temperature of $280 \mathrm{C}$, annual rain fall of $928 \mathrm{~mm}$ and is situated at 1550 meters above sea level (masl) at coordinates $8024^{\prime} \mathrm{N} 39021^{\prime} \mathrm{E}$, with clay loam soil. The weather characteerstics during the experiment period is given in table 1.

\section{Plant materials}

A set of 104 diploid potato mapping population from crosses of two diploid potato clones, USW53373.3 coded C and 77.2102.37 coded E was used. Clone C is a hybrid between S. phureja PI 225696.1 and the S. tuberosum dihaploid USW42. Clone E is a cross between clone $\mathrm{C}$ and the $\mathrm{S}$. vernei $-\mathrm{S}$. tuberosum backcross clone VH34211. The full description of the population can be obtained from (Celis-Gamboa 2002). The selected population largely overlaps with the set used by Anithakumari et al. (2012). Tetraploid potato cultivars commonly grown in Ethiopia (Awash, Belete, Bulle, Gera, Gudene, Jalene, Shenkola, and Zengena, or in the Netherlands (Bintje, Desiree, Gloria, Granola, Karnico, Premiere, and Saturna) were included in the experiments as well. 
Table 1. Wheather data collected during the field experiment at Melkassa

\begin{tabular}{llccc}
\hline Year & Parameters & September & October & November \\
\hline 2010 & $\begin{array}{l}\text { Minimum } \\
\text { Temperature }\left({ }^{\circ} \mathrm{C}\right)\end{array}$ & 15.2 & 11 & 10.8 \\
& $\begin{array}{l}\text { Maximum } \\
\text { Temperature }\left({ }^{\circ} \mathrm{C}\right)\end{array}$ & 27.6 & 29.9 & 28.7 \\
& $\begin{array}{l}\text { Relative humidity }(\%) \\
2011\end{array}$ & 68 & 46 & 50 \\
\hline & $\begin{array}{l}\text { Minimum } \\
\text { Temperature }\left({ }^{\circ} \mathrm{C}\right)\end{array}$ & 8.8 & 4.5 & 6.7 \\
& $\begin{array}{l}\text { Maximum } \\
\text { Temperature }\left({ }^{\circ} \mathrm{C}\right)\end{array}$ & 26.7 & 29 & 28 \\
& Relative humidity $(\%)$ & 70.7 & 41 & 53 \\
\hline
\end{tabular}

\section{Experimental design}

A split plot design with two blocks was used and the 104 CXE diploid potato genotypes together with Parents $C$ and $E$ were randomized within a block. The water regime was allocated to the main plot and genotypes were assigned to subplots with two replications. In each replication eight plants per genotype were planted and planting was done manually. The distance between and within row were $75 \mathrm{~cm}$ and $30 \mathrm{~cm}$, respectively. A split application of Urea at planting and flowering was done at a rate of $165 \mathrm{~kg} / \mathrm{ha}$ and Diammononium phosphate (DAP) was applied at planting at a rate of $195 \mathrm{~kg} / \mathrm{ha}$. Furrow irrigation was used to water the field with an average interval of four days. The four replicates were kept watered until tuber initiation, watering was ceased for two of the replications at the time of tuber initiation and the plants were without irrigation for 38 days in the first experiment and 50 days for the second experiment. For both years harvesting was done at the end of the drought stress period ( 73 and 75 days after planting for the first and second experiment, respectively).

All the field cultivation practices were done manually. The field was kept free of weeds by hand weeding whenever necessary. During the growing periods ridging of the field was done four times. The field was sprayed with Redomil Gold and Mancozeb to control late blight (Phytophthora infestance) infestations and applications were done six times. The chemical Selecron was sprayed against cut worm (Agrotis ipsilon), Agro-methiotate against potato 
tuber moth (Phthorimaea operculella), and Mancozeb against potato stem blight (Sclerotium rolfsii). All the diseases were controlled except for late blight disease. Late blight infection was scored using a disease scoring scale of the Centro Internacional de la Papa (CIP) technical bulletin.

\section{Phenotyping}

Data collection started on average 16 and 12 days after planting (DAP) by scoring emergence in 2010 and 2011 respectively. Emergenev scoring wascontinued with daily observations until all eight plants of each of the 104 genotypes and 17 cultivars had emerged. For the two experimental years, number of main stems was counted and plant height was measured from the tip of the plant to the soil level by selecting the longest main stem.

In the 2011 experiment, for shoot and root biomass measurements, shoots and roots were weighed immediately after harvest as fresh weight and after oven drying at $1050 \mathrm{C}$ for 48 hours as dry weight. For measuring root length, each side of the plant was carefully dug out to uproot the plant and roots were cleaned with water. The longest root length was then measured with a ruler. Tubers larger than $20 \mathrm{~mm}$ in diameter were counted and weighed as tuber number and yield. For tuber dry weight measurement, tubers were sliced and dried in an oven at $800 \mathrm{C}$ for 72 hours. Tuber yield data is available for both experimental years. Total fresh biomass (g), was calculated by adding fresh weight of shoot and tuber. Similarly, total dry biomass (g) was calculated. Harvest index based on dry weight was calculated by dividing tuber dry weight by total dry biomass. Root to shoot ratio (R:S) was calculated as the ratio of root dry weight and shoot dry weight.

For both experimental years, Chlorophyll content was measured at 19, 29 and 34 DAS (CC19DAS, CC29DAS and CC34DAS) with a SPAD 502 chlorophyll meter (Minolta Co., Japan). Measurements were done on the third leaflet from the top young fully expanded leaflet.

Despite the use of different fungicide chemicals, late blight disease could not be fully controlled. Drought-stressed plants were more affected than well-watered plants. The incidence level of the disease was scored on a scale from 1 - 9 following CIP guideline Henfling (1987). Disease score was done at different time points; 39 days after stress (DAS) 
for 2010 and 22, 33 and 44 days after stress for 2011. In order to account for the effect of disease effects possibly confounding the effects of the drought stress on the phenotypic traits, disease score (39 DAS for 2010 and 44 DAS for 2011) was used as a covariate in the analysis of variance.

\section{Statistical analysis}

All the data collected for both treatments were statistically analyzed using Genstat 15th edition (VSNi 2012). For correcting drought tolerance traits for disease effect, the disease incidence scored at 44DAS was used as a covariate in analysis of variance (ANCOVA), since it coincides with the timing of drought trait data collected at the end of stress. Broad sense

heritability $(\mathrm{H} 2)$ was computed as $\mathrm{H} 2=\sigma_{\mathrm{g}}^{2} /\left(\sigma_{\mathrm{g}}^{2}+\frac{\sigma_{\mathrm{e}}^{2}}{\mathrm{r}}\right)$, where $\sigma_{\mathrm{g}}^{2}$ is genetic variance, $\sigma_{\mathrm{e}}^{2}$ is environmental variance, and $r$ is number of replications. Relative reduction of traits was calculated as a difference between control and drought which then divided by the control mean and expressed in terms of percentage.

\section{Genetic map construction}

The marker data used to construct the genetic map used in this study is described in Anithakumari et al. (2012). The marker data includes Amplified Fragment Length Polymorphism (AFLP), Simple Sequence Repeats (SSR), Cleavage Amplified Polymorphism (CAP) and Single Nucleotide Polymorphism (SNPs). The genetic map order and positions developed by Anithakumari et al. (2012) were used to reconstruct a genetic map for the CXE progeny used in this study. Joinmap 4.1 (Van Ooijen 2006) was used to construct an integrated CXE genetic map. We have used 529 markers in total and markers with LOD value above 5 were included for map construction.

\section{QTL analysis}

QTL analysis was done separately for control and drought stress treatments using MapQTL 6 (Van Ooijen 2009). Interval mapping was done first to identify and locate QTL on the linkage group. A permutation test was performed to determine significant QTL and a genome wide LOD threshold level of $\mathrm{P}<0.05$ was used to declare presence of significant QTLs. Following this, restricted multiple QTL mapping (rMQM) was done and markers for QTLs detected by 
interval mapping were used as a co-factor. This procedure was continued until a stable list of cofactors was obtained. The integrated maps and QTLs were drawn using MapChart 2.2 (Voorrips 2002).

\section{Results}

\section{Response to drought stress}

The field experiments were infected by late blight, which was difficult to control by chemical spraying. In order to see whether the effects of drought stress on growth and development of potato were confounded by the effects of the disease, phenotypic traits measured under drought stress conditions were corrected for the effects of late blight disease. The means of the genotypes for each collected trait were tested for significant difference before and after correcting for disease, and we found that none of the traits showed significant differences. Thus, the data correction for late blight did not significantly affect the phenotypic trait distribution and rank of genotypes. This confirmed that the effects of the disease on the phenotypic variation of the drought stress-related traits were not large. Frequency distribution showed a normal distribution for many of the traits measured under drought stress conditions, and some traits are shown in Figure 1. In many of the cases, both parents were in the middle of the distribution and the CxE progeny showed an extreme phenotypic variation indicating transgressive segregation.

The CxE potato mapping population showed a significant reduction in growth and yield in response to drought stress conditions (Table 2). The relative reduction of the measured traits due to water shortage ranged from 8 to $67 \%$. In both experiments, tuber weight was highly reduced (67 and 57\%, respectively) in response to water stress, showing the significant impact of water shortage on tuber yield. Similarly, biomass production was reduced by half in response to drought stress. Plant height was reduced by 26.14 and $22.59 \%$ in 2010 and 2011 experimental years, respectively. The lowest relative reduction in both experimental years due to drought stress was observed for stem numbers, indicating drought stress has little effect on stem number once stems are formed.

In two successive field experiments, analysis of variance revealed highly significant variation for almost all traits measured under drought stress and control conditions (Table 2). Highly 
significant variation $(\mathrm{P}<0.001)$ among genotypes was observed for the above ground growth traits stem number, plant height, shoot fresh and dry weight under drought stress and wellwatered conditions. Statistically significant variation was observed for below-ground traits root fresh and dry weight, and root length for both treatment conditions. Total fresh and dry biomass produced under drought stress conditions showed highly significant genotypic differences. Genotypes under water-limited conditions were significantly different for tuber number and tuber fresh and dry weight. The CxE genotypes were significantly different for harvest index based on dry weight only under well-watered conditions. Genotypes also showed significant variation for chlorophyll content measured at 19, 29 and 34 DAS, under both treatment conditions.

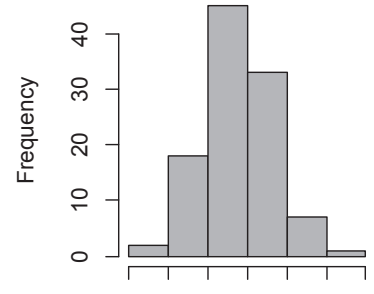

$\begin{array}{llll}0 & 10 & 30 & 50\end{array}$

Plant height $(\mathrm{cm})$

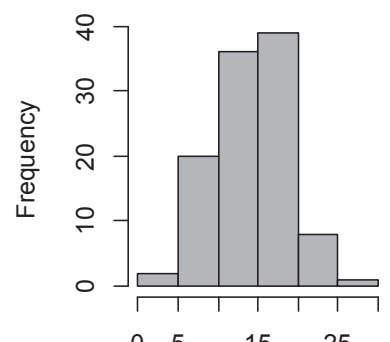

$0 \quad 5 \quad 15 \quad 25$

Tuber number

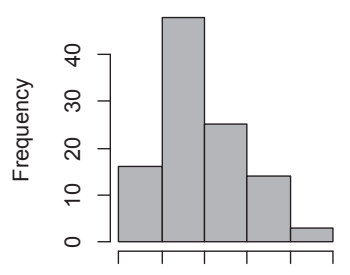

$\begin{array}{llllll}5 & 10 & 15 & 20 & 25 & 30\end{array}$

Root length (cm)

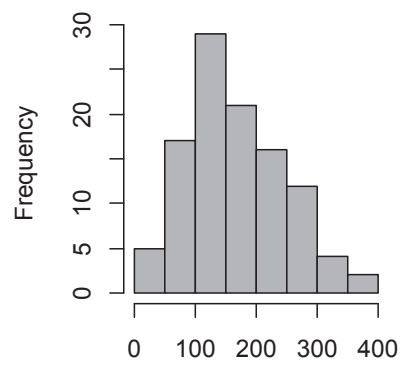

Tuber fresh weight (g)

Figure 1. Frequency distribution of some of the traits measured under drought conditions at the end of the stress period for the 2011 experiment. 
Table 2. Mean values of traits measured at harvest both under drought stress and wellwatered conditions in two different years with their respective significance value, relative reduction (RR), and broad sense heritability $\left(\mathrm{H}^{2}\right)$.

\begin{tabular}{|c|c|c|c|c|c|c|c|c|}
\hline \multirow[t]{2}{*}{ Traits } & \multirow[t]{2}{*}{ Year } & \multicolumn{3}{|c|}{ Drought stress } & \multicolumn{3}{|c|}{ Well-watered } & \multirow[t]{2}{*}{$\mathrm{RR}(\%)$} \\
\hline & & Mean & Pvalue & $\mathrm{H}^{2}$ & Mean & Pvalue & $\mathrm{H}^{2}$ & \\
\hline \multirow[t]{2}{*}{ Stem number } & 2010 & 2.6 & $<0.001$ & 0.52 & 3.3 & 0.002 & 0.45 & 15.00 \\
\hline & 2011 & 5.5 & $<0.001$ & 0.65 & 6.1 & $<0.001$ & 0.56 & 8.01 \\
\hline \multirow[t]{2}{*}{ Plant height $(\mathrm{cm})$} & 2010 & 26.5 & $<0.001$ & 0.62 & 40.2 & $<0.001$ & 0.76 & 26.14 \\
\hline & 2011 & 28.1 & $<0.001$ & 0.77 & 37.8 & $<0.001$ & 0.80 & 22.59 \\
\hline Shoot fresh weight (g) & 2011 & 97.6 & $<0.001$ & 0.61 & 233.8 & $<0.001$ & 0.76 & 46.47 \\
\hline Shoot dry weight (g) & 2011 & 14.3 & $<0.001$ & 0.68 & 31.1 & $<0.001$ & 0.71 & 42.15 \\
\hline Root fresh weight(g) & 2011 & 13.7 & 0.003 & 0.42 & 19.6 & $<0.001$ & 0.77 & 22.14 \\
\hline Root dry weight(g) & 2011 & 1.8 & $<0.001$ & 0.65 & 2.7 & $<0.001$ & 0.66 & 17.38 \\
\hline Root length $(\mathrm{cm})$ & 2011 & 14.8 & $<0.001$ & 0.94 & 23.4 & 0.003 & 0.42 & 37.07 \\
\hline Root:shoot (dry weight) & 2011 & 0.14 & 0.017 & 0.35 & 0.09 & $<0.001$ & 0.58 & -64.11 \\
\hline Total fresh biomass (g) & 2011 & 266.5 & $<0.001$ & 0.70 & 654.3 & $<0.001$ & 0.91 & 55.44 \\
\hline Total dry biomass (g) & 2011 & 53.7 & $<0.001$ & 0.51 & 114.9 & $<0.001$ & 0.80 & 41.14 \\
\hline \multirow[t]{2}{*}{ Tuber number } & 2010 & 7.9 & $<0.001$ & 0.62 & 16.8 & $<0.001$ & 0.75 & 44.57 \\
\hline & 2011 & 14.1 & $<0.001$ & 0.50 & 22.0 & $<0.001$ & 0.61 & 15.81 \\
\hline \multirow[t]{2}{*}{ Tuber fresh weight(g) } & 2010 & 88.2 & $<0.001$ & 0.53 & 369.3 & $<0.001$ & 0.68 & 67.00 \\
\hline & 2011 & 168.1 & $<0.001$ & 0.64 & 420.4 & $<0.001$ & 0.96 & 56.91 \\
\hline Tuber dry weight (g) & 2011 & 39.1 & 0.034 & 0.30 & 83.7 & $<0.001$ & 0.74 & 34.51 \\
\hline $\begin{array}{l}\text { Harvest index dry } \\
\text { weight }\end{array}$ & 2011 & 0.73 & 0.404 & 0.00 & 0.73 & 0.011 & 0.37 & -1.20 \\
\hline \multirow[t]{2}{*}{ CC19DAS } & 2010 & 49.4 & $<0.001$ & 0.58 & 47.1 & $<0.001$ & 0.69 & -5.20 \\
\hline & 2011 & 49.2 & $<0.001$ & 0.73 & 48.4 & $<0.001$ & 0.79 & -1.89 \\
\hline \multirow[t]{2}{*}{ CC29DAS } & 2010 & 49.8 & $<0.001$ & 0.54 & 46.8 & $<0.001$ & 0.63 & -7.00 \\
\hline & 2011 & 45.8 & $<0.001$ & 0.66 & 43.2 & $<0.001$ & 0.78 & -6.27 \\
\hline \multirow[t]{2}{*}{ CC34DAS } & 2010 & 46.9 & 0.004 & 0.44 & 44.8 & $<0.001$ & 0.59 & -5.58 \\
\hline & 2011 & 44.3 & $<0.001$ & 0.81 & 42.7 & $<0.001$ & 0.82 & -3.95 \\
\hline
\end{tabular}

\section{Heritability}

Broad sense heritability estimates of traits measured under drought and control conditions are presented in Table 2. The estimates of heritability varied over treatment, and ranged from moderate to high under both treatment conditions in both experimental years. Highest heritability under stress was observed for root length (0.94) and for tuber fresh weight (0.96) under control condition. The heritability for shoot fresh and dry weight under water stress conditions was 0.59 and 0.68 , respectively. Under water stress conditions, root to shoot 
ratio and tuber dry weight had low heritability. A moderate heritability was seen for stem number, root length and tuber dry matter content under well-watered conditions. Heritability was high for chlorophyll content measured in 2011 at all-time points, but decreased in the 2010 experiment with prolonged stress.

\section{Correlations among traits}

Phenotypic correlation coefficients for traits measured under well-watered and drought stress conditions are shown in Figure 2. Under stress conditions, plant maturity was positively correlated with shoot fresh weight, shoot dry weight and tuber yield. This implies that genotypes that matured late had higher shoot biomass and tuber yield. Under wellwatered conditions plant maturity also showed positive correlations with tuber yield, shoot fresh and dry biomass. Root length, root fresh and dry weight had significant positive correlation with tuber yield indicating genotypes with strong root expansion had better tuber yield. This correlation under well-watered condition was also significant. Moreover, tuber yield under water stress and well- watered conditions showed significant correlation with plant height, total fresh and dry biomass. Remarkably, relatively high correlations were found for tuber number with a number of growth traits under well-watered conditions, while these correlations were much lower under drought stress. On the other hand, tuber number had a higher significant correlation with root length under water stress conditions than under control conditions. Under drought stress conditions, tuber dry weight had significant positive correlations with several of the traits, including plant height, shoot fresh and dry weight, root dry weight, total fresh and dry biomass, and tuber number. Harvest index based on dry weight measured under water limited conditions exhibited a stronger significant negative correlation with root traits than under well-watered conditions. Root to shoot ratio was significantly correlated to harvest index under control conditions, but not anymore under water-limiting conditions, also indicating that the investment in roots did not result in higher tuber yield. The differential response of the root to shoot ratio was illustrated by the relatively poor correlation between control and drought for this trait (0.29). Harvest index had the lowest correlation between drought and control treatment (0.12), indicating a strong treatment by genotype effect. Another trait with relatively poor correlation between drought and control is tuber number (0.31), lower than fresh and dry 
tuber weight ( 0.71 and 0.56 , respectively), which may indicate a specific effect of drought on tuberization while tuber bulking is much less affected.

\begin{tabular}{|c|c|c|c|c|c|c|c|c|c|c|c|c|c|c|c|c|c|c|}
\hline PM & 1.00 & 0.55 & 0.59 & 0.59 & 0.67 & 0.65 & 0.47 & 0.61 & 0.56 & 0.54 & 0.51 & 0.28 & 0.15 & -0.28 & -0.25 & -0.01 & -0.20 & -0.15 \\
\hline $\mathrm{PlHt}$ & 0.60 & 0.68 & 0.80 & 0.80 & 0.60 & 0.74 & 0.53 & 0.78 & 0.79 & 0.66 & 0.73 & 0.55 & 0.23 & -0.32 & -0.47 & 0.05 & -0.17 & -0.09 \\
\hline SDW & 0.67 & 0.78 & 0.97 & 0.52 & 0.74 & 0.85 & 0.56 & 0.89 & 0.88 & 0.70 & 0.78 & 0.64 & 0.23 & -0.41 & -0.50 & 0.09 & -0.11 & -0.03 \\
\hline RDW & 0.61 & 0.69 & 0.78 & 0.80 & 0.75 & 0.77 & 0.63 & 0.78 & 0.83 & 0.61 & 0.77 & 0.54 & 0.19 & -0.23 & 0.22 & 0.03 & -0.13 & -0.06 \\
\hline RL & 0.49 & 0.57 & 0.71 & 0.74 & 0.62 & 0.55 & 0.49 & 0.54 & 0.56 & 0.45 & 0.52 & 0.38 & 0.10 & -0.16 & -0.07 & 0.07 & -0.05 & 0.01 \\
\hline TFB & 0.63 & 0.81 & 0.89 & 0.86 & 0.62 & 0.71 & 0.67 & 0.71 & 0.92 & 0.94 & 0.87 & 0.72 & 0.26 & -0.18 & -0.48 & 0.07 & -0.09 & -0.07 \\
\hline Tudwt & 0.47 & 0.76 & 0.68 & 0.64 & 0.43 & 0.58 & 0.55 & 0.85 & 0.94 & 0.87 & 0.56 & 0.74 & 0.24 & 0.16 & -0.31 & 0.10 & -0.05 & -0.06 \\
\hline TuNr & 0.16 & 0.09 & 0.10 & 0.13 & 0.10 & 0.07 & 0.65 & 0.20 & 0.20 & 0.25 & 0.22 & 0.31 & 0.39 & 0.10 & -0.21 & 0.16 & -0.03 & 0.07 \\
\hline StNr & 0.02 & 0.10 & 0.08 & 0.03 & -0.02 & -0.06 & 0.09 & 0.08 & 0.06 & 0.19 & 0.15 & 0.17 & 0.73 & -0.03 & -0.07 & 0.10 & 0.04 & 0.09 \\
\hline Hidry & -0.44 & -0.25 & -0.45 & -0.56 & -0.43 & -0.40 & -0.36 & -0.20 & -0.12 & 0.02 & 0.10 & -0.02 & 0.04 & 0.12 & 0.43 & 0.01 & 0.21 & 0.01 \\
\hline $\mathrm{R}: \mathrm{Sh}$ & -0.17 & -0.23 & -0.29 & -0.32 & -0.06 & 0.15 & -0.31 & -0.29 & -0.31 & -0.24 & -0.23 & -0.12 & -0.04 & 0.16 & 0.29 & -0.04 & 0.08 & 0.06 \\
\hline
\end{tabular}

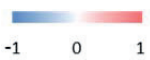

Figure 2. Pearson correlation showing relationships of traits measured at the end of drought stress period under drought stress (lower triangle) and well-watered (upper triangle) conditions. The diagonal indicates the correlation between the two different environments

$\mathrm{PM}=$ plant maturity, StNr= stem number, $\mathrm{PlHt}=$ plant height, SFW= shoot fresh weight, SDW= shoot dry weight, $\mathrm{RFW}=$ root fresh weight, $\mathrm{RDW}=$ root dry weight, $\mathrm{RL}=$ root length, $\mathrm{R}: \mathrm{Sh}=$ root to shoot ratio (dry weight), $T F B=$ total fresh biomass, $\mathrm{TDB}=$ total dry biomass, $\mathrm{TuNr}=$ tuber number, TuFWt= tuber fresh weight, TuDWt= tuber dry weight, HIdryW= harvest index dry weight, and CC19DAS, CC29DAS and CC34DAS is chlorophyll content measured on 19, 29, and 34 days after stress.

\section{QTL mapping}

A QTL analysis was done to unravel the genetic basis of the phenotypic variation observed in the CxE population under drought stress and well-watered conditions. In two successive field experiments, a total of 60 QTLs were detected for agronomical, morphological and physiological traits (Table 3; Figure 4). These QTLs were found on all the different chromosomes, except on chromosome 4. Most of the QTLs detected were for the 2011 experiment since several of the traits were measured for this year only. For well-watered 
treatment 39 QTLs were detected while under drought stress conditions, 21 QTLs were found. The phenotypic variation explained by the QTLS detected under water stress conditions ranged from 7.5 to $55.2 \%$, while QTLs identified under well-watered conditions explained 7.0 to $45.7 \%$ of the phenotypic variations observed. Under both treatment conditions several of the QTLs were located on chromosome 5 and many of the QTLS colocalized in the 26.2-44.0 cM interval, while one QTL identified under well-watered conditions for harvest index (dry weight) was located in the interval 52.4-66.5cM. These two different locations on chromosome 5 were also identified to contain QTL under drought stress and well-watered conditions in a greenhouse experiment (Anithakumari et al. 2012), and an expression QTL hotspot (40-50 cM) was identified on chromosome 5 for drought stress conditions (Muijen et al. 2016).

QTLs accumulating in the 26.2-44.0cM interval on chromosome 5 included QTLs for plant height under stress in both experimental years. For this trait, a QTL on chromosome 8 was identified for 2010 only that explained $13.7 \%$ of phenotypic variation. Further QTLS accumulating in the same region on chromosome 5 included QTLs for shoot fresh weight and shoot dry weight for drought stress treatment with high LOD scores of 16.58 and 17.64, accounting for 52 and $54.2 \%$ of phenotypic variation, respectively. Under drought stress treatment, identified QTLs for root fresh weight and dry weight in this region had a LOD score of 10.48 and 13.72, explaining 37.1 and $45.5 \%$ of observed phenotypic variation. Moreover, QTLs responsible for total fresh biomass with LOD values of 16.54 and total dry biomass with LOD values of 18.16 were detected under water stress treatment. For tuber fresh weight, QTLs located on chromosome 5, 7, 9 and 12 were detected for stress condition. The highest phenotypic variation (28.6\%) was explained by a QTL located in the 26.2-44.0 cM interval on chromosome 5. QTLs found on chromosome 7, 9, and 12 were specific to the drought stress condition.

For well-watered treatment, we detected QTLs on chromosome 5 for stem number, plant height, shoot fresh weight and dry weight, root fresh and dry weight, root length, total fresh and dry biomass, tuber fresh and dry weight, and tuber number. All these QTLs fall in the interval range of 26-47.3 cM. A QTL for harvest index based on dry weight falls in the interval 
of 52.4-62.9 cM (Figure 4). This suggests there may be two independent QTL regions located on chromosome 5, in agreement with Anithakumari et al. (2012) and Muijen et al. (2016).

Twelve significant QTLs were detected on other chromosomes than chromosome 5 for some of the traits measured under well-watered treatment, specifically on chromosome 1, 3, 6, 7, 9, and 11. A QTL on chromosome 1 was identified for root dry weight with LOD value of 4.71 and accounting for 7\% of phenotypic variation. On chromosome 3 and 7 QTLs for tuber number were identified that explained 11.8 and $15.3 \%$ of variance, respectively. QTLs for root dry weight, tuber number and tuber fresh weight co-localized on chromosome 8. QTLS for plant height were detected on chromosome 6 for both experimental years. Chromosome 9 had a QTL for stem number with LOD value of 4.72 that explained $15.5 \%$ of the observed variation. QTLs for shoot fresh weight and total fresh biomass were co-located on chromosome 11 , explaining 10.6 and $9.3 \%$ of phenotypic variation, respectively.

Several genomic regions responsible for the variation in chlorophyll content were found on chromosome 1, 2, 3, 7, 10, and 11, for different time points and both under drought stress and well-watered conditions. The QTL detected on chromosome 2 was present across years and treatments. This QTL falls in the interval from 79.9 to $109.3 \mathrm{cM}$ and the phenotypic variation explained ranged from 14.3-27.7\%. In addition, a QTL expressed across treatments and years was identified on chromosome 10, located between 20 and 46 cM. For wellwatered conditions, a QTL on chromosome 1 was found expressed across years. On chromosome 11, QTL region was detected for control treatment. QTLs on chromosome 3 and 7 were identified for chlorophyll content measured under drought stress conditions, and these explained 13.9 and $9.3 \%$ of the phenotypic variation, respectively. 
Table 3. QTLs detected for listed traits under drought stress (DS) and well-watered (WW) conditions with their linkage groups (LG), 2- LOD support value, intervals (cM), and explained variation (\%).

\begin{tabular}{|c|c|c|c|c|c|c|c|c|}
\hline Trait & year & treatment & QTL name & LG & Marker & LOD & interval & $\%$ Variation \\
\hline \multirow[t]{3}{*}{ Stem number } & 2010 & WW & StNr10 & CE5 & Mando & 5.97 & $26.0-40.0$ & 21.1 \\
\hline & & WW & StNr10 & CE9 & PotSNP594 & 4.72 & $53.5-59.9$ & 15.5 \\
\hline & 2011 & WW & StNr11 & CE5 & SPUD237 & 8.7 & $33.7-43.0$ & 30.8 \\
\hline \multirow[t]{7}{*}{ Plant height } & 2010 & WW & PlHt10 & CE5 & PotSNP697 & 16.18 & $31.2-47.3$ & 44.9 \\
\hline & & WW & PlHt10 & CE6 & PotSNP486 & 8.05 & $71.5-77.0$ & 14.7 \\
\hline & 2010 & DS & PlHt10 & CE5 & GP21_2007 & 9.83 & $27.0-38.0$ & 33.2 \\
\hline & & DS & PlHt10 & CE8 & PotSNP1067 & 4.71 & $1-18.6$ & 13.7 \\
\hline & 2011 & DS & PlHt11 & CE5 & SPUD237 & 16.29 & $32.2-44.0$ & 51.4 \\
\hline & 2011 & WW & PlHt11 & CE5 & PotSNP697 & 15.75 & $31.2-46.3$ & 42.8 \\
\hline & & WW & PlHt11 & CE6 & PotSNP150 & 5.21 & $75.6-79.1$ & 10.4 \\
\hline \multirow[t]{3}{*}{ Shoot fresh weight } & 2011 & DS & SFW11 & CE5 & SPUD237 & 16.58 & $33.7-43.0$ & 52 \\
\hline & 2011 & WW & SFW11 & CE5 & Mando & 10.84 & $31.2-42.0$ & 31.5 \\
\hline & & WW & SFW11 & CE11 & PotSNP100 & 4.48 & $25.6-38.8$ & 10.6 \\
\hline \multirow[t]{2}{*}{ Shoot dry weight } & 2011 & DS & SDW11 & CE5 & SPUD237 & 17.64 & $32.7-43.0$ & 54.2 \\
\hline & 2011 & WW & SDW11 & CE5 & Mando & 10.47 & $30.8-44.0$ & 37.4 \\
\hline \multirow[t]{2}{*}{ Root fresh weight } & 2011 & DS & RFW11 & CE5 & Mando & 10.48 & $30.0-39.0$ & 37.1 \\
\hline & 2011 & WW & RFW11 & CE5 & Mando & 13.8 & $31.2-41.0$ & 45.7 \\
\hline \multirow[t]{4}{*}{ Root dry weight } & 2011 & DS & RDW11 & CE5 & Mando & 13.72 & $31.2-37.7$ & 45.5 \\
\hline & 2011 & WW & RDW11 & CE1 & PotSNP72 & 4.71 & $\begin{array}{l}88.0- \\
114.9\end{array}$ & 7 \\
\hline & & WW & RDW11 & CE5 & Mando & 14.62 & $32.2-36.7$ & 37 \\
\hline & & WW & RDW11 & CE8 & STM1024 & 4.86 & $35.7-37.5$ & 7.5 \\
\hline \multirow[t]{2}{*}{ Root length } & 2011 & DS & RL11 & CE5 & SUPD237 & 8.36 & $31.2-44.0$ & 30.9 \\
\hline & 2011 & WW & RL11 & CE5 & Mando & 11.29 & $28.0-38.0$ & 39.3 \\
\hline \multirow[t]{3}{*}{ Total fresh biomass } & 2011 & DS & TFB11 & CE5 & SPUD237 & 14.45 & $32.2-44.0$ & 47.3 \\
\hline & 2011 & WW & TFB11 & CE5 & Mando & 13.74 & $31.2-40.4$ & 39.6 \\
\hline & & WW & TFB11 & CE11 & PotSNP100 & 4.36 & $\begin{array}{c}22.6- \\
38.8\end{array}$ & 9.3 \\
\hline \multirow[t]{2}{*}{ Total dry biomass } & 2011 & DS & TDW11 & CE5 & SPUD237 & 15.61 & $32.7-44.0$ & 49.6 \\
\hline & 2011 & WW & TDW11 & CE5 & Mando & 10.7 & $30.0-43.0$ & 37.7 \\
\hline \multirow[t]{4}{*}{ Tuber number } & 2010 & WW & TuNr11 & CE3 & PotSNP154 & 4.93 & $79.5-96.6$ & 11.8 \\
\hline & & WW & TuNr11 & CE7 & PotSNP712 & 5.43 & $20-54.9$ & 15.3 \\
\hline & & WW & TuNr11 & CE8 & STM1024 & 5.79 & $26.5-41.4$ & 14.6 \\
\hline & 2011 & WW & TuNr11 & CE5 & SPUD237 & 3.6 & $26.0-49.5$ & 14.7 \\
\hline
\end{tabular}




\begin{tabular}{|c|c|c|c|c|c|c|c|c|}
\hline Trait & year & treatment & QTL name & LG & Marker & LOD & interval & \%Variation \\
\hline \multirow[t]{6}{*}{ Tuber fresh weight } & 2010 & WW & TuNr11 & CE8 & Stl022 & 4.31 & $24.5-41.4$ & 19.2 \\
\hline & 2011 & DS & TuFwt11 & CE5 & SPUD237 & 12.38 & $31.2-43.0$ & 28.6 \\
\hline & & DS & TuFwt11 & CE7 & PotSNP25 & 4.84 & 29.3-44.1 & 9 \\
\hline & & DS & TuFwt11 & CE9 & PotSNP587 & 4.83 & $27.1-35.7$ & 9 \\
\hline & & DS & TuFwt11 & CE12 & $\begin{array}{l}\text { E39/M60- } \\
30 \text { e12 }\end{array}$ & 4.98 & $1-21.6$ & 9.4 \\
\hline & & WW & TuFwt11 & CE5 & Mando & 9.52 & $30.0-43.0$ & 34.4 \\
\hline Tuber dry weight & 2011 & DS & TuDwt11 & CE5 & SPUD237 & 10.83 & $32.2-45.0$ & 38.1 \\
\hline $\begin{array}{l}\text { Harvest index(dry } \\
\text { weight) }\end{array}$ & & WW & TuDwt11 & CE5 & Mando & 8.38 & $29.0-43.0$ & 31 \\
\hline \multirow[t]{8}{*}{ CC19DAS } & 2011 & WW & HIdry11 & CE5 & E45M60-27h5 & 5 & $52.4-66.5$ & 19.9 \\
\hline & 2010 & WW & CC19DAS10 & CE1 & PotSNP833 & 5.77 & $52.6-60.3$ & 14.4 \\
\hline & & WW & CC19DAS10 & CE2 & Myb_h17 & 7.38 & $\begin{array}{l}91.5- \\
103.8\end{array}$ & 19.1 \\
\hline & & WW & CC19DAS10 & CE10 & PotSNP111 & 5.47 & $22.9-40.4$ & 14 \\
\hline & 2011 & WW & CC19DAS11 & CE2 & PotSNP668 & 7.86 & $83.3-101.4$ & 18.7 \\
\hline & & WW & CC19DAS11 & CE10 & PotSNP639 & 6.46 & $35.0-46.6$ & 14.8 \\
\hline & & WW & CC19DAS11 & CE11 & PotSNP991 & 6.45 & $13.6-31.0$ & 14.3 \\
\hline & 2011 & DS & CC19DAS11 & CE10 & STM0051 & 5.38 & $66.8-73.1$ & 21.2 \\
\hline \multirow[t]{4}{*}{ CC29DAS } & 2010 & WW & CC29DAS10 & CE1 & Wrky_M3 & 4.95 & $45.3-47.2$ & 15 \\
\hline & & WW & CC29DAS10 & CE2 & Myb_h17 & 5.92 & $90.6-104.8$ & 20.2 \\
\hline & 2011 & WW & CC29DAS11 & CE1 & PotSNP833 & 5.18 & $50.6-58.3$ & 16.4 \\
\hline & & WW & CC29DAS11 & CE2 & PotSNP108 & 4.75 & $79.9-109.3$ & 14.3 \\
\hline \multirow[t]{2}{*}{ CC34DAS } & 2010 & WW & CC34DAS10 & CE1 & Wrky_H13 & 5.14 & $51.6-59.3$ & 15 \\
\hline & & WW & CC34DAS10 & CE2 & Myb_h17 & 7.94 & $92.5-103.8$ & 27.7 \\
\hline \multirow[t]{5}{*}{ CC34DAS } & 2011 & DS & CC34DAS11 & CE2 & PotSNP668 & 8.98 & $87.0-100.4$ & 18.4 \\
\hline & & DS & CC34DAS11 & CE3 & PotSNP653 & 6.81 & $3.8-12.7$ & 13.9 \\
\hline & & DS & CC34DAS11 & CE7 & PotSNP542 & 4.94 & $88.0-107.7$ & 9.3 \\
\hline & & DS & CC34DAS11 & CE10 & PotSNP111 & 6.31 & $20-46.0$ & 12 \\
\hline & 2011 & WW & CC34DAS11 & CE2 & PotSNP703 & 4.18 & $40.8-51.1$ & 16.9 \\
\hline
\end{tabular}

\section{Discussion}

\section{Response to drought stress}

In the evaluation of 104 individuals of the diploid potato CxE mapping population for morphological, physiological and agronomical parameters for drought tolerance, we observed a significant effect of water shortage on tuber yield production, in agreement with several other drought tolerance studies (Levy et al. 1990; Gregory and Simmonds 1992; Jefferies and MacKerron 1993; Lahlou et al. 2003; Anithakumari et al. 2012). All measured growth traits showed a considerable reduction due to water limitation stress, with largest 
effects on tuber yield and shoot parameters. Jefferies (1995) indicated that the response to drought in potato can be influenced by the severity of water limitation stress and the developmental stage of potato. Since our drought tolerance evaluation was done by completely with-holding water (severe stress) starting from tuber initiation, our discussion will be in view of this scenario. Moreover, how potato responds to water shortage condition can be influenced by the maturity type (Deblonde et al. 1999; Anithakumari et al. 2012). In the current study, the positive correlation of maturity with tuber yield indicates that intermediate and late maturing genotypes had better tuber production than early ones under the Ethiopian growth conditions in the field trials. The CXE genotypes has been assessed for developmental traits under short day (Ethiopian) conditions and the life span of the genotypes was shorter compared to the longer life span observed under long day conditions (the Netherlands and Finland) (Hurtado et al. 2012). This indicates that short photoperiod has accelerated the development process through early onset of senescence. However, under both short and long day conditions early genotypes were observed to be early and late genotypes were also observed to be late, indicating earliness behavior of the genotypes were similar under different day light conditions. Under short day (Ethiopia) conditions genotypes with longer senescence period were reported to have higher tuber yield (Hurtado et al. 2012).

Drought stress occurring at the stage of tuber initiation can significantly reduce photosynthesis, biomass production and tuber yield (Dalla Costa et al. 1997). The strong positive correlation of above ground biomass with tuber yield and tuber dry weight in this study indicates that shoot biomass is an important indicator for yield both under wellwatered and drought conditions. Schittenhelm et al. (2006) have indicated that potato genotypes that can maintain above ground biomass under stress conditions are able to produce higher yields. The amount of shoot biomass produced can be influenced by maturity type, as we have observed significant positive correlations with plant maturity under both stressed and non-stressed conditions. 
CE1

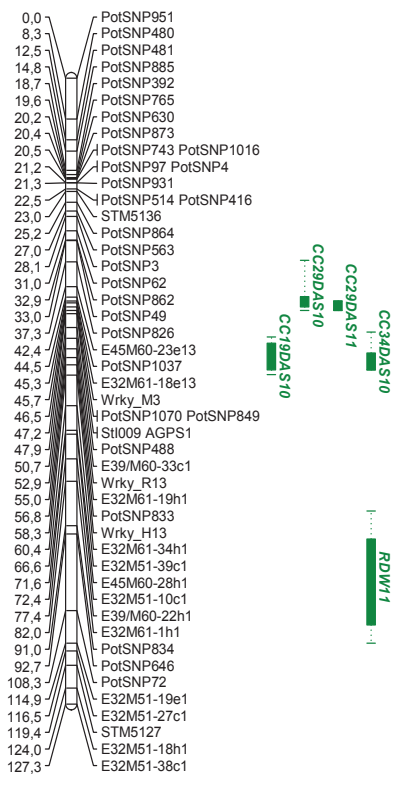

CE5
CE2

CE3

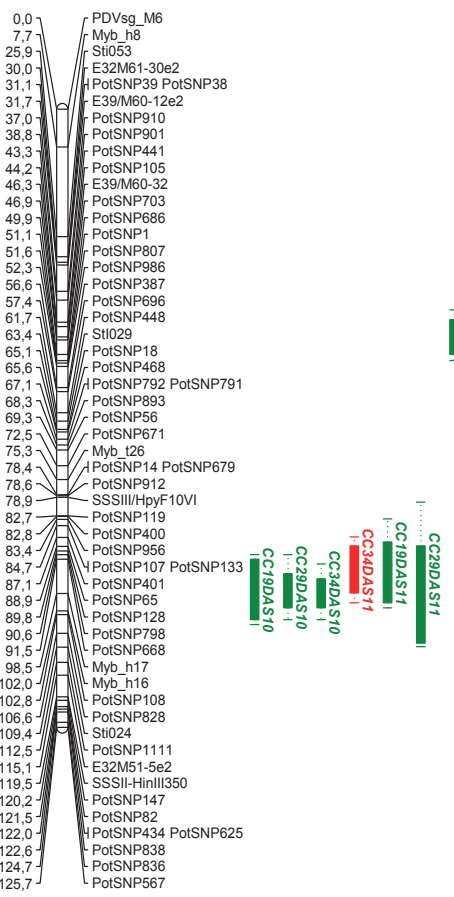

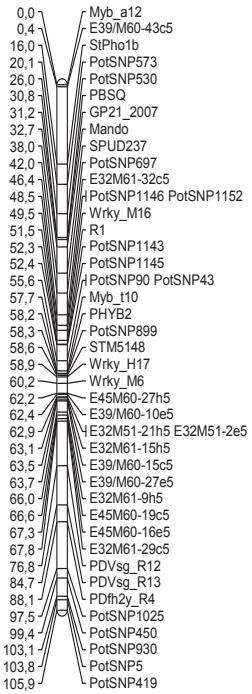

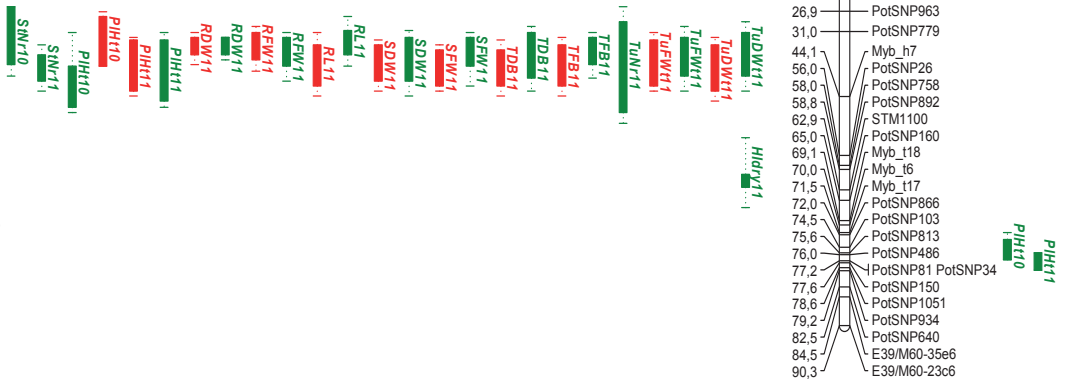




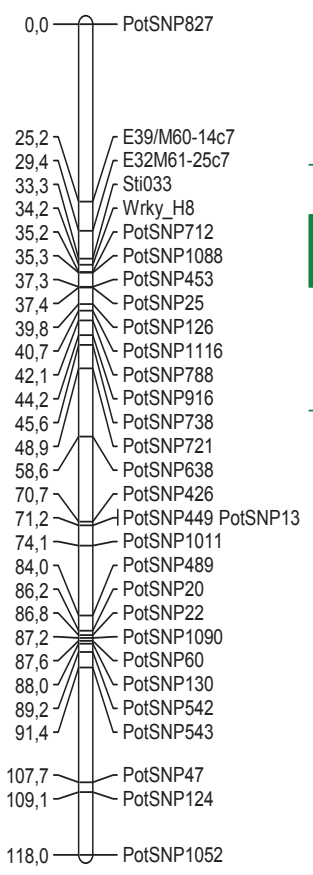

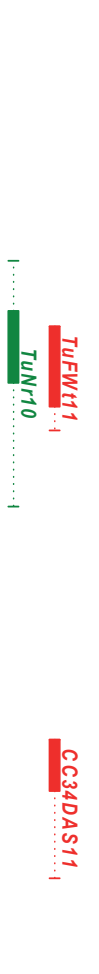

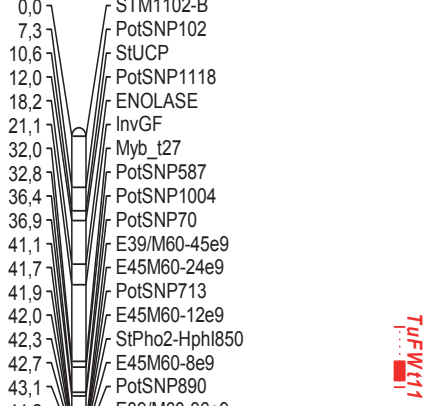

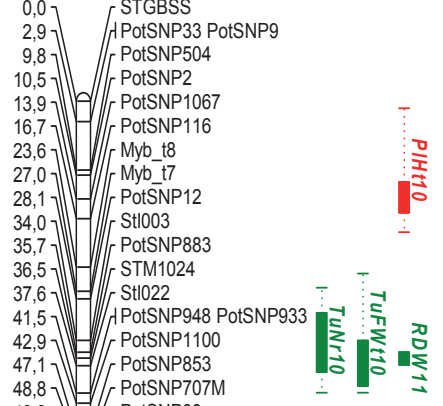

48,9 $]$ - PotSNP89

53,9 $\because$ РОT32AI1

55,1 St1027

$57,9-\mathrm{E} 45 \mathrm{M} 60-22 \mathrm{C8}$

60,6 PotSNP46

62,0 PotSNP155

69,3 PotSNP700

71,1 E32M61-14C8

75.9 PotSNP637

77,8 PotSNP104

78,1 PotSNP608

81,3-E32M51-15h8

81,8 E45M60-30c8

84,4 PotSNP821

87,4 PotSNP945

90,0 a Y PotSNP474 PotSNP749

93,7 1 Myb_t5 PotSNP91

94,9 7 [PDVsg_R1

97.2 [E32M51-31e8

97,8 $]$ - Wrky_R10

98,6] [E39/M60-46e8

99,6 $\triangle \backsim$ Wrky M14

99,8 E32M51-35e8

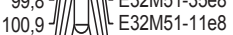

103,1 1 .

111,3 Wrky_M13

116,5] Wrky_H16

122,1 $\quad$ Wrky_R9

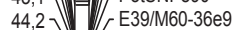

45,2 1 PotSNP74

46,5 $=$ PotSNP911

48,9- - E32M61-8e9

51,3 PotSNP80

53,4 1 E32M61-23c9

53,5]

$54,6]=$ E32M51-1C9

$58,2]$ H. PotSNP594

58,7 - PotSNP958

$59,9$ ] $)($ PotSNP1133

60,6 -

61,3 PotSNP17

61,8

61,9 4 PotSNP921 PotSNP431

65,8 - - PotSNP1115

69,2 -

71,8 ] PotSNP153

76,7 Myb +24

83,2 - PotSNP900

$\left.\begin{array}{l}83,2 \\ 84,8\end{array}\right]$ -

89,8 $]$ PotS̄NP50

96,5

97,0

101,5 $\quad$ Myb h10 

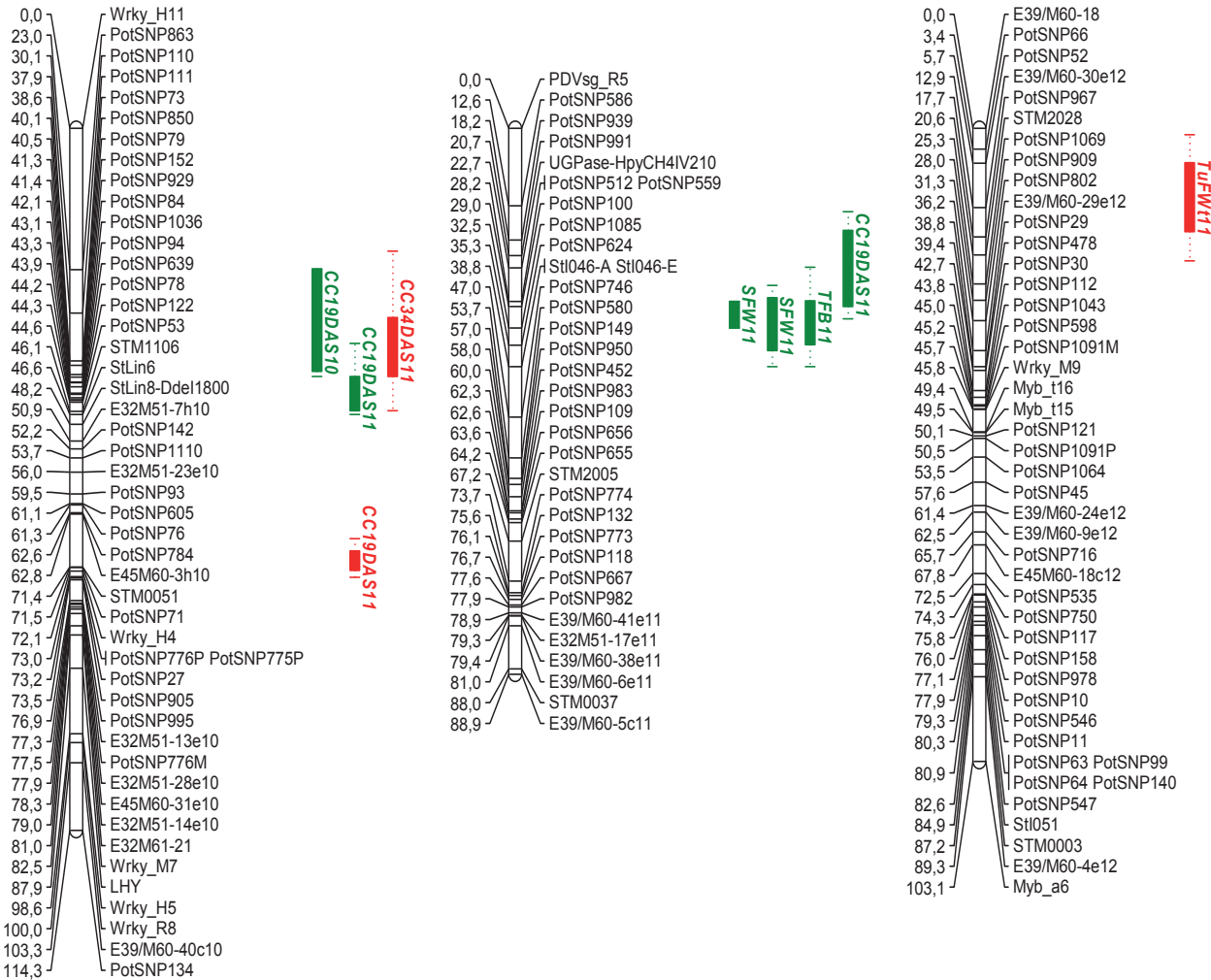

P POTSNP29

39,4 ] - PotSNP478

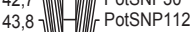

45,0] $]$ - $-\left[\begin{array}{l}\text { PotSNP1043 } \\ \text { PotSNP112 }\end{array}\right.$

45,2] - - [ PotSNP598

45,7] - PotSNP1091M

45,8 - Wrky M9

49,4 - Myb_t16

49,5 $=$ Myb_t15

50,1 PotSNP121

50,5 PotSNP1091P

53,5 PotSNP1064

57,6 PotSNP45

61,4 E39/M60-24e12

65,5 $\begin{aligned} & \text { E39/M60-9e12 } \\ & \text { PotSNP716 }\end{aligned}$

67,8 - E45M60-18c12

72,5

74,3 J组 (-PotSNP750

75,8 $]$ - PotSNP117

76,0 - $-\left(\begin{array}{l}\text { PotSNP158 } \\ \text { PotS }\end{array}\right.$

$77,1]-($ PotSNP978

77,9 - PotSNP10

79,3 $]$ PotSNP546

DotSNP63 PotSNP99

80,9 7 PotSNP64 PotSNP140

82,6 - PotSNP547

84,9 St1051

87,2 - STM0003

89,3 E39/M60-4e12

103,1 [Myb_a6

Figure 4. CE integrated linkage map with QTL detected under drought stress (red bar) and well-watered (green bar) conditions. Markers names are shown on the right side of the linkage group, with their positions indicated on the left. Bars indicate QTL, with the 2 LOD (Solid fill) and 1 LOD (dotted line) support interval indicated as well. Trait names and experimental year located on QTL bars, for trait names refer table 3.

\section{QTLs for drought tolerance}

Genetically dissecting drought tolerance in the CxE population has resulted in the discovery of 60 QTLs responsible for controlling agro-morphological and physiological traits under drought stress and well-watered conditions. The QTLs discovered under drought stress conditions can be used to enhance potato for tolerance to drought as we define tolerance the ability of genotypes to give higher yield under drought stress condition. As in the other 
drought studies (Anithakumari et al. 2012; Khan et al. 2015), chromosome 5 had a hot spot region for QTLs identified for several of the traits under drought stress and well-watered conditions. This locus on chromosome 5 was reported to control developmental traits (Plant height, flowering, and senescence) with the CxE population under short photoperiod conditions (Hurtado-Lopez et al. 2015). In this study, QTLs co-localizing on chromosome 5 were seen in two different positions, from the total 26 QTLs, 25 of the QTLs fell in the interval between 26.2 and $44.0 \mathrm{cM}$ and 1 QTL was located in interval range of 52.4 to 66.5 cM. The latter QTLs were identified only for well-watered treatment. QTLs on these two different positions on chromosome 5 were also reported by Anithakumari et al. (2012), where out of 21 QTLs detected 3 QTLs found under drought stress conditions were located in the interval range of 47 to $71 \mathrm{cM}$, while the rest of the QTLs for well-watered, stress and recovery were located between 20 and $48 \mathrm{cM}$. These results suggest there may be two different, independent QTL present on chromosome 5. For this same potato population, 31 QTLs controlling developmental (plant height, flowering and senescence) and 24 QTLs affecting agronomic traits were reported under short day condition (Hurtado-Lopez et al. 2015). Major QTL present on chromosome 5 had explained higher percentage (up to 60\%) of the phenotypic variation observed for developmental traits as reported in other studies (Celis-Gamboa 2002; Hurtado et al. 2012) and had explained lower (29\%) phenotypic variance for agronomic traits (tuber number and yield). This indicates that the major QTL located on chromosome 5 have higher influence in controlling developmental and agronomic traits under short day conditions.

Unlike the current study, only one QTL was reported on chromosome 5 for well-watered conditions by Anithakumari et al. (2012). Although both experiments were done using similar set of diploid potato population and genetic markers, the difference in the detected QTLs between these two studies could be attributed to the different experimental conditions (field and greenhouse). Besides a genetic component of a trait controlling its expression, environmental factors do have great influences on the expression of quantitative traits (Tuberosa 2012). This indicates selection for drought tolerance can be complex. Furthermore, a major QTL for earliness is located on chromosome 5 (Visker et al. 2003). The underlying gene of this major QTL was identified as a CDF gene with a pleiotropic effect on many agro-morphological traits of potato (Visker et al. 2003; Kloosterman et al. 2013). The 
CDF alleles control tuber initiation in potato, and also affect plant maturity. Other studies have identified QTLs on chromosome 5 for maturity, vigour and tuberization (Van den Berg et al. 1996; Danan et al. 2011; Collins et al. 1999).

The co-localization of QTLs for shoot and root traits with tuber yield on chromosome 5 indicates that the underlying gene(s) have pleiotropic effects (Figure 2). A QTL for tuber yield was found on chromosome 5 and explained $28.6 \%$ of observed phenotypic variation under water stress conditions. Similarly, a QTL for tuber yield on the same locus was reported under stress conditions in a greenhouse experiment (Anithakumari et al. 2012). These findings suggest chromosome 5 harbors important QTL that can be used in marker assisted breeding program to improve potato for drought tolerance. QTLs for root length, root fresh and dry weight co-located on chromosome 5 with plant height, shoot fresh and dry weight. The amount of variation explained by these QTLs ranged from 30.9 to $54.2 \%$. Root system architecture is considered important under water stress conditions as it plays a role in avoiding drought stress (Iwama 2008; Tuberosa 2012). Our results indicated that genotypes with strong root expansion had a better tuber yield production under both well-watered and under drought conditions, indicated by the significant positive correlation of root fresh and dry weight with tuber weight. Similarly, other studies have reported positive correlation of root dry mass with tuber yield under water stress (Lahlou and Ledent 2005). This suggests that root mass may be used as a selection criteria for improving drought tolerance in potato. However, measuring root traits can be difficult and tedious thus focusing on shoot traits that showed high correlation with root traits as an indirect selection criterion for root traits is very practical. Under stress conditions we found that plant height, shoot fresh and dry weight had showed high correlation with root length and root fresh and dry weight. Thus these triats can be used in enhancing potato for tolerance to drought.

A QTL associated with plant height located on chromosome 5 was found under water stress conditions and it had significant high correlation with tuber weight and tuber dry weight. Although we cannot establish this QTL as drought specific as it was also detected under wellwatered conditions, it still can be useful as a selection criterion for higher yields under water-limiting conditions. Similarly, a QTL for plant height was reported on chromosome 5 under stress conditions that where imposed at the later developmental stage of potato) in a 
field experiment with the diploid mapping population (Khan et al. 2015). This may suggest a locus located on chromosome 5 controls drought responses imposed both at early and late developmental stages of potato. Tourneux et al. (2003) have shown the correlation of plant height with tuber yield under drought stress conditions suggesting this as a morphological trait that can be used as a good indicator of tolerance to drought in potato. Unlike in our study, Anithakumari et al. (2012) reported QTL for plant height under stress conditions on chromosome 2 and 7. However, it is important to account the environmental difference (temperature, relative humidity, soil compositions, etc.) of the experiment that could result in significant differences of QTL detected for similar traits, as the expression of quantitative trait is not only determined by its genetic component but also by the environment in which it is growing. In our study, the maximum temperature recorded was $29.90 \mathrm{C}$ and $290 \mathrm{C}$ for the two successive year experiment (Table 1) while Anithakumari et al. (2012) reported 330C and $37.90 \mathrm{C}$ for their experiment period. Besides, in our experiment other factors such as day length was short and growing medium was field unlike Anithakumari et al. (2012) experiment where day length was short and pot was used as growing medium. It has been reported that shorter day length could restrict plant height growth compared to longer day length under normal field conditions (Paula 2012). Therefore such differences may account for the differences in QTLs expressed under different environmental conditions.

Shoot traits showed high correlation with tuber fresh and dry weight under water stress conditions, which may put them as potential drought tolerance indicator traits that can be used in potato improvement program. Furthermore, measuring shoot traits is easy, direct, and inexpensive. This may suggest canopy measurement can be used as selection criteria for genotypes under water limited conditions. The first morphological effect when potatoes are exposed to drought stress is shown in reduction in leaf size that can affect the canopy architecture (Jefferies and MacKerron 1993). (Ospina 2016) has reported the usefulness of canopy to be used as a selection criterion for nitrogen use efficiency. This study hasshown the relationship between canopy development and nitrogen use efficiency by quantified canopy cover. The genomic region controlling shoot fresh and dry weight under stress conditions were found on chromosome 5. Similarly, Anithakumari et al. (2012) had reported QTL for shoot fresh weight on chromosome 5 under water stress and recovery conditions. QTLs for harvest index dry, total fresh and dry biomass were also identified on chromosome 
5 under drought stress conditions. These findings suggest that the locus on chromosome 5 does not only influence plant maturity but also how genotypes respond to water stress conditions, indicating the effect of maturity on yield under well-watered and drought stress conditions. In our research, late genotypes both under stress and well watered conditions had higher yield that early maturing ones. This suggests that late maturing genotypes can benefit from longer period of photosynthesis that would allow production of more assimilates to be patitioned into tubers. However, under drought stress condition biomass production is tighly linked to transpiration indicating higher yield is associated with increased transpiration efficiency (Blum 2009). Therefore breeding for traits such as root dry mass that increases soil moisture capture for transpiration is important.

The relationships that we have seen between tuber yield and physiological and growth traits under drought stress conditions indicate that there is not one trait that can be used as the drought tolerance indicator; rather it is the aggregated effect of different traits. However, there is a difference in the amount of variation explained by the QTLs linked to these different traits). Moreover, the heritability of a trait is important to consider since response to selection for drought is efficient if traits show correlation to yield and have a high heritability (Blum 2011; Monneveux and Ribaut 2006). Heritability in the current study ranged from moderate to high for several of the traits measured under drought stress conditions. Heritability estimates provide a basis for predicting the response to selection in drought tolerance improvement programs. The higher the heritability estimate, the better the response to selection. Similar heritability ranges for drought tolerance experiments were reported by (Anithakumari et al. 2012; Khan et al. 2015). Since direct selection for tuber yield under drought stress conditions is difficult in most cases, agro-morphological, and physiological traits that have correlation with tuber yield and higher heritability can be used as drought tolerance indicators for an indirect selection.

Drought specific QTLs were detected for tuber yield on chromosome 7, 9, and 12. Also, on chromosome 8 we have found a QTL for plant height specific to stress conditions. These QTLs were expressed only in one experimental year (Table 3; Figure 2). This implies that the expression of the QTLs may be influenced by the difference in environmental conditions (temperature, relative humidity, etc.) between different years (Table 1). The main difference 
between the two experimental years was the minimum temperature recorded, $10.80 \mathrm{C}$ in year 2010 and $4.50 C$ in 2011 . The stability of these QTLs being expressed under water stress conditions might need to be confirmed in a further field trial. However QTL detected on chromosome 12 for tuber fresh weight under drought stress conditions has showed an overlap with QTL detected for onset and inflection point of plant height under normal growing conditions with single trait QTL analysis of Chapter 2 (Hurtado-Lopez et al. 2015). Also, QTL identified on chromosome 8 for plant height specific to drought stress condition has collocated with QTL identified (single trait QTL analysis) on chromosome 8 for a parameter controlling inflection point of senescence under short photoperiod condition (Chapter 2). The QTL detected on chromosome 7 for tuber yield under water stress conditions co-located with a QTL found for tuber number under well-watered conditions indicating same locus control tuber number under both treatment conditions. QTL for tuber number under well-watered conditions was also detected on chromosome 3. Similarly, QTL for tuber number under short photoperiod conditions was reported (Hurtado-Lopez et al. 2015).

A QTL for harvest index based on dry weight was detected on chromosome 5 only under well-watered conditions, Khan et al. (2015) reported QTLs for harvest index based on fresh and dry weight on chromosome 5 for water stress and well-watered conditions. Their harvest index data from fresh weight was collected from both green house and field trials, while data of harvest index from dry weight was collected from green house only. On chromosome 8 a QTL for root dry weight co-located with tuber number and tuber yield under well-watered conditions. Chromosome 8 was reported to contain QTLs for tuber fresh weight, tuber dry weight, harvest index fresh weight under well-watered conditions of a greenhouse experiment (Khan et al. 2015). However, we were not able to compare the colocalization of the QTLs on the same position since different population and markers were used. Nevertheless, this finding indicates that QTL located on chromosome 8 is expressed under different environment (greenhouse and field) and different mapping population. For two of the experimental years QTL for plant height measured under well-watered conditions was detected on chromosome 6, indicating the stablility of the QTL being expressend in different years. 
Chlorophyll content (SPAD measurement) was indicated to have a close correlation with leaf photosynthetic capacity (Kato et al. 2004; Kumagai et al. 2009). Stay green traits are related with maintaining higher chlorophyll content under drought stress conditions and this is associated with higher photosynthetic capacity and better yield (Borrell et al. 2000) In Sorghum, stay green traits are associated with delayed senescence resulting in better yield and biomass under drought stress conditions (Borrell et al. 2001). Therefore maintaining higher chlorophyll content under water stress conditions can help a plant to cope with the effect of stress. In the current study, we have detected 7 QTLs for chlorophyll content on chromosome 2 that were expressed at different time points, 19, 29 and 34 DAS, and in both years, under both drought stress and well-watered conditions. Similarly, Anithakumari et al. (2012) identified a QTL on chromosome 2 for chlorophyll content measured 3 DAS under water stress conditions that overlaps with the QTL positions detected in the current study. This suggests that the QTL present on chromosome 2 for chlorophyll content is a stable QTL that is expressed across treatments, years and environments. Chlorophyll content measured under drought conditions was higher than under well-watered conditions in all the three time points measured (Table 2 ) and this may suggest a possible reduction in leaf size making the leaf appear darker. Increased leaf greenness was reported as a consequence of reduced leaf growth in potato rather than as a delayed senescence under moderate drought stress imposed at onset of tuber initiation (Rolando et al. 2015). However, other reserachers suggest leaf greenness under drought stress conditions can be associated with delayed senescence in potato under drought stress conditions (Yactayo et al. 2013; Ramírez et al. 2014). The different explanation given by these authors about increased chlorophyll content in response to water stress might be linked to the different time and level of stress applied. Therefore, it is important to measure leaf area or score senescence under drought stress conditions in order to confirm chlorophyll content as drought tolerance indicators. In addition, it was reported to consider the developmental stage in which chlorophyll content is measured and the timing and level of stress imposed inorder to consider increased leaf greenness as an indicator of delayed senescence (Rolando et al. 2015). Four other QTLs for chlorophyll content were identified on chromosome 10 for chlorophyll content measured on 19 and 34DAS for both treatment conditions. Chromosome 10 was also reported to contain QTL for chlorophyll content measured on 3 and 8DAS under stress conditions of a greenhouse experiment (Anithakumari et al. 2012), moreover this QTL was reported to co- 
locate with an eQTL belonging to the functional classes of global transcription factors group and putative DNA-binding protein (Anithakumari 2011). Under well-watered conditions, QTL for chlorophyll content co-located with shoot fresh weight and total fresh biomass on chromosome 11, , suggesting a relationship between chlorophyll content and growth traits however the correlation observed between these traits is weak. QTL for chlorophyll content were detected on chromosome 1 under well-watered conditions however it did not colocate with QTL identified for root dry weight under well-watered conditions.

All these QTLs found under drought stress conditions indicate that there is genetic variation in this population that can be used in the improvement program of potato for drought tolerance. Furthermore, studies like these give a better insight in drought tolerance traits that can be used to enhance potato for drought tolerance. However, it is important to confirm stability of drought related QTLs in order to be implemented in marker assisted breeding program. Finding stable QTL for drought tolerance traits, that are expressed across environments can be complex since response to drought is highly influenced by the different developmental stage of potato in which stress is being applied as well as by the severity level of stress imposed. Accounting for different stress scenarios and developmental stages of potato in drought stress experiments could help in finding stable QTLs for drought tolerance traits that can be used to implement in marker assisted selection of potato for enhanced drought tolerance.

\section{Acknowledgments}

We would like to acknowledge Melkassa agricultural research center for the experimental land provision. We would like to thank Berend-Jan Domba for his help during planting the 2010 experiment. We also thank Kindu Gashu for his assistance in planting and phenotyping 2011 experiment. 


\section{References}

Anithakumari A, Dolstra O, Vosman B, Visser RG, van der Linden CG (2011) In vitro screening and QTL analysis for drought tolerance in diploid potato. Euphytica 181 (3):357-369

Anithakumari AM, Nataraja KN, Visser RG, van der Linden CG (2012) Genetic dissection of drought tolerance and recovery potential by quantitative trait locus mapping of a diploid potato population. Molecular breeding : new strategies in plant improvement 30 (3):1413-1429. doi:10.1007/s11032-012-9728-5

Blum A (2009) Effective use of water (EUW) and not water-use efficiency (WUE) is the target of crop yield improvement under drought stress. Field Crops Research 112 (2):119-123

Blum A (2011) Drought resistance-is it really a complex trait? Functional Plant Biology 38 (10):753-757

Borrell A, Hammer G, Oosterom E (2001) Stay-green: A consequence of the balance between supply and demand for nitrogen during grain filling? Ann Appl Biol 138 (1):91-95

Borrell AK, Tao Y, Mclntyre CL (2000) Physiological basis, QTL and MAS of the stay-green drought resistance trait in grain sorghum. Production in Water-Limited Environments:142

Celis-Gamboa BC (2002) The life cycle of the potato (Solanum tuberosum L.): from crop physiology to genetics. PhD Thesis, Wageningen University, Wageningen. ,

Collins A, Milbourne D, Ramsay L, Meyer R, Chatot-Balandras C, Oberhagemann P, De Jong W, Gebhardt C, Bonnel E, Waugh R (1999) QTL for field resistance to late blight in potato are strongly correlated with maturity and vigour. Mol Breeding 5 (5):387-398

Dalla Costa L, Delle Vedove G, Gianquinto G, Giovanardi R, Peressotti A (1997) Yield, water use efficiency and nitrogen uptake in potato: influence of drought stress. Potato Research 40 (1):19-34

Danan S, Veyrieras J-B, Lefebvre V (2011) Construction of a potato consensus map and QTL meta-analysis offer new insights into the genetic architecture of late blight resistance and plant maturity traits. Bmc Plant Biol 11 (1):1

Deblonde P, Haverkort A, Ledent J-F (1999) Responses of early and late potato cultivars to moderate drought conditions: agronomic parameters and carbon isotope discrimination. European Journal of Agronomy 11 (2):91-105

Deblonde P, Ledent J-F (2001) Effects of moderate drought conditions on green leaf number, stem height, leaf length and tuber yield of potato cultivars. European Journal of Agronomy 14 (1):31-41

Gregory P, Simmonds L (1992) Water relations and growth of potatoes. In: The potato crop. Springer, pp 214-246

Henfling JW (1987) Late blight of potato, vol 4. International Potato Center,

Hijmans RJ (2003) The effect of climate change on global potato production. American Journal of Potato Research 80 (4):271-279

Hurtado-Lopez PX, Tessema BB, Schnabel SK, Maliepaard C, Van der Linden CG, Eilers PHC, Jansen J, van Eeuwijk FA, Visser RGF (2015) Understanding the genetic basis of potato development using a multi-trait QTL analysis. Euphytica 204 (1):229-241. doi:10.1007/s10681-015-1431-2

Hurtado PX, Schnabel SK, Zaban A, Veteläinen M, Virtanen E, Eilers PH, Van Eeuwijk FA, Visser RG, Maliepaard C (2012) Dynamics of senescence-related QTLs in potato. Euphytica 183 (3):289-302 
Iwama K (2008) Physiology of the Potato: New Insights into Root System and Repercussions for Crop Management. Potato Research 51 (3-4):333-353. doi:10.1007/s11540-0089120-3

Jefferies R (1995a) Physiology of crop response to drought. In: Potato ecology and modelling of crops under conditions limiting growth. Springer, pp 61-74

Jefferies R, MacKerron D (1993) Responses of potato genotypes to drought. II. Leaf area index, growth and yield. Ann Appl Biol 122 (1):105-112

Jefferies RA (1995b) Physiological determinants of genotypic differences in carbon isotope discrimination in potato grown in well-watered conditions. Ann Appl Biol 127 (3):585592

Kato M, Kobayashi K, Ogiso E, Yokoo M (2004) Photosynthesis and dry-matter production during ripening stage in a female-sterile line of rice. Plant production science 7 (2):184188

Khan MA, Saravia D, Munive S, Lozano F, Farfan E, Eyzaguirre R, Bonierbale M (2015) Multiple QTLs linked to agro-morphological and physiological traits related to drought tolerance in potato. Plant Molecular Biology Reporter 33 (5):1286-1298

Kloosterman B, Abelenda JA, Gomez MdMC, Oortwijn M, de Boer JM, Kowitwanich K, Horvath BM, van Eck HJ, Smaczniak C, Prat S, Visser RGF, Bachem CWB (2013) Naturally occurring allele diversity allows potato cultivation in northern latitudes. Nature 495 (7440):246-250

Kumagai E, Araki A, Kubota $F$ (2009) Correlation of chlorophyll meter readings with gas exchange and chlorophyll fluorescence in flag leaves of rice (Oryza sativa L.) plants. Plant production science 12 (1):50-53

Lahlou O, Ledent J-F (2005) Root mass and depth, stolons and roots formed on stolons in four cultivars of potato under water stress. European Journal of Agronomy 22 (2):159173. doi:10.1016/j.eja.2004.02.004

Lahlou O, Ouattar S, Ledent J-Fo (2003) The effect of drought and cultivar on growth parameters, yield

and yield components of potato. Agronomie 23 (3):257-268. doi:10.1051/agro:2002089

Levy D, Genizi A, Goldman A (1990) Compatibility of potatoes to contrasting seasonal conditions, to high temperatures and to water deficit: The association with time of maturation and yield potential. Potato Research 33 (3):325-334

Monneveux P, Ramirez DA, Pino MT (2013) Drought tolerance in potato (S. tuberosum L.) Can we learn from drought tolerance research in cereals? Plant Science 205:76-86. doi:10.1016/j.plantsci.2013.01.011

Monneveux P, Ribaut J-M (2006) Secondary traits for drought tolerance improvement in cereals. Drought adaptation in cereals:97-143

Muijen D, Anithakumari A, Maliepaard C, Visser RG, Linden CG (2016) Systems genetics reveals key genetic elements of drought induced gene regulation in diploid potato. Plant, Cell \& Environment

Munné-Bosch S, Alegre L (2004) Die and let live: leaf senescence contributes to plant survival under drought stress. Functional Plant Biology 31 (3):203-216

Ospina CA (2016) Nitrogen use efficiency in potato: an integrated agronomic, physiological and genetic approach. PhD Thesis, Wageningen University, Wageningen,

Ramírez D, Yactayo W, Gutiérrez R, Mares V, De Mendiburu F, Posadas A, Quiroz R (2014) Chlorophyll concentration in leaves is an indicator of potato tuber yield in watershortage conditions. Scientia Horticulturae 168:202-209 
Renault D, Wallender W (2000) Nutritional water productivity and diets. Agricultural water management 45 (3):275-296

Rolando JL, Ramírez DA, Yactayo W, Monneveux P, Quiroz R (2015) Leaf greenness as a drought tolerance related trait in potato (Solanum tuberosum L.). Environmental and Experimental Botany 110:27-35

Schafleitner R, Gutierrez R, Espino R, Gaudin A, Pérez J, Martínez M, Domínguez A, Tincopa L, Alvarado C, Numberto G, Bonierbale M (2007) Field Screening for Variation of Drought Tolerance in Solanum tuberosum L. by Agronomical, Physiological and Genetic Analysis. Potato Research 50 (1):71-85. doi:10.1007/s11540-007-9030-9

Schittenhelm S, Sourell H, Löpmeier F-J (2006) Drought resistance of potato cultivars with contrasting canopy architecture. European Journal of Agronomy 24 (3):193-202. doi:10.1016/j.eja.2005.05.004

Tourneux C, Devaux A, Camacho M, Mamani P, Ledent J-F (2003) Effects of water shortage on six potato genotypes in the highlands of Bolivia (I): morphological parameters, growth and yield. Agronomie 23 (2):169-179

Tuberosa R (2012) Phenotyping for drought tolerance of crops in the genomics era. Frontiers in physiology 3:347. doi:10.3389/fphys.2012.00347

Van den Berg J, Ewing E, Plaisted R, McMurry S, Bonierbale M (1996) QTL analysis of potato tuberization. Theoretical and applied genetics 93 (3):307-316

Van Ooijen J (2006) JoinMap 4. Software for the calculation of genetic linkage maps in experimental populations Kyazma BV, Wageningen, Netherlands

Van Ooijen J (2009) MapQTL 6. Software for the mapping of quantitative trait loci in experimental populations of diploid species Kyazma BV: Wageningen, Netherlands

Visker MHPW, Keizer LCP, Van Eck HJ, Jacobsen E, Colon LT, Struik PC (2003) Can the QTL for late blight resistance on potato chromosome 5 be attributed to foliage maturity type? Theoretical and Applied Genetics 106 (2):317-325. doi:DOI 10.1007/s00122-002-1021-2

Voorrips RE (2002) MapChart: Software for the graphical presentation of linkage maps and QTLs. The Journal of Heredity 93 (1):77-78

VSNi (2012) GenStat reference manual. VSN international, Hemel Hempstead 15th edn

Yactayo W, Ramírez DA, Gutiérrez R, Mares V, Posadas A, Quiroz R (2013) Effect of partial root-zone drying irrigation timing on potato tuber yield and water use efficiency. Agricultural Water Management 123:65-70 


\section{Chapter 4 \\ Unraveling the genetic components of drought tolerance of potato grown under moderate water limitation}

Biructawit B Tessema ${ }^{1,2}$, Peter G Vos ${ }^{1,2}$, Ernest Beckee Aliche ${ }^{1,2}$, Daniela Bustos-Korts ${ }^{1,3}$, Richard GF Visser ${ }^{1,2}$, C. Gerard van der Linden ${ }^{1,2}$

${ }^{1}$ Plant Breeding, Wageningen University \& Research, PO Box 386, 6700 AJ Wageningen, the Netherlands

${ }^{2}$ The graduate school of Experimental Plant Sciences (EPS). Wageningen University \& Research, Wageningen, the Netherlands

${ }^{3}$ current address: Biometris, Wageningen University \& Research, P.O. Box 100, 6700 AC Wageningen, The Netherlands 


\begin{abstract}
Unraveling the genetic basis of tolerance to drought in potato is highly desirable for developing potatoes with enhanced drought tolerance. Potatoes are known to be sensitive to even moderate drought stress, causing considerable yield losses. In this study, 82 modern potato cultivars collected from different geographical locations and market segments were used to examine the effect of moderate drought stress under greenhouse conditions. Moderate drought stress started two weeks after emergence by reducing water supply to the plants by $50 \%$ compared to the optimum amount of water. During the stress period, phenotypic evaluations were performed both under water-limited and well-watered conditions. Water limitation resulted in reduced tuber yield and affected growth traits. In order to find the genomic regions contributing to drought tolerance, we applied genome wide association mapping using a 20K SNP array. We detected marker trait associations both under well-watered and water-limiting conditions. Some of the marker traits associations were detected on chromosome 5 and influenced by maturity. However, QTLs for drought tolerance traits were identified on other chromosomes as well (chromosomes 4, 6, 9, 10 and 12) and some of them were drought specific. QTLs for stolonization and tuberization were detected on chromosome 6 and 9, respectively. These QTLs were only found under waterlimited conditions. As a first attempt of applying association mapping in dissecting the genetic basis of drought tolerance, this study gives insight into the genetic architecture of drought tolerance traits in cultivated, tetraploid potato.
\end{abstract}

Keywords: potato, tetraploid, moderate drought stress, association mapping 


\section{Introduction}

Drought stress is one of the most recognized environmental constraints to date for plant survival and crop productivity (Hillel and Rosenzweig 2002; Dai 2011). With climate change, the increasing aridity is an even more important factor threatening agriculture, which is the major spender of sweet water resources in many regions of the world. As the resources such as water and land are further limited, food security in the twenty-first century will rely at least partly on development of improved cultivars with drought resistance and high yield stability (Pennisi 2008; Chapman et al. 2012). Thus, a better understanding of drought stress responses and identification of traits that contribute to drought tolerance is important.

Drought stress is one of the biggest challenges for potato production (Monneveux et al. 2013). Potato (Solanum tuberosum) is the third most important food consumed worldwide and is highly valued as food security crop. However, this crop is sensitive to moderate drought stress causing considerable yield loss (Gregory and Simmonds 1992; Deblonde and Ledent 2001). Potatoes regularly suffer from water shortage in most of their rain fed cultivation regions (Thiele et al. 2010). Furthermore, the impact of drought stress on potato production will most likely increase as changes in climatic conditions are predicted to increase the yield loss in potato by $18-32 \%$ in the coming three decades (Hijmans 2003). The impact of drought stress on potato yield depends on phenological timing, duration and severity of stress (Jefferies 1995). Drought stress affects potato shoot development, leaf expansion, tuber initiation, and tuber yield (Deblonde and Ledent 2001; Anithakumari et al. 2012; Khan et al. 2015).

Improving drought tolerance mainly relies on the existing genetic variation in cultivated potato and the possibility to increase this genetic variation utilizing wild resources. However, the quantitative inheritance and low heritability of drought tolerance has hindered direct selection for yield under drought stress conditions in crops, including potato (Blum 1988; Boyer 1996). Overcoming this limitation is at least partly possible by selecting growth and physiological traits that have higher heritability than yield under water-limited conditions (Ludlow and Muchow 1990). The application of molecular markers enables the dissection of the genetic basis of tolerance to drought with the identification of quantitative trait loci (QTLs) that control drought tolerance traits (Tuberosa and Salvi 2006), which can then be 
combined in breeding for improved drought tolerant potato cultivars (Tuberosa and Salvi 2006).

Compared to drought tolerance studies in other crops like cereals, genetic studies of tolerance to drought stress in potato number only a few (Monneveux et al. 2013). However, some efforts have been made to understand the genetics of drought response and tolerance in diploid potato mapping populations (Anithakumari et al. 2012; Khan et al. 2015). Both studies have identified QTLs for morphological and agronomical drought tolerance traits in a genomic region located on chromosome 5 and these QTLs co-localized with maturity type. Chromosome 5 is known to harbor a major QTL for maturity type (Visker et al. 2003), which is now known to be caused by genetic variation in the CDF1 gene that mediates photoperiodic control of tuberization (Kloosterman et al. 2013). Also, Khan et al. (2015) reported chromosome 8 to carry drought QTLs for agro-morphological traits. However, these studies are limited in resolution of QTL detection since mapping populations are the result of only a single cycle of recombination.

In recent years, genome wide association mapping was shown to be a promising approach for dissecting and understanding the genetic architecture of complex traits. The principle of genome wide association mapping is to associate phenotypic variation with genetic markers in populations of unrelated genotypes by exploiting linkage disequilibrium (LD) between markers and QTLs (Malosetti et al. 2007; Ersoz et al. 2007). Successful application of association mapping for complex traits was demonstrated for amongst others drought tolerance (Xue et al. 2013), salt tolerance (Long et al. 2013), and higher temperature and CO2 (Ingvordsen et al. 2015). The feasibility of association mapping in tetraploid potato was shown by the detection of marker-trait associations for quality traits in potato ( $D^{\prime}$ Hoop et al. 2008). Ospina (2016) reported marker trait associations for physiological and agronomical traits in potato grown under high and low nitrogen input.

In the present study, a 20K potato SNP array (Vos et al. 2015) was used to perform association mapping studies with 82 potato cultivars phenotyped under well-watered and moderate drought stress conditions. This cultivar set was part of a marker-trait association study of quality traits in potato (D'Hoop et al. 2008), and 82 carefully selected cultivars were 
used by Uitdewilligen et al. (2013) for an association mapping study that identified QTLs for maturity type and tuber flesh color. We aimed in this paper at finding QTLs for drought tolerance traits that would contribute to potato yield under water limiting conditions in tetraploid potato cultivars. In addition to several QTLs accumulating on the maturity type locus on chromosome 5, a number of new and promising QTL for traits associated with drought tolerance were identified.

\section{Materials and Methods}

\section{Experimental set up}

A moderate drought stress experiment was conducted under greenhouse conditions between May and August 2012 at Unifarm, Wageningen University \& Research (Wageningen, NL). The greenhouse environmental conditions are presented in Table 1 . In this experiment, 82 potato cultivars were used (Table S1). This core set was selected from a large potato cultivars set used by D'Hoop et al. (2008) and the selection criteria are described in Uitdewilligen et al (2013). The core set included commercial cultivars from different geographical origins, years of release, and market segment to represent as much as possible the genetic variation existing in cultivated potato material. Furthermore, the set included different maturity classes; early, intermediate, and late. The maturity score was used from a previous field experiment conducted under normal growing conditions ( $D^{\prime}$ Hoop et al. 2008). In our study, low and high score represented late and early cultivars respectively.

Potato tubers with uniform sprouts were used as planting material. One tuber was planted per pot containing 5 liters of soil. N-P-K fertilization (Osmocote) was added at planting to ensure nutrient availability. The experiment was arranged in a split plot design with four blocks. The main plot was assigned to treatments (control and drought) and the subplot was assigned to genotypes. There were four replications for drought treatment and the control treatment was replicated three times over the four blocks arranged as an incomplete block design. Genotypes were randomly assigned to the subplots and they were re-arranged within the main plot every two weeks during the growing season to minimize border effects. 
Table 1. Environmental conditions of the greenhouse for the whole experiment period

\begin{tabular}{lcccc}
\hline & May & June & July & August \\
\hline Temperature $\left({ }^{\circ} \mathrm{C}\right)$ & 24.8 & 20.7 & 22.2 & 23.9 \\
Relative humidity (\%) & 63.8 & 75.7 & 69.4 & 65.4 \\
\hline
\end{tabular}

Two weeks after emergence (WAE), a mild drought stress treatment was started by reducing the amount of water applied to four of the drought stress treatment replicates. The supplied amount of water was reduced to $50 \%$ of the optimum watering as monitored by tensiometers in 2 pots per subplot. Soil water content was measured every 30 minutes and drought stressed plants were irrigated when the soil water content dropped below $25 \%$ as evaluated with a Grodan water content meter (\%vol/vol) and were kept at $25 \%$. Controls received optimal amount of water throughout the experiment.

\section{Phenotyping}

Phenotyping started one week after planting by scoring plant emergence. Chlorophyll content (CC) was measured using a SPAD 502 chlorophyll meter (Minolta Co., Ltd Japan) at three different time points: 16,36 , and 49 days after starting the stress treatment (DAS). Upper young fully opened (sink; Y) and middle (source; M) leaflets were tagged and the same leaflets were measured for two of the time points, 36 (CC36DAS) and 49DAS (CC49DAS), while only upper young fully opened leaflets were measured on 16 DAS (CC16DAS). The area of the third single leaf from the top (in $\mathrm{cm} 2$ ) was measured 30DAS by taking a picture at a fixed distance and angle with a contrasting blue background. The blue background was removed with image J 1.47 (Schneider et al. 2012) and the area was calculated by counting the number of pixels covered by leaflets.

Time taken for the first stolon to appear was checked visually twice a week; from here on we refer to this trait as stolonization. Similarly, appearance of the first tuber bigger than $1 \mathrm{~cm}$ in diameter was recorded as the time of tuberization. Both traits were recorded in weeks after emergence. Plant height $(\mathrm{cm})$ was measured by stretching the longest stem on the 30th day of water stress. At the end harvest 97 days after planting (77 DAS) fresh tuber yield (g) was recorded. Shoot dry weight was measured after drying at a temperature of $70 \circ \mathrm{C}$ until 
constant weight was reached. Root and stolon dry weight was measured together as one trait.

\section{Statistical analysis}

Statistical analysis was performed using Genstat 15th edition (VSN, international Ltd., Oxford, UK). Descriptive statistics for each trait under both stress and control conditions were calculated. Adjusted means were calculated for each genotype and water treatment using the Equation 1 that accounts for the experimental design factors;

$$
\mathrm{y}_{\mathrm{ijk}}=\mu+\rho_{\mathrm{k}}+\alpha_{\mathrm{i}}+\underline{\mathrm{d}}_{\mathrm{ik}}+\beta_{\mathrm{j}}+(\alpha \beta)_{\mathrm{ij}}+\underline{\mathrm{e}}_{\mathrm{ijk}}
$$

Where $y_{i j k}$ is the mean of genotype $i$ in water treatment $j, \mu$ is the general mean, $\rho_{k}$ is the fixed block effect, $\alpha_{\mathrm{i}}$ is the fixed effect of the whole plot water treatment (drought and control treatments), $\underline{d}_{i k}$ is a random term that represents whole plot error, $\beta_{j}$ is the fixed effect of the sub plot treatment (genotype), $(\alpha \beta)_{\mathrm{ij}}$ represents the interaction effects between water treatment and genotype (fixed). $\underline{e}_{i j k} \sim N\left(0, \sigma_{e}^{2}\right)$ is the residual variation.

Broad sense heritability $(\mathrm{H} 2)$ was calculated according to the formula $\mathrm{H}^{2}=\sigma_{\mathrm{g}}^{2} /\left(\sigma_{\mathrm{g}}^{2}+\frac{\sigma_{\mathrm{e}}^{2}}{\mathrm{r}}\right)$, where $\sigma_{\mathrm{g}}^{2}$ is genetic variance, $\sigma_{\mathrm{e}}^{2}$ is environmental variance, and $\mathrm{r}$ is number of replications ( 3 for control and 4 for water stress). Pearson correlation coefficients were calculated for all traits measured for both drought and control treatments.

\section{Association mapping}

Association mapping analysis was performed with the 82 cultivars using a 20K Infinium SNP array (Vos et al. 2015). Briefly, the 20K SNP array contains 15,138 SNPs identified in a previous study (Uitdewilligen et al. 2013) and 4454 SNPs from the Sol-CAP project (Hamilton et al. 2011). The results from the SNP array were analyzed with the software program fitTetra (Voorrips et al. 2011) in five SNP dosage classes. The dosage classes are nulliplex, simplex, duplex, triplex and quadruplex depending on the number of allele copies (0 to 4). SNPs with allele frequencies higher than $5 \%$ in at least two of the dosage classes were considered for the analysis. 
Association analysis was done after correcting for population structure using a kinship matrix. The kinship matrix was calculated using 764 markers that were randomly distributed over the genome. A mixed model was used to find associations between marker and traits (Equation 2). This model takes into account genetic relatedness and uses the kinship matrix to correct for it.

The mixed model was:

$$
\mathrm{y}_{\mathrm{i}}=\mu+\mathrm{x}_{\mathrm{iq}} \alpha_{\mathrm{q}}+\underline{\mathrm{G}}_{\mathrm{i}}+\mathrm{e}_{\mathrm{i}}
$$

Where $y_{i}$ is the phenotype if genotype $i, \mu$ is a fixed intercept term, $x_{i q}$ is a genotypic covariable that represents DNA information of genotype $i$ at QTL position $q$, and $\alpha_{q}$ is the additive effect of the fixed QTL q. $\underline{G}_{i}$ is a random term that accounts for population structure ,. $\underline{G}_{\mathrm{i}} \sim \mathrm{N}\left(0, \mathrm{~K} \sigma_{\mathrm{g}}^{2}\right)$, where $\mathrm{K}$ represents the Kinship matrix.

\section{Results}

\section{Treatment effect}

The 82 cultivars used in the present study showed highly significant phenotypic variation in response to treatment, even though the drought stress applied was only moderate (Table 2). There were significant differences between well-watered and water-limited conditions for all measured traits. Genotypic differences showed highly significant variations for all the traits as well. The interaction term between genotype and treatment showed significant variation for most traits, except for stolonization, CC16DASY, and CC49DASM. 
Table 2. Analysis of variance for the list traits measured with their treatment and genotype effect and their interactions.

\begin{tabular}{lccc}
\hline & \multicolumn{3}{c}{ P value } \\
\cline { 2 - 4 } Traits & $\begin{array}{c}\text { Treatment } \\
(\mathrm{T})\end{array}$ & $\begin{array}{c}\text { Genotype } \\
(\mathrm{G})\end{array}$ & GxT \\
\hline \hline Plant height $(\mathrm{cm})$ & 0.007 & $<0.001$ & 0.001 \\
Shoot Dry weight (g) & 0.018 & $<0.001$ & $<0.001$ \\
Root-stolon dry weight & & & \\
(g) & 0.018 & $<0.001$ & $<0.001$ \\
Single Leaf area & 0.001 & $<0.001$ & $<0.001$ \\
Stolonization (WAE) & 0.045 & $<0.001$ & 0.200 \\
Tuberization (WAE) & 0.033 & $<0.001$ & 0.005 \\
Tuber yield (g) & 0.011 & $<0.001$ & $<0.001$ \\
CC16DASY & $<0.001$ & $<0.001$ & 0.241 \\
CC36DASY & $<0.001$ & $<0.001$ & $<0.001$ \\
CC36DASM & 0.058 & $<0.001$ & $<0.001$ \\
CC49DASY & 0.015 & $<0.001$ & 0.031 \\
CC49DASM & 0.044 & $<0.001$ & 0.777 \\
\hline
\end{tabular}

$\mathrm{CC}=$ Chlorophyll content, $\mathrm{DAS}=$ days after stress, $\mathrm{Y}=$ Young leaflet, $\mathrm{M}=$ middle leaflet

\section{Reduction due to drought stress}

Relative reduction was computed as the mean difference between control and drought stress treatment divided by control mean for each genotype and was converted into percentages (Figure 1). Water limitation resulted in a reduction for tuber yield and above ground traits, which included plant height, shoot dry weight and single leaf area. Some of the traits including root stolon dry weight and chlorophyll content had higher values under water stress conditions hence a negative value for relative reduction (Figure 1). In addition, stolonization and tuberization were delayed under water limited conditions resulting in a negative value for relative reduction (Figure 1). The relative differences in measured traits due to water shortage seemed to be influenced by maturity class. Tuber yield showed a strong reduction in response to water stress and the strongest reduction was seen for late maturing genotypes; however the differences among maturity groups were not significant. Late maturing genotypes had a higher reduction for shoot dry weight and single leaf area compared to early and intermediate maturity types. This variation between maturity classes was also observed for tuber initiation and stolonization. Delays in stolonization and 
tuberization were more pronounced for intermediate genotypes than early and late genotypes under water limiting conditions. Root-stolon dry weight was higher for intermediate genotypes followed by late genotypes under water stress conditions. The relative differences for chlorophyll content indicated higher densities of leaf chlorophyll under water-limited conditions, which may indicate that plants had lower leaf expansion rates under stress.

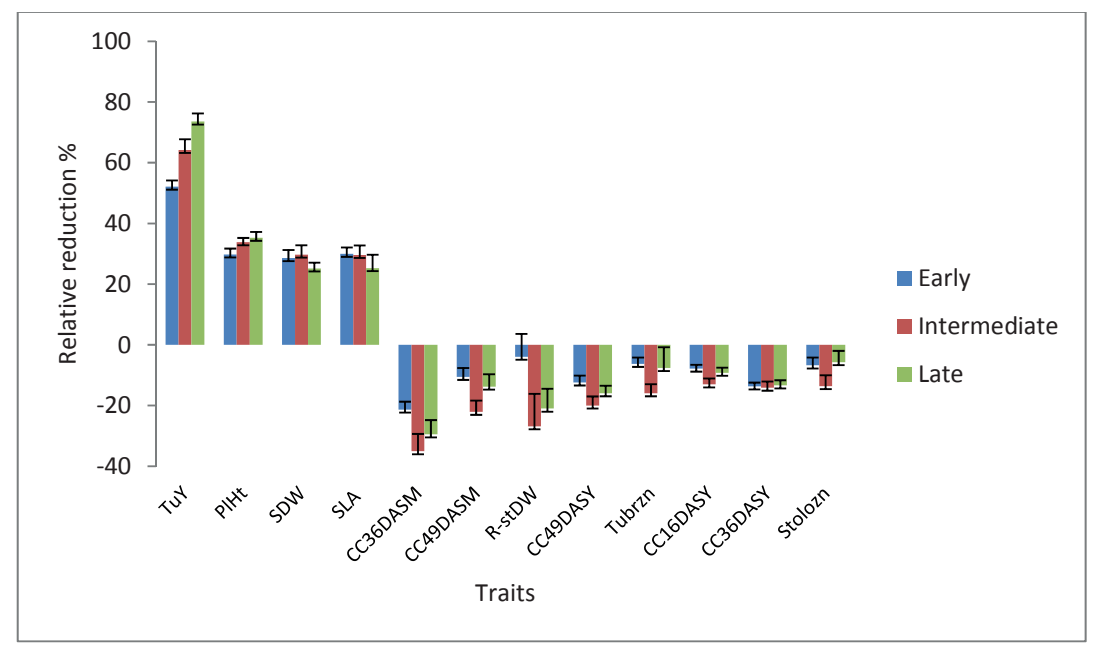

Figure 1. Relative differences of traits grouped based on their maturity class. TuY= Tuber yield, PIHt= Plant height, SDW= shoot dry weight, SLA= Single leaf area, R-stDW= Root-stolon dry weight, Tubrzn= Tuberization, Stolozn= Stolonization. $\mathrm{CC}=$ Chlorophyll content, $\mathrm{DAS}=$ days after stress, $\mathrm{Y}$ and $\mathrm{M}$ represents young and middle leaflets used for measuring chlorophyll content. Error bar indicates the standard error of the mean of relative reduction.

\section{Correlation and heritability of traits}

In the correlation analysis of traits, maturity type was shown to be significantly correlated to most of the growth traits under both treatment conditions (Figure 2). Under both treatment conditions, maturity type had a highly significant $(P<0.001)$ positive correlation with tuber yield, indicating that early genotypes had higher tuber yield. This is in line with previous findings of drought evaluation of a diploid potato mapping population in pots in the 
greenhouse (Anithakumari et al. 2012) but contrary to what was found for field conditions; late maturing genotypes typically produced higher tuber yield than early ones in the field (Deblonde and Ledent 2001; Chapter 3 of this thesis). The below-ground trait root-stolon development had a highly significant negative correlation $(-0.81)$ with tuber yield as well as with maturity $(-0.77)$, indicating that late genotypes with higher investment in roots and stolons did not benefit in terms of yield. A similar result was reported by Tourneux et al. (2003), where late genotypes with higher root dry mass had lower tuber yield than early genotypes in a pot experiment done in greenhouse. Maturity had a negative correlation with shoot dry weight under both treatment conditions, indicating late genotypes had higher shoot dry weight. This is in agreement with what was found by Anithakumari et al. (2012). Under water-limited conditions, tuber yield is much more negatively correlated with shoot dry weight than under well-watered conditions (Figure 2), indicating that a well-developed foliage under water-limiting conditions at the time of harvest may present a disadvantage in our experiments. Most traits showed high correlations between water-limited and wellwatered conditions (Figure 2, diagonal).

\begin{tabular}{|c|c|c|c|c|c|c|c|c|c|c|c|c|c|c|}
\hline & & 1 & 2 & 3 & 4 & 5 & 6 & 7 & 8 & 9 & 10 & 11 & 12 & 13 \\
\hline Maturity & 1 & 1.00 & -0.73 & 0.51 & -0.47 & -0.69 & -0.26 & 0.26 & -0.21 & -0.22 & -0.10 & 0.02 & -0.11 & -0.17 \\
\hline Root-stolon dry weight & 2 & -0.77 & 0.59 & -0.37 & 0.49 & 0.56 & 0.44 & -0.31 & 0.19 & 0.16 & 0.01 & -0.01 & 0.18 & 0.14 \\
\hline Tuber yield & 3 & 0.77 & -0.80 & 0.62 & -0.19 & -0.39 & -0.51 & 0.13 & -0.39 & -0.19 & -0.08 & -0.11 & -0.15 & -0.19 \\
\hline shoot dry weight & 4 & -0.61 & 0.70 & -0.73 & 0.77 & 0.61 & 0.18 & -0.34 & 0.22 & 0.03 & -0.09 & -0.15 & -0.04 & -0.03 \\
\hline Plant height & 5 & -0.45 & 0.50 & -0.54 & 0.65 & 0.69 & 0.25 & -0.10 & 0.13 & 0.28 & 0.15 & -0.11 & 0.19 & 0.01 \\
\hline Tuberization & 6 & -0.22 & 0.35 & -0.38 & 0.41 & 0.19 & 0.38 & 0.03 & 0.53 & 0.29 & 0.14 & 0.10 & 0.39 & 0.21 \\
\hline single leaf area & 7 & 0.23 & -0.18 & 0.27 & -0.24 & -0.06 & -0.01 & 0.62 & -0.12 & -0.05 & 0.07 & 0.11 & 0.04 & -0.10 \\
\hline Stolonization & 8 & -0.21 & 0.22 & -0.41 & 0.32 & 0.12 & 0.46 & -0.09 & 0.62 & 0.15 & -0.01 & 0.19 & 0.36 & 0.34 \\
\hline CC16DASY & 9 & -0.34 & 0.19 & -0.30 & 0.22 & 0.34 & 0.09 & -0.05 & 0.24 & 0.63 & 0.66 & 0.38 & 0.60 & 0.52 \\
\hline CC36DASY & 10 & -0.12 & 0.09 & -0.05 & -0.01 & 0.22 & -0.11 & 0.04 & 0.01 & 0.53 & 0.59 & 0.44 & 0.40 & 0.38 \\
\hline CC36DASM & 11 & -0.17 & 0.04 & -0.05 & -0.07 & -0.10 & 0.08 & 0.01 & 0.13 & 0.33 & 0.34 & 0.29 & 0.47 & 0.73 \\
\hline CC49DASY & 12 & -0.32 & 0.19 & -0.38 & 0.33 & 0.50 & 0.06 & -0.20 & 0.31 & 0.65 & 0.45 & 0.21 & 0.52 & 0.65 \\
\hline CC49DASM & 13 & -0.33 & 0.24 & -0.33 & 0.24 & 0.33 & 0.15 & -0.05 & 0.35 & 0.46 & 0.36 & 0.30 & 0.62 & 0.41 \\
\hline
\end{tabular}

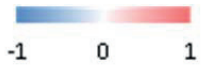

Figure 2. Heat map showing Pearson correlations among traits measured under both wellwatered and water-limited conditions. The upper right triangle is for control and the lower 
left triangle is for moderate drought stress conditions. For trait acronyms see materials and methods section.

For most of the traits, heritability ranged from high to very high (0.64 to 0.93 ) under both well-watered and moderate drought stress conditions (Table 3). The higher heritability value for most of the traits indicates that phenotypic variation has a strong genetic component. We observed small differences in heritability value under control and stress treatments for several of the traits. Heritability for tuber yield was 0.82 and 0.88 under control and water limited conditions, respectively. The highest heritability (0.93) was observed for the below ground trait root-stolon dry weight and chlorophyll content measured on young leaflets at 36 days after stress under water-limited conditions. Shoot dry weight showed high heritability under water-limited conditions as well, with a heritability value of 0.92 and 0.84 in control conditions. Plant height had a heritability value of 0.79 and 0.81 under wellwatered and stress conditions respectively. Under stress conditions, stolonization and tuberization had a heritability value of 0.64 and 0.75 , respectively. Moderate heritable value was observed for chlorophyll content measured on middle leaflet at 49 days after stress for both control and stress conditions. 
Table 3. Descriptive statistics for phenotypic traits measured under well-watered (WW) and water-limited (WL) conditions with their P value from analysis of variance, and broad sense heritability ( $\mathrm{H} 2)$.

\begin{tabular}{|c|c|c|c|c|c|c|}
\hline & \multirow[b]{2}{*}{ Treatment } & \multirow[b]{2}{*}{ Mean } & \multicolumn{2}{|c|}{ Range } & \multirow[b]{2}{*}{ Pvalue } & \multirow[b]{2}{*}{$H^{2}$} \\
\hline & & & Minimum & Maximum & & \\
\hline \multirow[t]{2}{*}{ Plant height (cm) } & WW & 144.0 & 74.3 & 197.3 & $<0.001$ & 0.79 \\
\hline & WL & 96.0 & 48.5 & 138.2 & $<0.001$ & 0.81 \\
\hline \multirow[t]{2}{*}{ Shoot dry weight (g) } & WW & 22.5 & 9.7 & 39.9 & $<0.001$ & 0.84 \\
\hline & $W L$ & 15.8 & 8.8 & 26.7 & $<0.001$ & 0.92 \\
\hline \multirow[t]{2}{*}{$\begin{array}{l}\text { Root-stolon dry } \\
\text { weight }(\mathrm{g})\end{array}$} & WW & 3.5 & 0.5 & 12.2 & $<0.001$ & 0.92 \\
\hline & WL & 4.1 & 0.4 & 11.1 & $<0.001$ & 0.93 \\
\hline \multirow[t]{2}{*}{ Single Leaf area } & WW & 114.4 & 50.0 & 206.1 & $<0.001$ & 0.61 \\
\hline & WL & 79.7 & 42.7 & 119.2 & $<0.001$ & 0.79 \\
\hline \multirow[t]{2}{*}{ Stolonization (WAE) } & WW & 4.5 & 3.0 & 7.0 & $<0.001$ & 0.59 \\
\hline & WL & 4.8 & 3.4 & 6.5 & $<0.001$ & 0.64 \\
\hline \multirow[t]{2}{*}{ Tuberization (WAE) } & WW & 5.4 & 4.0 & 7.5 & $<0.001$ & 0.69 \\
\hline & WL & 5.9 & 4.5 & 8.3 & $<0.001$ & 0.75 \\
\hline \multirow[t]{2}{*}{ Tuber yield (g) } & WW & 262.3 & 56.7 & 480.7 & $<0.001$ & 0.82 \\
\hline & WL & 102.9 & 0.0 & 200.9 & $<0.001$ & 0.88 \\
\hline \multirow[t]{2}{*}{ CC16DAS } & WW & 40.8 & 32.2 & 53.2 & $<0.001$ & 0.64 \\
\hline & WL & 44.6 & 34.8 & 57.0 & $<0.001$ & 0.67 \\
\hline \multirow[t]{2}{*}{ CC36DASY } & WW & 38.5 & 30.6 & 50.1 & $<0.001$ & 0.90 \\
\hline & WL & 43.7 & 35.2 & 53.6 & $<0.001$ & 0.93 \\
\hline \multirow[t]{2}{*}{ CC36DASM } & WW & 29.2 & 17.2 & 38.0 & $<0.001$ & 0.93 \\
\hline & WL & 36.5 & 29.6 & 46.3 & $<0.001$ & 0.83 \\
\hline \multirow[t]{2}{*}{ CC49DASY } & WW & 34.0 & 23.8 & 42.8 & $<0.001$ & 0.59 \\
\hline & WL & 39.1 & 28.1 & 51.9 & $<0.001$ & 0.71 \\
\hline \multirow[t]{2}{*}{ CC49DASM } & WW & 25.8 & 17.4 & 32.8 & $<0.001$ & 0.35 \\
\hline & WL & 29.5 & 20.1 & 40.0 & $<0.001$ & 0.57 \\
\hline
\end{tabular}

$\mathrm{WAE}=$ weeks after emergence 


\section{Population structure}

The phenotypic variations observed for the different agronomical and morphological data were influenced by the maturity type. Principal coordinate analysis (PCO) was used to assess whether there were main determinants for the phenotypic variation present in the set of cultivars using sub set of marker data and maturity score (Figure 3). The first axis of the analysis explained only $6.74 \%$ while the second axis explained $4.43 \%$ of the genotypic variation, indicating that structure of this population of genotypes was low. Maturity type, as one of the main determining factors for yield and growth traits, was not a major driver for population structure. The majority of early genotypes overlapped with intermediate and late genotypes (Figure 3) and a small cluster was observed with few genotypes from intermediate and late genotypes.

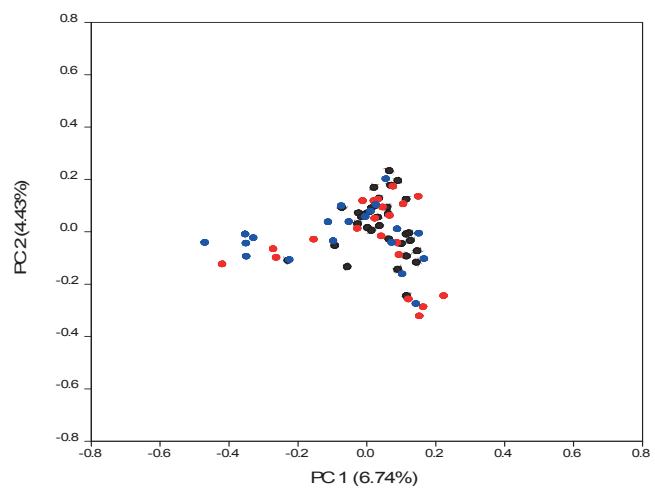

Figure 3. Principal coordinate analysis of 82 cultivars. Symbols of different colors represent different maturity classes (black= early, red= intermediate, and blue= late)

\section{Association mapping}

Association mapping was performed after applying correction for relatedness among the potato cultivars used in the present study. Significant marker trait associations with a value of $-\log 10(p)>4$ are reported. Significant marker trait associations were detected for most of the traits measured under well-watered and water-limited conditions. Under wellwatered conditions there were 22 SNPs significantly associated with eight different traits while under water-limited conditions 37 significant SNPs were associated with ten traits 
(Supplementary file; Table S2 and S3). From the traits that showed significant marker trait associations under water limiting conditions, Manhattan plots for few of them are shown in figure 4.

The most significant markers associated with traits measured under well-watered and waterlimited conditions explaining more than $10 \%$ of the observed phenotypic variation are presented in Table 4. Several significant marker trait associations co-localized with the known maturity locus on chromosome 5 (Visker et al. 2003; Anithakumari et al. 2012; Kloosterman et al. 2013), including tuber yield, plant height, shoot dry weight, root-stolon dry weight and tuberization, under water-limited conditions. Marker trait associations under water stress were detected on several other loci as well, including loci on chromosome 6 and 9 for stolonization and tuberization, respectively.

A marker associated with tuber yield was found on chromosome 5 that explained $16 \%$ of the phenotypic variation under stress conditions. For control conditions, a marker associated with tuber yield was detected on chromosome 10 explaining $23 \%$ of phenotypic variation. For plant height under water stress conditions, significant marker traits associations were identified on chromosome 4 and 5 that respectively explained 17 and $21 \%$ of phenotypic variation. While Under control conditions, significant marker trait associations were identified on chromosome $4,8,11$, and 12 , each explained $16,23,22$, and $10 \%$ of the observed variance respectively. Significant marker trait associations for tuberization were detected on chromosome 5 and 9 specifically for water-limited conditions, explaining 19 and $25 \%$ of phenotypic variation respectively. Drought delayed tuber formation was especially clear for intermediate genotypes, and the strongest marker association for this trait is present on chromosome 9 rather than on the maturity locus on chromosome 5. A marker significantly associated with stolonization specific to stress conditions was detected on chromosome 6 and explained 20\% of the variation. 

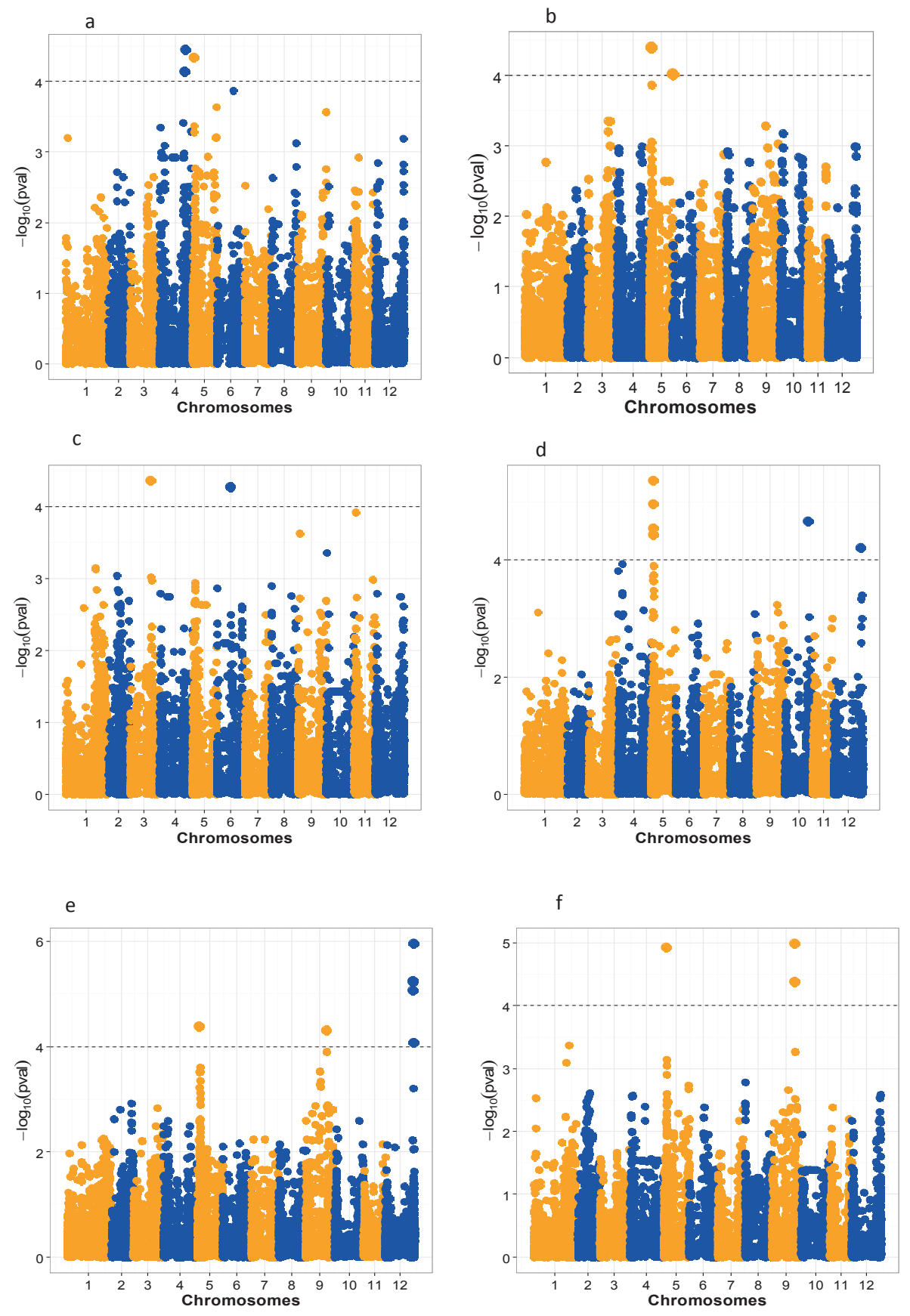

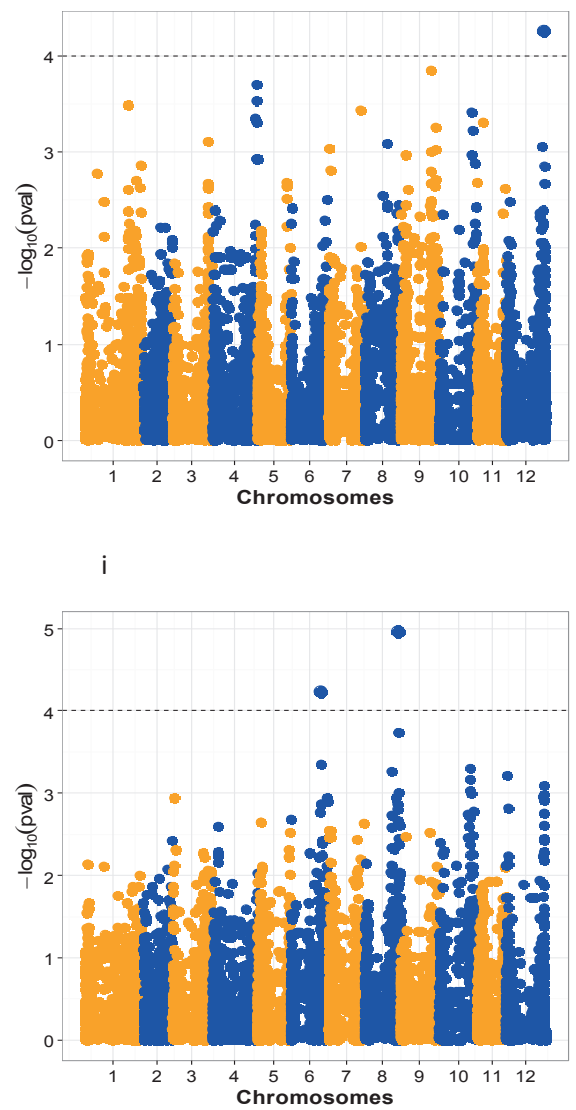

h

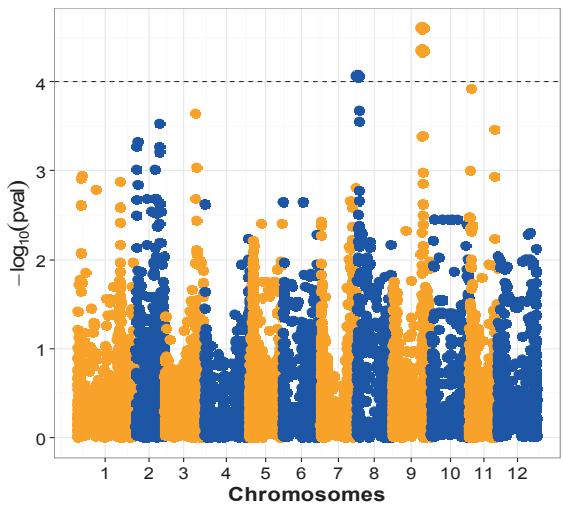

j

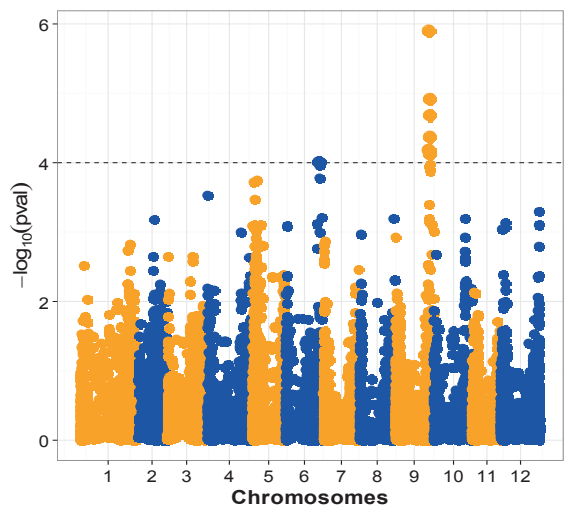

Figure 4 Mahnhattan plots resulting from genome wide association mapping for the traits measured under water-limited conditions, (a) plant height, (b) tuber yield, (c) stolonization, (d) rootstolonshoot dry weight, (e) shoot dry weight, (f) tuberization, (g) CC36DASY, (h) CC36DASM, (i) CC49DASM, (j) CC49DASY. Dotted horizontal line represents the threshold level of $-\log _{10}$ (Pval) of 4 . For abbreviations refer main text. 
The underground trait root-stolon dry weight had significant associations with markers present on chromosomes 5, 10 and 12 under water stress conditions (Table 4). The strongest marker association with root-stolon dry weight that explained $36 \%$ of phenotypic variance was present on chromosome 5 close to the maturity locus. The significant markers present on chromosome 10 and 12 explained moderate amounts of observed variance, 19 and $13 \%$ respectively, while under well-watered conditions, marker trait associations for root-stolon dry weight were detected on chromosome 3, 5, and 6 explaining 17, 30 and 22\% of observed variations respectively. Significant marker trait associations detected for shoot dry weight were located on chromosome 5, 9, and 12 under water stress conditions, explaining respectively 25,18 , and $17 \%$ of the variation.

Markers associated with chlorophyll content measured at 36 (CC36DASY and CC36DASM) days after stress were detected on chromosomes 12, 8 and 9 under water-limited conditions. Markers on chromosome 8 and 9 were associated to chlorophyll content measured on middle leaves and the strongest marker association for this trait was located on chromosome 9, which explained $20 \%$ of observed variance. Marker trait association detected for CC36DASY on chromosome 9 was very close to or on the same locus as the marker-trait association for tuberization (Table 4). Significant marker trait associations for chlorophyll content measured on 49 DAS for both young and middle leaflets were identified on chromosomes 6 and 9. The strongest associations for both traits were located on chromosome 6 and explained 12\% and 16\% of observed variance for young and middle leaf respectively. For well-watered conditions, marker trait associations were found for chlorophyll content (CC16DASY, CC36DASY, and CC49DASM) on chromosome 1, 2, 10, 11, and 12 (Table 4) while for single leaf area a significant marker trait association was located on chromosome 4. 

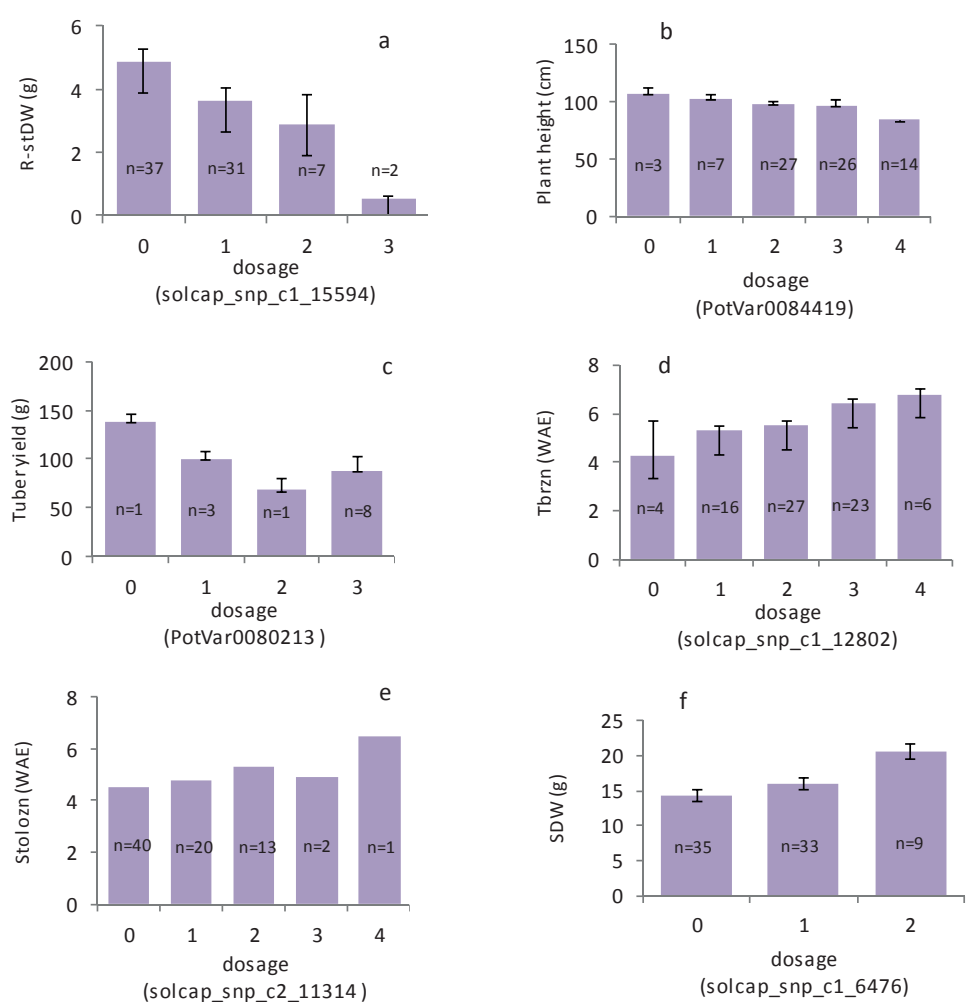

Figure 5. SNP dosage effect on traits measured under water-limited conditions. The $x$-axis shows SNP marker and dosage in 5 classes; $0=$ nulliplex, $1=$ simplex, $2=$ duplex, $3=$ triplex, and 4= quadruplex. SDW= shoot dry weight, Tbrzn= tuberization, stolozn=stolonization and R$s t D W=$ root stolon dry weight. Error bar indicates the standard error of the mean of traits in each dosage class.

The dosage effect of the most significant peak markers associated with the traits measured under water-limited conditions is presented in Figure 5, showing how trait average values varies depended on the allele dosage. We observe a correlation between allele dosages of Solcap_snp_c1_15594 present on chromosome 10 with the mean value of root-stolon dry weight (Figure 5a). The absence of this allele in the cultivars resulted in a significantly higher 
mean value of root-stolon dry weight, where as its presence in triplex dose resulted in a lower mean value. However, there are only two cultivars with triplex dosage, and none with quadruplex dosage. A decrease in plant height was seen with an increase in allele dosage of the marker (PotVar0084419) located on chromosome 4 (Figure 5b). Similarly, we see a decrease in mean tuber yield when allele (PotVar0080213) dosage increased from nulliplex to duplex but this trend does not continue for triplex. As the allele dosage for solcap_snp_c1_12802 on chromosome 9 increased from nulliplex to quadruplex, we observed a delay in tuberization. A delay in stolon appearance was seen as the dosage of allele (solcap_snp_c2_11314) increased, except for triplex (Figure 5e). An increase in shoot dry weight measured under water-limited condition was seen from nulliplex to duplex allele dosage (solcap_snp_c1_6476). Higher dosages for this marker were not observed in our cultivar set. 
Table 4. The most significant marker trait associations detected under well-watered (WW) and water-limited (WL) conditions. The location of the markers with their respective -log 10(P), allele frequency and effect, and phenotypic variance explained (\%) are indicated.

\begin{tabular}{|c|c|c|c|c|c|c|}
\hline Treatment & Trait & Chromosome & $\begin{array}{l}\text { Genome } \\
\text { position }\end{array}$ & Marker name & $-\log _{10}(P)$ & $\begin{array}{l}\text { Variance } \\
\text { explained }\end{array}$ \\
\hline \multirow{14}{*}{ Well-watered } & Tuber yield & 10 & 707277375 & PotVar0122848 & 4.16 & 23 \\
\hline & \multirow[t]{4}{*}{ Plant height } & 4 & 283407138 & PotVar0116182 & 4.12 & 16 \\
\hline & & 8 & 510726488 & solcap_snp_c1_9785 & 4.13 & 23 \\
\hline & & 11 & 726858998 & PotVar0060023 & 4.31 & 22 \\
\hline & & 12 & 776351708 & PotVar0069306 & 4.05 & 10 \\
\hline & \multirow[t]{3}{*}{ Root stolon dry weight } & 3 & 159370115 & PotVar0019246 & 4.01 & 17 \\
\hline & & 5 & 316611906 & solcap_snp_c2_50302 & 5.23 & 30 \\
\hline & & 6 & 419961527 & PotVar0070124 & 4.51 & 22 \\
\hline & Leaf area & 4 & 291081071 & PotVar0099073 & 4.59 & 20 \\
\hline & CC16DASY & 1 & 46273159 & PotVar0132293 & 4.48 & 13 \\
\hline & \multirow[t]{3}{*}{ CC36DASY } & 10 & 652427095 & PotVar0108085 & 4.27 & 21 \\
\hline & & 11 & 717880690 & PotVar0064142 & 4.44 & 24 \\
\hline & & 12 & 827167202 & solcap_snp_c2_39393 & 4.43 & 20 \\
\hline & CC49DASM & 2 & 139930088 & solcap_snp_c2_7559 & 4.79 & 21 \\
\hline \multirow{20}{*}{ Water-limited } & Tuber yield & 5 & 316278656 & PotVar0080213 & 4.40 & 16 \\
\hline & \multirow[t]{2}{*}{ Plant height } & 4 & 287670623 & PotVar0084419 & 4.44 & 17 \\
\hline & & 5 & 315369759 & PotVar0025609 & 4.33 & 21 \\
\hline & \multirow[t]{4}{*}{ Shoot dry weight } & 5 & 315369759 & PotVar0025609 & 4.38 & 25 \\
\hline & & 9 & 620329173 & solcap_snp_c1_6476 & 4.31 & 18 \\
\hline & & 12 & 831892141 & PotVar0053356 & 5.25 & 17 \\
\hline & & 12 & 832115480 & PotVar0053166 & 5.95 & 17 \\
\hline & \multirow[t]{3}{*}{ Root-Stolon dry weight } & 5 & 315963223 & PotVar0078045 & 4.95 & 36 \\
\hline & & 10 & 697993735 & solcap_snp_c1_15594 & 4.67 & 19 \\
\hline & & 12 & 830509804 & PotVar0018338 & 4.20 & 13 \\
\hline & Stolonization & 6 & 403936989 & solcap_snp_c2_11314 & 4.27 & 20 \\
\hline & \multirow[t]{2}{*}{ Tuberization } & 5 & 316278656 & PotVar0080213 & 4.92 & 19 \\
\hline & & 9 & 623998221 & solcap_snp_c1_12802 & 4.98 & 25 \\
\hline & CC36DASY & 12 & 833614485 & solcap_snp_c2_5474 & 4.25 & 15 \\
\hline & \multirow[t]{2}{*}{ CC36DASM } & 8 & 513405102 & PotVar0108992 & 4.06 & 10 \\
\hline & & 9 & 623998221 & solcap_snp_c1_12802 & 4.35 & 20 \\
\hline & \multirow[t]{2}{*}{ CC49DASY } & 6 & 427408071 & PotVar0040034 & 4.00 & 12 \\
\hline & & 9 & 631301143 & solcap_snp_c2_46777 & 4.12 & 12 \\
\hline & \multirow[t]{2}{*}{ CC49DASM } & 8 & 564384310 & solcap_snp_c2_16997 & 4.95 & 16 \\
\hline & & 6 & 421984583 & PotVar0090783 & 4.23 & 15 \\
\hline
\end{tabular}




\section{Discussion}

Several authors have reported on the effects of drought on potato (Deblonde and Ledent 2001; Lahlou et al. 2003; lerna and Mauromicale, 2006; Schafleitner 2007; Anithakumari et al. 2012; Khan et al. 2015). It is known that potato yield is already affected by moderate water limitation (Gregory and Simmonds 1992; Deblonde and Ledent 2001). Enhancing potato yield under water-limited conditions is therefore an important breeding goal. However, breeding for drought tolerance can be difficult since many genes are involved in controlling drought responses in potato (Anithakumari et al. 2012; Khan et al. 2015). This complex nature of drought tolerance poses a challenge for direct selection of yield under water stress conditions. Thus, drought tolerance traits with higher heritability can be used as an indirect selection for yield under drought stress conditions (Blum 2011; Anithakumari et al. 2012). For this reason, dissecting drought tolerance traits that contribute to yield is important.

Recently, several studies have been done to dissect the genetic basis of drought tolerance in potato (Anithakumari et al. 2011; Anithakumari et al. 2012; Khan et al. 2015; Chapter 3 of this thesis). We have summarized the QTLs found in these studies and in this chapter in Table 5. Thus far, drought tolerance studies in potato were done on segregating diploid mapping populations using linkage map based approach. These studies have identified multiple QTLs for agro-morphological and physiological drought tolerance traits. Anithakumari et al. (2012) have reported many drought related QTLs co-localizing with maturity QTL on chromosome 5 in drought tolerance experiment done under controlled greenhouse. Chromosome 5 was shown to be important for drought tolerance in an experiment done under field condition using CxE diploid potatoes (Chapter 3 of this thesis). In addition to a QTL region on chromosome 5, a region with drought tolerance QTLs on chromosome 8 was reported in a drought tolerance experiment done both under greenhouse and field conditions (Khan et al. 2015). However, it is important to consider that the time and amount of stress application influences drought response in potato (Jefferies 1995). These studies have applied severe stress at different developmental stages for a different period of time, which may have an effect on the QTLs that can be detected. 
Here we present an association mapping done in a set of tetraploid cultivars to find the genetic components responsible for stress tolerance under moderate drought stress conditions. The findings from the present study complement previous drought tolerance studies for a better understanding of drought response in potato under different kinds of stress conditions. Although statistical support for the QTLs may be limited because of the relatively low number of genotypes, the 82 genotypes were chosen to have little or no population structure while representing wide diversity, and in a previous study a similar association mapping for quality traits was shown to be quite informative (Uitdewilligen et al. 2013).

In the current study, we have identified 59 marker trait associations for a number of traits measured under well-watered and water-limited conditions. Under water-limited conditions, our results were greatly influenced by maturity type, and many of the marker trait associations co-localized on chromosome 5 at the location of a major QTL that controls maturity in potato (Visker et al. 2003; Kloosterman et al. 2013). The co-localization of QTL for agro-morphological traits with yield and maturity in potato was also indicated by Anithakumari et al. (2012), Khan et al. (2015) and in Chapter 3 of this thesis under drought stress conditions. This QTL region was found to influence canopy development in potato grown in a field with two contrasting (low and high) nitrogen input level (Ospina 2016) and developmental traits under short day length with single trait linkage analysis (Hurtado et al. 2015). All these findings point to a pleiotropic effect of the gene underlying the QTLs on chromosome 5. Allelic variation of the underlying gene, Cyclic DOF Factor (CDF1), has strong influence on plant maturity and onset of tuberization (Kloosterman et al. 2013). This indicates maturity and tuberization in potato are closely related physiological traits and are controlled by CDF1 gene that mediates photoperiodic control of tuberization.

Our study used moderate drought stress conditions, while the previous studies applied severe drought stress, both under field and greenhouse conditions (Anithakumari et al. 2012, Khan et al. 2015; Chapter 3 of this thesis). In all these studies, the earliness locus on Chromosome 5 accumulated QTLs for agro-physiological traits. This implies that although the drought response in potato depends on severity and timing of water stress applied (Jefferies 1995), this locus remains a strong determinant for performance under drought even under 
different kinds of stress severity. The association of QTLs under different stress levels presents a great opportunity in improving potato for enhanced drought tolerance, although the strong linkage with maturity presents a challenge as well.

We have found QTLs for tuber yield, tuberization, plant height, shoot dry weight, and rootstolon dry weight co-localizing on chromosome 5 under water limited conditions, while only under well-watered conditions a QTL was identified on chromosome 5 for root-stolon dry weight. This agrees with Anithakumari et al. (2012), who detected several QTLs on chromosome 5 for drought tolerance and recovery traits, while only under well-watered conditions a QTL was reported for tuber yield (Table 5). Several of the QTLs detected on chromosome 5 under field condition for agro-morphological traits were detected both under drought stress and well-watered conditions (Chapter 3 of this thesis; Table 5). Moreover, Khan et al. (2015) reported many QTLs for agro-morphological and physiological traits on chromosome 5 under drought stress as well as well-watered conditions in a field experiment. In the latter study however, QTLs on chromosome 5 were detected only under water stress conditions in the drought stress experiment conducted in the greenhouse. This may suggest that the QTL region present on chromosome 5 is strongly influenced by the environmental (greenhouse or field) conditions in addition to the different environments created by the different levels of water availability. The expression of traits resulting from variation at the maturity locus are thus not only influenced by water availability but also by the other environmental factors (temperature, soil composition, day length, relative humidity, and light intensity, etc.) (Tuberosa 2012; Khan et al. 2015). Similarly, QTL (on chromosome 5) by environment interaction for day length was also reported for developmental traits (Hurtado Lopez 2012).

Under well-watered conditions, a QTL for tuber yield was detected on chromosome 10 and this marker is present in the same scaffold as a marker present in the QTL interval for $813 \mathrm{C}$ under drought stress conditions by Anithakumari et al. (2012). Carbon isotope composition $(\delta 13 C)$ is strongly correlated with water use efficiency in potato (Vos and Groenwold 1989), which is an important physiological trait under water-limited conditions likely to affect yield as well (Anitahkumari et al. 2012). Ospina (2016) reported a QTL for tuber number and tuber 
weight on chromosome 10 under low nitrogen supply, but this QTL did not seem to overlap with the QTL reported here.

Marker trait associations for plant height were detected on chromosome 4 and 5 under water limiting conditions. In the same scaffold on chromosome 4, QTLs for chlorophyll content and stem number were found under severe drought stress conditions and subsequent recovery, respectively by Anithakumari et al. (2012). These phenotypic QTLs also co-localized with eQTLs of transcription factor genes that have a role in abiotic stress response (Anithakumari 2011). Other phenotypic QTLs for root length, root dry weight, and $\delta 13 \mathrm{C}$ under stress conditions and tuber weight under recovery were reported on chromosome 4 (Anithakumari et al. 2012), and these were also located in close proximity of the QTL found in the present study. This suggests that this region on chromosome 4 supports both above- and belowground growth under stress conditions, and that it may be an important target for drought tolerance that is independent of maturity. The QTL on chromosome 4 reported for plant height under short day conditions in the field by Hurtado et al. (2015) did not overlap with the QTLs reported here. Marker trait associations were also detected on chromosome 4 for plant height and leaf area under well-watered conditions. Similarly, Ospina (2016) has reported a marker- trait association even in the same scaffold for canopy development traits. Other marker trait association for plant height under wellwatered conditions was detected on chromosome 8, 11 and 12. Chromosome 8 was reported to have a hotspot for QTLs controlling agro-morphological traits under wellwatered and drought stress conditions in both field and greenhouse in a different potato mapping population (Khan et al. 2015). Although we were not able to confirm there is an overlap in QTL positions, it may suggest that chromosome 8 carries a genomic region that controls growth traits under control and stress conditions.

QTLs for shoot dry weight and root-stolon dry weight were detected on chromosome 12 under drought stress conditions, in line with the strong positive correlation between these traits under these conditions. The co-localization of shoot dry weight and root-stolon dry weight on chromosome 5 and 12 suggests that this region may be used for selection of the difficult to phenotype underground traits of root and stolon growth. However, it should be noted that our experiments were done in pots, with root environment-restricted growing 
conditions, which may affect root growth as well as the effect of root biomass on shoot biomass and tuber yield. Tourneux et al. (2003) reported that genotypes with higher root dry mass had lower tuber yield in a pot experiment done in greenhouse. In contrast, under field conditions higher shoot biomass and root dry mass is reported to have a positive correlation with tuber yield under drought tress conditions (Lahlou and Ledent 2005; Chapter 3 of this thesis), indicating that plants in pots may not be able to benefit from improved root growth with higher shoot biomass and tuber yield. Breeding efforts to enhance drought tolerance in potato should therefore carefully consider the target growing conditions. Pot experiments allow for well-controlled drought conditions, but care should be taken in the interpretation of the results. Root dry mass under drought stress conditions was suggested as an important drought tolerance trait (Anithakumari et al. 2012), and root dry mass was indicated as an indirect selection criteria for enhanced drought tolerance in potato (Lahlou and Ledent 2005; Iwama 2008). However in the current study, root and stolon was collected as single trait for a practical reason. The QTLs identified on chromosome 12 are present on the same scaffold with the QTL identified for shoot fresh weight reported under control conditions (Anithakumari et al. 2011). Khan et al. (2015) has reported QTLs on chromosome 12 for dry biomass and dry stem leaf weight under drought stress conditions in a greenhouse experiment but we were not able to compare the QTL positions since different markers and populations were used. QTLs on chromosome 12 were also reported for plant height in short photoperiod environment from the single trait linkage anlaysis (Hurtado et al. 2015; Chapter 2 ), and for tuber number and canopy development under low nitrogen growing condition and for maximum tuber weight under high nitrogen input (Ospina 2016), but at different locations on this chromosome.

We have also identified a QTL on chromosome 9 for shoot dry weight under water stress conditions. On close proximity to this QTL, a QTL for shoot fresh weight was reported on chromosome 9 under a severe drought stress conditions of a greenhouse experiment (Anithakumari et al. 2012). Under field conditions, production of larger above ground biomass is suggested as a good drought tolerance trait for water stress conditions (Schittenhelm et al. 2006). However, in the current study shoot dry weight was negatively correlated with tuber yield, again indicating that in the current experimental set up with plants growing in pots, larger aboveground mass did not contribute to tuber yield. Under 
well-watered treatment QTLs for root-stolon dry weight were found on chromosome 3, 5 (at the maturity locus), and 6. QTLs on chromosome 3 and 6 for tuber number and weight were reported for tuber number and weight in two different levels (low and high) of nitrogen supply (Ospina 2016), but we did not observe an overlap in marker position. 


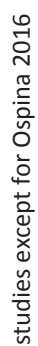

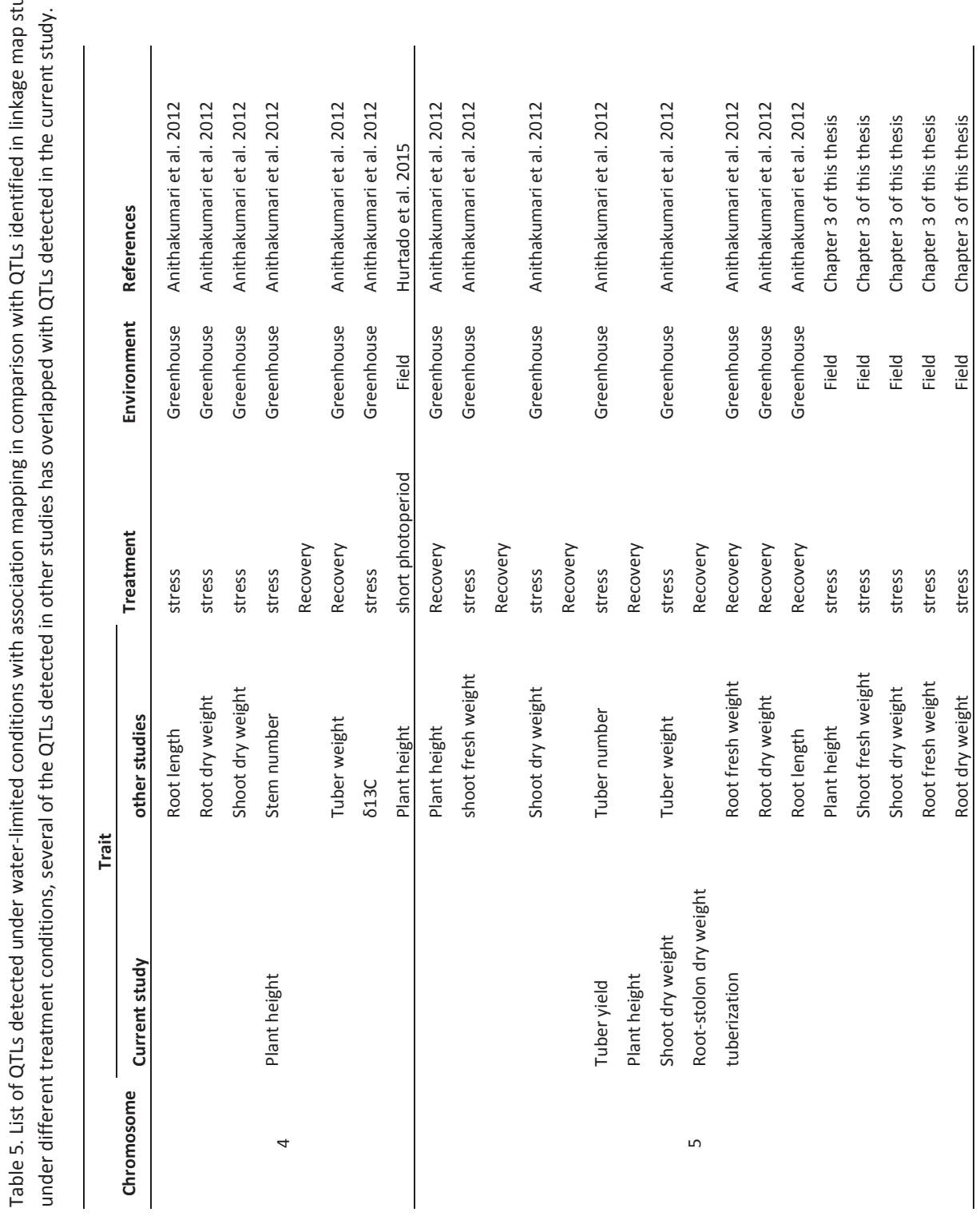




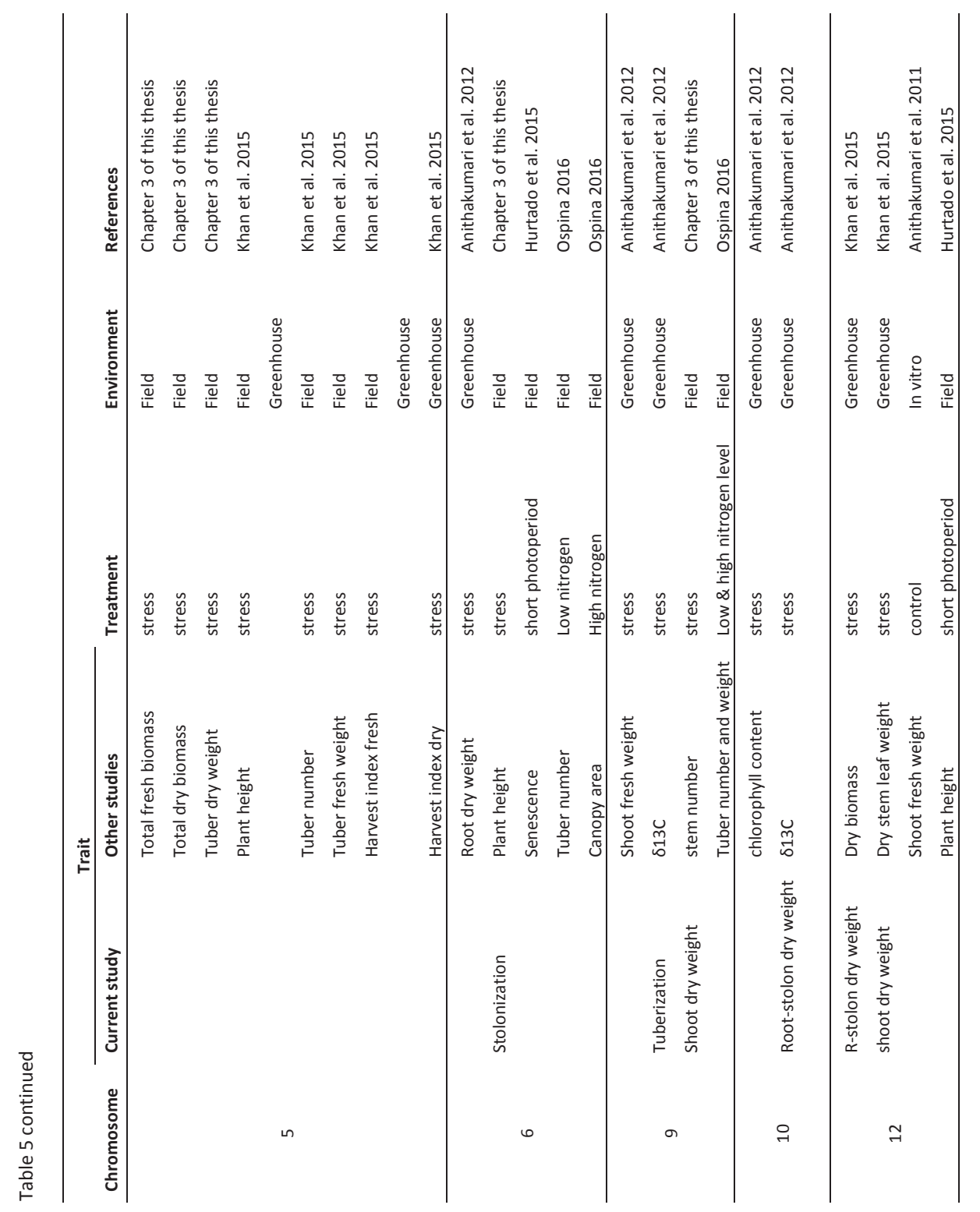


The effect of water limitation stress on tuber formation has been studied by many authors (Haverkort et al. 1990; Ewing and Struik 1992; Deblonde and Ledent 2001) and water limitation occurring at an early stage of development seemed to have a more pronounced effect on tuberization, resulting in more significant yield loss. In agreement with these findings, water stress delayed tuber formation in our study compared to well-watered conditions. Tuberization had a negative correlation with tuber yield under control as well as drought stress conditions indicating that in our experimental conditons, genotypes that took longer time to tuberize had less tuber yield. QTLs for time to tuberization were detected on chromosome 5 and 9, explained 19 and $25 \%$ of phenotypic variation observed under stress conditions. The QTL on chromosome 5 is likely to be functionally linked to the earliness locus as identified by Kloosterman et al. (2013). The CDF1 gene is photoperiodically controlled, and is an important mediator of the photoperiod signal to tuberization. By inhibition of CONSTANS, SP5G is stimulated, which in turn positively affects the tuberization signal SP6A. The SP6A signal is also sensitive to temperature, and tuberization is inhibited at high temperatures (Ewing 1981). Possibly, drought affects tuberization by interacting with components in this pathway, but the mechanism remains to be elucidated. The QTL detected on chromosome 9 for tuberization indicates that more factors in addition to CDF may influence the tuberization pathway under drought, and this QTL may be a starting point to elucidate the molecular mechanisms of the regulation of tuberization under drought stress conditions. The pleiotropic effect of the gene underlying this locus is exemplified by other QTL found at this location for chlorophyll content measured at 36 DAS under water stress conditions (Table 4). In addition, a QTL for shoot fresh weight and $\delta 13 \mathrm{C}$ was reported on chromosome 9 under severe drought stress conditions, while a QTL for plant height was identified under recovery conditions on the same chromosome (Anithakumari et al. 2012; Table 5). Carbon isotope discrimination $(\delta 13 \mathrm{C})$ represents the ratio between carbon assimilation and transpiration. Higher photosynthetic efficiency and delayed senescence that result into lower rate of chlorophyll reduction was shown to be associated with higher tuber yield under water-limited conditions (Ramirez et al. 2015). Similarly, Ospina (2016) has also identified QTL (in the same scaffold) on chromosome 9 for the potato canopy curve parameter representing the area under the declining phase of canopy development for low and high nitrogen input conditions, in a potato cultivar set that largely overlaps with the present study. The QTL region on chromosome 9 thus influences multiple traits 
(tuberization, chlorophyll content, $\delta 13 \mathrm{C}$, and canopy area) and warrants further investigation to confirm and understand the underlying gene function.

Our effort of applying association mapping to find genetic components contributing to drought tolerance in potato has resulted in the discovery of Interesting QTLS found in different chromosomes. These QTLs have explained moderate amount of the observed variation under mild stress conditions, and their validity was supported by QTLs found in previous studies. Although the drought response in potato is influenced by the level of stress applied (Jefferies 1995) the QTLs reported in this study largely overlaps with drought responses under different levels of stress, suggesting an inter-link in drought responses observed under moderate and severe drought stresses. These findings can support the design of efficient breeding strategies for enhanced drought tolerance in potato.

\section{References}

Anithakumari AM (2011) Genetic dissection of drought tolerance in potato. PhD Thesis, Wageningen University, Wageningen,

Anithakumari AM, Nataraja KN, Visser RG, van der Linden CG (2012) Genetic dissection of drought tolerance and recovery potential by quantitative trait locus mapping of a diploid potato population. Molecular breeding : new strategies in plant improvement 30 (3):1413-1429. doi:10.1007/s11032-012-9728-5

Blum A (1988) Plant breeding for stress environments. CRC Press, Inc.,

Boyer JS (1996) Advances in drought tolerance in plants. Advances in agronomy (USA)

Chapman SC, Chakraborty S, Dreccer MF, Howden SM (2012) Plant adaptation to climate change-opportunities and priorities in breeding. Crop Pasture Sci 63 (3):251-268. doi:10.1071/cp11303

D'hoop BB, Paulo MJ, Mank RA, van Eck HJ, van Eeuwijk FA (2008) Association mapping of quality traits in potato (Solanum tuberosum L.). Euphytica 161 (1-2):47-60. doi:DOI 10.1007/s10681-007-9565-5

Dai A (2011) Drought under global warming: a review. Wiley Interdisciplinary Reviews: Climate Change 2 (1):45-65

Deblonde P, Ledent J-F (2001) Effects of moderate drought conditions on green leaf number, stem height, leaf length and tuber yield of potato cultivars. European Journal of Agronomy 14 (1):31-41

Ersoz ES, Yu J, Buckler ES (2007) Applications of linkage disequilibrium and association mapping in crop plants. In: Genomics-assisted crop improvement. Springer, pp 97-119

Ewing E, Struik $P$ (1992) Tuber formation in potato: induction, initiation, and growth. Horticultural Reviews 14 (89):197

Ewing EE (1981) Heat stress and the tuberization stimulus. American Potato Journal 58 (1):31-49. doi:10.1007/bf02855378 
Gregory P, Simmonds L (1992) Water relations and growth of potatoes. In: The potato crop. Springer, pp 214-246

Hamilton JP, Hansey CN, Whitty BR, Stoffel K, Massa AN, Van Deynze A, De Jong WS, Douches DS, Buell CR (2011) Single nucleotide polymorphism discovery in elite North American potato germplasm. Bmc Genomics 12 (1):1

Haverkort A, Van de Waart M, Bodlaender Kd (1990) The effect of early drought stress on numbers of tubers and stolons of potato in controlled and field conditions. Potato Research 33 (1):89-96

Hijmans RJ (2003) The effect of climate change on global potato production. American Journal of Potato Research 80 (4):271-279

Hillel D, Rosenzweig C (2002) Desertification in relation to climate variability and change. Advances in agronomy 77:1-38

Hurtado Lopez PX (2012) Investigating genotype by environment and QTL by environment interactions for developmental traits in potato. PhD Thesis, Wageningen University, Wageningen.,

lerna A, Mauromicale G (2006) Physiological and growth response to moderate water deficit of off-season potatoes in a Mediterranean environment. Agricultural Water Management 82 (1-2):193-209. doi:10.1016/j.agwat.2005.05.005

Ingvordsen CH, Backes G, Lyngkjær MF, Peltonen-Sainio P, Jahoor A, Mikkelsen TN, Jørgensen RB (2015) Genome-wide association study of production and stability traits in barley cultivated under future climate scenarios. Mol Breeding 35 (3):1-14

Iwama K (2008) Physiology of the Potato: New Insights into Root System and Repercussions for Crop Management. Potato Research 51 (3-4):333-353. doi:10.1007/s11540-0089120-3

Jefferies R (1995) Physiology of crop response to drought. In: Potato ecology and modelling of crops under conditions limiting growth. Springer, pp 61-74

Kloosterman B, Abelenda JA, Gomez MdMC, Oortwijn M, de Boer JM, Kowitwanich K, Horvath BM, van Eck HJ, Smaczniak C, Prat S, Visser RGF, Bachem CWB (2013) Naturally occurring allele diversity allows potato cultivation in northern latitudes. Nature 495 (7440):246-250

Lahlou O, Ledent J-F (2005) Root mass and depth, stolons and roots formed on stolons in four cultivars of potato under water stress. European Journal of Agronomy 22 (2):159173. doi:10.1016/j.eja.2004.02.004

Lahlou O, Ouattar S, Ledent J-Fo (2003) The effect of drought and cultivar on growth parameters, yield

and yield components of potato. Agronomie 23 (3):257-268. doi:10.1051/agro:2002089

Long NV, Dolstra O, Malosetti M, Kilian B, Graner A, Visser RG, van der Linden CG (2013) Association mapping of salt tolerance in barley (Hordeum vulgare L.). Theoretical and applied genetics 126 (9):2335-2351

Malosetti M, van der Linden CG, Vosman B, van Eeuwijk FA (2007) A mixed-model approach to association mapping using pedigree information with an illustration of resistance to Phytophthora infestans in potato. Genetics 175 (2):879-889. doi:DOI 10.1534/genetics.105.054932

Monneveux P, Ramirez DA, Pino MT (2013) Drought tolerance in potato (S. tuberosum L.) Can we learn from drought tolerance research in cereals? Plant Science 205:76-86. doi:10.1016/j.plantsci.2013.01.011 
Ospina CA (2016) Nitrogen use efficiency in potato: an integrated agronomic, physiological and genetic approach. PhD Thesis, Wageningen University, Wageningen,

Pennisi E (2008) Plant genetics: The blue revolution, drop by drop, gene by gene. Science 320 (5873):171-173. doi:10.1126/science.320.5873.171

Rafalski JA (2010) Association genetics in crop improvement. Current opinion in plant biology 13 (2):174-180. doi:DOI 10.1016/j.pbi.2009.12.004

Rockström J, Barron J, Fox P (2003) Water productivity in rain-fed agriculture: challenges and opportunities for smallholder farmers in drought-prone tropical agroecosystems. Water productivity in agriculture: Limits and opportunities for improvement 85199 (669):8

Schafleitner R, Gutierrez R, Espino R, Gaudin A, Pérez J, Martínez M, Domínguez A, Tincopa L, Alvarado C, Numberto G, Bonierbale M (2007) Field Screening for Variation of Drought Tolerance in Solanum tuberosum L. by Agronomical, Physiological and Genetic Analysis. Potato Research 50 (1):71-85. doi:10.1007/s11540-007-9030-9

Schneider CA, Rasband WS, Eliceiri KW (2012) NIH Image to ImageJ: 25 years of image analysis. Nature methods 9 (7):671-675

Thiele G, Theisen K, Bonierbale M, Walker T (2010) Targeting the poor and hungry with potato science. Potato J 37 (3-4):75-86

Tuberosa R (2012) Phenotyping for drought tolerance of crops in the genomics era. Frontiers in physiology 3:347. doi:10.3389/fphys.2012.00347

Tuberosa R, Salvi S (2006) Genomics-based approaches to improve drought tolerance of crops. Trends in plant science 11 (8):405-412. doi:10.1016/j.tplants.2006.06.003

Uitdewilligen J, Wolters AMA, D'Hoop BB, Borm TJA, Visser RGF, van Eck HJ (2013) A NextGeneration Sequencing Method for Genotyping-by-Sequencing of Highly Heterozygous Autotetraploid Potato. Plos One 8 (5). doi:10.1371/journal.pone.0062355

Visker MH, Keizer LC, Van Eck HJ, Jacobsen E, Colon LT, Struik PC (2003) Can the QTL for late blight resistance on potato chromosome 5 be attributed to foliage maturity type? TAG Theoretical and applied genetics Theoretische und angewandte Genetik 106 (2):317325. doi:10.1007/s00122-002-1021-2

Voorrips RE, Gort G, Vosman B (2011) Genotype calling in tetraploid species from bi-allelic marker data using mixture models. Bmc Bioinformatics 12 (1):1

Vos J, Groenwold J (1989) Genetic differences in water-use efficiency, stomatal conductance and carbon isotope fractionation in potato. Potato Research 32 (2):113-121

Vos PG, Uitdewilligen JG, Voorrips RE, Visser RG, van Eck HJ (2015) Development and analysis of a 20K SNP array for potato (Solanum tuberosum): an insight into the breeding history. Theoretical and Applied Genetics 128 (12):2387-2401

Xue Y, Warburton ML, Sawkins M, Zhang X, Setter T, Xu Y, Grudloyma P, Gethi J, Ribaut J-M, Li W (2013) Genome-wide association analysis for nine agronomic traits in maize under well-watered and water-stressed conditions. Theoretical and applied genetics 126 (10):2587-2596 


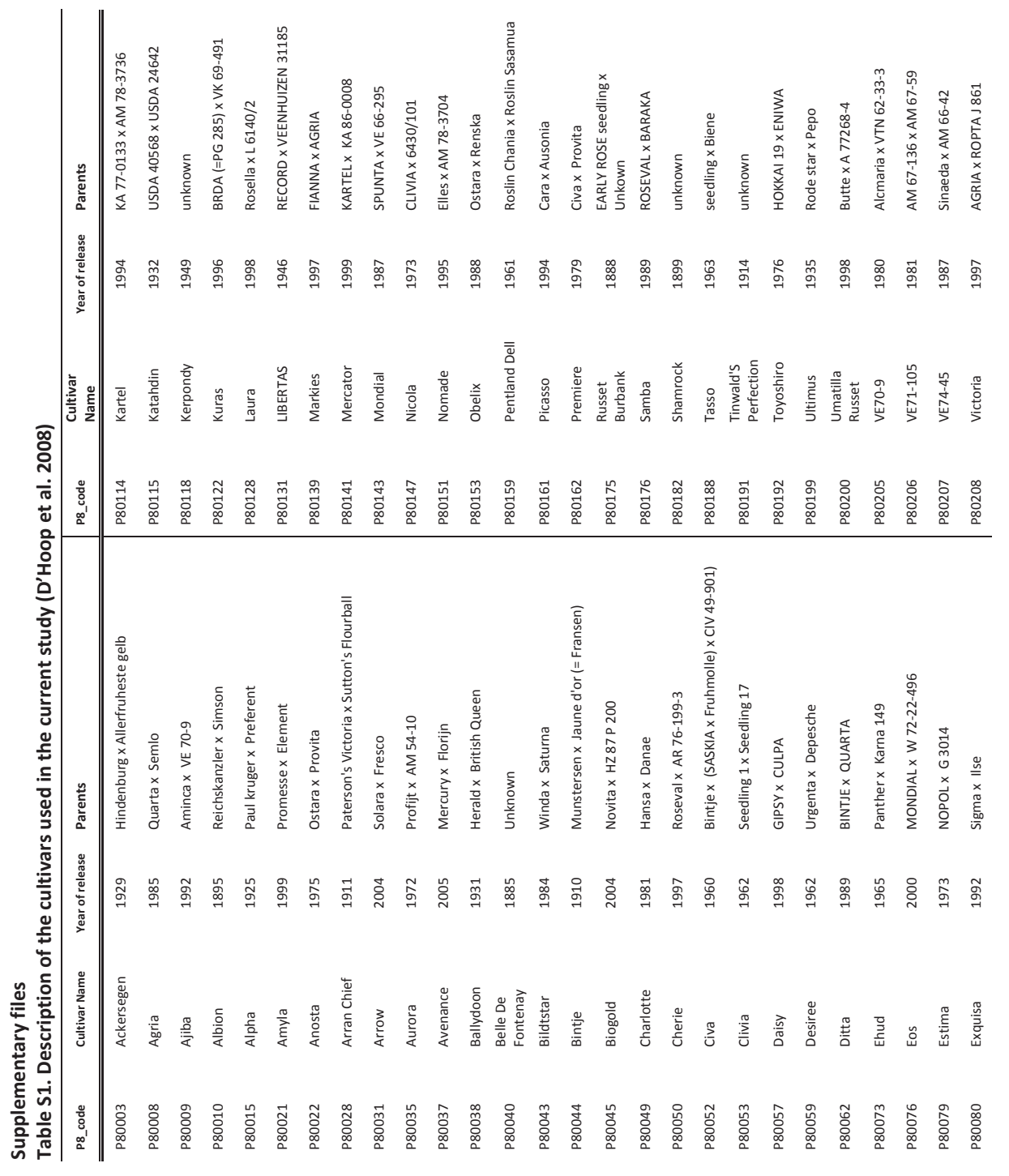




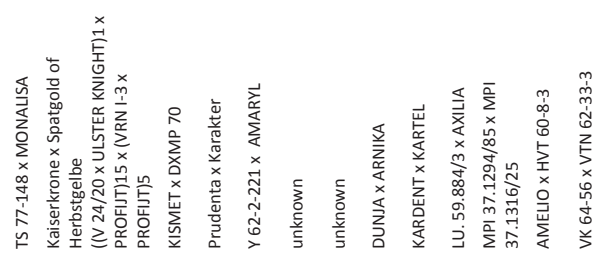

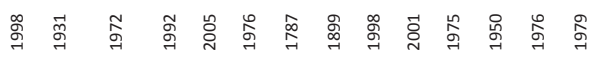

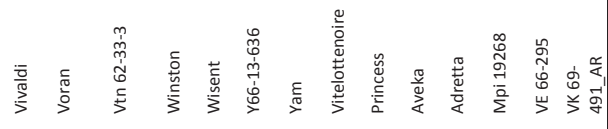

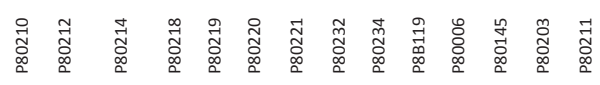

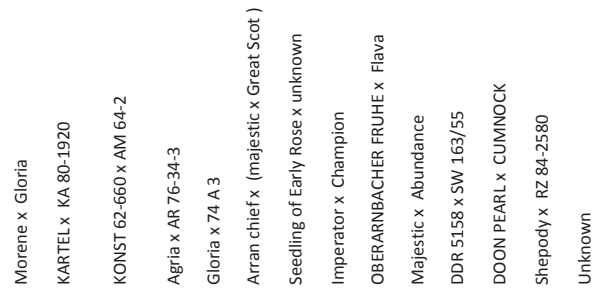

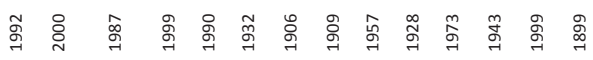

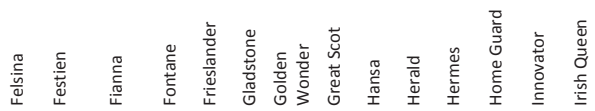

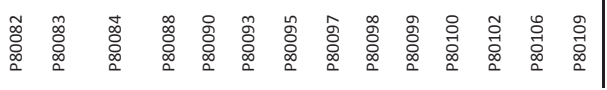


Table S2. Significant marker trait associations detected under well-watered conditions with $\log _{10}(P)$ value, Scaffold and the explained variation.

\begin{tabular}{|c|c|c|c|c|c|c|}
\hline Trait & chromosome & $\begin{array}{l}\text { Genome } \\
\text { position }\end{array}$ & Marker & scaffold & $-\log _{10}(P)$ & $\begin{array}{l}\text { Variance } \\
\text { explained }\end{array}$ \\
\hline Tuber yield & 10 & 707277375 & PotVar0122848 & PGGSC0003DMB000000506 & 4.16 & 23 \\
\hline \multirow[t]{5}{*}{ Plant height } & 4 & 283407138 & PotVar0116182 & PGSC0003DMB000000419 & 4.12 & 16 \\
\hline & 8 & 510726488 & solcap_snp_c1_9785 & PGSC0003DMB000000402 & 4.13 & 23 \\
\hline & 11 & 726858998 & PotVar0060023 & PGSC0003DMB000000133 & 4.31 & 22 \\
\hline & 11 & 726858998 & solcap_snp_c2_53678 & PGSC0003DMB000000133 & 4.31 & 22 \\
\hline & 12 & 776351708 & PotVar0069306 & PGSC0003DMB000000155 & 4.05 & 10 \\
\hline \multirow[t]{5}{*}{ Root-stolon dry weight } & 3 & 159370115 & PotVar0019246 & PGSC0003DMB000000039 & 4.01 & 17 \\
\hline & 5 & 315963223 & PotVar0078045 & PGSC0003DMB000000192 & 4.11 & 27 \\
\hline & 5 & 316045624 & PotVar0079081 & PGSC0003DMB000000192 & 4.19 & 26 \\
\hline & 5 & 316052012 & PotVar0079376 & PGSC0003DMB000000192 & 5.06 & 24 \\
\hline & 5 & 316611906 & solcap_snp_c2_50302 & PGSC0003DMB000000609 & 5.23 & 30 \\
\hline \multirow[t]{2}{*}{ Root-stolon dry weight } & 5 & 323792587 & PotVar0014413 & PGSC0003DMB000000027 & 4.42 & 21 \\
\hline & 6 & 419961527 & PotVar0070124 & PGSC0003DMB000000158 & 4.51 & 22 \\
\hline \multirow[t]{2}{*}{ Leafarea } & 4 & 291081071 & PotVar0099073 & PGSC0003DMB000000285 & 4.59 & 20 \\
\hline & 4 & 294711084 & PotVar0000462 & PGSC0003DMB000000002 & 4.10 & 13 \\
\hline CC16DASY & 1 & 46273159 & PotVar0132293 & PGSC0003DMB000000674 & 4.48 & 13 \\
\hline \multirow[t]{4}{*}{ CC36DASY } & 10 & 652427095 & PotVar0108085 & PGSC0003DMB000000338 & 4.27 & 21 \\
\hline & 11 & 717880690 & PotVar0064142 & PGSC0003DMB000000148 & 4.44 & 24 \\
\hline & 12 & 827167202 & solcap_snp_c2_39393 & PGSC0003DMB000000477 & 4.43 & 20 \\
\hline & 12 & 832117394 & PotVar0053054 & PGSC0003DMB000000114 & 4.09 & 14 \\
\hline CC36DASM & 1 & 47097440 & PotVar0071528 & PGSC0003DMB000000169 & 4.10 & 6 \\
\hline CC49DASM & 2 & 139930088 & solcap_snp_c2_7559 & PGSC0003DMB000000244 & 4.79 & 21 \\
\hline
\end{tabular}


Table S3. Significant marker trait associations detected under water-limited conditions with $\log _{10}(P)$ value, Scaffold and the explained variation.

\begin{tabular}{|c|c|c|c|c|c|c|}
\hline Trait & Chromosome & $\begin{array}{l}\text { Genome } \\
\text { Position }\end{array}$ & Marker & Scaffold & $-\log _{10}(P)$ & $\begin{array}{l}\text { Variance } \\
\text { explained }\end{array}$ \\
\hline \multirow[t]{2}{*}{ Tuber Yield } & 5 & 316278656 & PotVar0080213 & PGSC0003DMB000000192 & 4.40 & 16 \\
\hline & 5 & 363238192 & PotVar0034717 & PGSC0003DMB000000192 & 4.03 & 6 \\
\hline \multirow[t]{3}{*}{ Plant height } & 4 & 284848878 & solcap_snp_c2_48808 & PGSC0003DMB000000234 & 4.13 & 11 \\
\hline & 4 & 287670623 & PotVar0084419 & PGSC0003DMB000000213 & 4.44 & 17 \\
\hline & 5 & 315369759 & PotVar0025609 & PGSC0003DMB000000051 & 4.33 & 21 \\
\hline \multirow[t]{7}{*}{ Shoot dry weight } & 5 & 315369759 & PotVar0025609 & PGSC0003DMB000000051 & 4.38 & 25 \\
\hline & 9 & 620329173 & solcap_snp_c1_6476 & PGSC0003DMB000000229 & 4.31 & 18 \\
\hline & 12 & 830509804 & PotVar0018338 & PGSC0003DMB000000034 & 5.23 & 15 \\
\hline & 12 & 830859701 & PotVar0018262 & PGSC0003DMB000000034 & 5.06 & 15 \\
\hline & 12 & 831892141 & PotVar0053356 & PGSC0003DMB000000114 & 5.25 & 17 \\
\hline & 12 & 832115480 & PotVar0053166 & PGSC0003DMB000000114 & 5.95 & 17 \\
\hline & 12 & 832700777 & PotVar0052560 & PGSC0003DMB000000114 & 4.07 & 9 \\
\hline \multirow[t]{6}{*}{ Root-stolon dry weight } & 5 & 315963223 & PotVar0078045 & PGSC0003DMB000000192 & 4.95 & 36 \\
\hline & 5 & 315976753 & PotVar0078561 & PGSC0003DMB000000192 & 4.54 & 33 \\
\hline & 5 & 316045624 & PotVar0079081 & PGSC0003DMB000000192 & 4.44 & 32 \\
\hline & 5 & 316052012 & PotVar0079376 & PGSC0003DMB000000192 & 5.35 & 31 \\
\hline & 10 & 697993735 & solcap_snp_c1_15594 & PGSC0003DMB000000106 & 4.67 & 19 \\
\hline & 12 & 830509804 & PotVar0018338 & PGSC0003DMB000000034 & 4.20 & 13 \\
\hline \multirow[t]{2}{*}{ Stolonization } & 3 & 202526012 & solcap_snp_c2_55283 & PGSC0003DMB000000126 & 4.35 & 5 \\
\hline & 6 & 403936989 & solcap_snp_c2_11314 & PGSC0003DMB000000156 & 4.27 & 20 \\
\hline \multirow[t]{3}{*}{ Tuberization } & 5 & 316278656 & PotVar0080213 & PGSC0003DMB000000192 & 4.92 & 19 \\
\hline & 9 & 623920945 & PotVar0051195 & PGSC0003DMB000000110 & 4.38 & 20 \\
\hline & 9 & 623998221 & solcap_snp_c1_12802 & PGSC0003DMB000000110 & 4.98 & 25 \\
\hline CC36DASY & 12 & 833614485 & solcap_snp_c2_5474 & PGSC0003DMB000000566 & 4.25 & 15 \\
\hline \multirow[t]{3}{*}{ CC36DASM } & 8 & 513405102 & PotVar0108992 & PGSC0003DMB000000341 & 4.06 & 10 \\
\hline & 9 & 623998221 & solcap_snp_c1_12802 & PGSC0003DMB000000110 & 4.35 & 20 \\
\hline & 9 & 624088981 & solcap_snp_c1_6192 & PGSC0003DMB000000110 & 4.60 & 19 \\
\hline \multirow[t]{8}{*}{ CC49DASY } & 6 & 427408071 & PotVar0040034 & PGSC0003DMB000000087 & 4.00 & 12 \\
\hline & 9 & 630453530 & PotVar0107676 & PGSC0003DMB000000334 & 4.17 & 11 \\
\hline & 9 & 630453545 & solcap_snp_c2_22003 & PGSC0003DMB000000334 & 4.17 & 11 \\
\hline & 9 & 630453623 & PotVar0107672 & PGSC0003DMB000000334 & 5.89 & 10 \\
\hline & 9 & 630884244 & solcap_snp_c2_22069 & PGSC0003DMB000000439 & 4.67 & 10 \\
\hline & 9 & 631301143 & solcap_snp_c2_46777 & PGSC0003DMB000000384 & 4.12 & 12 \\
\hline & 9 & 631374892 & solcap_snp_c2_46796 & PGSC0003DMB000000384 & 4.91 & 10 \\
\hline & 9 & 631477952 & solcap_snp_c2_29310 & PGSC0003DMB000000384 & 4.37 & 9 \\
\hline \multirow[t]{2}{*}{ CC49DASM } & 6 & 564384310 & solcap_snp_c2_16997 & PGSC0003DMB000000048 & 4.95 & 16 \\
\hline & 8 & 421984583 & PotVar0090783 & PGSC0003DMB000000246 & 4.23 & 15 \\
\hline
\end{tabular}





\section{Chapter 5}

\section{Relationship between soil ground cover and tuber yield production in potato under drought stress conditions}

Biructawit B. Tessema ${ }^{1,2}$, Richard GF Visser ${ }^{1}$, C. Gerard van der Linden ${ }^{1}$

\footnotetext{
${ }^{1}$ Wageningen UR Plant Breeding, Wageningen University \& Research, PO Box 386, $6700 \mathrm{AJ}$ Wageningen, the Netherlands

${ }^{2}$ The graduate school of Experimental Plant Sciences (EPS), Wageningen University, Wageningen, the Netherlands
} 


\begin{abstract}
Potato is recognized as an efficient water user yet shortage of water during its early developmental stage has a significant effect on tuber yield. Water stress affects leaf growth and expansion, which will result in lower rate of canopy development and smaller canopies. In order to assess the effect of drought stress on canopy development of potato, we carried out field drought experiments using selections from a diploid potato backcross mapping population (CXE). Drought stress was applied at the stage of stolon formation. Canopy development was measured as the percentage of soil cover by green leaves. Canopy data was analysed using a model that described the canopy development curve expressed in thermal time, based on the beta function and estimated cardinal temperature. Under stress conditions, tuber yield and tuber number were greatly reduced. Drought stress also significantly affected curve-fit parameters. Water shortage reduced maximum soil cover $(V x)$, which was correlated with a decrease in tuber yield. Drought also induced senescence by shortening the time at which canopy starts to decline (t2). Area under the canopy development curve $(A \cup C)$ was generally lower under water-limited conditions and it was influenced by maturity type. The correlation observed between canopy curve parameters and tuber yield was less strong under drought stress conditions compared to well-watered conditions, indicating that under drought, other factors in addition to canopy development control tuber yield.
\end{abstract}

Keywords: canopy development, drought stress, potato 


\section{Introduction}

The potato crop stands out for its productive water use, yielding more food per unit of water than any other major crop. Yet, this crop is sensitive to even short periods of water shortage affecting its canopy formation and tuber yield (Lahlou et al. 2003). Drought stress affects potato canopy development by decreasing leaf size and leaf expansion rate while limiting formation of new leaves and increasing the rate of senescence (Jefferies and MacKerron 1993; Fleisher et al. 2008). The reduction in canopy growth will have an influence on the amount of radiation intercepted and subsequently its conversion to dry matter production (Jefferies 1995a) and thus results in delayed tuber initiation and reduced tuber yield production (Lahlou et al. 2003).

The development of potato canopy is divided into three main phases (Haverkort 2007; Khan 2012). The first phase represents emergence to tuber initiation, the second phase covers the period from tuber initiation and tuber bulking until start of senescence and the third phase represents senescence and end of crop growth. Under non-stressed conditions, the growth rate of potato depends on the amount of radiation intercepted by the foliage and there is a linear relationship between canopy cover and tuber yield (Allen and Scott 1980; Van Oijen 1991). The proportion of intercepted radiation by the canopy cover can be estimated by measuring the percentage of soil cover (Haverkort et al. 1991). The rate and duration of canopy growth together with the rate of canopy senescence determines canopy cover during the growth season of potato (Struik et al. 1990). Differences in growth, duration of maximum green canopy cover and its senescence rate affect the rate of dry matter accumulation through differences in light interception and utilization of intercepted radiation (Van Delden et al. 2001; Khan 2012). Under optimal conditions, the longer the growth period of canopy cover the higher the tuber yield through better interception of incoming radiation (Martin 1995). Longer duration of canopy development allows more assimilates to be produced and to be partitioned into tubers, which determines tuber yield. However, abiotic factors such as drought stress can reduce canopy cover, which will affect radiation interception to varying degrees (Haverkort 2007). Moreover, among other factors, dry matter partitioning into tubers can be influenced by drought stress (Haverkort 2007). 
Several studies have suggested a positive relationship between canopy development and tuber yield production under water-limited conditions (Jefferies and Mackerron 1993; Deblonde and Ledent 2001; Schittenhelm et al. 2006). These studies suggest that genotypes that can sustain their aboveground biomass under water-limited conditions are able to produce higher tuber yields. However, there is still lack of information on different aspects of canopy growth under drought stress conditions and its relationship to tuber yield.

The presence of large genotypic differences in potato for canopy cover allows improving potato for tuber yield since differences in yield can be attributed to variation in canopy cover (Jefferies and Mackerron 1993; Khan 2012; Ospina 2016). Variations in canopy cover over time were reported to be influenced by maturity class, explaining yield differences in potato cultivars grown under different levels of nitrogen application (Ospina 2016). Maturity type is classified as a major determining factor for total canopy cover and dry matter yield, where late maturing genotypes have higher canopy cover and tuber dry matter yield (Ospina et al. 2014; Ospina 2016). The differences in yield were indicated to come from differences in cumulative light absorption and light use efficiency.

Canopy cover estimation based on soil coverage was shown to be a powerful tool to study factors that may affect plant developmental traits in potato (Khan 2012; Ospina et al. 2014). A quantitative approach to model potato canopy cover dynamics as a function of thermal time and soil coverage was developed by Khan (2012) following a beta function (Yin et al. $2003,2009)$ which allowed to divide the canopy cover development pattern into distinct stages (canopy build-up phase, maximum cover phase, and canopy decline phase). The application of model-derived canopy curve parameters was shown to explain the response of canopy cover development to different levels of nitrogen application (Ospina et al. 2014; Ospina 2016). Genetic variation for canopy development curve parameters that had biological relevance in explaining canopy growth and tuber yield under contrasting (high and low) level of nitrogen applications was reported (Ospina 2016).

In the present study, we used this quantitative approach to model potato canopy cover dynamics following the procedure of (Khan 2012) in order to assess the relationship between canopy cover and tuber yield production under drought stress conditions using a 
selected subset of the CXE diploid potato mapping population. Our results indicate that although the relationship between soil ground cover with tuber yield is still present under water-limited conditions, it is less strong when compared to well-watered conditions, suggesting that additional factors affecting tuber yield play a role under drought.

\section{Material and Methods}

\section{Plant materials}

We used 20 genotypes that were selected from a drought stress experiment conducted using the CXE diploid potato populations in 2010 under field conditions (Chapter 3 of this thesis). The CxE potato mapping population is the result of a cross of two diploid potato clones, USW53373.3 coded C and 77.2102.37 coded E. Clone C is a hybrid between S. phureja PI 225696.1 and the S. tuberosum dihaploid USW42. Clone E is a cross between clone $\mathrm{C}$ and the $\mathrm{S}$. vernei $-\mathrm{S}$. tuberosum backcross clone VH34211. The full description of the population can be obtained from (Celis-Gamboa 2002). We have selected 10 early and 10 intermediate maturing genotypes. The 10 genotypes of each maturity class consisted of genotypes with contrasting responses under drought conditions, number of tubers and yield (Table S1), as determined in the trials described in Chapter 3.

\section{Experiment setup}

A drought stress experiment was conducted at Melkassa Agricultural Research Institute, Ethiopia. The semi-arid environment at Melkassa $\left(8024^{\prime} \mathrm{N} 39021^{\prime} \mathrm{E}\right.$ coordinates) has an average day temperature of $280 \mathrm{C}$, annual rain fall of $928 \mathrm{~mm}$ and is situated at 1550 meters above sea level (masl), with clay loam soil. A split plot design with 2 replications for each treatment was used. Potato seed tubers (8 plants per genotype per replicate) were planted with a spacing of $0.75 \mathrm{~m}$ between rows and $0.30 \mathrm{~m}$ between plants within a row. The recommended rate of UREA (165kg/ha) and DAP (Diammonium phosphate) $(195 \mathrm{~kg} / \mathrm{ha})$ was applied. The drought stress treatment was started at the stage ofstolon initiation (determined by visual inspection of stolon growth in the upper layer of the soil) at 38 days after planting (DAP). Drought stress was created by completely withholding water for two of the replications. The remaining 2 replications were well-watered throughout the experiment. 


\section{Phenotyping}

Plant emergence was recorded per plot from the moment first leaves were visible. Days to emergence (DAE) for each plot were taken as the day when $50 \%$ of the plants had emerged. After emergence, green canopy cover (\%) was measured in 3 to 5 day intervals to a total of 19 time points in each plot. The first four measurements of canopy cover were done before applying drought stress. For measuring canopy cover, we used a rectangle aluminum grid with a dimension of $0.75 \times 0.6 \mathrm{~m}$, which is the planting distance between rows and $2 x$ the between-plant distance. The grid was partitioned into 100 equal squares. Green canopy cover was measured by putting the grid above the canopy of two individual plants at a time and counting the squares that were more than half filled with green cover, expressed as a percentage of the total number of squares. Chlorophyll content was measured on young fully expanded leaflets on 56,70 , and 84 days after planting $(18,32,46$ days after stress). At harvest, 104 days after planting (66 days after stress), tuber weight and tuber number were recorded and the data was expressed per $\mathrm{m} 2$. This was differently expressed than Chapter 3 because canopy cover was measure as how much of the area $(0.7 \mathrm{~m} \times 0.6 \mathrm{~m})$ was covered by green leafs.

\section{Thermal days}

The beta thermal times for each canopy assessment day were calculated from the emergence date for each plot using the beta-function as described by Yin et al. (2003). The cardinal temperatures for potato as determined by (Khan et al. 2013) were used in calculating thermal days. Temperature was recorded every three hours. The non-linear relationship between temperature $(T$ in $\circ C$ ) and rate of growth $g(T)$ is described by equation (1). The three cardinal temperatures for phenological development of potato (base $\left(\mathrm{T}_{\mathrm{b}}=5.5\right)$, optimum $\left(\mathrm{T}_{\mathrm{o}}=23.4\right)$, and ceiling $\left.\left(\mathrm{T}_{\mathrm{c}}=34.6\right)\right)$ and temperature response curvature coefficient $\left(C_{t=1}\right.$ 1.6) was used as described by (Khan 2012; Khan et al. 2013).

$$
g(T)=\left[\left(\frac{T_{c}-T}{T_{C}-T_{o}}\right)\left(\frac{T-T_{b}}{T_{o}-T_{b}}\right)^{\left(\frac{T_{o}-T_{b}}{T_{C}-T_{o}}\right)}\right]^{c_{t}}
$$




\section{Curve fitting}

The calculated beta thermal time (BTT) with the canopy cover data from each replicate plot was used to curve fit with SAS following the non-linear NOLIN procedure. The curve fit with an estimated value explaining the different phases of canopy development was derived from equations (2) to (4)(Yin et al. 2003). For the different phases of canopy development (Figure 1 ), this procedure produced five parameter estimates describing the best fit curve; four $t$ parameters expressed in thermal days (td) and maximum canopy cover ( $\mathrm{Vx}$ ) expressed in percentage. The four t parameters are tm1 (inflexion point in the growing phase of the curve), t1 (time at which maximum canopy cover is reached), t2 (start of senescence), and te (time at which the canopy had died) while $\mathrm{Vx}$ is maximum canopy cover.

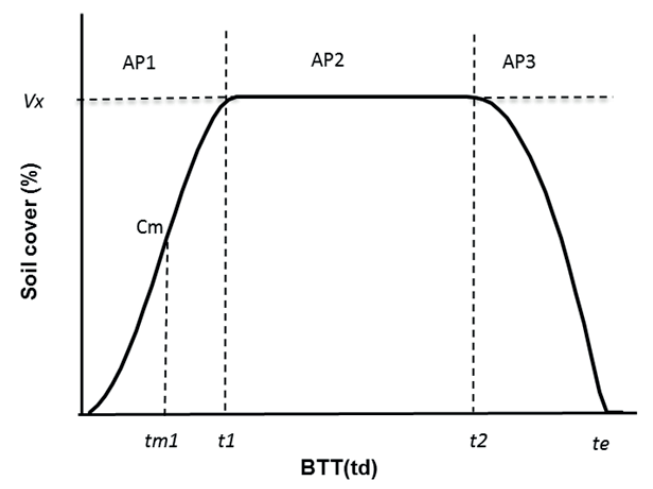

Figure 1. Canopy development curve showing the percentage of soil cover across the potato growing period in beta thermal time (BTT) expressed as thermal days (td). The canopy development parameters $(\mathrm{Cm}, \mathrm{tm} 1, \mathrm{t} 1, \mathrm{t} 2, \mathrm{te})$ and area under the three developmental phases (AP1, AP2, AP3) are described in the main text.

$$
\begin{aligned}
& V=V x\left(1+\frac{t_{1}-t}{t_{1}-t_{m 1}}\right)\left(\frac{t}{t_{1}}\right)^{\frac{t_{1}}{t_{1}-t_{m 1}}} \text { with } 0 \leq t \leq t_{1} \\
& V=V_{x} \text { with } t_{1} \leq t \leq t_{2}
\end{aligned}
$$




$$
V=V_{x}\left(\frac{t_{e}-t}{t_{e}-t_{2}}\right)\left(\frac{t+t_{1}-t_{2}}{t_{1}}\right)^{\frac{t_{1}}{t_{e}-t_{2}}} \text { with } t_{2} \leq t \leq t_{e}
$$

\section{Calculated variables}

From the canopy development model, parameters that express the area and duration of the different developmental stages were calculated (Khan 2012; Khan et al. 2013). The duration for the maximum canopy cover is t2-t1, and the time taken for the declining phase is te-t2. Using the curve parameter estimates that explain durations, the maximum progression rate was calculated using equation (5). The areas under the canopy development curve (Figure 1): phase 1 (AP1), phase 2 (AP2), and phase 3 (AP3) were calculated using the equations (6) to (8). Total area under the curve (AUC) was calculated by summing up the areas of the three developmental phases.

$$
\begin{aligned}
& C_{m}=\left(\frac{2 t_{1}-t_{m 1}}{t_{1}\left(t_{1}-t_{m 1}\right)}\right)\left(\frac{t_{m 1}}{t_{1}}\right)^{\frac{t_{m 1}}{t_{1}-t_{m 1}}} V_{x} \\
& A P 1=V_{x}\left\lceil\frac{2 t_{1}\left(t_{1}-t_{m 1}\right)}{3 t_{1-2}-2 t_{m 1}}\right\rceil \\
& A P 2=V_{x}\left(t_{2}-t_{1}\right) \\
& A P 3=\frac{V_{x}\left(t_{e}-t_{2}\right)}{2 t_{e}-2 t_{2}+t_{1}}\left\lceil\left(t_{e}-t_{2}+t_{1}\right)\left(\frac{\left(t_{e}-t_{2}+t_{1}\right)}{t_{1}}\right)^{\frac{t_{1}}{t_{e}-t_{2}}}-2 t_{1}\right]
\end{aligned}
$$




\section{Results}

\section{Treatment effect on canopy development}

Phenotyping for the 20 selected genotypes was done and resulted in different curve fit, depending not only on the environmental condition (drought stress or well-watered) but also on the maturity type. In Figure 2 some typical curves are shown.

The effect of drought stress was significant for most of the canopy development curve estimated parameters as well as for the agronomic traits (Table 1). The drought stress treatment started at thermal day 14.8. Under drought stress conditions, tm1 was shorter (thermal day 13.5 vs 17.3 under well-watered conditions), and t1 was also shorter under drought stress (21.5) than well-watered (28.2) conditions. The significant difference for t1 due to treatment suggests that the first phase of canopy development, building up to a maximum canopy, was affected in response to drought stress. The start of senescence was much faster under drought stress conditions ( $\mathrm{t} 2=27.3)$ compared to well-watered conditions (t2= 50). Time taken to complete the life cycle (te) was also reduced in response to water stress (Table 1; Figure 2). Both canopy development curve parameters t2 and te showed significant differences in the response to treatment ( $P=0.031$ and 0.017 , respectively) and in genotype by treatment interaction.

The duration t2-t1, which is the time that soil coverage stays at its maximum, showed a small reduction in response to the water shortage and was non-significant. We observed that even under control conditions the duration of maximum soil coverage was short. Other experiments done using the whole set of CXE genotypes under Ethiopian conditions, were reported to also have a short duration (t2-t1) for maximum soil cover under control conditions (personal communication). The maximum soil coverage $(\mathrm{Vx})$ was greatly reduced in response to water stress, demonstrating the negative effect of drought on the establishment of a full canopy in particular, which also strongly reduces total capacity of light interception of the potato plants. $\mathrm{Vx}$ also showed highly significant interaction between genotype and treatment 
The average values for AP1, AP2, AP3, and AUC were reduced in response to drought stress (Table 1). Area under the curve for the first (AP1) and third (AP3) developmental phase of canopy showed significant differences in response to drought, while the second developmental phase (AP2) was not significantly different between drought and control conditions. Total area under the curve (AUC) was affected by drought stress, showing significant effects for genotype, drought and their interaction. These results indicate that under the trial conditions, drought affects both the total duration of the canopy development and the maximum canopy cover $\mathrm{Vx}$. The strongest effect on light interception capacity was the reduction of $\mathrm{Vx}$ and of $\mathrm{t} 2-\mathrm{t} 1$.

The mean values for the agronomic traits tuber number and tuber yield were reduced in response to drought (Table 1). Under well-watered conditions, tuber number and yield had mean values of 56 tubers $/ \mathrm{m} 2$ and $881 \mathrm{~g} / \mathrm{m} 2$, respectively, while under water-limited conditions, the mean value for tuber number was 10 tubers $/ \mathrm{m} 2$ and $107 \mathrm{~g} / \mathrm{m} 2$ for tuber yield. Chlorophyll content measured 56 days after planting (DAP) showed a significant effect of drought. However, chlorophyll content measured at 70 and 84 DAP were not significantly affected by drought.

The performance of the genotypes under well-watered and drought stress conditions for canopy development curve parameters and agronomic traits along with their heritability is presented in Table 2. Under drought stress conditions, genotypes showed significant difference for all of the canopy development curve parameters except for AP2 and t2-t1 (Table 2), indicating that there is genetic variation between the CXE genotypes for drought tolerance. The significant difference among genotypes for drought tolerance was observed in a previous field drought stress experiments (Chapter 3 of this thesis). Under well-watered conditions, genotypes showed significant variation for most traits as well, except for AP3 and tuber weight. All the significant parameters (tm1, t1, Vx, te, Cm, AP1, AP3, AUC, te-t2) under stress conditions showed high heritability values, indicating that that there is a strong genetic component controlling the phenotypic variation of these parameters and the influence of environmental variation is relatively small. Heritability values were also high under well-watered conditions. Chlorophyll content, tuber number and weight showed high heritabilities under drought stress conditions. 
Table 1. Canopy development curve parameters and agronomic traits with their mean value under drought stress (DS) and well-watered (WW) conditions, p-values of treatment, genotype, and treatment by genotype interactions from analysis of variance.

\begin{tabular}{lrrccc}
\hline \multirow{2}{*}{ Traits } & \multicolumn{2}{c}{ Mean } & & & \\
\cline { 2 - 4 } & WW & DS & $\begin{array}{c}\text { Treatment } \\
(\mathrm{T})\end{array}$ & $\begin{array}{c}\text { Genotype } \\
(\mathrm{G})\end{array}$ & TxG \\
\hline$t m 1(t d)$ & 17.3 & 13.5 & 0.018 & $<0.001$ & 0.011 \\
$t 1(t d)$ & 28.2 & 21.5 & 0.009 & $<0.001$ & $<0.001$ \\
$t 2(t d)$ & 32.8 & 24.4 & 0.031 & $<0.001$ & 0.002 \\
$T e(t d)$ & 52.1 & 42 & 0.017 & $<0.001$ & 0.003 \\
$V x(\%)$ & 50 & 27 & 0.005 & $<0.001$ & $<0.001$ \\
$t 2-t 1(t d)$ & 4.6 & 2.9 & 0.075 & 0.006 & 0.221 \\
$t e-t 2(t d)$ & 19.3 & 17.6 & 0.383 & 0.001 & 0.016 \\
$C m(\% C C / t d)$ & 3 & 2.2 & 0.01 & $<0.001$ & $<0.001$ \\
$A P 1(\% C C . t d)$ & 633.6 & 254.5 & 0.007 & $<0.001$ & $<0.001$ \\
$A P 2(\% C C . t d)$ & 277.5 & 79.4 & 0.075 & 0.006 & 0.221 \\
$A P 3(\% C C . t d)$ & 647.1 & 320.8 & 0.013 & $<0.001$ & 0.037 \\
AUC $(\% C C . t d)$ & 1508.2 & 654.7 & 0.005 & $<0.001$ & $<0.001$ \\
TuNr $\left(\mathrm{Tb} / \mathrm{m}^{2}\right)$ & 55 & 10 & 0.002 & $<0.001$ & 0.003 \\
TuWt $\left(\mathrm{g} / \mathrm{m}^{2}\right)$ & 881.3 & 107.7 & 0.001 & 0.001 & 0.04 \\
CC56DAP & 40 & 47 & 0.012 & $<0.001$ & 0.377 \\
CC70DAP & 37 & 42 & 0.116 & $<0.001$ & 0.340 \\
CC84DAP & 36 & 40 & 0.115 & $<0.001$ & $<0.001$ \\
\hline
\end{tabular}

$t d=$ thermal days, \%CC= \%canopy cover, $\mathrm{Tb} \#=$ tuber number 
둔

$\stackrel{\text { ษ }}{-}$

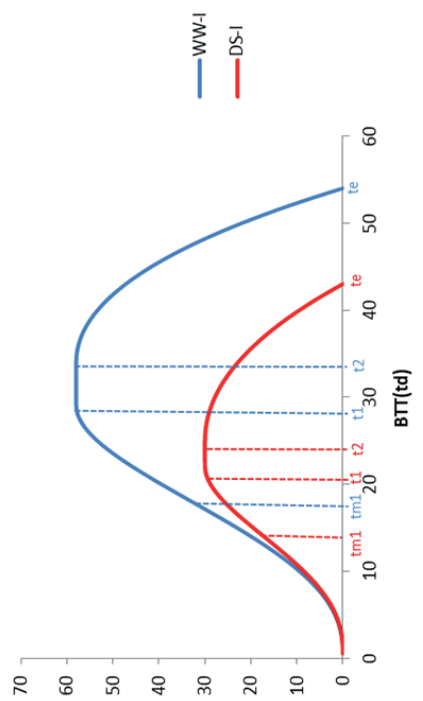

dै

츤

๑)

जิ

ज

若韋

응 듬

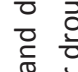

원

ע

离

Ii

î
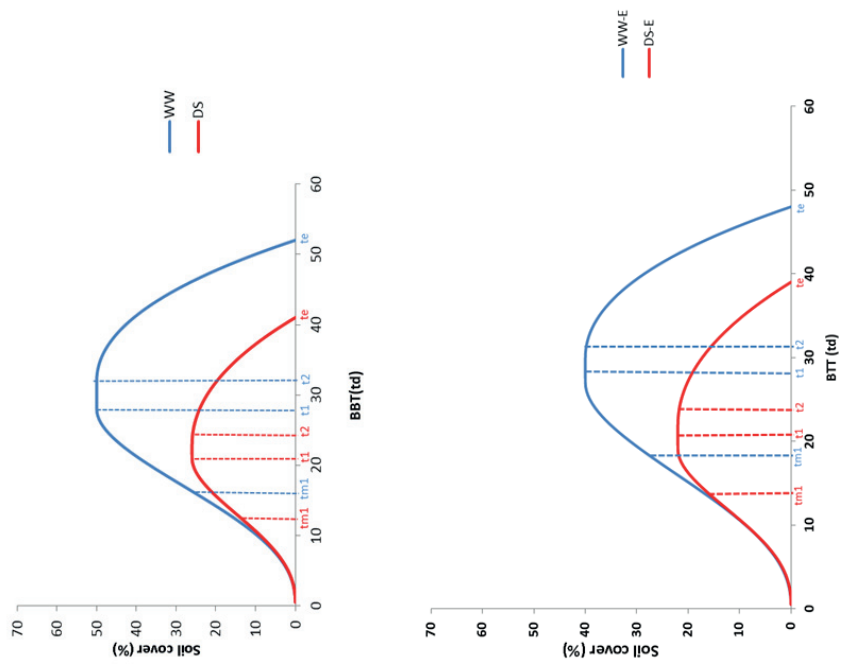

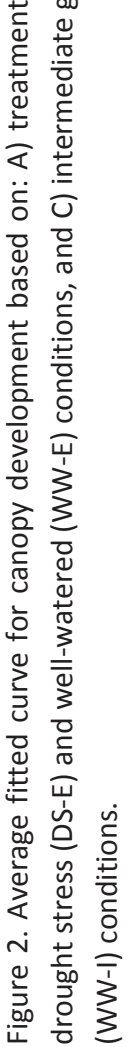


Table 2. P-values and heritabilities from analysis of variance for different canopy curve parameters, chlorophyll content and yield traits measured under drought stress and wellwatered conditions

\begin{tabular}{|c|c|c|c|c|}
\hline \multicolumn{3}{|c|}{ Well-watered } & \multicolumn{2}{|c|}{ Drought stress } \\
\hline traits & P-value & $\mathrm{H}^{2}$ & P-value & $\mathrm{H}^{2}$ \\
\hline $\operatorname{tm} 1(t d)$ & $<0.001$ & 0.81 & 0.002 & 0.77 \\
\hline$t 1(t d)$ & 0.002 & 0.75 & $<0.001$ & 0.9 \\
\hline$V x$ & $<0.001$ & 0.9 & $<0.001$ & 0.86 \\
\hline$t 2(t d)$ & $<0.001$ & 0.79 & 0.092 & 0 \\
\hline$t e(t d)$ & 0.002 & 0.76 & 0.001 & 0.78 \\
\hline $\mathrm{Cm}(\% \mathrm{CC} / \mathrm{td})$ & $<0.001$ & 0.81 & 0.001 & 0.79 \\
\hline$A P 1(\% C C . t d)$ & $<0.001$ & 0.81 & $<0.001$ & 0.85 \\
\hline$A P 2(\% C C . t d)$ & 0.076 & 0.00 & 0.131 & 0.00 \\
\hline$A P 3(\% C C . t d)$ & 0.007 & 0.7 & $<0.001$ & 0.81 \\
\hline$A \cup C(\% C C . t d)$ & $<0.001$ & 0.91 & $<0.001$ & 0.87 \\
\hline$t 2-t 1(t d)$ & 0.153 & 0.00 & 0.121 & 0.00 \\
\hline$t e-t 2(t d)$ & 0.012 & 0.66 & 0.032 & 0.6 \\
\hline TuWt $\left(\mathrm{g} / \mathrm{m}^{2}\right)$ & 0.073 & 0.00 & 0.001 & 0.78 \\
\hline $\mathrm{TuNr}\left(\mathrm{Tb} \# / \mathrm{m}^{2}\right)$ & 0.013 & 0.60 & $<0.001$ & 0.81 \\
\hline CC56DAP & 0.009 & 0.68 & 0.001 & 0.76 \\
\hline CC70DAP & 0.071 & 0.00 & 0.007 & 0.70 \\
\hline CC84DAP & 0.004 & 0.73 & $<0.001$ & 0.86 \\
\hline
\end{tabular}

\section{Correlations among traits}

The correlations of traits both under well-watered and drought stress conditions are given in Figure 3. Under both treatment conditions, maturity had a high correlation with AUC with intermediate maturity type genotypes generally having higher AUC than early ones. AUC reflects the amount of total intercepted light during the growing season of potato, and under well-watered and drought stress conditions AUC was correlated with tuber yield and tuber number. These correlations indicate that soil cover is an important trait in determining tuber yield under control as well as water-limited conditions. However, AUC correlation with tuber yield is higher under control (0.68) than water stress conditions $(0.44)$, which may suggest that the influence of this trait on tuber yield is less under drought stress conditions. Similarly, the correlation of AUC with tuber number was higher under well-watered than drought stress condition, suggesting that drought may directly affect formation of new tubers. 
The maximum soil cover attained $(\mathrm{V} x)$ under both treatment conditions had a positive correlation with tuber yield, tuber number and tuber dry weight, with higher values for control conditions. Vx had higher correlation value with AP1 (0.89 and 0.81 under control and stress conditions, respectively) than with AP2 and AP3. AP2 had the lowest correlation with $V x$, and under both treatment conditions the duration (t2-t1) was shorter that would affect AP2. Vx and the duration of each developmental phase of canopy determine the respective areas.

\begin{tabular}{|c|c|c|c|c|c|c|c|c|c|c|c|c|c|c|c|c|c|c|}
\hline & PM & AUC & Vx & $\mathrm{t} 2-\mathrm{t} 1$ & t1 & te & t2 & $\operatorname{tm} 1$ & te-t2 & AP3 & AP2 & AP1 & TuWt & TuNr & $\mathrm{Cm}$ & CC56DAP & 70DAP C & CC84DAP \\
\hline & 1.00 & 0.67 & 0.59 & 0.23 & 0.24 & 0.65 & 0.39 & 0.33 & 0.14 & 0.53 & 0.40 & 0.42 & 0.29 & 0.09 & 0.60 & -0.05 & -0.18 & -0.04 \\
\hline AUC & 0.64 & 0.67 & 0.96 & 0.11 & 0.58 & 0.67 & 0.58 & 0.46 & 0.00 & 0.66 & 0.43 & 0.85 & 0.68 & 0.58 & 0.65 & 0.03 & 0.15 & -0.03 \\
\hline Vx & 0.63 & 0.92 & 0.70 & -0.05 & 0.67 & 0.51 & 0.53 & 0.60 & -0.08 & 0.65 & 0.28 & 0.89 & 0.63 & 0.58 & 0.65 & 0.13 & 0.20 & -0.08 \\
\hline $\mathrm{t} 2-\mathrm{t} 1$ & -0.05 & -0.03 & -0.03 & 0.35 & -0.25 & -0.01 & 0.58 & -0.14 & -0.50 & -0.41 & 0.91 & -0.14 & -0.03 & -0.10 & 0.17 & -0.33 & -0.25 & -0.22 \\
\hline $\mathrm{t} 1$ & 0.34 & 0.66 & 0.63 & -0.28 & 0.35 & 0.23 & 0.64 & 0.77 & -0.37 & 0.17 & -0.04 & 0.88 & 0.40 & 0.48 & -0.04 & -0.09 & 0.20 & -0.26 \\
\hline te & 0.35 & 0.61 & 0.32 & -0.34 & 0.50 & 0.48 & 0.18 & 0.25 & 0.57 & 0.73 & 0.17 & 0.41 & 0.55 & 0.33 & 0.47 & 0.00 & 0.07 & 0.20 \\
\hline t2 & 0.25 & 0.53 & 0.50 & 0.59 & 0.61 & 0.14 & 0.11 & 0.53 & -0.70 & -0.18 & 0.69 & 0.63 & 0.31 & 0.33 & 0.10 & -0.30 & 0.00 & -0.36 \\
\hline $\operatorname{tm} 1$ & 0.36 & 0.53 & 0.50 & -0.25 & 0.74 & 0.64 & 0.42 & 0.51 & -0.26 & 0.23 & 0.02 & 0.58 & 0.27 & 0.26 & 0.26 & 0.06 & 0.21 & -0.30 \\
\hline te-t2 & 0.14 & 0.19 & -0.04 & -0.67 & 0.04 & 0.77 & -0.52 & 0.28 & 0.17 & 0.68 & -0.45 & -0.23 & 0.14 & -0.04 & 0.26 & 0.27 & 0.06 & 0.48 \\
\hline AP3 & 0.48 & 0.63 & 0.46 & -0.61 & 0.37 & 0.83 & -0.19 & 0.50 & 0.84 & 0.54 & -0.18 & 0.42 & 0.54 & 0.40 & 0.69 & 0.32 & 0.18 & 0.26 \\
\hline AP2 & 0.06 & 0.16 & 0.20 & 0.94 & -0.19 & -0.29 & 0.62 & -0.16 & -0.65 & -0.51 & 0.47 & 0.14 & 0.19 & 0.08 & 0.38 & -0.31 & -0.14 & -0.23 \\
\hline AP1 & 0.45 & 0.81 & 0.84 & -0.17 & 0.86 & 0.31 & 0.59 & 0.45 & -0.11 & 0.34 & -0.01 & 0.51 & 0.58 & 0.60 & 0.27 & -0.01 & 0.21 & -0.11 \\
\hline TuWt & 0.32 & 0.44 & 0.33 & -0.08 & 0.08 & 0.34 & 0.00 & -0.04 & 0.29 & 0.45 & -0.06 & 0.27 & 0.15 & 0.70 & 0.43 & 0.19 & 0.30 & 0.12 \\
\hline TuNr & 0.29 & 0.45 & 0.39 & -0.12 & 0.08 & 0.31 & -0.03 & 0.00 & 0.28 & 0.47 & -0.07 & 0.27 & 0.85 & 0.16 & 0.21 & 0.35 & 0.53 & 0.20 \\
\hline $\mathrm{Cm}$ & 0.37 & 0.31 & 0.42 & 0.21 & -0.31 & 0.01 & -0.09 & 0.09 & 0.06 & 0.24 & 0.39 & -0.11 & 0.14 & 0.28 & 0.51 & 0.24 & 0.06 & -0.03 \\
\hline CC56DAP & -0.22 & 0.05 & 0.03 & 0.17 & 0.07 & 0.11 & 0.19 & 0.12 & -0.01 & -0.04 & 0.19 & 0.00 & -0.17 & 0.16 & 0.04 & 0.53 & 0.66 & 0.71 \\
\hline CC70DAP & -0.10 & 0.23 & 0.29 & 0.28 & -0.16 & -0.13 & 0.09 & -0.11 & -0.14 & -0.01 & 0.38 & 0.03 & 0.05 & 0.41 & 0.48 & 0.86 & 0.83 & 0.62 \\
\hline CC84DAP & -0.32 & -0.04 & -0.04 & -0.39 & 0.11 & 0.26 & -0.27 & 0.30 & 0.32 & 0.28 & -0.44 & -0.03 & 0.23 & 0.30 & -0.07 & 0.27 & 0.26 & 0.35 \\
\hline
\end{tabular}

Figure 3. Heat map for correlation between canopy development curve parameters and yield traits under well-watered (upper triangle) and drought stress (lower triangle) conditions. The diagonal represents the correlation between well-watered and stress conditions for the different traits. The maturity score (PM) for the well-watered and drought was the same, taken from well-watered trials (Celis-Gamboa 2002).

Under stress conditions, AP1 had lower correlation with tuber yield and tuber number compared to AP3. However, this was not in line with what was observed under well-watered conditions, where AP1 had higher correlation with the yield traits. Similarly, parameter t1 had very low correlation (0.08) with tuber yield and tuber number under drought, in contrast to well-watered conditions where correlation value was 0.4 and 0.48 for each trait 
respectively. Furthermore, t2 had higher correlation with tuber yield traits under wellwatered conditions than water stress conditions. As expected, each area under the canopy curve had very high positive correlation with its respective duration phase (AP1 and t1, AP2 and $\mathrm{t} 2-\mathrm{t} 1, \mathrm{AP} 3$ and te-t2) under both treatment conditions. The correlation between chlorophyll content measured on 56, 70, and 84 DAP and most canopy curve parameters was low under both treatment conditions. However, under stress conditions there were positive correlations of CC70DAP with AP2 (0.38), tuber number (0.41), and $\mathrm{Cm}(0.48)$, while under well-watered conditions, CC70DAP had a positive correlation with tuber number (0.53) and CC84DAP with te-t2 (0.48). The correlation between the control and stress conditions is indicated in the diagonal of Figure 3, and showed strong differences for the different canopy curve parameter and traits. The lowest correlation between drought stress and well-watered conditions was observed for $\mathrm{t} 2$, TuNr, and TuWt.

\section{Discussion}

Drought stress has been recognized as one of the most important abiotic stresses in potato production (Monneveux et al. 2013; Levy et al. 2013). As a first sign of drought stress in the potato crop, reduction in leaf size is observed (Jefferies and MacKerron 1993), and reduction in leaf size and leaf expansion has been associated with a decreased canopy formation (Fleisher et al. 2008). In line with these studies, drought stress has resulted in reduced canopy development where AP1 and AP2 were reduced in response to drought stress. Area under the first phase of canopy development AP1 may indicate the effect of water shortage on leaf appearance and canopy expansion; moreover reduced AP2 and shorter t2-t1 under drought stress indicate the impact of drought stress on maximum canopy expansion and canopy maintenance. This has led to an earlier decline in soil cover (t2) under drought stress conditions as the result of drought induced senescence. Under well-watered conditions the decline in soil cover (t2) started on average at 32.8 thermal days after emergence while for drought stress it was at 24.4 thermal days (Table 2). This parameter was also influenced by maturity type (Figure 2), with early genotypes under both treatment conditions starting to senesce (t2) earlier than intermediate genotypes. Area under the curve (AUC) was greatly reduced in response to drought stress. The mean number of thermal days to complete the life cycle (te) for drought stressed plants was 42 , and 52 for control conditions. 
Several authors have indicated that there is a positive relationship between canopy development and tuber yield production under drought stress conditions (Jefferies and MacKerron 1993; Schittenhelm et al. 2006). In the current study we observed correlations between canopy curve parameters and tuber yield under water stress conditions, although the correlation value was lower than to what was observed under well-watered conditions. Total area under the curve $(A \cup C)$ had lower correlation with tuber yield under stress conditions compared to well-watered conditions. Under normal growing conditions, tuber yield is determined by the amount of light intercepted (Haverkort and Harris 1987; Struik et al. 1990). In a previous study (Jefferies and MacKerron 1987), reduction in intercepted radiation due to decreased canopy expansion was suggested as one of the main reasons for tuber yield reduction in response to drought. Under drought stress conditions, cumulative light interception depends not only on the ability to sustain canopy expansion, but also on the ability to maintain the canopy in order to avoid premature senescence (Jefferies and MacKerron 1993). This may depend on the ability of the plant to keep a higher level of relative leaf water content under water-limited conditions. Some studies (Chaves and Oliveira 2004; Blum 2011) have suggested that maintaining high relative water content under stress conditions promotes drought tolerance in plants and also helps in rapid recovery upon rehydration. Maintaining high relative water content might be possible through osmotic adjustment, where solutes are accumulated upon declining water potential in order to maintain leaf hydration and avoid cellular desiccation. Tuber yield under stress conditions is likely to also depend at least partially on partitioning of accumulated dry matter (Jefferies and MacKerron 1993). In addition to genotype and developmental differences, environmental factors such as drought can affect the partitioning of carbon at the leaf and whole plant levels (Chaves 1991).

In our trials, the strength of correlation observed for canopy curve parameters and tuber yield traits was not the same under drought stress and well-watered conditions. This may suggest that the strong link between canopy and tuber yield is weakened under waterlimiting conditions, and that other physiological adaptations may affect tuber yield in response to water shortage. Under well-watered conditions, most curve-fit parameters ( $V x$, AP1, AP3, AUC) had higher correlation with tuber yield and tuber number. These correlations indicates that there is close and positive relationship between canopy cover and tuber yield 
production in line with (Van Oijen 1991). Under stress conditions, AP1 had a higher correlation with $\mathrm{Vx}$ and $\mathrm{AUC}$ than AP2 and AP3, suggesting that the first developmental phase of canopy more strongly affected the total area under the curve. However, the level of correlation of AP1 with tuber yield and tuber number was lower compared to AP3. At this early developmental phase the canopy is characterized by the appearance of leaves and expansion of soil cover. Water shortage at an early growth stage can reduce radiation interception as a result of slower and less canopy expansion (Jefferies 1995a). Some authors (MacKerron and Jefferies 1986; Haverkort et al. 1990) reported that severe and prolonged drought stress at the early developmental stages can result in reduced tuber initiation and thereby reduce tuber yield. The physiology of tuber initiation involves biochemical and molecular signals that link photoperiod perception in leaves to changes in cellular growth patterns in stolons (Sarkar 2010). However, under non-optimal conditions such as drought stress or heat stress tuberization can be affected (Deblonde and Ledent 2001; Hancock et al. 2014) and Chapter 4 of this thesis). Tuberization is controlled by the potato Cycling DOF factor-1 (StCDF1) gene present on chromosome 5, which is also underlying the earliness locus (Kloosterman et al. 2013). Expression of this gene is regulated by binding of the photoperiodically controlled FKF-1 and GIGANTEA proteins, and StCDF1 appears to influence expression of the tuberisation signal StSP6A. The knowledge of the molecular mechanism of tuberization will help in investigating the molecular interaction drought stress with the tuber initiation process.

The effect of drought stress on potato depends on the timing as well as severity of stress applied (Monneveux et al. 2013). Drought stress applied at the stolonization stage may have a more pronounced effect on tuber number and yield than imposing stress at tuber initiation. The genotypes used in the current study have been selected from a previous drought experiment for having contrasting responses to drought (Chapter 3 of this thesis). In both experiments the same genotypes had a better tuber yield under drought stress conditions. However, drought had a more pronounced effect on number of tubers and tuber yield in the current study. This is likely due to the fact that in this study the genotypes were stressed at the stage of stolonization, with a more severe effect of drought stress on tuber initiation resulting in a reduction of tuber number and thus reducing tuber yield. Performing 
identical drought stress experiment under field conditions is very challenging due to uncontrollable environmental factors.

Chlorophyll content was indicated to have a close correlation with leaf photosynthetic capacity (Kato et al. 2004; Kumagai et al. 2009). However, the contribution of higher chlorophyll content (as pleasured by a SPAD meter) to yield under drought stress condition is under debate. Under drought stress conditions, increased chlorophyll content in potato has been associated either with reduced leaf growth (Rolando et al. 2015) or delayed senescence (Yactayo et al. 2013; Ramírez et al. 2014), depending on the developmental stage in which drought stress is imposed, the time of measurement and the developmental stage of the measured leaf. In our study, the increase in chlorophyll content could be a result of reduced leaf expansion that increased the leaf chlorophyll density. Drought stress treatment showed significant differences for chlorophyll content measured at 56 DAP (CC56DAP). However, chlorophyll content measured on 70 and 84 DAP (39.9 and 43.2 average thermal day respectively) under drought stress conditions did not show significant differences from control conditions. This may suggest that there is a significant effect of drought on leaf expansion (relatively early after exposure to stress). The absence of a significant effect of drought on chlorophyll content at later stages may be caused by the effect of senescence masking the effect of leaf expansion.

In summary, our results indicate that the relationship of canopy cover with tuber yield under drought stress conditions is not as strong as under well-watered conditions. The less strong link between canopy cover and tuber yield under water limitation may suggest that under these conditions, canopy cover-independent factors possibly affecting tuberization directly may play a role as well. This needs to be supported with further experiments that include a higher number of genotypes that would allow exploiting the genetic variation for canopy cover and identifying the genetic factors contributing to this variation. This would help to further understand what underlies the relationship between canopy cover and tuber yield under water limiting conditions. 


\section{References}

Allen E, Scott RK (1980) An analysis of growth of the potato crop. The Journal of Agricultural Science 94 (03):583-606

Blum A (2011) Drought resistance-is it really a complex trait? Functional Plant Biology 38 (10):753-757

Celis-Gamboa BC (2002) The life cycle of the potato (Solanum tuberosum L.): from crop physiology to genetics. PhD Thesis, Wageningen University, Wageningen. ,

Chaves M (1991) Effects of water deficits on carbon assimilation. Journal of experimental botany $42(1): 1-16$

Chaves M, Oliveira M (2004) Mechanisms underlying plant resilience to water deficits: prospects for water-saving agriculture. Journal of experimental botany 55 (407):23652384

Deblonde P, Ledent J-F (2001) Effects of moderate drought conditions on green leaf number, stem height, leaf length and tuber yield of potato cultivars. European Journal of Agronomy 14 (1):31-41

Fleisher DH, Timlin DJ, Reddy VR (2008) Interactive effects of carbon dioxide and water stress on potato canopy growth and development. Agronomy Journal 100 (3):711-719. doi:10.2134/agronj2007.0188

Hancock RD, Morris WL, Ducreux L, Morris JA, Usman M, Verrall SR, Fuller J, Simpson CG, Zhang R, Hedley PE (2014) Physiological, biochemical and molecular responses of the potato (Solanum tuberosum L.) plant to moderately elevated temperature. Plant, cell \& environment 37 (2):439-450

Haverkort A, Harris P (1987) A model for potato growth and yield under tropical highland conditions. Agricultural and forest meteorology 39 (4):271-282

Haverkort A, Uenk D, Veroude H, Van de Waart M (1991) Relationships between ground cover, intercepted solar radiation, leaf area index and infrared reflectance of potato crops. Potato Research 34 (1):113-121

Haverkort A, Van de Waart M, Bodlaender Kd (1990) The effect of early drought stress on numbers of tubers and stolons of potato in controlled and field conditions. Potato Research 33 (1):89-96

Iwama K (2008) Physiology of the Potato: New Insights into Root System and Repercussions for Crop Management. Potato Research 51 (3-4):333-353. doi:10.1007/s11540-0089120-3

Jefferies R (1995a) Physiology of crop response to drought. In: Potato ecology and modelling of crops under conditions limiting growth. Springer, pp 61-74

Jefferies R, MacKerron D (1987) Aspects of the physiological basis of cultivar differences in yield of potato under droughted and irrigated conditions. Potato Research 30 (2):201217

Jefferies R, MacKerron D (1993) Responses of potato genotypes to drought. II. Leaf area index, growth and yield. Ann Appl Biol 122 (1):105-112

Jefferies RA (1995b) Physiological determinants of genotypic differences in carbon isotope discrimination in potato grown in well-watered conditions. Ann Appl Biol 127 (3):585592

Kato M, Kobayashi K, Ogiso E, Yokoo M (2004) Photosynthesis and dry-matter production during ripening stage in a female-sterile line of rice. Plant production science 7 (2):184188 
Khan MS (2012) Assessing genetic variation in growth and development of potato. PhD Thesis, Wageningen University, Wageningen

Khan MS, van Eck HJ, Struik PC (2013) Model-based evaluation of maturity type of potato using a diverse set of standard cultivars and a segregating diploid population. Potato Research 56 (2):127-146

Kloosterman B, Abelenda JA, Gomez MdMC, Oortwijn M, de Boer JM, Kowitwanich K, Horvath BM, van Eck HJ, Smaczniak C, Prat S, Visser RGF, Bachem CWB (2013) Naturally occurring allele diversity allows potato cultivation in northern latitudes. Nature 495 (7440):246-250

Kumagai E, Araki A, Kubota $F$ (2009) Correlation of chlorophyll meter readings with gas exchange and chlorophyll fluorescence in flag leaves of rice (Oryza sativa L.) plants. Plant production science 12 (1):50-53

Lahlou O, Ouattar S, Ledent J-Fo (2003) The effect of drought and cultivar on growth parameters, yield

and yield components of potato. Agronomie 23 (3):257-268. doi:10.1051/agro:2002089

Levy D, Coleman WK, Veilleux RE (2013) Adaptation of potato to water shortage: irrigation management and enhancement of tolerance to drought and salinity. American Journal of Potato Research 90 (2):186-206

Mackerron D, Jefferies R (1986) The influence of early soil moisture stress on tuber numbers in potato. Potato Research 29 (3):299-312

Martin $\mathrm{R}$ The effect of nitrogen fertilizer on the recovery of nitrogen by a potato crop. In: Proceedings of Agronomy Society of New Zealand, 1995. pp 97-104

Monneveux P, Ramirez DA, Pino MT (2013) Drought tolerance in potato (S. tuberosum L.) Can we learn from drought tolerance research in cereals? Plant Science 205:76-86. doi:10.1016/j.plantsci.2013.01.011

Ospina C, van Bueren EL, Allefs J, Engel B, van der Putten P, van der Linden C, Struik P (2014) Diversity of crop development traits and nitrogen use efficiency among potato cultivars grown under contrasting nitrogen regimes. Euphytica 199 (1-2):13-29

Ospina CA (2016) Nitrogen use efficiency in potato: an integrated agronomic, physiological and genetic approach. PhD Thesis, Wageningen University, Wageningen,

Ramírez D, Yactayo W, Gutiérrez R, Mares V, De Mendiburu F, Posadas A, Quiroz R (2014) Chlorophyll concentration in leaves is an indicator of potato tuber yield in watershortage conditions. Scientia Horticulturae 168:202-209

Rolando JL, Ramírez DA, Yactayo W, Monneveux P, Quiroz R (2015) Leaf greenness as a drought tolerance related trait in potato (Solanum tuberosum L.). Environmental and Experimental Botany 110:27-35

Sarkar D (2010) Photoperiodic inhibition of potato tuberization: an update. Plant growth regulation 62 (2):117-125

Schittenhelm S, Sourell H, Löpmeier F-J (2006) Drought resistance of potato cultivars with contrasting canopy architecture. European Journal of Agronomy 24 (3):193-202. doi:10.1016/j.eja.2005.05.004

Struik PC, Haverkort A, Vreugdenhil D, Bus C, Dankert R (1990) Manipulation of tuber-size distribution of a potato crop. Potato Research 33 (4):417-432

Van Oijen M (1991) Light use efficiencies of potato cultivars with late blight (Phytophthora infestans). Potato research 34 (2):123-132

Van Ooijen J (2006) JoinMap 4. Software for the calculation of genetic linkage maps in experimental populations Kyazma BV, Wageningen, Netherlands 
Van Ooijen J (2009) MapQTL 6. Software for the mapping of quantitative trait loci in experimental populations of diploid species Kyazma BV: Wageningen, Netherlands

Yactayo W, Ramírez DA, Gutiérrez R, Mares V, Posadas A, Quiroz R (2013) Effect of partial root-zone drying irrigation timing on potato tuber yield and water use efficiency. Agricultural Water Management 123:65-70

Yin X, Goudriaan J, Lantinga EA, Vos J, Spiertz HJ (2003) A flexible sigmoid function of determinate growth. Annals of botany 91 (3):361-371

\section{Supplementary file}

Table S1. List of CxE dipoloid potato mapping population used in the current study with their maturity class, tuber number and yield under drought stress conditions.

\begin{tabular}{llcrrr}
\hline \multirow{2}{*}{ Genotypes } & \multirow{2}{*}{ Maturity } & \multicolumn{2}{c}{ Tuber number } & \multicolumn{2}{c}{ Tuber yield } \\
& & per plant & per $\mathrm{m}^{2}$ & $\mathrm{~g} /$ plant & $\mathrm{g} / \mathrm{m}^{2}$ \\
\hline CE250 & Early & 8 & 35.6 & 47 & 208.9 \\
CE159 & Early & 20 & 88.9 & 62 & 275.6 \\
CE747 & Early & 11 & 48.9 & 68 & 302.2 \\
CE027 & Early & 6 & 26.7 & 75 & 333.3 \\
CE605 & Early & 14 & 62.2 & 101 & 448.9 \\
CE639 & Early & 10 & 44.4 & 141 & 626.7 \\
CE633 & Early & 11 & 48.9 & 174 & 773.3 \\
CE736 & Early & 9 & 40.0 & 193 & 857.8 \\
CE685 & Early & 24 & 106.7 & 245 & 1088.9 \\
CE195 & Intermediate & 8 & 35.6 & 51 & 226.7 \\
CE603 & Intermediate & 11 & 48.9 & 106 & 471.1 \\
CE017 & Intermediate & 16 & 71.1 & 110 & 488.9 \\
CE277 & Intermediate & 24 & 106.7 & 136 & 604.4 \\
CE668 & Intermediate & 15 & 66.7 & 145 & 644.4 \\
CE688 & Intermediate & 14 & 62.2 & 166 & 737.8 \\
CE110 & Intermediate & 27 & 120.0 & 183 & 813.3 \\
CE738 & Intermediate & 11 & 48.9 & 236 & 1048.9 \\
CE719 & Intermediate & 20 & 88.9 & 311 & 1382.2 \\
CE653 & Intermediate & 15 & 66.7 & 389 & 1728.9 \\
\hline
\end{tabular}



Chapter 6

General discussion 



\section{Introduction}

Global agriculture is facing a serious threat as resources such as water are becoming very scarce (Chaves et al. 2003). It is predicted that with the change in climate in the coming decades drought will escalate (Godfray et al. 2010). The negative consequences of climate change on agriculture indicate the need to develop climate resilient crops. In order to achieve this, a better understanding of drought tolerance in plants from molecular, physiological and morphological perspectives is required. There are many studies done in cereal crops to understand drought responses (Fleury et al. 2010). These studies have contributed to breeding crops that better deal with drought stress conditions (Ashraf 2010).

Potato is recognized as an efficient water user compared to the most widely grown crops (wheat, rice and maize) in the world. However, several authors have pointed out the sensitivity of potato to even moderate water shortage which has a significant effect on tuber yield (Deblonde and Ledent 2001; Iwama 2008; Anithakumari et al. 2012). The ever-changing climate will affect potato production in complex and location-dependent ways. In temperate climate potatoes are grown during the rainy summer season and dry spells during various growth stages may lead to transient water shortage and drought stress (Levy et al. 2013). Potato production in semi-arid and arid regions depends on irrigation, and water shortage in these regions is a common phenomenon. This calls for drought tolerance breeding programs that can improve tuber yield under water limited conditions.

Many of the drought tolerance studies in potato have focused on selecting drought tolerant varieties rather than dissecting into traits that contribute to drought tolerance in potato (Monneveux et al. 2013). However, there are few studies done in dissecting drought tolerance traits in potato (Anithakumari et al. 2011; Anithakumari et al. 2012; Khan et al. 2015). Understanding the physiology and genetic basis of drought tolerance in potato helps in designing breeding programs for enhanced drought tolerance. This thesis aimed at a better understanding of drought tolerance in potato in response to prolonged moderate and severe water stress as well as dissection of the genetic basis controlling drought tolerance traits. 
Drought tolerance mechanisms in potato involve drought escape, avoidance and tolerance (Obidiegwu et al. 2015). Drought escape usually happens when water shortage occurs at the later developmental stages, however this comes with a yield penalty as it involves early completion of the life cycle (Levy et al. 2013). In this thesis, we have conducted trials with mild and severe drought stress imposed at the early developmental stage of potato under greenhouse and field conditions, respectively. The field drought stress experiments were conducted in central Ethiopia, which is characterized by a semi-arid climate. The response of potato to a drought stress that occurs at an early developmental stage involved reduction in shoot and root biomass, and in tuber yield production. Genotypes with better tuber yield production under severe and prolonged drought stress conditions had a positive correlation of tuber yield with shoot biomass produced, suggesting drought avoidance as tolerance mechanism. Tolerant genotypes under drought stress conditions were able to keep higher shoot biomass than susceptible ones, which indicates they were able to keep growing under water limited conditions. According to Blum (1998), drought avoidance refers to a plant's ability to retain a relatively high level of hydration under drought stress conditions which can be achieved either through enhanced water uptake or reduced water loss. Increased level of water uptake requires adaptation of root morphology that includes root thickness, increased root length and mass. However in the current study root length and root mass (both fresh and dry) under drought stress conditions were not higher than under well-watered conditions and we did not measure root thickness. Decreased water loss can involve reduced epidermal conductance and reduced leaf area which helps to minimize water loss through transpiration. Although we did not measure these traits, chlorophyll content measured under drought stress conditions was indicative for reduced leaf area, as higher chlorophyll content levels were recorded for drought stress condition than under well-watered conditions. This typically is an indication for reduced leaf expansion, resulting in reduced leaf area. The use of chlorophyll content as an indicator of reduced leaf area under drought stress conditions is discussed in detail later.

\section{The complexity of drought tolerance}

Drought tolerance is a quantitative trait with complex phenotypic and genetic control (Tuberosa 2012). In a natural environment, drought stress may not occur alone, but together with other abiotic and biotic stresses adding to the complexity of drought tolerance. For this 
reason research towards understanding the molecular and genetic basis of drought tolerance in plants is crucial. The progress in breeding efforts to develop better yielding crops under water limited conditions is hampered by the quantitative genetic basis of drought tolerance (Passioura 2002). The slow progress in improving tuber yield production in potato under water limited conditions may reflect the complex genetics of drought tolerance (Anithakumari et al. 2012; Monneveux et al. 2013). It has been indicated that heritability of tuber yield under water stress conditions is usually low (Cabello et al. 2014), which would also explain the slow progress of yield enhancement for stress conditions. However, heritability ranged from moderate to high in our studies (Chapters 3 and 4). The differences in heritability estimates could be the result of difference in environmental variances, number of genotypes used or accuracy of measurement in addition to trait heritability. In our research we used a larger number of genotypes compared to studies by Cabello et al. (2014). Besides, moderate to high heritability for drought tolerance related traits and tuber yield was found to be in line with other recent drought tolerance studies done both in greenhouse and field (Anithakumari et al. 2012; Khan et al. 2015). It is also important to account for the developmental stage in which stress has occurred and the level of stress severity in breeding potato for an improved drought tolerance. The response of potato to mild (greenhouse; Chapter 4) and severe stress (field; Chapter 3) imposed at different developmental stages is summarized in Figure 1. 


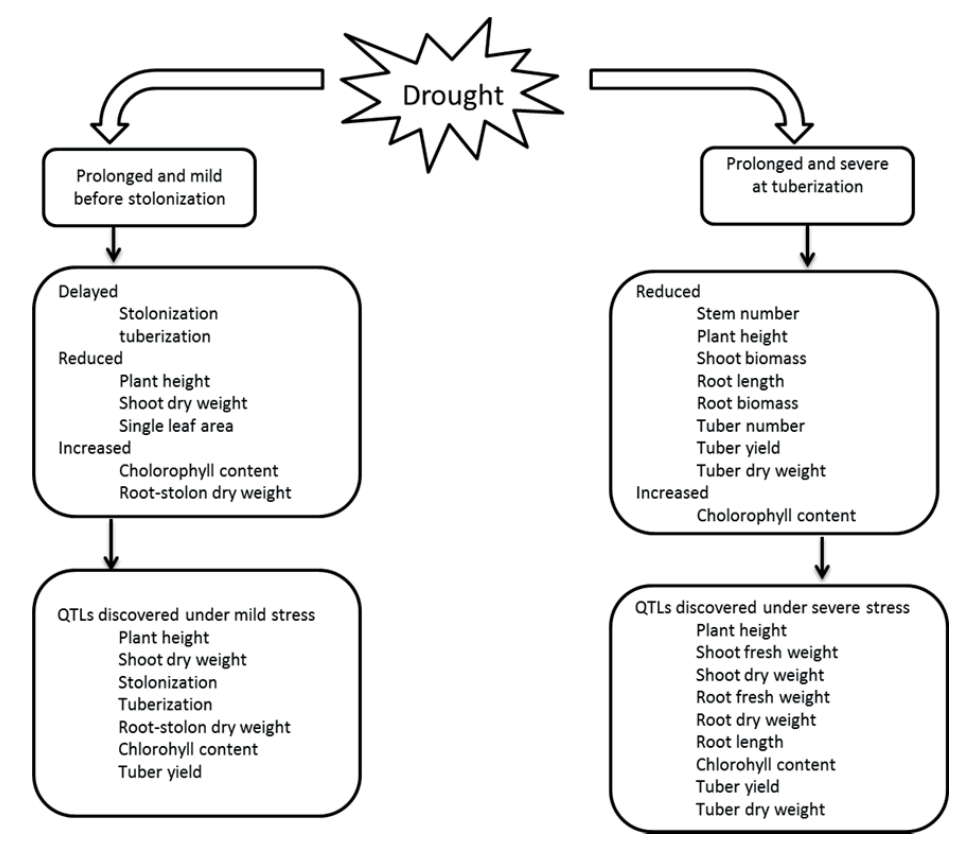

Figure 1. Flow chart showing the effect of prolonged mild (Greenhouse; Chapter 4) and severe drought (field; Chapter 3 ) stress on potato growth and how potato responds at the morphological level. QTLs discovered under mild and drought stress controlling drought tolerance traits are indicated.

\section{Dissecting complex traits}

Genetically dissecting complex traits has been made possible with the application of QTL analysis and association mapping. QTL mapping has been used in the last two decades to dissect traits related to drought tolerance mostly in cereals and it is reviewed in (Ashraf 2010). These studies have demonstrated the power of using QTL studies to understand the genetic basis controlling physiological and morphological responses in drought-stressed plants. this has led to identification of QTL for drought tolerance related traits like osmotic adjustment (Robin et al. 2003; Teulat et al. 1998), isotope discrimination (Anithakumari et al. 2012), root characteristics (Courtois et al. 2009), and delayed senescence (stay green) 
(Harris et al. 2007). Recently, several studies reported on the use of QTL analysis to dissect the genetic basis of drought tolerance in potato, and these studies have identified QTLS linked to physiological and morphological traits related to drought stress (Anithakumari et al. 2011; Anithakumari et al. 2012; Khan et al. 2015). These studies were done under in vitro, green house and field conditions.

The dissection of many complex traits such as drought can be maximized by using association mapping which allows finding significant QTLs associated with traits of interest in relatively unrelated genotypes and the higher number of recombinations result in a higher mapping resolution (Zhu et al. 2008). Association mapping has been used to dissect complex traits such as drought tolerance in a number of different crops including barley (Varshney et al. 2012), maize (Xue et al. 2013) and salt tolerance in barley (Long et al. 2013) and rice (Kumar et al. 2015).Several studies using association mapping analysis in potato were performed for traits other than drought tolerance (Gebhardt et al. 2004; Simko 2004; D'hoop et al. 2008; Ospina 2016).These studies have demonstrated the power of association mapping in potato for discovering significant SNPs associated with different kind of traits.

Drought tolerance in potato is not explained by a single trait; rather tuber yield under water limiting conditions is determined by the aggregated effects of morphological and physiological traits. In order to find drought tolerance traits and the genetic basis that control these traits, we have evaluated the CXE diploid potato population for drought tolerance under field conditions (Chapter 3). Genotypes under field conditions were exposed to water limitation stress starting from tuber initiation and several morphological, physiological and yield traits were collected during the stress period. We were able to identify several QTLs linked to drought tolerance. These findings were also in line with QTLS reported earlier in the same population by (Anithakumari et al. 2012). Also, in Chapter 4, we have examined the effect of moderate water stress on tuber yield production of tetraploid potato cultivars under greenhouse conditions using association mapping. These modern potato cultivars were part of a cultivar set studied previously for quality traits using genome wide association mapping (D'hoop et al. 2008). 
In our drought experiment, water application was reduced to $50-60 \%$ of the optimum amount after two weeks of emergence. The water limited conditions delayed the stolon growth and tuber formation with a consequence of reduced final tuber yield production. Under well-watered and water-limited conditions, we identified significant SNPs associated with different measured traits. The response to drought stress under both severe and moderate drought stress was highly influenced by maturity type and most of the QTLS discovered co-localized with a maturity locus found on chromosome 5. However, QTLS located on different chromosomes other than chromosome 5 were also discovered under both stress conditions.

In Chapter 2, we used multi-trait QTL analysis to dissect the complex developmental processes in potato using CxE potato genotypes under well-watered field conditions only. The outcome from this Chapter is discussed in detail later. The experiments for Chapter 2 and 3 were both conducted in Ethiopia using the CxE diploid potato mapping population; however, there were environmental differences mainly in terms of temperature, soil type (clay loam vs light clay) and field management (irrigation vs rain-fed). The average temperature for Holetta (Chapter 2) was $130 \mathrm{C}$ while for Melkassa (Chapter 3) it was 280C. The environmental differences were reflected in the onset of senescence: senescence started 65 days after planting (DAP) in Melkassa and 80 DAP in Holetta. However, under both environmental conditions maturity was controlled by the same QTL locus located on chromosome 5.

\section{QTL by environment interaction}

The CXE genotypes used to dissect drought tolerance under field conditions in Chapter 3 were previously used in drought tolerance studies under in vitro (Anithakumari et al. 2011) and greenhouse conditions (Anithakumari et al. 2012). In the three (in vitro, greenhouse, field) different environments QTLs for growth traits and tuber yield were discovered under well-watered, drought stress and recovery conditions. The respective number of QTLS discovered were; 23 QTLs (in vitro), 47 QTLs (greenhouse), and 60 QTLs (field; Chapter 3). However, only some of the QTLs discovered in the three different environments for different traits overlapped. This limited overlap may be attributed to the environmental differences such as temperature, relative humidity, soil composition, growing medium etc. 
The interaction of QTL by environment (greenhouse and field) was seen by the variations of QTLs detected on chromosome 5, where under greenhouse conditions QTLs detected were predominantly for stress and recovery (Anithakumari et al. 2012), while under field conditions QTLs were detected for both drought stress and control conditions (Chapter 3). This was also observed in the mild drought stress experiment in the greenhouse (Chapter 4), where most of the significant marker trait associations were detected under water-limited conditions. Such differences in QTL detection indicates genotype by environment interactions which confirm that these quantitative traits depend on the cumulative action of many genes and their interaction with the environment, and suggests that depending on the growing conditions there are differences in the genetic control of the traits. Many studies have reported differences in QTL expressed as a result of QTL by environment interactions for different traits including drought tolerance traits (Khan et al. 2015), developmental traits (Hurtado et al. 2012) and nitrogen use efficiency (Ospina 2016). Thus, in order to produce suitable drought stress tolerant genotypes for multiple environments, we suggest multienvironment QTL studies combined with careful monitoring of the environment (including at least temperature, humidity, soil water potential and taking into account soil type differences).

In another drought tolerance experiment conducted under greenhouse and field conditions using a different potato mapping population, environment-specific QTLs were reported for drought tolerance traits (Khan et al. 2015). This indicates that it is important to consider differences in environmental conditions when interpreting results from drought tolerance experiments done under controlled and field conditions. Results from drought stress experiments conducted under field conditions may have direct relevance, as it represents the real growing conditions. However, genotype by environment interactions across different years and locations can complicate analysis and interpretation, as stated above since environmental factors such as temperature and day length can affect expression of QTLs controlling growth traits. Stable QTLs across years discovered under field conditions can be directly used to improve potato for drought tolerance, while environment-specific QTLS should be carefully used in the context of the matching target environment. 


\section{QTLs under severe and moderate drought stress}

Despite the differences in QTLs found among the different environments, most of the QTLS detected for growth traits on chromosome 5 under water stress conditions in the greenhouse overlapped with QTLs found under water-limited conditions in the field. This may suggest that the locus located on chromosome 5 has a pleiotropic effect, affecting earliness and controlling different other traits expressed under drought conditions. The allelic variation underlying earliness in potato has been elucidated, and is attributed to the Cycling DOF factor (CDF) 1 gene (Kloosterman et al. 2013). Overlap between QTLs detected under greenhouse (Anithakumari et al. 2012) and field conditions on chromosomes other than chromosome 5 are summarized in Table 1.

The co-localization of QTL for drought tolerance traits detected under severe (field) and mild (greenhouse) drought stress conditions on a specific region of chromosome 5 indicates that this genomic region influences traits measured under both stress levels. It has also been reported that this region influences drought tolerance traits under severe stress conditions in an experiment conducted in the greenhouse (Anithakumari et al. 2012), suggesting that there is an overlap of genomic regions that control the drought response in potato under prolonged mild and severe drought stress. The overlap of QTLs under different stress levels presents a great opportunity for improving potato for enhanced drought tolerance. However, most of the QTLs that showed an overlap under different levels of drought stress were influenced by maturity, therefore we suggest further experiments with a larger genotype set that would allow fine mapping of the maturity QTL region that accumulated QTLs of drought tolerance traits. This may give an answer to the question whether drought QTLs detected on chromosome 5 are all under the control of the maturity locus, or whether some QTLs are independent of maturity.

In the field drought stress experiment (Chapter 3), we also detected a QTL region adjacent to the maturity locus on chromosome 5 controlling harvest index calculated from dry weight measured under well-watered conditions. This may suggest the existence of two independent QTLs on chromosome 5 controlling growth and yield traits in potato under both control and water stress conditions. The presence of two independent QTL loci on chromosome 5 is supported by the discovery of an expression QTL (eQTL) hotspot in close 
proximity to the StCDF1 maturity locus that is only present under drought conditions, and appears to be a major switch for the drought response in potato (Anithakumari 2011; Muijen et al. 2016). Further analysis of this eQTL using the maturity locus as a covariate showed the eQTL hotspot to still be significant (Muijen et al. 2016). However, it may need further investigation to confirm the presence of two independent loci on chromosome 5 controlling drought tolerance through fine mapping of this region with a larger set of genotypes segregating for this locus. This may also help to differentiate between QTLs controlling earliness and drought tolerance traits. The CDF1 gene underlying the maturity locus is photoperiodically controlled, and is an important mediator of the photoperiodic control of tuberization (Kloosterman et al. 2013). The discovery of allelic variation underlying the CDF locus helped to better understand the tuber initiation process. CONSTANS genes that affect tuberization under long days (Kloosterman et al. 2013) were reported to be influenced by drought stress (Muijen et al. 2016). This may suggest that genes involved in regulation of tuberization in potato are directly affected by drought stress, and may thus influence tuber formation and therefore yield under water-limiting conditions. Furthermore, in the analysis of the gene regulatory network underlying the drought stress response in the CXE potato population, Nuclear factor y subunit C4 located on chromosome 5 (eQTL hotspot) was reported to be a key candidate to regulate the drought response, and to be part of the regulatory cascade that is involved in the Abscisic acid (ABA) signaling pathway (Muijen et al. 2016). The production of $A B A$ in response to drought stress serves as an early stress signal to the plant, regulating transpiration. Moreover, high expression of the environment-stress inducible gene TAS14 in drought-stressed genotypes was positively correlated with recovery after drought. The TAS14 gene was characterized in tomato (Godoy et al. 1994) and the overexpression of this gene was associated with increased drought tolerance, with higher biomass accumulation and better rehydration (Munoz-Mayor et al. 2012). This shows that understanding the gene regulatory system underlying the drought responses in combination with phenotypic QTL analysis can help in identification of candidate genes for drought tolerance.

For a better understanding of drought tolerance, many authors have studied drought related traits and their relationship with tuber yield (Tourneux et al. 2003; Lahlou and Ledent 2005; Monneveux et al. 2013). Traits related to drought tolerance to be used in marker assisted 
breeding programs are desirable to have high heritability, be genetically associated with yield under stress and easy to measure (Obidiegwu et al. 2015). As discussed earlier drought tolerance traits from our research had met these criteria, showing moderate to high heritability and correlation with tuber yield under drought conditions. The genetic studies on drought tolerance in potato in Chapter 3 and 4 have revealed that many growth traits contributed to drought tolerance in potato. However, the amount of phenotypic variation explained by the QTLs associated with different traits is variable. The phenotypic variance explained ranged from 28 to $54 \%$ for QTLs identified under severe drought stress conditions and $13-36 \%$ of variance was explained by QTLs found under mild drought stress. This information may help in prioritizing QTLs to be used in improving potato for drought tolerance in relation to the target environment and the expected drought pattern in this environment.

Morphological or physiological traits that have correlation with tuber yield production under drought stress conditions can be used as indirect drought tolerance selection criteria. In Chapter 3, we discovered QTLs for root length, root fresh weight and root dry weight that co-located on chromosome 5 with QTLs for plant height, shoot fresh and dry weight and all these traits had correlation with tuber yield under stress conditions. The phenotypic variances explained were 30,37 and $45 \%$ for root length, root fresh weight, and root dry weight respectively, while QTL detected for plant height, shoot fresh and dry weight explained 33,52 , and $50 \%$ of observed phenotypic variation, respectively. The co-location of QTLs controlling above and below ground traits helps to understand the link between them and how it relates to tuber yield under water limited conditions as tuber yield under drought stress conditions is determined by the aggregated effect of morphological and physiological traits. The amount of shoots maintained under drought stress conditions determines the amount of assimilates to be produced which in turn affect tuber bulking. In Chapter 5, we have observed that there is positive correlation between canopy cover and tuber yield under water limited conditions. Similarly, other drought tolerance studies (Anithakumari et al. 2012; Khan et al. 2015) have reported QTLs for root and shoot traits co-localizing on chromosome 5. We also detected drought-specific QTLs under severe drought stress conditions located on chromosome 7, 9 and 12 for tuber yield and on chromosome 8 for plant height. However, these QTLs were not reported from the greenhouse experiment of 
(Anithakumari et al. 2012). Although both experiments used a similar set of the genotypes, the two environments were quite different; recorded maximum temperature for greenhouse was 35.5 while it was 27.4 for the field conditions. Similarly, relative humidity was higher for the green house (65.6) than field (58.8). Besides, pots filled with soil were used as growing media in the greenhouse, restricting space for roots, stolons and tubers, while this was not limited for those planted in the field. These environmental differences could affect trait QTL expression as quantitative traits are highly influenced by the growing conditions. QTLS colocated under different environmental conditions, such as greenhouse and field can give a better clue of which genomic regions control drought tolerance traits under both conditions and are less environment-dependent.

In Table 1 QTLs co-located on the same location from three studies: greenhouse (Anithakumari et al. 2012), and field (Chapter 2; Hurtado-Lopez et al. 2015), (Chapter 3) are summarized. A QTL identified for tuber yield on chromosome 12 under drought stress conditions (field) was found to co-locate with QTLs controlling the onset and inflection point (a time point half way of the developmental process) of plant height under well-watered conditions using a single trait QTL analysis (Chapter 2). Other co-located QTLs include a QTL identified on chromosome 8 for plant height (Chapter 3) and a QTL for the inflection point of senescence, a parameter which indicates the time point half way of this developmental process, under well-watered conditions (Chapter 2; Single trait QTL analysis). Both experiments (Chapter 2 and 3) were conducted in Ethiopia, in an area characterized by different environmental factors that include at least water availability, temperature and soil as discussed earlier in this discussion. The co-location of these QTLs under different environmental conditions suggests that QTL located on chromosome 8 is expressed under a wide range of environments.

On chromosome 9 a QTL for tuber yield was detected that explained $9 \%$ of the phenotypic variation observed. On this same location QTLs for shoot fresh weight and $\delta 13 \mathrm{C}$ were reported explaining 24 and $12 \%$ of observed variation respectively (Table 1), suggesting a possible functional relationship between $\delta 13 \mathrm{C}$ and yield. $\delta 13 \mathrm{C}$ is an important trait linked with water use efficiency and can be used as a drought tolerance indicator (Levy et al. 2013), and is discussed in more detail later. An overlap of QTLs linked to different kinds of traits 
being detected in different environmental setups help to better understand genetic controls linked to the different kinds of traits and aid marker assisted breeding for drought tolerance.

Table 1. Overlap between QTLs detected for a variety of traits measured under well-watered (WW) and drought stress (DS) conditions on chromosomes other than chromosome 5 in the CxE diploid mapping population in two different environments, greenhouse and field.

\begin{tabular}{|c|c|c|c|c|c|c|}
\hline Chr & Traits & $\begin{array}{c}\text { Treatmen } \\
\mathrm{t}\end{array}$ & $\begin{array}{l}\text { Environmen } \\
\mathrm{t}\end{array}$ & Interval & $\begin{array}{c}\text { variance } \\
\text { explaine } \\
\mathrm{d}\end{array}$ & References \\
\hline \multirow[t]{2}{*}{1} & $\begin{array}{l}\text { chlorophyll } \\
\text { fluorescence }\end{array}$ & DS & Greenhouse & $54-63$ & 19.7 & Anithakumari et al. 2012 \\
\hline & CC34DAS & WW & Field & $51-59$ & 14.4 & Chapter 3 \\
\hline \multirow[t]{6}{*}{2} & Plant height & DS & Greenhouse & $77-102$ & 21.9 & Anithakumari et al. 2012 \\
\hline & CC3DAS & DS & Greenhouse & $89-102$ & 16.4 & Anithakumari et al. 2012 \\
\hline & CC19DAS & WW & Field & $83-101$ & 18.7 & Chapter 3 \\
\hline & CC29DAS & WW & Field & $90-104$ & 20.2 & Chapter 3 \\
\hline & CC34DAS & WW & Field & $92-103$ & 27.7 & Chapter 3 \\
\hline & CC34DAS & DS & Field & $87-100$ & 18.4 & Chapter 3 \\
\hline \multirow[t]{2}{*}{6} & $\begin{array}{l}\text { Root dry } \\
\text { weight }\end{array}$ & DS & Greenhouse & $77-87$ & 17.8 & Anithakumari et al. 2012 \\
\hline & Plant height & WW & Field & $71-77$ & 14.7 & Chapter 3 \\
\hline \multirow[t]{2}{*}{8} & Plant height & WW & Field & $1-18.6$ & 13.7 & Chapter 3 \\
\hline & $\begin{array}{l}\text { Senescence } \\
\text { (inflection } \\
\text { point) }\end{array}$ & WW & Field & 11.5 & 4.1 & $\begin{array}{l}\text { Chapter } 2 \text { (Hurtado-Lopez et } \\
\text { al. 2015) }\end{array}$ \\
\hline \multirow[t]{3}{*}{9} & $\begin{array}{l}\text { Shoot fresh } \\
\text { weight }\end{array}$ & DS & Greenhouse & $26-48$ & 24.6 & Anithakumari et al. 2012 \\
\hline & $\delta 13 C$ & DS & Greenhouse & $34-40$ & 12.7 & Anithakumari et al. 2012 \\
\hline & $\begin{array}{l}\text { Tuber fresh } \\
\text { weight }\end{array}$ & DS & Field & $27-35$ & 9 & Chapter 3 \\
\hline \multirow[t]{3}{*}{10} & $\delta 13 C$ & WW & Greenhouse & $63-74$ & 22.8 & Anithakumari et al. 2012 \\
\hline & CC3DAS & DS & Greenhouse & $63-75$ & 15.4 & Anithakumari et al. 2012 \\
\hline & CC19DAS & DS & Field & $66-73$ & 21.2 & Chapter 3 \\
\hline \multirow[t]{3}{*}{12} & $\begin{array}{l}\text { Tuber fresh } \\
\text { weight }\end{array}$ & DS & Field & $1-21.6$ & 9.4 & Chapter 3 \\
\hline & $\begin{array}{l}\text { Plant height } \\
\text { (onset) }\end{array}$ & WW & Field & 16.5 & 7.7 & $\begin{array}{l}\text { Chapter } 2 \text { (Hurtado-Lopez et } \\
\text { al. 2015) }\end{array}$ \\
\hline & $\begin{array}{l}\text { Plant height } \\
\text { (Inflection } \\
\text { point) }\end{array}$ & WW & Field & 16.5 & 6.1 & $\begin{array}{l}\text { Chapter } 2 \text { (Hurtado-Lopez et } \\
\text { al. 2015) }\end{array}$ \\
\hline
\end{tabular}


Under water stress conditions, secondary traits such as photosynthetic rate can be indicative for yield. We have identified QTLs associated with chlorophyll content under stress conditions (Chapter 3 and 4). Increased chlorophyll content under drought stress conditions reflects the ability of a plant to maintain greenness (stay green) under water limiting conditions and in sorghum stay green is linked to better yield under drought stress conditions (Harris et al. 2007). Such a trait can be indicative for yield under stress conditions. As mentioned before, contributing traits to be used as drought tolerance indicators should be highly heritable, easy to measure, stable within the measurement period and correlated with yield (Prasanna et al. 2013). In potato increased chlorophyll content (greenness) under drought stress conditions can either be associated with reduction in leaf growth (Rolando et al. 2015) or delayed senescence (Yactayo et al. 2013; Ramírez et al. 2014). This may depend on the time and level of stress applied, and the timing of the measurements. Therefore, it is important to consider the developmental stage in which chlorophyll content is measured, the timing and level of stress imposed in order to consider increased leaf greenness as an indicator of delayed senescence (Rolando et al. 2015). Several of the QTLs detected for increased chlorophyll content under stress conditions in Chapter 3 and 4 could either indicate reduction in leaf size or delayed senescence. There was co-localization of QTL for chlorophyll content measured at 34DAS with a QTL detected for plant height under stress conditions (Table 1). On chromosome 1, we have also observed co-localization of a QTL for CC34DAS measured under control conditions with a QTL for chlorophyll fluorescence measured under stress conditions (Table 1). On chromosome 10 a QTL for chlorophyll content measured under drought stress conditions was detected and co-located with $\delta 13 \mathrm{C}$ measured under well-watered conditions in the greenhouse (Table 1). Carbon isotope discrimination is associated with water use efficiency (WUE) in potato (Vos and Groenwold 1989). On chromosome 10 we also identified a significant marker trait association for tuber yield under well-watered conditions and this QTL overlapped with the QTL reported for carbon isotope discrimination( $\delta 13 \mathrm{C}$ ) under severe water stress conditions (Table 2). $\delta 13 \mathrm{C}$ has been proposed as a selection criterion for improved drought tolerance in cereals (Condon et al. 2004). Carbon isotope discrimination, which strongly associates with transpiration efficiency, was used to select higher yield responses in sunflower and wheat under drought stress conditions (Richards 2006). Transpiration efficiency is defined as the ratio of biomass and water transpired, and transpiration efficiency is an important 
component of water-use efficiency (WUE) which can defined as the ratio of photosynthesis to transpiration ( $\mathrm{Xu}$ and Hsiao 2004). The establishment of physiological links between $\delta 13 \mathrm{C}$ and WUE has been useful in assessing genetic variation for water use efficiency (Obidiegwu et al. 2015). In view of this, understanding the inheritance of $\delta 13 \mathrm{C}$ could be useful for developing potato cultivars with high WUE (Anithakumari et al. 2012). However additional studies to understand transpiration efficiency, WUE and tuber yield under different levels of water stress are necessary.

In Chapter 4, some of the significant marker trait associations found with association mapping overlapped with QTLs detected for drought tolerance in a diploid potato population (Anithakumari et al. 2012; Chapter 3). The overlap between QTLs (other than those found on chromosome 5) on the same location in the bi-parental segregating populations and the diverse cultivar set is summarized in Table 2. On chromosome 4, a QTL for plant height was detected in the tetraploid cultivar set while a QTL for stem number was reported in the CXE diploid mapping population (Anithakumari et al. 2012). The QTL for stem number was reported under severe stress while the QTL for plant height was found under moderate stress. This indicates that the QTL locus on chromosome 4 affected two different traits under different stress severities. This presumably pleiotropic QTL region was detected in both tetraploid cultivars and diploid genotypes, but only under greenhouse conditions. This may suggest hat environmental differences between greenhouse and field influenced the expression of these traits. The environmental differences between field and greenhouse growing conditions include day length, radiation, temperature, relative humidity, soil type and growing space. Day length was short for the field experiment (Ethiopia) while it was longer for green house experiments (The Netherlands). Specific environmental differences between the green house experiment and field conditions where both experiments used similar CxE diploid potato genotypes and severe drought stress conditions has been discussed earlier in this discussion.

QTLs that co-located on chromosome 12 include shoot dry weight and root-stolon dry weight detected under mild drought stress with shoot fresh weight measured in vitro under well-watered condition (Table 2). QTLs for shoot dry weight and root-stolon dry weight explained 17 and $13 \%$ of phenotypic variation, respectively. Both QTLs were specific for 
drought stress conditions. Co-localization of shoot QTL with underground trait QTL can help in indirect selection of root and stolon trait since measuring underground traits is difficult and laborious. Shoot dry weight is also an important trait since it is linked with the whole canopy architecture, at least before the onset of senescence. Drought was shown to affect potato canopy architecture by decreasing leaf size and leaf expansion rate while limiting formation of new leaves and increasing the rate of senescence (Fleisher et al. 2008). Besides, higher shoot biomass production has been suggested to be linked with larger yield production under drought stress conditions (Schittenhelm et al. 2006). Shoot biomass measurements however are often destructive, and therefore not so easily included in selection trials. The canopy cover measurements and derived traits by modelling as described in Chapter 5 appear be a good, non-destructive, alternative for shoot biomass measurements, as discussed later in this Chapter.

The identification of drought tolerance traits that have good correlation with yield can be combined into a selection index to be used in a drought improvement breeding program. The construction of a selection index can be done by assigning a weighing scheme for each trait that has higher correlation with tuber yield under water stress conditions, assigning higher weight for the traits that contribute more to yield. In our experiment in Chapter 3, shoot traits had a good correlation with tuber yield and root traits and also explained a large amount of the overall phenotypic variance under water limited conditions. Other QTLs linked with tuberization (initiation of tuber formation) and stolonization (initiation of stolon formation) were also identified under water-limiting conditions (Chapter 5). Tuberization in potato is known to be regulated by the CDF-1 gene located on chromosome 5; however another QTL for tuberization was found on chromosome 9. The QTL detected on chromosome 9 for tuberization indicates that more factors in addition to the CDF-1 gene on chromosome 5 may influence the tuberization pathway under drought, and this QTL may be a starting point to elucidate how drought impacts the molecular mechanisms of the regulation of tuberization. This QTL also overlapped with QTL for chlorophyll content measured under water limiting conditions indicating a pleiotropic effect of this QTL. 
Table 2. Overview of overlapping QTLs identified under moderate (MS), severe drought stress (SS) and well-watered (WW) conditions using association mapping (AM) and biparental QTL mapping (BP mapping) under different environmental conditions.

\begin{tabular}{clccll}
\hline \multirow{2}{*}{ Chromosome } & Traits & Analysis & Treatment & Environment & References \\
\hline 4 & $\begin{array}{l}\text { Stem number } \\
\text { Plant height }\end{array}$ & BP mapping & SS & Greenhouse & Anithakumari et al. 2012 \\
& AM & GS & Greenhouse & \\
\hline 10 & $\delta^{13} \mathrm{C}$ & BP mapping & SS & Greenhouse & Anithakumari et al. 2012 \\
& Tuber yield & AM & WW & Greenhouse & Chapter 4 \\
\hline 12 & $\begin{array}{l}\text { Shoot fresh weight } \\
\text { Shoot dry weight } \\
\text { Root-stolon dry } \\
\text { weight }\end{array}$ & BP mapping & WW & In vitro & Anithakumari et al. 2011 \\
& AM & MS & Greenhouse & Chapter 4 \\
& & AM & MS & Greenhouse & Chapter 4 \\
\hline
\end{tabular}

\section{QTLs controlling developmental traits under short photoperiod}

Many studies were done in potato using single trait QTL analysis (Visker et al. 2003; Costanzo et al. 2005; Śliwka et al. 2008). However, the power of detecting QTLs linked to growth and developmental traits can be higher when employing multi-trait QTL analysis compared to analyzing traits separately. The power of multi-trait QTL analysis lies in its ability to detect closely linked chromosomal regions affecting several traits simultaneously (Jiang and Zeng 1995). The first QTL meta-analysis in potato was done by projecting individual QTLs discovered for late blight and maturity from several studies onto a consensus potato map where it was possible to identify co-localization of QTLs for the aforementioned traits (Danan et al. 2011). This approach allowed improvement of defining the genomic regions controlling the traits. Thus, in Chapter 2 we have used a multi-trait QTL analysis to dissect the complex genetic basis of potato development, grown under short day conditions. The CxE diploid mapping population was used and time series of developmental data including plant height, flowering and senescence along with agronomical traits were collected.

Growth and developmental of potato can be controlled by QTLs that have pleiotropic effects, and this has been shown in previous studies (Malosetti et al. 2006; Hurtado et al. 2012). The locus on chromosome 5 has been known to have pleiotropic effects. In Chapter 3 
and 4 we have also detected QTLs on chromosome 5 affecting growth and yield traits under mild as well as severe drought stress conditions. In the multi-trait QTL analysis we have detected several QTLs, other than the one located on chromosome 5, with pleiotropic effects controlling potato development as well as yield traits under short day conditions. From the proposed pleiotropic QTLs, a QTL present on chromosome 3 (C3) was shown be associated with faster growth (tall in height and few main stems) and lower number of tubers. Identifying such pleiotropic QTL allows making a link between agronomic and developmental traits. Such discovery has shown the power of multi-trait QTL analysis to dissect the genetic basis of physiological relationships of developmental traits for a better understanding of the complex developmental process in potato. In essence, multi-trait QTL analysis allowed us to detect QTLs with pleiotropic effects controlling above and below ground traits when compared with single trait analysis on chromosomes other than chromosome 5.

It has been reported that temperature and photoperiod are major environmental factors controlling development in potato (Ewing and Struik 1992). High temperature together with long day increases the life span of potato. This would mean that the onset or end of developmental traits such as flowering and senescence can be greatly influenced by temperature and photoperiod. Under long day conditions the onset of senescence is delayed compared to short day conditions (Hurtado et al. 2012). For example, Dutch potato cultivars used as reference in the CxE field experiments showed faster senescence under short days, indicating that maturation is accelerated under short days. The discovery of QTLs controlling developmental traits in potato under different photoperiod conditions can give a better insight when breeding for different environmental conditions. In our research, QTLs for the developmental traits (plant height, flowering and senescence) were detected on chromosome 1 and 2 in addition to 5 under short photoperiod, while under long day conditions (the Netherlands) QTLs for onset of senescence for CXE diploid potato were reported on chromosome 5, 7 and 9 (Celis-Gamboa 2002), indicating QTL by environment interaction. Furthermore QTLs for developmental traits (plant height, flowering and senescence) using the same CxE diploid potato genotypes have been reported under short and long day conditions where some of them were expressed across environments (e.g. on C3) while others were specific to a single environment (e.g. on C8) (Hurtado-Lopez 2012). 
This also suggests the presence of QTL by environment interactions. In our study, some of the QTLs identified for plant height, flowering and senescence were time dependent, being expressed at specific developmental stages while other QTLs were expressed for the whole developmental process. For instance QTLs identified for plant height on chromosome 2 were expressed for the whole growing process while QTLs detected on chromosome 1, 3, and 4 were expressed between onset and half-way of the growth process. This indicates that besides being influenced by environmental factors, expression of developmental QTLs is affected by the developmental stage of potato. The discovery of QTLs linked to the developmental process of potato under short day conditions along with the ones reported under long day (Celis-Gamboa 2002; Hurtado-Lopez 2012) has helped to uncover QTL by environment interaction, time dependent QTLs or QTLs with pleiotropic effect that increased our understanding of the complex genetic architecture of developmental traits in potato.

\section{Drought effect on canopy development}

The relationship between total yield and canopy cover of the potato crop can mainly be divided in three components; light interception by the crop canopy, conversion of intercepted light into dry matter, and partitioning of dry matter to tubers. Many studies have indicated that under non-stressed growing conditions there is strong linear relationship between tuber yield and canopy cover (Struik et al. 1990; Haverkort et al. 1991; Ospina 2016). In Chapter 5, we assessed the relationship between canopy cover and tuber yield production under drought-stressed conditions in a field trial. For this experiment selected CxE genotypes from the field drought stress experiment (conducted in Chapter 3 ) were used. We have used beta-thermal time estimation to describe the canopy developmental stages over the growing period and this approach allowed us to fit a canopy development curve and use parameters that define the curve shape. The use of thermal time in explaining the developmental progress of potato has been used by other researchers (Khan 2012; HurtadoLopez 2012; Ospina 2016). The biological relevance of the model-derived parameters was described in (Khan 2012) by assessing the dynamics of canopy cover and tuber bulking as a function of thermal time and Ospina et al. (2014) and Ospina (2016) have similarly evaluated the development of the canopy over time under contrasting (low and high) nitrogen levels using a diverse set of tetraploid cultivars and the diploid potato mapping population $\mathrm{SH} \times \mathrm{RH}$. In Chapter 5, beta thermal time estimations ( $\operatorname{tm} 1, \mathrm{t} 1, \mathrm{t} 2$, te), expressed 
in thermal days indicate the duration taken for the different developmental stages of canopy while AP1, AP2, AP3 express the area under the curve for the three developmental stages, reflecting the amount of intercepted radiation in these developmental stages.

It has been reported that drought stress can accelerate senescence (Levy et al. 2013). In line with these studies drought stress induced early onset of senescence (t2) in our experiments described in Chapter 5, with drought-stressed genotypes taking shorter time to complete their life cycle (te) then well-watered genotypes. Delaying senescence can increase the total amount of intercepted radiation and the photosynthetic capacity of the crop during its life cycle, which can positively affect yield. Maintaining aboveground biomass has been associated with better tuber yield production under drought stress conditions (Schittenhelm et al. 2006), and this is in line with studies that reported that there is a positive relationship between canopy coverage and tuber yield production under stress conditions (Jefferies and MacKerron 1987; Jefferies and Heilbronn 1991). We also found that there was a positive relationship between total area under the canopy curve (AUC) with tuber yield under stress conditions. However, the relationship was a bit less strong compared to well-watered conditions. We suggest further investigation of this relationship using larger numbers of genotypes. Nevertheless our results indicate that that canopy coverage can be used as a selection criterion for yield under both control and drought conditions. Canopy cover as measured by us has the additional advantage that it is a non-destructive measurement.

The effect of drought stress on canopy development starts with reduced leaf expansion (Levy et al. 2013) which contributes to yield loss (Jefferies and MacKerron 1987). In our study, reduction in canopy cover was reflected by reduced AUC under water stress conditions. This reduction was as a result of reduction of areas under the curve for each developmental stage (AP1, AP2, and AP3). Reduction in AP1 may suggest that there is limitation in rate of formation of new leaves as well as leaf expansion as this canopy developmental phase is characterized by the appearance of new leaves (Khan 2012). Reduction in AP2 may suggest that there is limited expansion of leaves as well as reduction in time that maximum canopy is maintained (low Vx and shorter t2-t1) while reduced AP3 may indicate accelerated senescence. Studies suggest that avoiding drought-induced premature senescence under water limited conditions is related with the crop's ability to 
sustain canopy expansion (Jefferies and MacKerron 1993). Maximum soil cover (Vx) attained was greatly reduced in response to drought stress. These parameters had a positive correlation with tuber yield under stress conditions suggesting their relevance to be considered as drought tolerance indicator. Maintaining canopy cover under drought stress is an indication of the crop's ability to maintain leaf water content (Chaves and Oliveira 2004; Blum 2011). As suggested by some studies (Chaves and Oliveira 2004; Blum 2011) higher leaf relative water content under water stress conditions promotes drought tolerance in plants and also helps in rapid recovery upon rehydration. The model-derived parameters calculated by us in Chapter 5 have shown to have biological relevance in explaining canopy development under drought stress conditions, thus these parameters can be used to indicate plant fitness under stress. We suggest that these parameters can be useful indicators for selection of better performing genotypes under water limited conditions in drought improvement programs. Furthermore, it can be interesting to look into the genetic components that might be underlying the model derived canopy parameters, which could help use canopy parameters in marker assisted selection.

\section{Potato breeding for drought tolerance}

Conventional breeding programs in potato have focused on selecting yield potential, tuber quality and resistance to diseases for many years. Breeding for tolerance to drought in potato is yet in its infancy. The complexity of drought tolerance breeding is further increased when simultaneously other biotic and abiotic stresses occur; the interactions between these stresses can make drought tolerance breeding even more challenging. However, the availability of genomic resources in potato such as the sequenced potato genome (The Potato Genome Sequencing Consortium 2011) will certainly help in improving drought tolerance of potato. This resource has helped in the identification of genes with a wide variety of functions and controlling many diverse traits including biotic stress resistance (Jupe et al. 2012; Jupe et al. 2013) and quality traits (Uitdewilligen et al. 2013; D'Hoop et al. 2014). Anchoring of QTLs identified for drought tolerance traits in our studies to the annotated potato genome sequence may provide target genes for marker assisted breeding and candidate gene approaches, as exemplified by the study of Muijen et al (2016), which combined phenotypic, genome and transcriptome data to find candidate genes for drought response and tolerance in drought. 
QTLs identified for drought tolerance traits have been used successfully through marker assisted breeding to improve yield under water-limited conditions in cereals such as rice (Steele et al. 2013), sorghum (Harris et al. 2007), maize (Ribaut and Ragot 2007) and barley (Tuberosa and Salvi 2006). Efforts in breeding for drought tolerance in potato have mainly been limited since drought was not considered as a major yield limiting factor and potato was not considered as a crop of major importance in drought-prone areas (Monneveux et al. 2013). However, in recent years drought stress has become an important abiotic stress for potato cultivation also because potato production is expanding in tropical areas (Obidiegwu et al. 2015). A complicating factor in drought tolerance breeding may be the suggestion that heritability for tuber yield is low under water stress conditions. (Cabello et al. 2014), as mentioned before. For drought tolerance traits to be used as a selection criterion, high heritability is one of the most important desirable traits that would help in predicting response to selection. However, in the current study (Chapter 3 and 4) drought tolerance traits had moderate to high heritability in line with other drought tolerance studies (Anithakumari et al. 2011; Anithakumari et al. 2012; Khan et al. 2015), indicating that this constraint may be less problematic than suggested.

For a comprehensive understanding of the genetic basis of drought tolerance, the tools of genomics offer the means to produce comprehensive data sets on changes in gene expression, protein profiles, and metabolites that result in response to water stress. Transcriptome analysis has provided a means for assessing genome-wide changes in gene expression in response to drought stress. In a genome-wide gene expression study using CxE diploid potato mapping population, (Anithakumari 2011) reported transcriptional variation in response to drought stress and chromosome 5 was reported to carry a hotspot for eQTL close to the maturity locus. Further analysis of eQTL using maturity locus as a covariate showed the eQTL hotspot to still be significant (Muijen et al. 2016), indicating that this locus that seems to be a major regulator of the drought response in potato is at least partly independent of maturity. Expression QTL analysis is a powerful approach for identification of genes underlying particular biological phenotypes (Chen et al. 2010). Many of the biological processes in plants including adaptive response to environmental changes are controlled by regulation of gene expression at the level of transcription. Gene expression 
differences under water limited conditions can give better insight about candidate genes involved in the regulation of adaptive responses. Further analysis on the construction of regulatory networks can help to select the best candidate gene that involves in protection or recovery from drought stress.

Recently developed genomic tools can give us more understanding of regulation pathways that are involved in drought responses, and such techniques included proteomics and metabolomics. The importance of metabolomics has long been acknowledged in plant abiotic responses (Quanbeck et al. 2012). Metabolomics has been used to characterize specific metabolic pathways involved in abiotic stresses (Broeckling et al. 2005). Information generated with metabolomics research can help establish a better understanding of the complex metabolomics network and their responses to environmental changes. Proteomics is also another powerful tool to analyze biochemical pathways and the complex response of plant to environmental stimuli. Proteomic studies helps to further understand the molecular mechanisms underlying responses to abiotic stress (Weckwerth and Kahl 2013) and it also provides a link between the transcriptome and metabolome (Gray and Heath 2005).

\section{Phenotyping for drought tolerance}

The importance of precise and accurate phenotyping in dissecting complex traits into genetic parameters has been emphasized (Tuberosa 2012). In order to unravel the genetic basis of complex traits, such as drought, genotypic information is associated with the corresponding phenotypic data. However, the development of genomics approaches has been very fast compared to the development of phenotypic technology in the past few decades. The success of marker assisted breeding depends on the successful exploitation of genetic variation as well as accurate phenotyping. Breeding experiments usually use large populations with many plants to be examined either in controlled (greenhouse) or open field environments, which makes phenotyping tedious and difficult. Recently, the development of high throughput precision phenotyping technology has made it possible to record morphological and physiological traits at higher frequency and more accurately. Precise phenotyping can help in reducing the gap between genotype and phenotype, enhance the capacity and speed of data collection and offers the possibility of detailed morphological and physiological measurements of plant characteristics. Desirable characteristics of precision 
phenotyping for drought experiments should include easy adaptation to the field conditions (reduced experimental error), ability to measure dynamic traits, such as canopy development and biomass accumulation. This may allow understanding the changes in the genetic architecture underlying a trait in response to drought stress. Desirable traits for drought tolerance should have higher heritability than yield itself and have a genetic correlation with yield. Moreover, sufficient genetic variability of traits in germplasm, and lack of yield penalties under favorable conditions are also considered as desirable features.

The first step in breeding potato for drought tolerance is to identify genetic variation for drought tolerance traits. This requires evaluating a set of genotypes that segregate for a number of traits. In our field drought stress experiment, we have used a diploid potato population that has been genetically characterized for drought and quality traits. One of the parents used in developing this population originates from a wild potato species, Solanum phureja, and wild potatoes are more likely to harbor alleles that can be used to improve potato for harsh conditions, such as drought. In this experiment, we have identified QTLS linked to above- and below-ground traits that had correlation with tuber yield under water stress conditions (Figure 1). These traits include plant height, shoot fresh and dry weight, root fresh and dry weight, and root length and the percentage of phenotypic variance explained were $33,52,54,37,45,30$, respectively. This suggests that tuber yield is determined by the aggregated effects of morphological traits. We suggest that these traits can be used as drought tolerance indicators. However, the ease of measurement for above and below ground trait varies; above ground traits are easier to measure. We observed high correlation of shoot traits with root traits in our experiments suggesting shoot traits can be used as indirect selection criteria for root traits. Furthermore, we suggest doing data collection over time so that we know which genomic regions are involved at the different developmental stages that may affect the end tuber yield production under prolonged severe drought stress. For example traits such as plant height, canopy coverage can be measured for different time points and identification of the genomics region controlling these traits can give more information of the dynamics of the different genes that may be active at the different phases of development. Furthermore, harvesting shoot biomass at different time points can also give more information on the genomic regions that influence biomass accumulation at the different phase of development. However, these mostly involve 
destructive measurements and may be difficult to execute for experiments involving very large sets of genotypes. Therefore, we suggest precision phenotyping technology which involves at least remote sensing and image analysis that allows capturing the dynamics of biomass accumulation and this may allow the analysis of genes regulated at the different developmental stages. Drone technology can also be used to produce aerial photography that allows assessing canopy cover. In Chapter 5, we performed canopy development assessment and studied its relationship with tuber yield production under water stress conditions. Parameters such as t2 can describe the effect on senescence and AUC as a measure for captured solar radiation showed a correlation with tuber yield under water stress and control conditions. We suggest further experiments with larger numbers of genotypes in order to look in more detail into the genomic regions that may control canopy development traits and through this, tuber yield. In the experiment of prolonged mild drought stress using diverse potato cultivars (Chapter 4), several genomic regions controlling plant height, shoot dry weight, root-stolon dry weight, stolonization and tuberization were discovered (Figure 1). In both mild and severe drought stress QTLs for plant height, shoot dry weight and tuberization were detected on chromosome 5. QTL detected for plant height on chromosome 4 was found to co-locate on the same location for QTL reported for stem number under severe stress conditions (Anithakumari et al. 2012). This suggests that there are some similar genomic regions involved in controlling drought tolerance traits under mild and severe water stress conditions. Further investigation of these QTL regions is needed in order to identify the genes involved. Further investigation of these QTLs for instance by linking the QTL effect to gene expression studies would help in identification of the determinant genes that can be used to improve potato for cultivation under water-limited conditions. 


\section{References}

Anithakumari A, Dolstra O, Vosman B, Visser RG, van der Linden CG (2011) In vitro screening and QTL analysis for drought tolerance in diploid potato. Euphytica 181 (3):357-369

Anithakumari AM (2011) Genetic dissection of drought tolerance in potato. PhD Thesis, Wageningen University, Wageningen,

Anithakumari AM, Nataraja KN, Visser RG, van der Linden CG (2012) Genetic dissection of drought tolerance and recovery potential by quantitative trait locus mapping of a diploid potato population. Molecular breeding : new strategies in plant improvement 30 (3):1413-1429. doi:10.1007/s11032-012-9728-5

Ashraf M (2010) Inducing drought tolerance in plants: recent advances. Biotechnology advances 28 (1):169-183. doi:10.1016/j.biotechadv.2009.11.005

Blum A (2011) Drought resistance-is it really a complex trait? Functional Plant Biology 38 (10):753-757

Broeckling CD, Huhman DV, Farag MA, Smith JT, May GD, Mendes P, Dixon RA, Sumner LW (2005) Metabolic profiling of Medicago truncatula cell cultures reveals the effects of biotic and abiotic elicitors on metabolism. Journal of experimental botany 56 (410):323336

Cabello R, Monneveux P, Bonierbale M, Khan MA (2014) Heritability of yield components under irrigated and drought conditions in Andigenum potatoes. American Journal of Potato Research 91 (5):492-499

Celis-Gamboa BC (2002) The life cycle of the potato (Solanum tuberosum L.): from crop physiology to genetics. PhD Thesis, Wageningen University, Wageningen. ,

Chaves M, Oliveira M (2004) Mechanisms underlying plant resilience to water deficits: prospects for water-saving agriculture. Journal of experimental botany 55 (407):23652384

Chaves MM, Maroco JP, Pereira JS (2003) Understanding plant responses to drought-from genes to the whole plant. Functional plant biology 30 (3):239-264

Chen X, Hackett CA, Niks RE, Hedley PE, Booth C, Druka A, Marcel TC, Vels A, Bayer M, Milne I (2010) An eQTL analysis of partial resistance to Puccinia hordei in barley. Plos One 5 (1):e8598

Condon AG, Richards R, Rebetzke G, Farquhar G (2004) Breeding for high water-use efficiency. Journal of experimental botany 55 (407):2447-2460

Costanzo S, Simko I, Christ BJ, Haynes KG (2005) QTL analysis of late blight resistance in a diploid potato family of Solanum phureja x S-stenotomum. Theoretical and Applied Genetics 111 (3):609-617. doi:DOI 10.1007/s00122-005-2053-1

Courtois B, Ahmadi N, Khowaja F, Price AH, Rami J-F, Frouin J, Hamelin C, Ruiz M (2009) Rice root genetic architecture: meta-analysis from a drought QTL database. Rice 2 (2-3):115128

D'Hoop BB, Keizer PLC, Paulo MJ, Visser RGF, van Eeuwijk FA, van Eck HJ (2014) Identification of agronomically important QTL in tetraploid potato cultivars using a marker-trait association analysis. Theoretical and Applied Genetics 127 (3):731-748. doi:10.1007/s00122-013-2254-y

D'hoop BB, Paulo MJ, Mank RA, van Eck HJ, van Eeuwijk FA (2008) Association mapping of quality traits in potato (Solanum tuberosum L.). Euphytica 161 (1-2):47-60. doi:DOI 10.1007/s10681-007-9565-5 
Danan S, Veyrieras J-B, Lefebvre V (2011) Construction of a potato consensus map and QTL meta-analysis offer new insights into the genetic architecture of late blight resistance and plant maturity traits. Bmc Plant Biol 11 (1):1

Deblonde P, Ledent J-F (2001) Effects of moderate drought conditions on green leaf number, stem height, leaf length and tuber yield of potato cultivars. European Journal of Agronomy 14 (1):31-41

Fleury D, Jefferies S, Kuchel H, Langridge P (2010) Genetic and genomic tools to improve drought tolerance in wheat. Journal of experimental botany 61 (12):3211-3222

Gebhardt C, Ballvora A, Walkemeier B, Oberhagemann P, Schuler K (2004) Assessing genetic potential in germplasm collections of crop plants by marker-trait association: a case study for potatoes with quantitative variation of resistance to late blight and maturity type. Mol Breeding 13 (1):93-102. doi:Doi 10.1023/B:Molb.0000012878.89855.Df

Godfray HCJ, Beddington JR, Crute IR, Haddad L, Lawrence D, Muir JF, Pretty J, Robinson S, Thomas SM, Toulmin C (2010) Food Security: The Challenge of Feeding 9 Billion People. Science 327 (5967):812-818. doi:10.1126/science.1185383

Godoy JA, Lunar R, Torres-Schumann S, Moreno J, Rodrigo RM, Pintor-Toro JA (1994) Expression, tissue distribution and subcellular localization of dehydrin TAS14 in saltstressed tomato plants. Plant Mol Biol 26 (6):1921-1934

Gray GR, Heath D (2005) A global reorganization of the metabolome in Arabidopsis during cold acclimation is revealed by metabolic fingerprinting. Physiologia plantarum 124 (2):236-248

Harris K, Subudhi P, Borrell A, Jordan D, Rosenow D, Nguyen H, Klein P, Klein R, Mullet J (2007) Sorghum stay-green QTL individually reduce post-flowering drought-induced leaf senescence. Journal of experimental botany 58 (2):327-338

Haverkort A, Uenk D, Veroude H, Van de Waart M (1991) Relationships between ground cover, intercepted solar radiation, leaf area index and infrared reflectance of potato crops. Potato Research 34 (1):113-121

Hurtado-Lopez PX (2012) Investigating genotype by environmnet interactions for developmental traits in potato. PhD Thesis, Wageningen Univeristy, Wageningen.,

Hurtado-Lopez PX, Tessema BB, Schnabel SK, Maliepaard C, Van der Linden CG, Eilers PHC, Jansen J, van Eeuwijk FA, Visser RGF (2015) Understanding the genetic basis of potato development using a multi-trait QTL analysis. Euphytica 204 (1):229-241. doi:10.1007/s10681-015-1431-2

Hurtado PX, Schnabel SK, Zaban A, Veteläinen M, Virtanen E, Eilers PH, Van Eeuwijk FA, Visser RG, Maliepaard C (2012) Dynamics of senescence-related QTLs in potato. Euphytica 183 (3):289-302

Iwama K (2008) Physiology of the Potato: New Insights into Root System and Repercussions for Crop Management. Potato Research 51 (3-4):333-353. doi:10.1007/s11540-0089120-3

Jefferies R, MacKerron D (1987) Aspects of the physiological basis of cultivar differences in yield of potato under droughted and irrigated conditions. Potato Research 30 (2):201217

Jefferies R, MacKerron D (1993) Responses of potato genotypes to drought. II. Leaf area index, growth and yield. Ann Appl Biol 122 (1):105-112

Jefferies RA, Heilbronn TD (1991) Water stress as a constraint on growth in the potato crop .1. Model Development. Agricultural and Forest Meteorology 53 (3):185-196. doi:10.1016/0168-1923(91)90056-v 
Jiang C, Zeng Z-B (1995) Multiple trait analysis of genetic mapping for quantitative trait loci. Genetics 140 (3):1111-1127

Jupe F, Pritchard L, Etherington GJ, MacKenzie K, Cock PJ, Wright F, Sharma SK, Bolser D, Bryan GJ, Jones JD (2012) Identification and localisation of the NB-LRR gene family within the potato genome. Bmc Genomics 13 (1):1

Jupe F, Witek K, Verweij W, Śliwka J, Pritchard L, Etherington GJ, Maclean D, Cock PJ, Leggett RM, Bryan GJ (2013) Resistance gene enrichment sequencing (RenSeq) enables reannotation of the NB-LRR gene family from sequenced plant genomes and rapid mapping of resistance loci in segregating populations. The Plant Journal 76 (3):530-544

Khan MA, Saravia D, Munive S, Lozano F, Farfan E, Eyzaguirre R, Bonierbale M (2015) Multiple QTLs linked to agro-morphological and physiological traits related to drought tolerance in potato. Plant Molecular Biology Reporter 33 (5):1286-1298

Khan MS (2012) Assessing genetic variation in growth and development of potato. PhD Thesis, Wageningen University, Wageningen

Kloosterman B, Abelenda JA, Gomez MDC, Oortwijn M, de Boer JM, Kowitwanich K, Horvath BM, van Eck HJ, Smaczniak C, Prat S, Visser RGF, Bachem CWB (2013) Naturally occurring allele diversity allows potato cultivation in northern latitudes. Nature 495 (7440):246250. doi:10.1038/nature11912

Kumar V, Singh A, Mithra SA, Krishnamurthy S, Parida SK, Jain S, Tiwari KK, Kumar P, Rao AR, Sharma $S$ (2015) Genome-wide association mapping of salinity tolerance in rice (Oryza sativa). DNA Research:dsu046

Lahlou O, Ledent J-F (2005) Root mass and depth, stolons and roots formed on stolons in four cultivars of potato under water stress. European Journal of Agronomy 22 (2):159173. doi:10.1016/j.eja.2004.02.004

Levy D, Coleman WK, Veilleux RE (2013) Adaptation of potato to water shortage: irrigation management and enhancement of tolerance to drought and salinity. American Journal of Potato Research 90 (2):186-206

Long NV, Dolstra O, Malosetti M, Kilian B, Graner A, Visser RG, van der Linden CG (2013) Association mapping of salt tolerance in barley (Hordeum vulgare L.). Theoretical and applied genetics 126 (9):2335-2351

Malosetti M, Visser R, Celis-Gamboa C, Van Eeuwijk F (2006) QTL methodology for response curves on the basis of non-linear mixed models, with an illustration to senescence in potato. Theoretical and applied genetics 113 (2):288-300

Monneveux P, Ramirez DA, Pino MT (2013) Drought tolerance in potato (S. tuberosum L.) Can we learn from drought tolerance research in cereals? Plant Science 205:76-86. doi:10.1016/j.plantsci.2013.01.011

Muijen D, Anithakumari A, Maliepaard C, Visser RG, Linden CG (2016) Systems genetics reveals key genetic elements of drought induced gene regulation in diploid potato. Plant, Cell \& Environment

Munoz-Mayor A, Pineda B, Garcia-Abellán JO, Antón T, Garcia-Sogo B, Sanchez-Bel P, Flores FB, Atarés A, Angosto T, Pintor-Toro JA (2012) Overexpression of dehydrin tas 14 gene improves the osmotic stress imposed by drought and salinity in tomato. Journal of plant physiology 169 (5):459-468

Obidiegwu JE, Bryan GJ, Jones HG, Prashar A (2015) Coping with drought: stress and adaptive responses in potato and perspectives for improvement. Front Plant Sci 6 (542). doi:10.3389/fpls.2015.0052 
Ospina C, van Bueren EL, Allefs J, Engel B, van der Putten P, van der Linden C, Struik P (2014) Diversity of crop development traits and nitrogen use efficiency among potato cultivars grown under contrasting nitrogen regimes. Euphytica 199 (1-2):13-29

Ospina CA (2016) Nitrogen use efficiency in potato: an integrated agronomic, physiological and genetic approach. PhD Thesis, Wageningen University, Wageningen,

Passioura JB (2002) Review: Environmental biology and crop improvement. Functional Plant Biology 29 (5):537-546

Prasanna BM, Araus JL, Crossa J, Cairns JE, Palacios N, Das B, Magorokosho C (2013) Highthroughput and precision phenotyping for cereal breeding programs. In: Cereal Genomics II. Springer, pp 341-374

Quanbeck SMM, Brachova L, Campbell AA, Guan X, Perera A, He K, Rhee SY, Bais P, Dickerson J, Dixon P (2012) Metabolomics as a hypothesis-generating functional genomics tool for the annotation of Arabidopsis thaliana genes of "unknown function". Frontiers in plant science 3:15

Ramírez D, Yactayo W, Gutiérrez R, Mares V, De Mendiburu F, Posadas A, Quiroz R (2014) Chlorophyll concentration in leaves is an indicator of potato tuber yield in watershortage conditions. Scientia Horticulturae 168:202-209

Ribaut J-M, Ragot M (2007) Marker-assisted selection to improve drought adaptation in maize: the backcross approach, perspectives, limitations, and alternatives. Journal of experimental botany 58 (2):351-360

Richards RA (2006) Physiological traits used in the breeding of new cultivars for water-scarce environments. Agricultural water management 80 (1):197-211

Robin S, Pathan M, Courtois B, Lafitte R, Carandang S, Lanceras S, Amante M, Nguyen H, Li Z (2003) Mapping osmotic adjustment in an advanced back-cross inbred population of rice. Theoretical and Applied Genetics 107 (7):1288-1296

Rolando JL, Ramírez DA, Yactayo W, Monneveux P, Quiroz R (2015) Leaf greenness as a drought tolerance related trait in potato (Solanum tuberosum L.). Environmental and Experimental Botany 110:27-35

Schittenhelm S, Sourell H, Löpmeier F-J (2006) Drought resistance of potato cultivars with contrasting canopy architecture. European Journal of Agronomy 24 (3):193-202. doi:10.1016/j.eja.2005.05.004

Simko I (2004) One potato, two potato: haplotype association mapping in autotetraploids. Trends in plant science 9 (9):441-448. doi:DOI 10.1016/j.tplants.2004.07.003

Śliwka J, Wasilewicz-Flis I, Jakuczun H, Gebhardt C (2008) Tagging quantitative trait loci for dormancy, tuber shape, regularity of tuber shape, eye depth and flesh colour in diploid potato originated from six Solanum species. Plant Breeding 127 (1):49-55

Steele K, Price A, Witcombe J, Shrestha R, Singh B, Gibbons J, Virk D (2013) QTLs associated with root traits increase yield in upland rice when transferred through marker-assisted selection. Theoretical and applied genetics 126 (1):101-108

Struik PC, Haverkort A, Vreugdenhil D, Bus C, Dankert R (1990) Manipulation of tuber-size distribution of a potato crop. Potato Research 33 (4):417-432

Teulat B, This D, Khairallah M, Borries C, Ragot C, Sourdille P, Leroy P, Monneveux P, Charrier A (1998) Several QTLs involved in osmotic-adjustment trait variation in barley (Hordeum vulgare L.). Theoretical and Applied Genetics 96 (5):688-698

Tourneux C, Devaux A, Camacho M, Mamani P, Ledent J-F (2003) Effects of water shortage on six potato genotypes in the highlands of Bolivia (I): morphological parameters, growth and yield. Agronomie 23 (2):169-179 
Tuberosa R (2012) Phenotyping for drought tolerance of crops in the genomics era. Frontiers in physiology 3:347. doi:10.3389/fphys.2012.00347

Tuberosa R, Salvi S (2006) Genomics-based approaches to improve drought tolerance of crops. Trends in plant science 11 (8):405-412. doi:10.1016/j.tplants.2006.06.003

Uitdewilligen J, Wolters AMA, D'Hoop BB, Borm TJA, Visser RGF, van Eck HJ (2013) A NextGeneration Sequencing Method for Genotyping-by-Sequencing of Highly Heterozygous Autotetraploid Potato. Plos One 8 (5). doi:10.1371/journal.pone.0062355

Visker M, Keizer L, Van Eck H, Jacobsen E, Colon L, Struik P (2003) Can the QTL for late blight resistance on potato chromosome 5 be attributed to foliage maturity type? Theoretical and Applied Genetics 106 (2):317-325

Vos J, Groenwold J (1989) Genetic differences in water-use efficiency, stomatal conductance and carbon isotope fractionation in potato. Potato Research 32 (2):113-121

Weckwerth W, Kahl G (2013) The handbook of plant metabolomics. John Wiley \& Sons,

Xu L-K, Hsiao TC (2004) Predicted versus measured photosynthetic water-use efficiency of crop stands under dynamically changing field environments. Journal of experimental botany 55 (407):2395-2411

Xue Y, Warburton ML, Sawkins M, Zhang X, Setter T, Xu Y, Grudloyma P, Gethi J, Ribaut J-M, Li W (2013) Genome-wide association analysis for nine agronomic traits in maize under well-watered and water-stressed conditions. Theoretical and applied genetics 126 (10):2587-2596

Yactayo W, Ramírez DA, Gutiérrez R, Mares V, Posadas A, Quiroz R (2013) Effect of partial root-zone drying irrigation timing on potato tuber yield and water use efficiency. Agricultural Water Management 123:65-70

Zhu CS, Gore M, Buckler ES, Yu JM (2008) Status and Prospects of Association Mapping in Plants. Plant Genome-Us 1 (1):5-20. doi:DOI 10.3835/plantgenome2008.02.0089 


\section{Summary}

Drought is a major threat to agricultural production, which makes drought tolerance a prime target for breeding approaches towards crop improvement. Drought is a complex polygenic trait and poses a challenge for drought tolerance breeding. Improving crops for drought tolerance at least requires the knowledge of the physiological mechanisms of the contributing traits and their genetic control. Thus, identification of genetic variation for drought tolerance is the first step towards drought tolerance breeding.

Potato is a crop ideally suited for cooler growing conditions and shortages of water from its optimum requirement can have significant effect on tuber yield production. To understand the genetic factors underlying drought tolerance in potato, we performed two years of extensive field drought stress experiments using the CxE diploid potato population that has been genetically well characterized. The genotypes were exposed to water limitation starting from tuber initiation, which progressed to severe drought stress. Morphological and physiological trait data were collected that allowed precise monitoring of the drought response of potato and this phenotypic data were used for QTL mapping. In addition, we examined potato cultivars for moderate drought tolerance under greenhouse conditions. Collected drought tolerance trait data for the cultivars was used for genome wide association mapping.

The drought tolerance evaluation and QTL analysis of the CXE genotypes under field conditions includes traits like shoot and root biomass (fresh and dry), yield and chlorophyll content. In total we identified 60 QTLs controlling those traits both under well-watered and drought stress conditions. In the drought tolerance evaluation of the potato cultivars under greenhouse conditions we identified significant marker trait associations for both above- and belowground traits. In both experiments, trait heritability ranged from moderate to high even under drought stress conditions. Many of the QTLs detected for drought tolerance traits were specific to either moderate or severe drought tolerance conditions. However, a few QTLs showed an overlap between these drought stress environments. This demonstrates the presence of common genomic regions controlling drought tolerance traits under moderate and severe drought stress conditions. 
From the two years of field drought stress experiments we selected a subset of genotypes that showed contrasting responses to drought stress. We used these genotypes to further examine the relationship between canopy development and tuber yield under drought stress conditions. Canopy development was measured for several time points and the data were used for curve fitting. From the fitted curve parameters related to the different developmental phase of canopy were extracted. We observed that the correlation between canopy parameters and tuber yield under drought stress conditions were less strong than well-watered conditions.

Understanding the complex developmental processes of potato requires proper characterization of plant morphology over time and identifying the genetic basis controlling these processes will lead to the better understanding of its genetic architecture. For this purpose, the CxE diploid potato genotypes were grown under well-watered field conditions and morphological traits were collected over several times along with agronomical data collected at end harvest. The data from the developmental traits that include plant height, flowering and senescence were used for curve fitting and parameters related to the different developmental stages were extracted. We used the agronomic traits together with plant development parameters in a multi-trait QTL analysis and several QTLs controlling these traits were identified. Some of the QTLs identified had a pleiotropic effect, demonstrating a genetic relationship between above and below ground traits of potato.

The evaluation of potato for drought tolerance under field and greenhouse conditions has resulted in the identification of several QTLs that can be interesting to be used for enhancing drought tolerance in potato. Furthermore, the use of model derived parameters gave a better insight into the relationship between canopy development and tuber yield under water stress conditions and we suggest that QTL mapping using these parameters for canopy development under stress conditions can lead to the identification of genomic regions controlling different aspects of canopy development and their role in tuber production. 




\section{Acknowledgements}

It is with great pleasure that I am writing this part of my thesis, since this thesis is the result of great help received from my heavenly Father and many peoples.

First of all, I would like to thank my heavenly Father, who has been my daily inspiration and courage in all the days of my study period. I am very grateful for You have been my strength. I would like to extend my acknowledgements to my promotor Richard Visser for accepting me as PhD candidate in the department of plant breeding. I would like to thank him for the invaluable inputs that I have received during our discussion times. I am very grateful for the important decisions made during the course of my PhD study.

I would like to thank my supervisor Gerard van der Linden for his support, inputs and valuable discussions that helped me complete my thesis. Thank you for all the pushes, it did bring out a stronger version of me both personally and scientifically.

I would like to thank Chris Maliepaard for his help in statistical analysis. Many thanks to Eric van de Weg for the great help I received in QTL mapping and thanks again for the valuable discussions we had.

I would like to extend my appreciations to Holetta agricultural research institute for the help I have received when carrying out my field experiment including experimental land provision and technical assistance. I would specifically like to thank Gbremedhin Gebremariam for providing an office space in my stay at Holetta. I would also like to thank Atsede Solomon, Abebe Chindi, and Melaku for sharing their knowledge about growing potatoes.

I would like to thank Melkassa agricultural research institute for allowing me to undertake drought stress experiments. I would like to extend my appreciations to Dr. Mohammod, Selamawit for making the administration process smooth. Many thanks to Derege, Birtukan and Mohamod for the soil analysis. Thank you Mezgebu for your data provision that helpd me to identify dry spell season of Melkassa. Many thanks to Mulugeta, Kassaye, Desta for facilitation of lab abd field works. I am highly indebted for Gezahegn for providing an office space during my stay at Melkassa. Thanks again Gezish for being a great friend. Thank you Dr. Kassahun for your inputs in my research work.

I would like to thank Berend-jan Domba and Kindu Gashu for their help in planting and phenotyping during the field experiment. Many thanks to Cesar for your help in canopy data analysis! 
Many thanks to plant breeding department secretaries; Anne, Nicole, letty and Janneke, for your help in handling my travels. Ernest and Dianka, thank you for being my paranymphs. My stay in Wageningen was made great with so many wonderfull people I have come to know, my apologies if I forgot to mention some names. Adisse and Geni, What a wonderful and joyous moments we shared together! I am grateful to have you in my life. Bethye, I enjoyed the time we spent together, Much Love!! Mahi, thank you for being a fun person to be with and it was always great to have a good laugh with you! Many thanks to Mehad, Kal, Elias, Mehadi, Ashenafi, Aderajew, and Mindaye for the very refreshing Amharic talk and laugh during lunch breaks! Thank you Baye for the good chats during coffee breaks. Rutty my dear, thank you for the great and insightful momemts we had together.

I am very grateful for the uplifting moments shared with the Christian fellowship groups at Wageningen. Many thanks to Amanuel Evangelical church for the great time I have, especially to Bereket, thank you for your willingness to always give your help. Amanuel, Thank you for the very enlighting discussion we had all the time.

My deepest acknowledgement goes to Jony, who have been my great friend and mentor, many blessings!

To my families, thank you so much for sharing my journey every step of the way. To my mom, thank you for always wanting the best thing for me, I am very grateful for your Love! To my sisters Hiwot, Fasika, and Wongu and brothers Zeki, Afiti, Dany thank you for always listening and encouraging me. Love you all! Many thanks to Aweke, Habtu, Koki, Yohannes, Yitayew, and Gech for your supports and encouragements.

Thank you

Biructawit B. Tessema

June 2017 


\section{About the author}

Biructawit Bekele Tessema was born on July 6, 1982 in Nazareth, Ethiopia. She obtained her BSc in Horticulture in 2003 from Jimma University College of Agriculture. She then joined Addis Ababa University for her Masters study in Applied Genetics and graduated in 2006. Her master's thesis was on molecular taxonomy of alpine plant, Swertia abyssinica species complex. Biructawit then worked as an assistant researcher at Addis Ababa University and University of Oslo, where she worked on phylogeography study of Afro-alpine plants. From October 2007, Biructawit worked as a lecturer in Jimma University College of Agriculture and Veterinary Medicine. In October 2009, she started her PhD study in plant breeding department, Wageningen University. Her PhD work on genetic studies towards elucidation of drought tolerance of potato is presented in this thesis. Since February 2016 Biructawit is working as a postdoctoral researcher at Center for Quantitative Genetics and Genomics, Aarhus University. She works on testing different breeding schemes and looking into the possibilities of implementing genomic selection for selfing and outcrossing plants. 

Writing or rewriting a project proposal

Title: Unraveling the genetic basis of drought tolerance in potato

Writing a review or book chapter

MSc courses

MSc courses
Modern statistics for the life sciences (ABG-30806)

Modern statistics for the life sciences (ABG-30806)
Breeding for Quality and Resistance (PBR-30306)

Laboratory use of isotopes

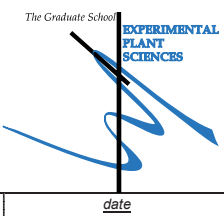

Mar 16, 2010

Mar 2010

Feb 22, 2010 Mar 01, 2010 13.5 credits $^{\star}$ Subtotal Start-up Phase

2) Scientific Exposure

EPS PhD student day, Wageningen University

Lunteren days and other National Platforms

Annuall Meeting 'Experimental Plant Sciences', Lunteren, NL
EPS PhD student day, Wageningen University

EPS theme symposia

EPS Theme 1 Symposium 'Developmental Biology of Plants', Wageningen University

EPS Theme 4 Symposium 'Genome biology', Radboud University, Nijmegen

EPS Theme 1 Symposium 'Developmental Biology of Plants', Leiden University

EPS Theme 3 Symposium 'Metabolism and adaptation', University of Amsterdam

Annuall Meeting 'Experimental Plant Sciences', Lunteren, NL

- Seminars (series), workshops and symposia

Symposium 'Photosynthesis: from femto to peta and from nano to Global'

Plant Breeding Annual Research day

Invited seminar by Prof. Kazuto Iwama, Hokkaido University (Japan), "Varietal difference in potato root Invited seminar by Prof. Nicole van Dam, Radboud University (Netherlands) :Multiple-stress Invited seminar by Andre Sugden, editor of Science: Writing for high impact joumals

Invited seminar by Gabino Sanchez Perez, Is your Research becoming Digital? Time to call the Invited semina by Paul Struik, Desigining drought-robust rice: scaling up genetic variation in Invited seminar by Gerard van der Linden, Growing rice like wheat: a multidisciplinary research program Seminar plus

Prof. Kazuto Iwama, Hokkaido University (Japan)

- International symposia and congresses

Ethiopian Horticultural Sciecnce Society, Jimma, Ethiopia

National workshop on potato, Bahir dar, Ethiopia

Wageningen 100 Years Congress 'Next Generation Plant Breeding', Ede, NL

Plant Genetics and Breeding Technologies, Vienna,Austria

5th European Plant Science Retreat (EPSR) for PhD students in 'Exprimental Plant Sciences', Ghent,

Presentations

Poster: EPS PhD day

Talk: National Workshop on Potato

Poster: 100 Years Plant Breeding

Poster: Plant Genetics and Breeding Technologies

Talk: 5th European Plant Science Retreat (EPSR)

Poster: Lunteren days

IAB interview

Meeting with a member of the International Advisory Board of EPS

- Excursions

Solagrow, Ethiopia

Solagrow, Ethiopia

Riik Zwan Breading Company, NL

May 20, 2011 Nov 30, 2012

Jan 28, 2010 Dec 07,2012 Jan 17,2013 Mar 22, 2013

Apr 22-23, 2013 Apr 14-15, 2014

Nov 05, 2009

Feb 08,2010

Feb 08, 2010

Feb 22. 2010

Dec 20, 2012

Feb 08, 2013

Mar 15, 2013

Jun 13, 2014

Jun 13, 2014

Feb 22, 2010

Feb 04- 05, 2011

Mar 12-14,2012

Nov $11-14,2012$

Feb $18-20,2013$

Feb 18-20, 2013

May 20, 2011

Mar 12-14,2012

Nov 11-14,2012

Feb 18-20, 2013

Jul 23-26, 2013

Apr 14-15, 2014

Nov 19, 2012

Jan 2012

Jan 11, 2013

Sep 27, 2013

\section{date}

Aug 26-28, 2013

2010, 2013-2014

2.9 credits $^{*}$

Litrature discussion group Plant breeding, Wagningen University

Individual research training

4) Personal development

Skill training courses
Working with Endnote

Working with Endnote

$\mathrm{PhD}$ competence assesment

Scientific Writing (6 days)

Techniques for Writing and Presenting a Scientific Paper (5 days)

Career Perspectives

Organisation of PhD students day, course or conference

Membership of Board, Committee or PhD council

\begin{tabular}{|c|c|}
\hline TOTAL NUMBER OF CREDIT POINTS & \\
\hline Herewith the Graduate School declares that the PhD candidate has complied with the educational & 38.1 \\
\hline
\end{tabular}

Herewith the Graduate School declares that the PhD candidate has complied with the educational
requirements set by the Educational Committee of EPS which comprises of a minimum total of 30 ECTS

* $A$ credit represents a normative study load of 28 hours of study. 



\section{Colophon}

The research presented in this thesis was funded by Nuffic-NPT project: Capacity Building for Sustainable Horticulture Development in Ethiopia (2007-2011), implemented by Wageningen UR, PTC+ and Jimma University College of Agriculture and Veterinary Medicine.

Cover design by Roos Marina Zaalberg

Printed by Digiforce | Proefschriftmaken.nl, De Limiet 26, 4131NC, Vianen, the Netherlands 